Functional analysis of psychiatric risk genes in zebrafish (Danio rerio): a focus on the L-type voltage-gated calcium channel subtypes $\mathrm{Ca}_{\vee} 1.2$ and $\mathrm{Ca}_{\mathrm{V}} 1.3$

By

Nancy Saana Banono

Thesis presented for the degree of

Philosophiae Doctor

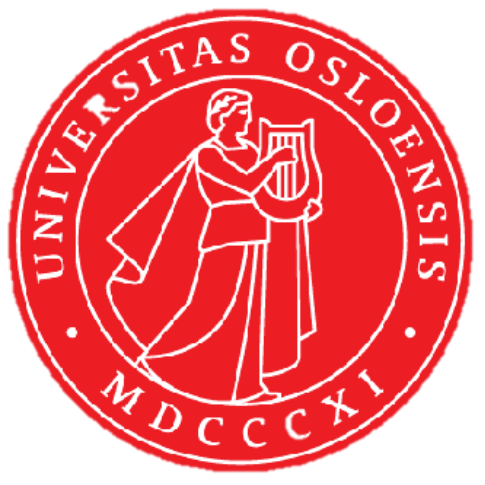

School of Pharmacy

Faculty of Mathematics and Natural Sciences

University of Oslo

2021 
(C) Nancy Saana Banono, 2021

Series of dissertations submitted to the

Faculty of Mathematics and Natural Sciences, University of Oslo No. 2434

ISSN $1501-7710$

All rights reserved. No part of this publication may be reproduced or transmitted, in any form or by any means, without permission.

Cover: Hanne Baadsgaard Utigard.

Print production: Reprosentralen, University of Oslo. 


\section{TABLE OF CONTENTS}

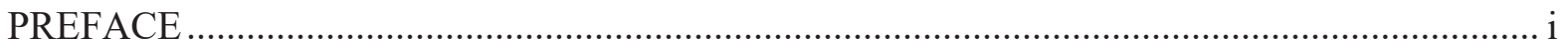

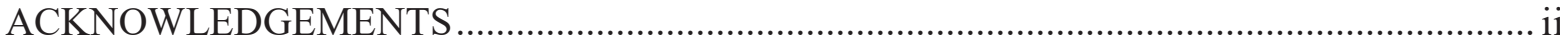

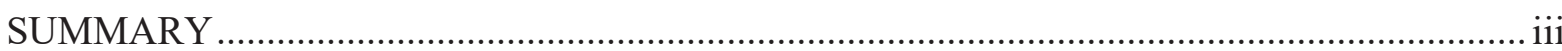

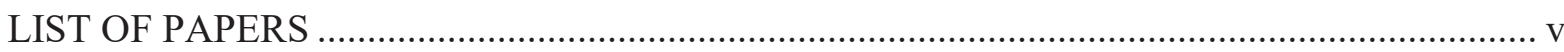

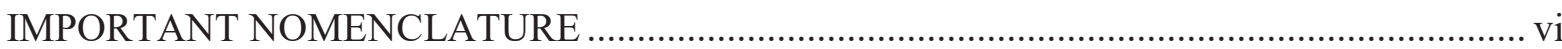

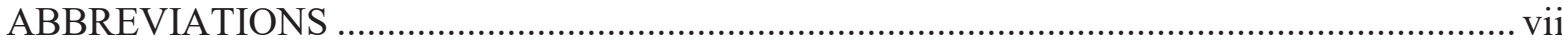

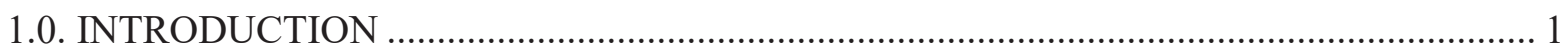

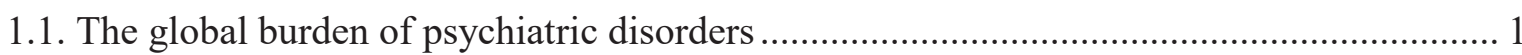

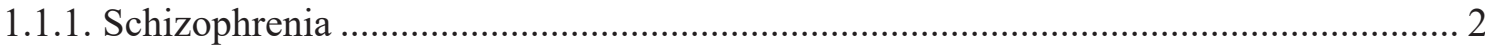

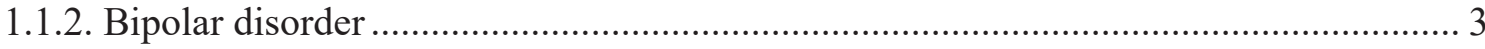

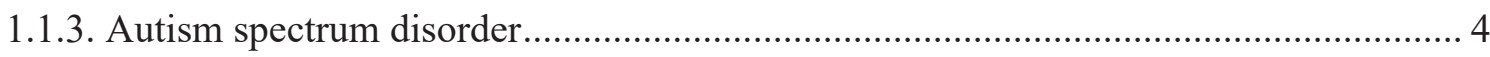

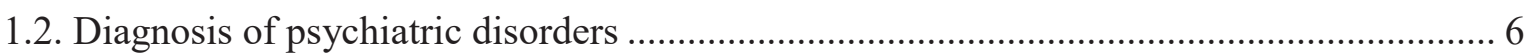

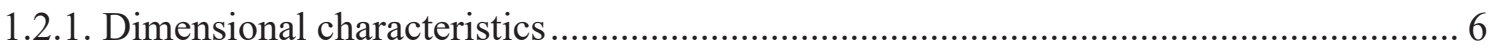

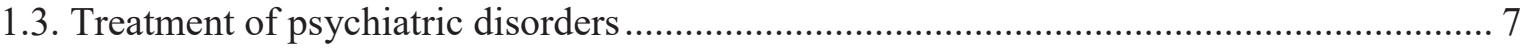

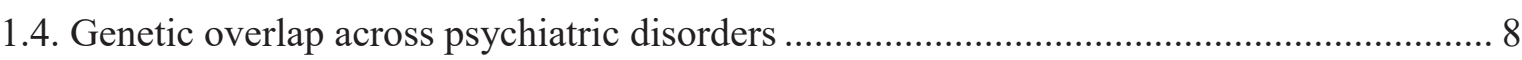

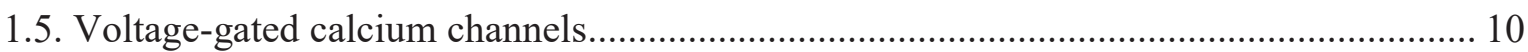

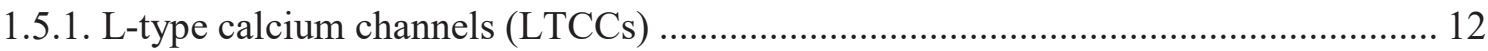

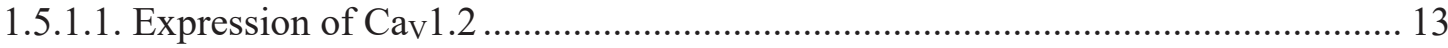

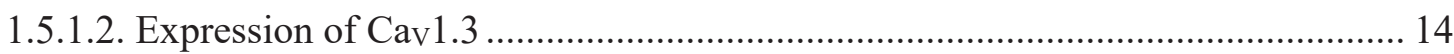

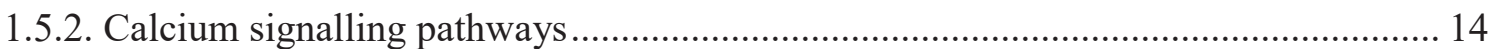

1.5.3. Association of L-type calcium channels with psychiatric disorders .......................... 16

1.5.3.1. Cav1.2 and psychiatric disorders ...................................................................... 17

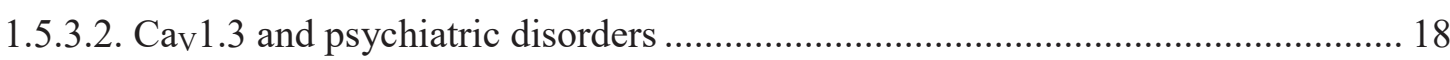

1.6. Phenotypic domains associated with LTCCs: Evidence from animal models................... 19 


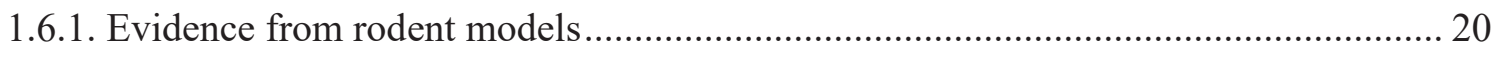

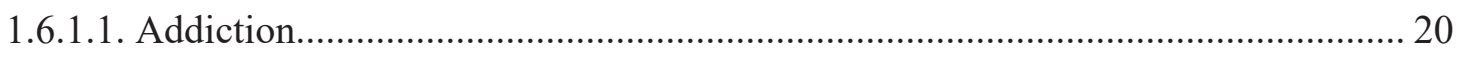

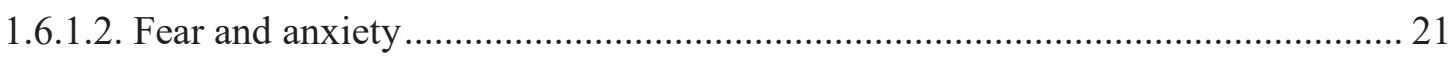

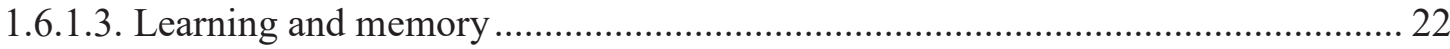

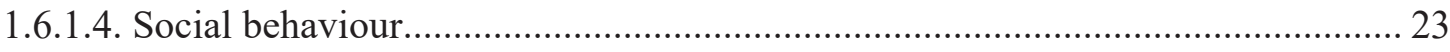

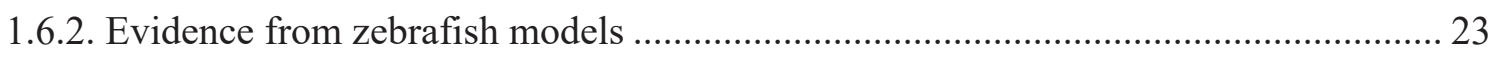

1.6.3. Relevant behavioural tests in zebrafish for modelling psychiatric phenotypes........... 24

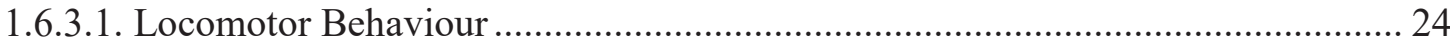

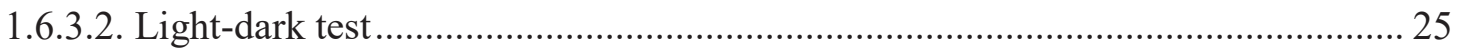

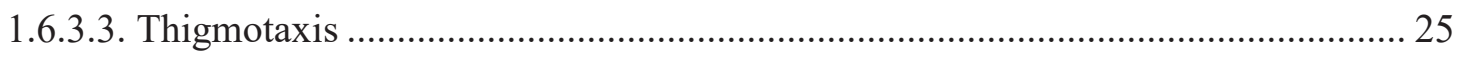

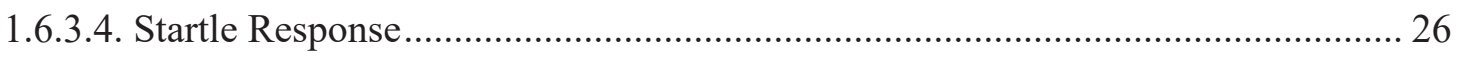

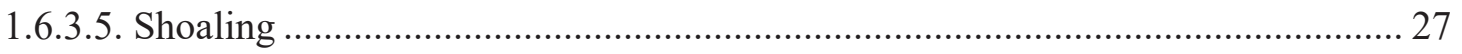

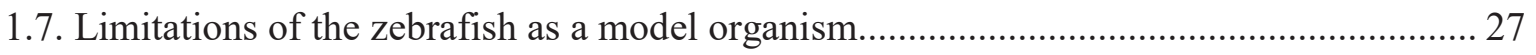

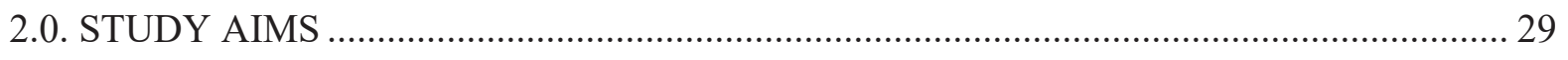

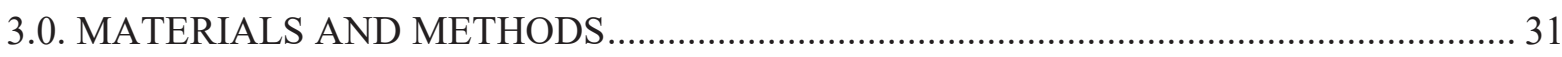

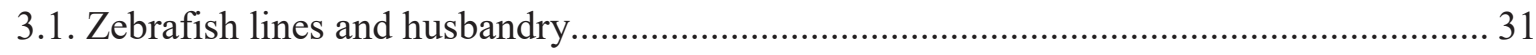

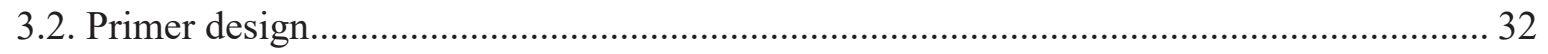

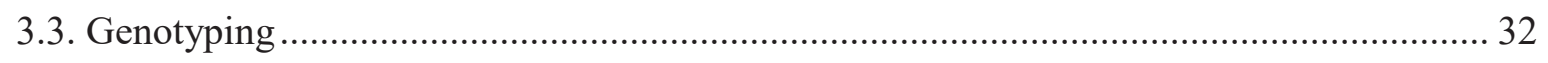

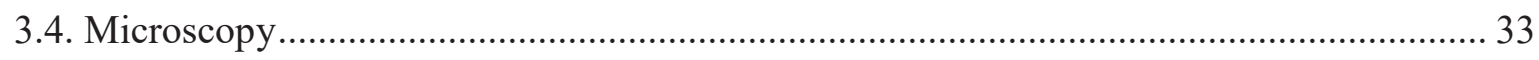

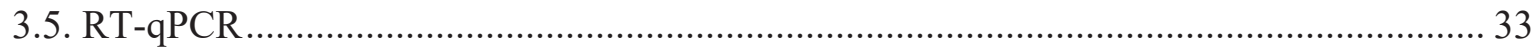

3.6. Preparation of samples for neurotransmitter measurement ............................................. 33

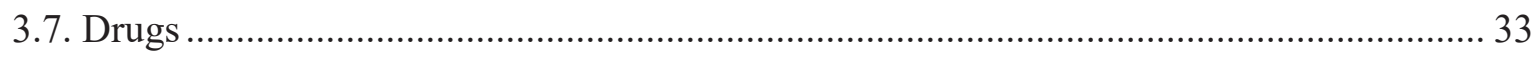

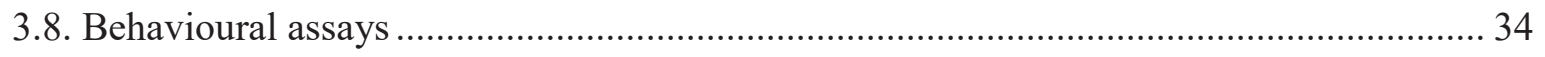

3.8.1. Prepulse inhibition of the acoustic startle response..................................................... 34

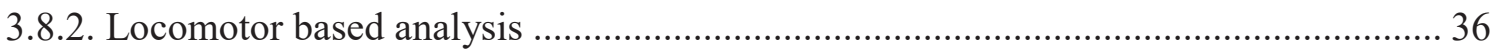




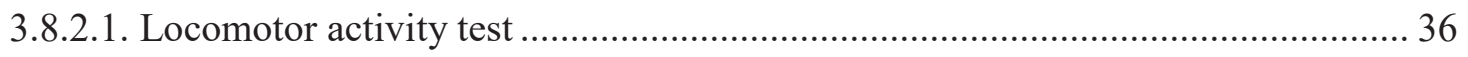

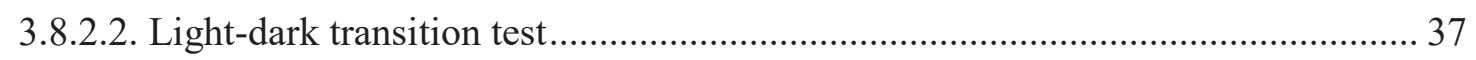

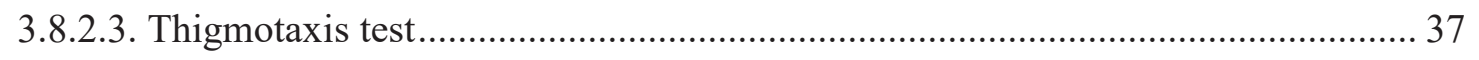

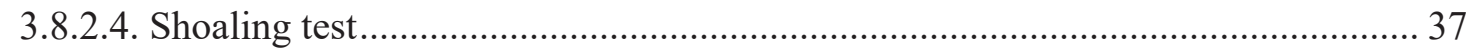

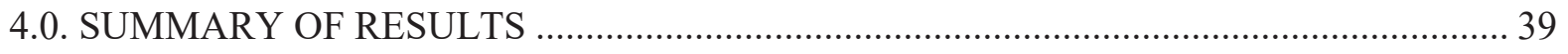

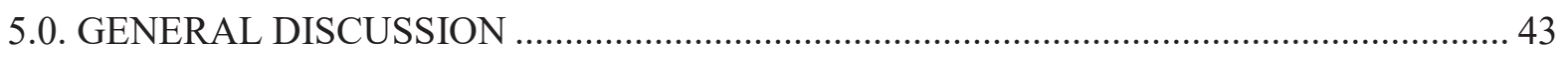

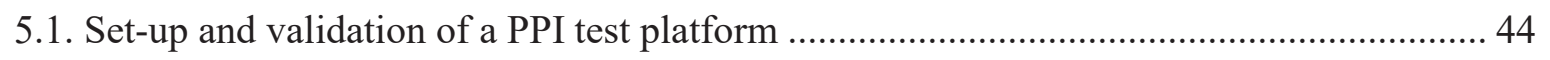

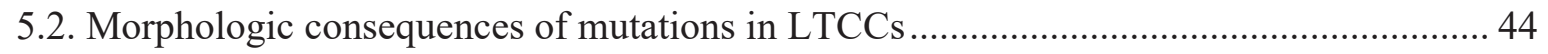

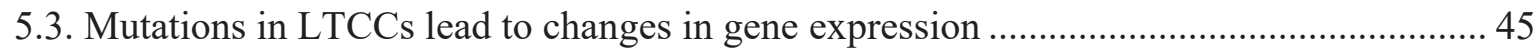

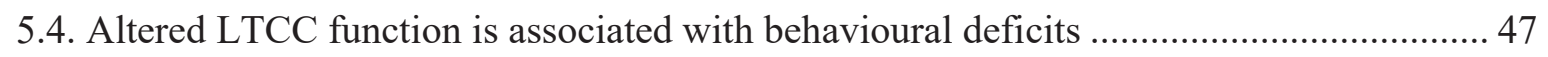

5.5. Altered LTCC function causes changes in neural oscillations and neurotransmitter levels

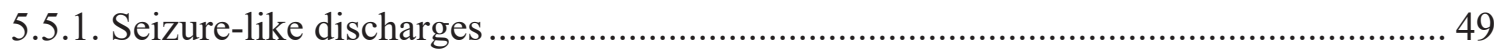

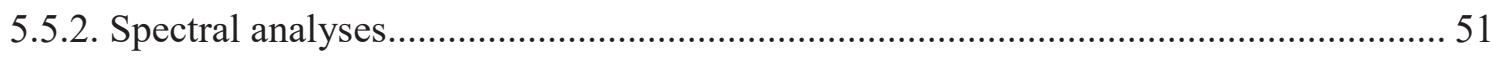

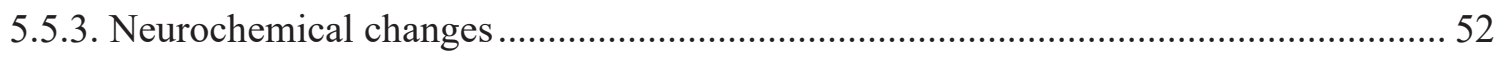

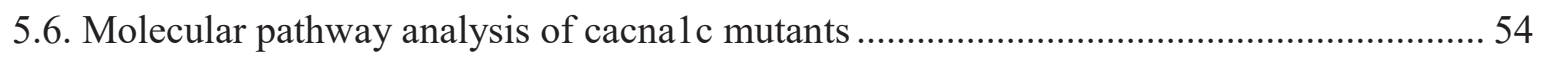

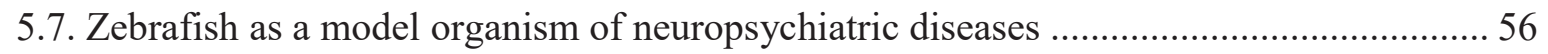

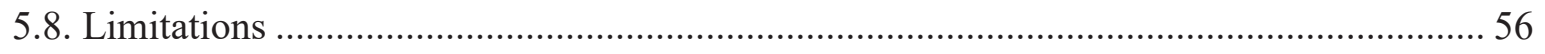

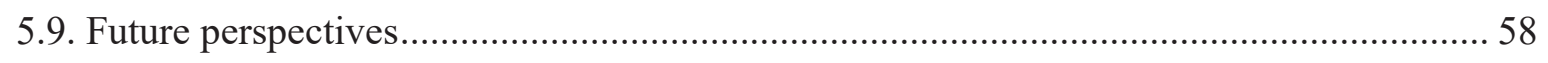

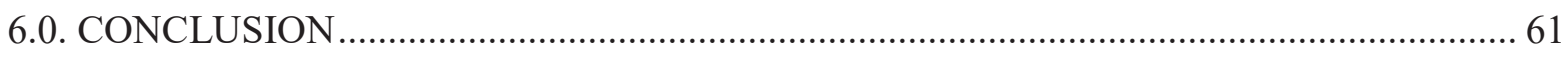

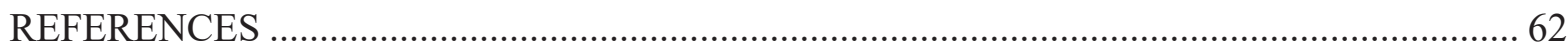

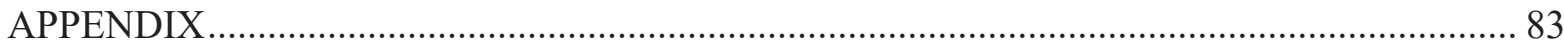

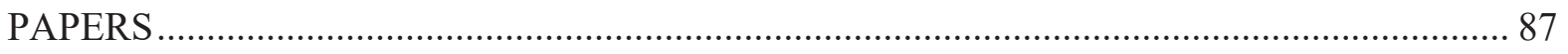





\section{PREFACE}

This thesis is submitted in partial fulfilment of the requirements for the degree of Philosophiae Doctor at the University of Oslo, Oslo, Norway. The work presented here was conducted at the Centre for Molecular Medicine Norway at the University of Oslo, under the supervision of Associate Prof. Camila V. Esguerra, with Prof. Ole A. Andreassen and Prof. Marianne Fyhn as cosupervisors. This work was supported by the Research Council of Norway, through the DigiBrain project (ISP, BIOTEK2021/DigiBrain). 


\section{ACKNOWLEDGEMENTS}

I wish to express my profound gratitude to Camila V. Esguerra, my principal supervisor for the confidence reposed in me right from when I first interviewed with you. Your mentorship and friendship have been valuable in keeping me through the changing scenes of my $\mathrm{PhD}$ training. To my co-supervisors, Ole A. Andreassen and Marianne Fyhn, I am truly grateful for the discussions, funding support, feedback on experiments and writing.

I am grateful to all members of the Esguerra lab whose smiles and friendship have kept me going amid the challenges. Wietske, Kinga, Karolina and to all co-authors, a very big thank you for helping me with various aspects of my experiments.

I wish to thank Ana Tavara, Alejandro P. Remiro, João Paulo R. P. Santana, Nelson T. Mathabela, Daniel Wroblewski and Rønnaug S. Kolve for the wonderful fish care - not forgetting all the weekend fish care staff.

A big thank you to my husband George for the love, friendship and encouragement. You have always kept things at home running while I navigated through the $\mathrm{PhD}$ journey. I believe you are worthy to be a co-owner of this thesis.

I appreciate you mum, dad and siblings, for all the support you have given right from the very beginning when I embarked on this journey.

To all friends and well-wishers, thank you for your diverse support including your words of encouragement. Of special mention are Sylvia and Svein Martin Asmussen, who have been my pseudo parents here in Norway - I appreciate the love.

Finally yet importantly, I wish to thank the entire DigiBrain team for discussions and feedback during our project meetings. 


\section{SUMMARY}

The zebrafish (Danio rerio) has emerged as a useful model to study neuropsychiatric diseases for several reasons. To set the tone for this thesis, it was important to understand the current state of the art within the field. In Paper I, we discussed the advantages of using zebrafish as a model of psychiatric disorders and the relevance of zebrafish larvae in schizophrenia studies. We further discussed the available pharmacological and genetic models of schizophrenia in zebrafish. Throughout, we present perspectives into some limitations and the way forward.

The advancement in genomic research has provided us with new opportunities to generate novel genetic models and harness them for drug discovery. With the advantages of the zebrafish already presented in Paper I, we sought to establish a behavioural test paradigm considered as an endophenotype, with the goal of characterising our genetic mutants and for use in the future to carry out a small-molecule compound library screen with the aim of identifying novel therapeutic candidates. Prepulse inhibition is a form of sensorimotor gating in which the startle response is attenuated when a weak non-startling stimulus is presented shortly before the startling stimulus. Deficits in prepulse inhibition are observed in schizophrenia patients and animal models while antipsychotics augment the prepulse inhibition response, making it a useful endophenotype for the genetic and pharmacological analysis of diseases such as schizophrenia. To facilitate the characterisation of the relevant genetic variants in the zebrafish model, we aimed to set-up a valid and robust screening assay. In Paper II, we combined the commercially available ZebraBox Revo system with the EthoVision software, to generate a protocol for the performance and analysis of the prepulse inhibition response in zebrafish larvae. Building upon previously published work, we were able to validate the protocol using three pharmacological modulators of the prepulse inhibition response i.e., apomorphine, haloperidol, and ketamine.

Genome wide association studies have identified several single nucleotide polymorphisms in the L-type voltage-gated calcium channel genes, $C A C N A 1 C$ and $C A C N A 1 D$, to confer greater risk for several psychiatric disorders. However, the mechanisms through which these genes cause disease are poorly understood. Thus, it has become increasingly important to unravel the role that these genes play in the development of disease. Non-human animal models such as the zebrafish provide 
a large opportunity to facilitate our understanding of the functional roles of the various psychiatric risk genes.

In Paper III, we used four locomotor-based assays and local field potential recordings to investigate the functional role of cacnalda in brain function using a cacnalda splice variant mutant. We showed that cacnalda haploinsufficiency in zebrafish leads to behavioural deficits reminiscent of schizophrenia. We also showed that the behavioural deficits could be improved or reversed using the antipsychotics haloperidol and risperidone and the mood stabiliser, valproate.

In Paper IV, using two zebrafish lines with different mutations in the cacnalc gene (one carrying a premature stop codon and the other, a splice variant mutation), we evaluated the effects of cacnalc on early brain function by assessing changes in gene expression, behaviour, local field potential activity recordings, neural oscillations and neurochemicals. We showed that cacnalc mutations lead to changes in gene expression of cacnalc and two downstream targets, which possibly explains the observed dysmorphologies, behavioural deficits, and gross brain excitability, as evidenced by spectral analysis of local field potential traces and imbalances in neurotransmitter levels.

Together, the results of this thesis show that mutations that alter the function of cacnalda and cacnalc in zebrafish lead to a wide range of phenotypes in larvae. The reported phenotypes are reminiscent of relevant functional domains observed in psychiatric disorders. 


\section{LIST OF PAPERS}

\section{Paper I}

Gawel, Kinga Aurelia; Banono, Nancy Saana; Michalak, Agnieszka \& Esguerra, Camila Vicencio (2019). A critical review of zebrafish schizophrenia models: Time for validation? Neuroscience and Biobehavioral Reviews. ISSN 0149-7634. 107, s 6- 22. doi: 10.1016 / j.neubiorev.2019.08.001

Paper II

Banono, Nancy Saana \& Esguerra, Camila V. (2020). Pharmacological validation of the prepulse inhibition of startle response in larval zebrafish using a commercial automated system and software. Journal of Visualized Experiments. ISSN 1940-087X. 161. doi: 10.3791/61423

Paper III

Banono, Nancy Saana; Gawel, Kinga Aurelia; De Witte, Linus \& Esguerra, Camila V. (2020). Zebrafish larvae carrying a splice variant mutation in cacnald: a new model for schizophrenia-like behaviours? Molecular Neurobiology. ISSN 0893-7648. doi: 10.1007/s12035-020-02160-5

\section{Paper IV}

Banono, Nancy Saana; Gawel, Kinga; Mäki-Marttunen, Tuomo; Kukula-Koch, van der Ent, Wietske; Wirginia; Fynn, Marianne; Einevoll, Gaute, T.; Andreassen, Ole A. \& Esguerra, Camila V. (2021). Functional characterisation of single nucleotide variants (SNVs) in the zebrafish neuropsychiatric risk gene cacnalc.

Manuscript in preparation 


\section{IMPORTANT NOMENCLATURE}

\begin{tabular}{|l|l|l|}
\hline Species & Gene & Protein \\
\hline Human & $\begin{array}{l}\text { Name in uppercase and } \\
\text { italicised }\end{array}$ & $\begin{array}{l}\text { Name in uppercase, not } \\
\text { italicised }\end{array}$ \\
\hline Mouse & $\begin{array}{l}\text { Only first letter in uppercase } \\
\text { anditalicised }\end{array}$ & $\begin{array}{l}\text { Name in uppercase, not } \\
\text { italicised }\end{array}$ \\
\hline Zebrafish & $\begin{array}{l}\text { Name in lowercase and } \\
\text { italicised }\end{array}$ & $\begin{array}{l}\text { Only first letter in uppercase, } \\
\text { not italicised }\end{array}$ \\
\hline
\end{tabular}

Reference: Genetic Nomenclature Guide, Trends in Genetics (1998). Retrieved from https://zfin.atlassian.net/wiki/spaces/general/pages/1818394635/ZFIN+Zebrafish+Nomenclature + Conventions 


\section{ABBREVIATIONS}

ADHD: attention deficit hyperactivity disorder

ASD: autism spectrum disorder

ASR: acoustic startle response

BDNF: Brain-derived neurotrophic factor

bp: base pair

BP: bipolar disorder

CACNA1C: voltage dependent L-type calcium channel, alpha $1 \mathrm{C}$ subunit

CACNA1D: voltage dependent L-type calcium channel, alpha 1D subunit

CaM: calmodulin

CaMKs: calcium/calmodulin-dependent protein kinases

Cav1.1 to 1.4: calcium voltage dependent channel, 1.1 to 1.4 representing the voltage of activation of the respective channel

CNS: central nervous system

CPP: conditioned place preference

CRE: cAMP response element

CREB: cyclic AMP response element binding protein

DNA: deoxyribonucleic acid

dpf: days post-fertilisation

DRD2: dopamine receptor 2

DSM: diagnostic criteria and statistical manual for mental disorders

E/I: excitation-inhibition

EEG: electroencephalography

Erk: extracellular signal-regulated kinases

fMRI: functional magnetic resonance imaging

GABA: gamma amino butyric acid

GOF: gain of function

GWAS: genome wide association studies

HALO: haloperidol

hiPSCs: human induced pluoripotent stem cells 
hpf: hours post fertilisation

hr: hour(s)

ICD: international classification of diseases

IID: interindividual distance

ISH: in situ hybridisation

LFP: local field potential

LOF: loss of function

LTCC: L-type calcium channels

LTP: long term potentiation

MAPK: mitogen activated protein kinase

M-cells: Mauthner cells

MDD: major depressive disorder

mg: milligram

min: minutes

ml: millilitres

mm: millimetres

mM: millimolar

ms: milliseconds

$\mathrm{mg} / \mathrm{ml}$ : milligram per millilitre

NAc: nucleus accumbens

NFAT: nuclear factor of activated $\mathrm{T}$ cells

NF-B: nuclear factor B

NMDA-R: N-methyl-D-aspartate receptor

NND: nearest neighbour distance

OCD: obsessive-compulsive disorder

PASNA: primary aldosteronism, seizures and neurological abnormalities

PCR: polymerase chain reaction

PD: psychiatric disorder

PET: positron emission tomography

PPI: prepulse inhibition

RCN: reticulospinal neurons 
RISP: risperidone

RNA: ribonucleic acid

RT-qPCR: reverse transcriptase quantitative polymerase chain reaction

SCZ: schizophrenia

sec: second(s)

SNP: single nucleotide polymorphism

TMD: transmembrane domain

TS: Timothy syndrome

VGCC: voltage-gated calcium channels

VPA: valproic acid

VTA: ventral tegmental area

v/v: volume by volume

WISH: whole mount in situ hybridisation

WT: wild type

YLD: years lived with disability

$\mu \mathrm{M}$ : micromolar

5HT-2R: serontonin receptor 2 


\subsection{INTRODUCTION}

\subsection{The global burden of psychiatric disorders}

Psychiatric disorders (PDs) such as schizophrenia (SCZ), bipolar disorder (BP), depression, anxiety and autism spectrum disorder (ASD) contribute significantly to the global burden of diseases $-7 \%$ and $19 \%$ to disability adjusted life years and years lived with disability respectively (Rehm and Shield, 2019). These disorders are largely chronically disabling, thus not just affecting the individual's quality of life, but also that of their families and society in general. PDs contribute to mortality, interact with other health conditions, and act as a cause or consequence of injury among others, altogether contributing significantly to the global burden of disease (Prince et al., 2007).

The aetiology of psychiatric diseases arises from a complex interplay of genetic, environmental and social factors (Uher and Zwicker, 2017). Environmental and social factors such as in utero and perinatal conditions, childhood trauma, urban residency, social isolation, discrimination, poverty and substance use is associated with increased risk for PDs (Uher and Zwicker, 2017). Epidemiological studies have shown that PDs tend to run in families, giving credence to a genetic underpinning of the diseases (Uher and Zwicker, 2017). Twin and adoption studies for instance, have estimated a heritability of more than $80 \%$ for SCZ, ASD and BP (Burmeister, McInnis and Zöllner, 2008; Winchester, Pratt and Morris, 2014). PDs are highly polygenic meaning that they are not caused by single mutations but a complex interplay of numerous pathogenic gene variants (Uher and Zwicker, 2017; Sullivan and Geschwind, 2019). Over the years, enough evidence has been provided to the effect that the genetics of PDs do not follow a simple Mendelian pattern of inheritance but rather, result from multiple gene variants that increases an individual's risk to a certain extent but is not sufficient on its own to cause disease. Thus, each variant contributes to the overall disease in unique small ways and non-genetic factors play crucial roles in the overall manifestation of the disease (Cross-Disorder Group of the Psychiatric Genomics Consortium, 2013; Uher and Zwicker, 2017). 


\subsubsection{Schizophrenia}

$\mathrm{SCZ}$ affects about $1 \%$ of the global population, and is characterised by psychotic symptoms (e.g., hallucinations or delusions), leading to loss of contact with reality (positive symptoms). In addition, patients often have impaired motivation, reduction in spontaneous speech, and social withdrawal (negative symptoms), in addition to cognitive dysfunction (Patel et al., 2014), see table 1. Symptom onset mostly starts in late adolescence to early adulthood, while cognitive deficits are often present at the pre-morbid phase (Fish et al., 1992; Mäki et al., 2005; Insel, 2010b; Patel et al., 2014). The negative and cognitive symptoms are generally chronic while the positive symptoms are associated with relapse and remission (Owen, Sawa and Mortensen, 2016).

Each category of symptoms tends to be influenced by unique or overlapping brain regions and circuitry. The positive symptoms for instance tend to be associated with dysregulation of the dopaminergic system (dopamine hyperfunction) although this does not explain the full spectrum of symptoms, while dysregulation in glutamatergic function (overall glutamate hypofunction) is associated with cognitive and negative symptoms. Dysfunction of parvalbumin-positive interneurons (inhibitory neurons) in the cerebral cortex and hippocampus is one of the key theories behind the observed glutamatergic dysfunction observed in SCZ (Owen, Sawa and Mortensen, 2016). In addition, the neuropeptide systems (corticotropin-releasing factor, orexin and neuropeptide Y) appear to contribute to cognitive symptoms. The monoamine oxidases play a role in the negative symptoms while cholecystokinin is associated with positive symptoms (Rich and Caldwell, 2015). Other hypotheses regarding the pathophysiology of SCZ involve excitationinhibition (E/I) imbalances, deficient myelination and altered functional connectivity (Insel, 2010b; Dawson, Morris and Pratt, 2015). Specifically, network and pathway analysis implicate dysregulation in calcium signalling, synaptic and immune function as well as epigenetic changes such as histone methylation in SCZ (Smoller et al., 2019).

Antipsychotics are the main class of drugs used in the treatment of SCZ. These drugs are categorised into two main groups - first- and second-generation antipsychotics. The firstgeneration antipsychotics were identified in the 1950's serendipitously and function by blocking dopamine receptor 2 (DRD2) to a high degree with low serotonin receptor 2 (5HT-2R) blockage. On the other hand, the second-generation antipsychotics were identified more recently in the 
1990's where they bind and affect not only DRD2 but also other neurotransmitter receptors, such as serotonin receptors 2 (5HT-2R) (Patel et al., 2014; Owen, Sawa and Mortensen, 2016).

\subsubsection{Bipolar disorder}

BP is a chronic mood disorder presented as episodes of mania and depression with normal mood in between. Impulsivity, psychosis and high-risk behaviours often accompany the episodes of mania (Vieta et al., 2018). BP has different sub-categories with the common ones being Bipolar I and II. Bipolar II is the most common, with patients suffering from other psychiatric comorbidities and raised frequency of suicidal thoughts, which often lead to death. In Bipolar I disorder, patients must present at least one episode of mania (most patients present MDD as well, although MDD is not required for diagnosis) while in Bipolar II, patients must present at least one hypomanic episode and one MDD episode see table 1. In fact, Bipolar II is considered the most common presentation of the illness (Grande et al., 2016; Vieta et al., 2018).

Imbalances in monoaminergic (serotonergic, noradrenergic and dopaminergic) neurotransmitter systems is thought to contribute to the development of BP. Aside from the "hypothalamicpituitary-thyroid" and the "hypothalamic-pituitary-adrenal" axes hypothesis of BP pathophysiology, signalling pathways involved in dendritic sprouting and neural plasticity such as brain-derived neurotrophic factor (BDNF), calcium signalling, inflammatory cytokines among others, seem to play vital roles in the regulation of mood and cognitive functions (Sigitova et al., 2017; Vieta et al., 2018). Indeed, analysis of post-mortem brain tissue of BP patients reveal loss of dendritic spines (Konopaske et al., 2014). Because of the numerous uncertainties regarding the mechanisms underlying BP, the race to identify relevant pathways and brain circuitry continues (Vieta et al., 2018).

Lithium was the first drug approved for the treatment of mania in BP. Ever since, ten drugs have been approved for use in adults, whereas in children, only five drugs are currently used. Development of adjunctive therapy, such as electroconvulsive therapy among others, are also being advanced (Vieta et al., 2018). 


\subsubsection{Autism spectrum disorder}

ASD is a neurodevelopmental disorder often present within the first two years of life, although it can be diagnosed at later stages of life. The disorder encompasses a range of symptoms that involve challenges with verbal and non-verbal communication, social skills and repetitive behaviours (Lord et al., 2020). ASD is a spectrum with the type and severity of clinical characteristics being highly variable see table 1 .

ASD is often associated with psychiatric, neurodevelopmental and physical comorbidities, some of which are not part of the diagnostic criteria, but negatively impact the wellbeing of patients. The psychiatric comorbidities include - attention deficit hyperactivity disorder (ADHD, 28.2\% prevalence), irritability and aggression (25\% prevalence), various forms of anxiety (social anxiety, generalised anxiety, separation anxiety in younger children, and phobias) and depression. The form of irritability and aggression may vary in young children vis-à-vis their adult counterparts. It is easier to observe anxiety and depression in ASD individuals who are verbally fluent. On the other hand, epilepsy, intellectual and academic learning disabilities are examples of the coexisting neurodevelopmental deficits whereas gastrointestinal and sleeping problems are some of the common physical conditions that coexist with ASD (Lord et al., 2018).

ASD is viewed as a condition caused by changes in brain organisation during early development. Indeed, neuroimaging studies have revealed differences in brain volume and connectivity (Lord et $a l ., 2018)$. For instance, one of the theories underlying the brain volume differences has to do with abnormal neuronal migration, which lead to a myriad of phenotypes such as thickening of the frontal lobe and macrocephaly (Yenkoyan et al., 2017). Moreover, progress in genetic studies suggest that alterations in synaptic development and function, transcriptional regulation, WNT signalling, chromatin remodeling, and mitogen-activated protein kinase (MAPK) signalling may be important in the pathophysiology of ASD (Vorstman et al., 2017). Furthermore, other factors such as impaired mitochondrial energy metabolism and immune activity, E/I imbalances, gastrointestinal abnormalities, toxins, infections among others have been suggested to cause ASD and their emerging hypotheses are being investigated (Yenkoyan et al., 2017).

Pharmacological based treatments are used in the management of coexisting conditions but not ASD itself. For managing ASD proper, behavioural and social mediated interventions that involve both parents and patients are used (Lord et al., 2018). 
Table 1. Clinical features of selected psychiatric disorders. ADHD = attention deficit hyperactivity disorder. Adapted from Owen et al, (2016).

\begin{tabular}{|c|c|c|c|c|}
\hline & Schizophrenia & Bipolar disorder & Autism & ADHD \\
\hline $\begin{array}{l}\text { Core } \\
\text { features }\end{array}$ & $\begin{array}{l}\text { Delusions, } \\
\text { hallucinations, } \\
\text { impaired } \\
\text { motivation, } \\
\text { reduction in } \\
\text { spontaneous } \\
\text { speech, and social } \\
\text { withdrawal }\end{array}$ & $\begin{array}{l}\text { Episodes of } \\
\text { elated mood and } \\
\text { episodes of } \\
\text { depressive mood }\end{array}$ & $\begin{array}{l}\text { Social and } \\
\text { communication } \\
\text { difficulties, and } \\
\text { restricted } \\
\text { repetitive } \\
\text { behaviours }\end{array}$ & $\begin{array}{l}\text { Inattention, } \\
\text { hyperactivity, } \\
\text { and } \\
\text { impulsivity }\end{array}$ \\
\hline $\begin{array}{l}\text { Additional } \\
\text { features }\end{array}$ & $\begin{array}{l}\text { Cognitive } \\
\text { impairment, } \\
\text { episodes of elated } \\
\text { mood, and } \\
\text { episodes of } \\
\text { depressive mood }\end{array}$ & $\begin{array}{l}\text { Delusions and } \\
\text { hallucinations }\end{array}$ & $\begin{array}{l}\text { Cognitive } \\
\text { impairment, } \\
\text { delusions, and } \\
\text { hallucinations }\end{array}$ & $\begin{array}{l}\text { Cognitive } \\
\text { impairment }\end{array}$ \\
\hline $\begin{array}{l}\text { Heritability } \\
\text { estimate }\end{array}$ & Roughly $80 \%$ & Roughly $85 \%$ & Roughly 58\% & Roughly $75 \%$ \\
\hline $\begin{array}{l}\text { Average age } \\
\text { of onset }\end{array}$ & $16-30$ years & $18-40$ years & $<3$ years & $7-12$ years \\
\hline $\begin{array}{l}\text { Drug } \\
\text { treatment }\end{array}$ & Antipsychotics & $\begin{array}{l}\text { Antidepressants } \\
\text { and mood } \\
\text { stabilisers }\end{array}$ & $\begin{array}{l}\text { No recommended } \\
\text { drug treatment; } \\
\text { treatment for } \\
\text { comorbidities if } \\
\text { present }\end{array}$ & Stimulants \\
\hline
\end{tabular}




\subsection{Diagnosis of psychiatric disorders}

Currently, there are two widely established systems for the classification of PDs - chapter V of the tenth international classification of diseases (ICD-10) and the diagnostic criteria and statistical manual for mental disorders (DSM-5) (Cuthbert and Insel, 2013; Smoller, 2013). The ICD-10 and DSM-5 rely on interviewing and examining the mental status of patients and classify them into descriptive diagnostic categories (Insel, 2010a; Cuthbert and Insel, 2013; Smoller, 2013). For instance, the interviews/examinations reveal whether patients have mood symptoms or psychosis (hallucinations, delusions), as well as behaviour and personality disturbances. Further testing can provide intelligence quotient and cognitive impairment assessments (Smith, 2008; Cuthbert and Insel, 2013). Due to the phenotypic similarity and heterogeneity of several PDs, diagnosing can be challenging (Burmeister, McInnis and Zöllner, 2008; Cuthbert and Insel, 2013). There are reports suggesting that most diagnoses are reliable when the categorical diagnosis tools are used, while the validity (relationship to disease mechanisms) is low (Burmeister, McInnis and Zöllner, 2008; Cuthbert and Insel, 2013; Regier et al., 2013).

\subsubsection{Dimensional characteristics}

Recently, the "Research Domain Criteria" which categorises symptoms into dimensions across diagnostic categories is being championed by the National Institutes of Health (Cuthbert and Insel, 2013; Krystal and State, 2014). The use of electrical and neuroimaging modalities (such as electroencephalography (EEG) and neuroimaging techniques (fMRI, PET, etc.)) and laboratory tests (serum, urine and cerebrospinal fluid tests) are considered "important adjuncts" for supporters of a combinatorial approach (Smith, 2008). Whereas the electric and neuroimaging modalities help to diagnose the neurological basis of psychiatric symptoms (Insel, 2009; Krystal and State, 2014), laboratory tests are helpful for diagnosing the underlying medical cause, i.e., are the symptoms due to genetic alterations and may be used to monitor the levels of "psychotropic medications" possibly predicting potential side effects (Smith, 2008). Together, these facilitate the "monitoring and progression" of disease (Smith, 2008; Krystal and State, 2014).

There is a growing use of spectral analysis of EEG datasets with the aim of finding signatures (i.e., brain oscillations) relevant to particular PDs. Brain oscillations are rhythmic and sometimes 
repetitive electrical activity generated in the neural tissue either spontaneously and/or in response to stimuli (Uhlhaas et al., 2008; Uhlhaas and Singer, 2010). Brain oscillations are divided into 5 sub frequency bands namely delta $(1-4 \mathrm{~Hz})$, theta $(4-8 \mathrm{~Hz})$, alpha $(8-13 \mathrm{~Hz})$, beta $(13-30$ $\mathrm{Hz})$, and gamma $(30-200 \mathrm{~Hz})$ (Uhlhaas et al., 2008). Changes in brain oscillations have been reported in various psychiatric and neurological disorders (Uhlhaas and Singer, 2010; Kam et al., 2013). Indeed, modified power spectra of delta (0.5-5 Hz) (Sponheim et al., 1994; Duan et al., 2015), alpha-theta (4-13 Hz) (Sponheim et al., 1994) and beta-gamma (25-200 Hz) (Uhlhaas and Singer, 2013) oscillations are thought to be neurophysiological biomarkers of SCZ. Lowfrequency (delta/theta) oscillations in the thalamocortical system are elevated in schizophrenia during wakefulness and are also induced in the $N$-methyl-D-aspartate receptor (NMDA-R) hypofunction rat model (Duan et al., 2015). Several neurotransmitters have been shown to influence oscillations - gamma-amino butyric acid (GABA), glutamate and acetylcholine are the major influencers of theta and gamma oscillations whereas glutamate, acetylcholine and serotonin influence alpha oscillations. In addition, beta oscillations are generated by the interplay of glutamate, GABA and dopamine (Uhlhaas et al., 2008; Moran and Hong, 2011).

\subsection{Treatment of psychiatric disorders}

Current treatment for PDs uses a combination of pharmacological and psychosocial therapies as these provide the best outcome (Kelly and Daley, 2013). However, pharmacologic treatments have limited efficacy across psychiatric diagnoses (Gaynes et al., 2009; Pittenger and Bloch, 2014; Widge and Moritz, 2016). For example in SCZ, about 20-30\% of patients are refractory to pharmacologic treatment and the same percentage is estimated to experience a relapse even after treatment (Patel et al., 2014). Pharmacological treatment are largely symptom alleviating, and may slow down disease progression (Kelly and Daley, 2013; Patel et al., 2014). However, some of the medications have been shown to cause side effects (Insel, 2009; Cuthbert and Insel, 2013). This may largely be attributed to the fact that most drugs work by targeting global brain circuits inadvertently disrupting function that improve some symptoms but cause side effects (Insel, 2012; Cuthbert and Insel, 2013; Patel et al., 2014). Side effects of psychotropic drugs can be severe. For example, antipsychotic drugs used for SCZ, can lead to motor disturbance (e.g. tremors reminiscent of parkinsonism), metabolic (such as weight gain and dyslipidemia) and associated 
hormonal changes which contribute to poor treatment compliance and ultimately relapse (Patel et al., 2014).

With the advancement in neuroscience and genetics, our understanding of PDs is changing while the unravelling of peculiar neural circuits involved in these disorders is providing new therapeutic windows (Insel, 2009, 2010a, 2010b, 2012; Krystal and State, 2014). Electric and magnetic deep brain stimulation techniques are proposed to help with the direct targeting of neural circuits associated with disease pathology (Widge and Moritz, 2016). The success rate of these new treatments are low still owing to the overall limited comprehension of disease aetiology and pathophysiology (Widge and Moritz, 2016). Because PDs are often chronic and difficult to cure, emphasis is placed on the provision of optimum support to patients and their families so they can cope and live with the disease with minimum distress (Kelly and Daley, 2013; Patel et al., 2014). Psychosocial interventions such as motivational interviewing, counselling, cognitive behaviour therapy, peer support, family education, and helping patients to hold a job among others are crucial for the reintegration of patients back into their communities (Kelly and Daley, 2013; Patel et al., 2014).

\subsection{Genetic overlap across psychiatric disorders}

The presence of shared symptoms and comorbidity among PDs have fueled the debate that these disorders have a common and overlapping aetiology (Burmeister, McInnis and Zöllner, 2008; Smoller et al., 2019) see figure 1. Advancement in large-scale genome analysis have further identified a number of shared genomic loci across several PDs giving credence to pleiotropic genetic influence (Cross-Disorder Group of the Psychiatric Genomics Consortium, 2013; Lee et al., 2013, 2019; Ruderfer et al., 2018; The Brainstorm Consortium et al., 2018).

The Psychiatric Genomics Consortium identified pleiotropic effects of genomic loci across five disorders - ASD, ADHD, SCZ, BP, and MDD (Cross-Disorder Group of the Psychiatric Genomics Consortium, 2013; Lee et al., 2013), which have recently been expanded to include three additional disorders: Tourette syndrome, obsessive-compulsive disorder (OCD), and anorexia nervosa based on analysis of even larger datasets (Lee et al., 2019). Shared genetic loci between SCZ and BP have been independently reported (Ruderfer et al., 2018). Furthermore, significant correlation of 
SCZ with ADHD, BP, and MDD have also been reported while the same study observed robust correlations across psychiatric disorders and phenotypic traits (cognitive and personality) (The Brainstorm Consortium et al., 2018).

\section{A}

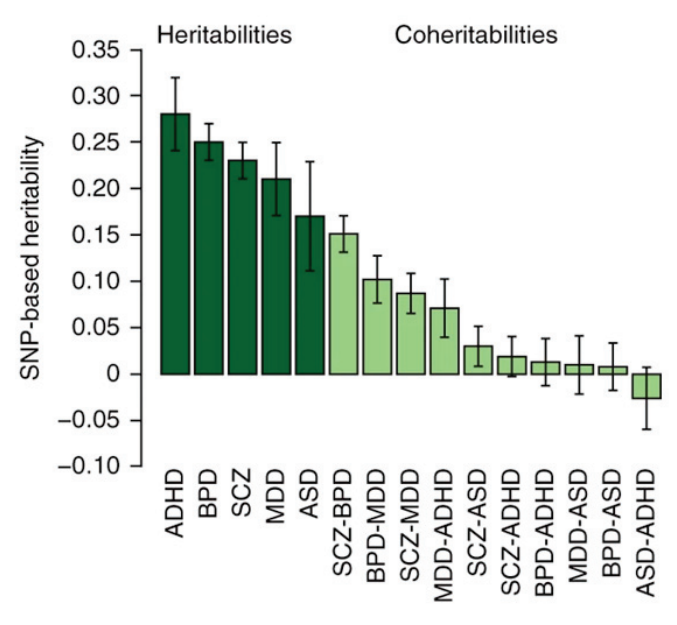

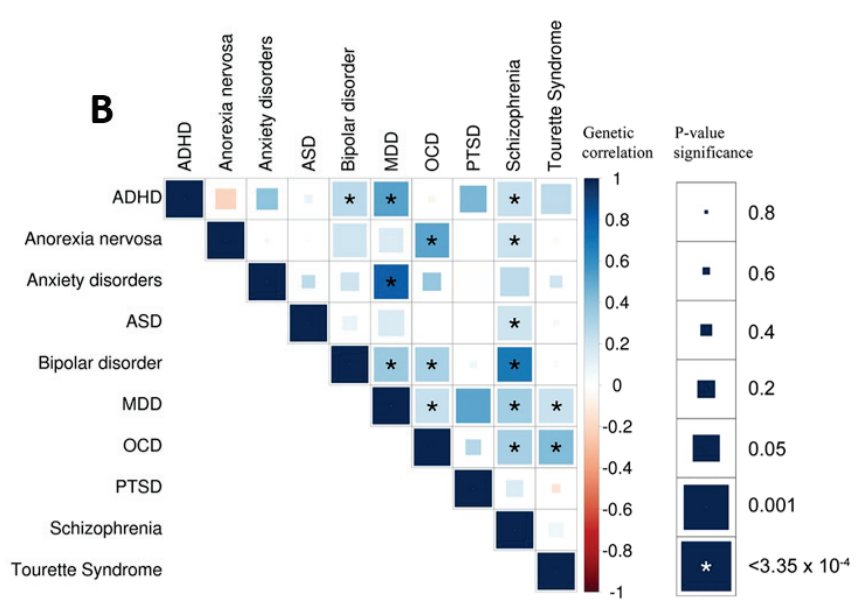

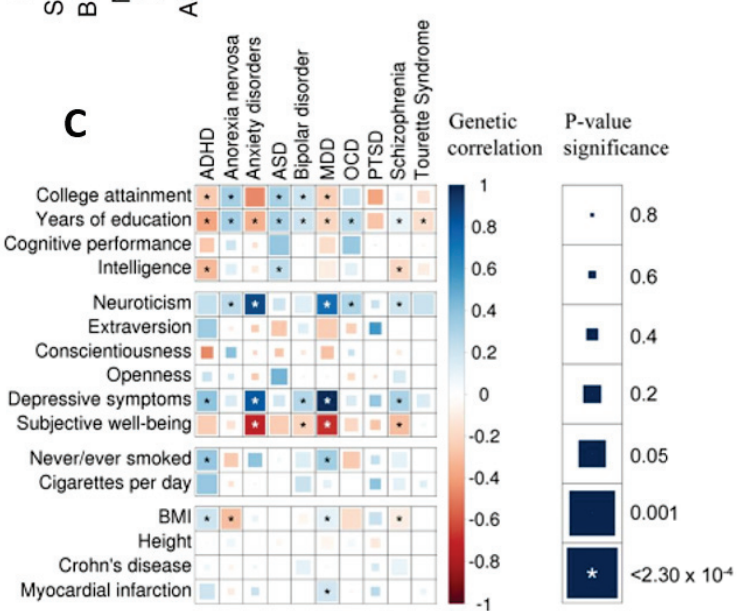

Figure 1. Shared correlations across psychiatric disorders. A) Genetic relationship across five disorders. The error bars represent the estimates \pm 1.96 s.e. Figure from Lee et al., 2013. B) Genetic relationship across ten disorders. C) Genetic relationship across ten disorders and phenotypic traits. The colour and size of the box indicates the extent of correlation and significance respectively. ADHD: attention deficit hyperactivity disorder, ASD: autism spectrum disorder, BPD: bipolar disorder, MDD: major depressive disorder, OCD: obsessive-compulsive disorder, PTSD: post-traumatic stress disorder, SCZ: schizophrenia. * represents genetic correlations 
significantly different from zero after Bonferroni correction. Figures $2 \mathrm{~B}-\mathrm{C}$ adapted from taken from The Brainstorm Consortium, 2018.

Some insights from the pleiotropic effects of genes suggest that the loci involved confer a general psychiatric risk by converging on pathways involved in early neural development and the formation of brain circuitry. Later in life, the generic alterations are fine-tuned by other genetic loci and environmental factors to express phenotypes reminiscent of the various disorders (Vorstman and Burbach, 2014; Guan et al., 2015; Lee et al., 2019). For instance, the shared loci between SCZ and BP have been reported to involve synaptic and neuronal pathways (Ruderfer et al., 2018).

Multiple methods have robustly identified risk-associated variation in specific channel subunits of voltage-gated calcium channels, as well as indicating a convergence of risk on calcium signalling more broadly (Cross-Disorder Group of the Psychiatric Genomics Consortium, 2013; Devor et al., 2017; Pardiñas et al., 2018). However, how these gene variants affect brain function to increase risk for these diseases is poorly understood.

\subsection{Voltage-gated calcium channels}

Voltage-gated calcium channels (VGCC) belong to a family of hetero-multimeric transmembrane proteins that are involved in the regulation of calcium entry into cells, gene expression and neurotransmitter release (Andrade et al., 2019). They are present in excitable cells of the peripheral and central nervous systems, secretory systems, cardiac and smooth muscle cells (Heyes et al., 2015; Andrade et al., 2019).

VGCC are made up of four subunits $(\alpha 1, \alpha 2-\delta, \beta$, and $\gamma)$ encoded by a family of related but distinct genes (Heyes et al., 2015). The $\alpha 1$ subunit forms the central pore, the voltage sensor, and the gating apparatus that facilitates the passage of calcium ions (Heyes et al., 2015). The three other subunits $\left(\alpha_{2}-\delta, \beta\right.$, and $\left.\gamma\right)$ modifies the properties of the VGCC by influencing the "anchorage, trafficking, gating, and inactivation behaviour" of the channel and sometimes interact with other proteins or channels to modulate their function (Heyes et al., 2015; Andrade et al., 2019). 
The $\alpha_{1}$ subunit is further divided into ten $\alpha_{1}$ subunit gene products $\left(\alpha_{1} \mathrm{~S}, \alpha_{1} \mathrm{C}, \alpha_{1} \mathrm{D}, \alpha_{1} \mathrm{~F}, \alpha_{1} \mathrm{~A}, \alpha_{1} \mathrm{~B}\right.$, $\alpha_{1} \mathrm{E}, \alpha_{1} \mathrm{G}, \alpha_{1} \mathrm{H}$, and $\left.\alpha_{1} \mathrm{I}\right)$ (Heyes et al., 2015). They can be classified into three sub-families (Cav1Cav3) based on their $\alpha_{1}$ subunit's sequence homology or into L-, N-, P-, Q-, R-, and T-type channels based on their electrophysiological and pharmacological properties (Heyes et al., 2015; Andrade et al., 2019). The assigned letters describe the observed feature of the calcium current for that channel. "L-type" refers to large calcium currents; "P/Q-type" is associated with calcium currents in Purkinje neurons, "N-type" refers to a calcium current that is neither L- nor T-type; "Rtype" refers to the resistance of these channels to pharmacological blockers of L-, P/Q-, and Ntype calcium currents; whilst the "T-type" refers to transient calcium currents (Dolphin, 2018). The L-type channels have a high activation threshold, large-conductance, and long open time. The N-, P-, Q-, and R-type have intermediate conductances and open time relative to the L-type, but possess activation thresholds ranging from medium to high. The T-type channels have a low activation threshold, low conductance, and short open time (Heyes et al., 2015; Dolphin, 2018; Andrade et al., 2019).

Whereas the Cav3 channels can form functional channels on their own, they sometimes form associate complexes with the other auxiliary subunits. On the other hand, both the Cav1 and Cav2 channels need to form a complex with one of the four- $\beta$ subunits or one of four $\alpha 2 \delta$ in order to form a functional channel (Heyes et al., 2015).

The $\alpha_{1}$ subunit consists of four homologous transmembrane domains (TMDI-IV), each with six transmembrane helical segments (S1-S6), amino and carboxyl termini see figure 2. The S4 is responsible for the channels' voltage sensing properties, which is facilitated by the positively charged arginine residues they contain. On the other hand, the S5 and S6 contain negatively charged glutamates and/or aspartates located on the re-entrant loop linking them and thus, responsible for channel conductivity and selectivity. The amino and carboxyl termini as well as the sequences that link the four homologous domains with each other are cytosolic in nature, which enables their interaction with other signalling proteins and/or cascades (Catterall, 2011; Zamponi et al., 2015; Andrade et al., 2019).

Due to the scope of this thesis, focus will only be placed on the LTCCs with emphasis on $\alpha 1 \mathrm{C}$ and $\alpha 1 D$. 


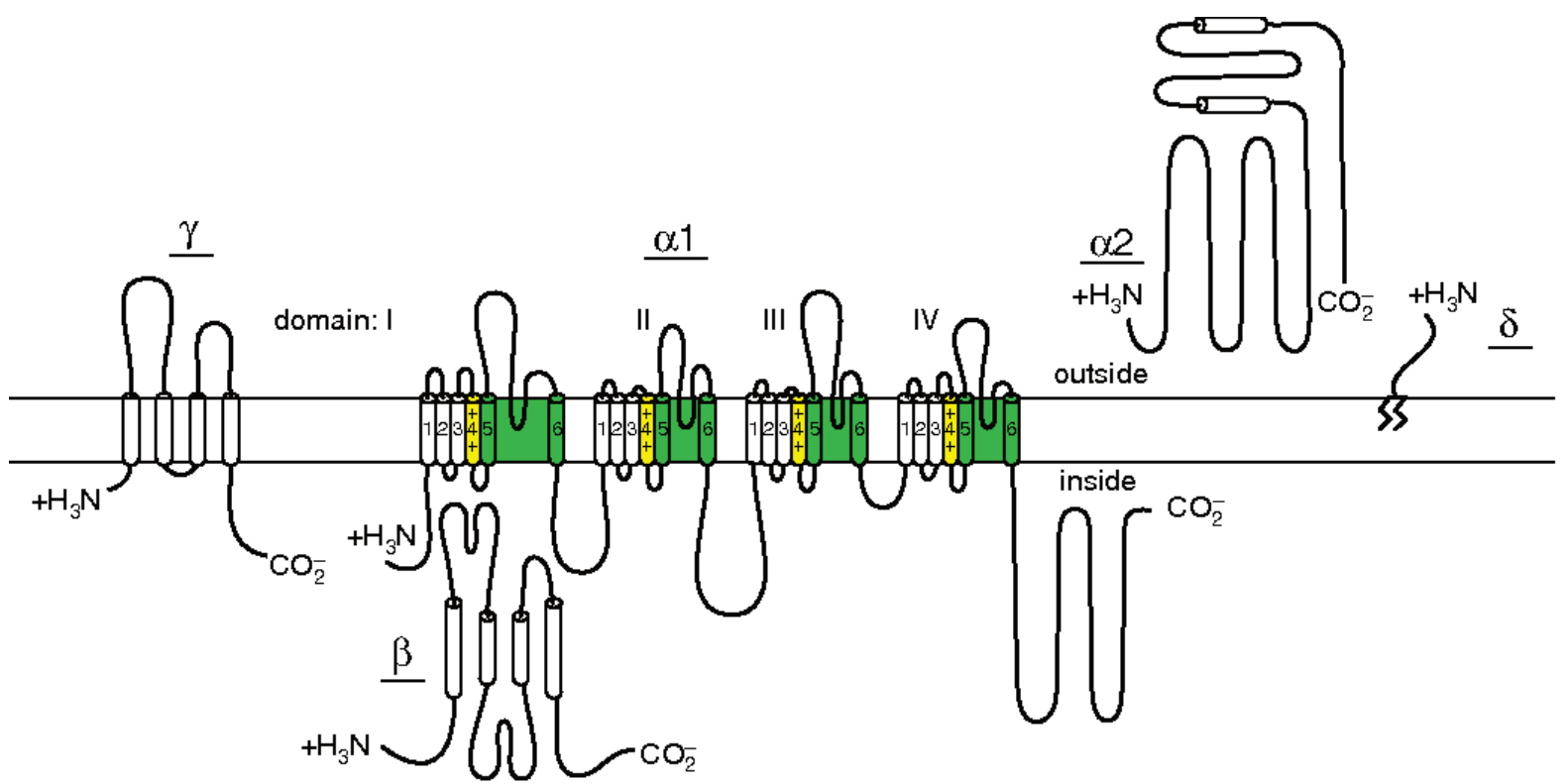

Figure 2. Schematic of the subunit composition of voltage gated calcium channels. The pore forming $\alpha 1$ subunit is composed of four homologous transmembrane domains (TMDI-IV), each with six helical segments (S1-S6), amino and carboxyl termini. The auxiliary subunits $\gamma, \beta$, and $\alpha 2-\delta$ interact with the principal subunit to modulate the properties of the channel. Figure from Caterall, 2011.

\subsubsection{L-type calcium channels (LTCCs)}

The LTCCs belong to the Cav1 sub-family of $\alpha_{1}$ subunits of VGCCs, and it is made up of the following four subtypes: $\mathrm{Ca}_{v} 1.1, \mathrm{Ca}_{\mathrm{v}} 1.2, \mathrm{Ca}_{\mathrm{v}} 1.3$ and $\mathrm{Ca}_{\mathrm{v}} 1.4$ encoded by CACNA1S, CACNA1C, CACNA1D and CACNA1F respectively (Heyes et al., 2015). Cav1 channels are classified as Ltype based on their electrophysical properties i.e. high activation threshold, large-conductance, and long open time (Heyes et al., 2015; Andrade et al., 2019). Pharmacologically, LTCCs are sensitive to dihydropyridines, phenylalkylamines, and benzothiazepines (Kabir, Martínez-Rivera and Rajadhyaksha, 2017) although it has been reported that at higher doses, phenylalkylamines can affect T-type VGCCs, potassium and sodium channels (Freeze, McNulty and Hanck, 2006).

Even though LTCCs are generally activated at high voltage thresholds, slight electrophysical differences exist between Cav1.2 and Cav1.3 (Andrade et al., 2019). Whereas Cav1.2 have higher 
activation threshold and slower inactivation, Cav1.3 have lower activation threshold and faster inactivation (Koschak et al., 2001; Tang et al., 2004; Heyes et al., 2015). Currently, no drugs have been shown to selectively block Cav1.2 or Cav1.3. Nevertheless, various studies report that Cav1.2 are more sensitive to the dihydropyridines, isradipine and nimodipine (Koschak et al., 2001; Xu and Lipscombe, 2001). More studies are being conducted to further characterise these LTCC subtypes with the hope of identifying unique features that can be harnessed for the development of selective subtype pharmacologic blockers (Zuccotti et al., 2011).

The Cav1.1 and Cav1.4 channels are not expressed in the brain, but are expressed in skeletal muscles and photoreceptors of the eye respectively (Heyes et al., 2015; Kabir, Martínez-Rivera and Rajadhyaksha, 2017; Andrade et al., 2019). Cav1.2 and Cav1.3 are the main LTCCs in the brain (neuronal LTCCs). Data from in situ hybridization analysis studies have shown significant overlap in the expression of Cav1.2 and Cav1.3 in the heart, brain, secretory system and smooth muscle (Andrade et al., 2019). The neuronal LTCC, Cav1.2 and Cav1.3 are located in the soma and synapses of neurons where they are involved in postsynaptic modulatory activities such as dendritic processing and gene transcription (Heyes et al., 2015). All four Cav1 genes undergo alternative splicing and are associated with the other auxiliary subunits (Andrade et al., 2019).

\subsubsection{Expression of $\mathrm{Ca}_{V} 1.2$}

Transcriptomic data (RNA-Seq of Consensus dataset) show that in the human brain, Cav1.2 mRNA is expressed in the cerebral cortex, cerebellum, olfactory region, hippocampal formation, amygdala, basal ganglia, thalamus, hypothalamus, midbrain, pons and medulla, corpus callosum, spinal cord, and retina of the human brain (Uhlen et al., 2015). The Allen Mouse brain in situ hybridisation (ISH) dataset show a similar expression of Cav1.2 (Lein et al., 2007). Immunohistochemical staining has shown Cav1.2 protein in the endothelial cells and neuropil of the cerebral cortex, Purkinje cells of the cerebellum, hippocampus, and caudate of both the human and mouse brain (Lein et al., 2007; Uhlen et al., 2015).

In zebrafish (Danio rerio), whole-mount ISH (WISH) analysis showed that Cav1.2 is expressed in the lateral plate mesoderm, primary germ layer, primitive heart tube and pancreas primordium in larvae aged $21-26 \mathrm{hpf}$ (Rottbauer et al., 2001). At 48 hpf, expression of Cav1.2 is found in the 
gut tube and the central nervous system (Rottbauer et al., 2001). From 72 hpf, expression in the ceratohyal cartilage and ventral mandibular arch (Ramachandran et al., 2013). RT-qPCR analysis showed that Cav1.2 mRNA was expressed both in the atrium and ventricle of adult zebrafish (Haverinen et al., 2018).

\subsubsection{Expression of Cav1.3}

In the human brain, Cav1.3 mRNA (based on RNA-Seq of Consensus dataset) has been detected in the cerebral cortex, olfactory region, midbrain, pons and medulla, corpus callosum, spinal cord, retina, cerebellum, as well as in the limbic and mesolimbic structures (amygdala, basal ganglia, hippocampus, thalamus, hypothalamus), which are enriched with dopaminergic neurons (Uhlen et al., 2015). Immunohistochemical staining of the human brain showed that Cav1.3 protein is expressed in the neuronal and glial cells of the cerebral cortex, cells in the granular layer of the cerebellum, hippocampus, and caudate (Uhlen et al., 2015). Similarly, in mice, Cav1.3 mRNA is expressed in the limbic and striatal areas (Hetzenauer et al., 2006; Lein et al., 2007).

There are two orthologues of Cav1.3 in the zebrafish, Cav1.3a and Cav1.3b (Sidi et al., 2004; Sanhueza et al., 2009). In the larval zebrafish brain, WISH revealed the expression of Cav1.3a mRNA as early as $24-30$ hours post-fertilisation (hpf) in the telencephalon, thalamic and hypothalamic diencephalon, midbrain, and ventral hindbrain (Sidi et al., 2004; Sanhueza et al., 2009). From 48 hpf onwards, Cav1.3a expression was even more pronounced in nearly the entire brain (Sidi et al., 2004; Sanhueza et al., 2009). In 5 days post fertilisation (dpf) larvae, Cav1.3a mRNA was present in the eye (although at low levels) and ear, while Cav1.3b mRNA was evident in the otic vesicle (Sidi et al., 2004). RT-qPCR analysis showed that Cav1.3a mRNA was expressed both in the eyes and ears of the adult zebrafish (Sidi et al., 2004).

\subsubsection{Calcium signalling pathways}

LTCCs have calmodulin (CaM)-binding sites in their carboxy-terminal tails through an IQ motif as well as a site in the distal parts of the carboxy-terminal where calcineurin could bind to facilitate downstream signalling cascades (Catterall, 2011) see figure 3. Influx of calcium via NMDA-Rs 
and LTCCs can lead to the activation of the calcium sensor CaM. CaM causes the activation of $\mathrm{Ca}^{2+} / \mathrm{CaM}$-dependent protein kinases (CaMKs) and the Ras/MAPK pathway that drives the activation of cyclic-AMP-response-element-binding protein (CREB) (West, Griffith and Greenberg, 2002; Barbado et al., 2009). Genes activated by the CREB play important roles in neuronal, synaptic, and behavioural plasticity (Kabir, Martínez-Rivera and Rajadhyaksha, 2017). In response to different stimuli, the differential site phosphorylation of the CREB can lead to it forming alternative cofactor complexes to activate the transcription of specific genes such as $C$ FOS and BDNF (West, Griffith and Greenberg, 2002).

C-FOS is an immediate early gene that encodes transcription factors to activate downstream genes and is often used as a surrogate to measure neuronal activity (Velazquez, Caputto and Boussin, 2015). Increased expression of C-FOS is associated with neuronal activation (Velazquez, Caputto and Boussin, 2015). C-FOS expression has been linked to learning and memory (Gallo et al., 2018), seizure generation (Malhi et al., 2014; Barros et al., 2015; Gawel, Kukula-Koch, et al., 2020), and PDs (Gallo et al., 2018). BDNF is a neurotrophic factor, important for neurogenesis, neuronal maturation, and synaptic plasticity (Favalli et al., 2012). Unlike BDNF, other stimuli such as the elevation of intracellular cAMP through receptor tyrosine kinases (activated by growth factors) and G-protein-coupled receptors (activated by neuromodulators) can activate the expression of C-FOS (West, Griffith and Greenberg, 2002)). In addition, there is evidence to suggest that LTCCs, and not NMDA-Rs preferentially activate BDNF (Ghosh, Carnahan and Greenberg, 1994; Tabuchi et al., 2000). Furthermore, LTCCs may also activate calcineurin, a calcium/calmodulin-dependent protein phosphatase that regulates the transcription by nuclear factor of activated T cells (NFAT) and nuclear factor-B (NF-B) (West, Griffith and Greenberg, 2002; Barbado et al., 2009). 


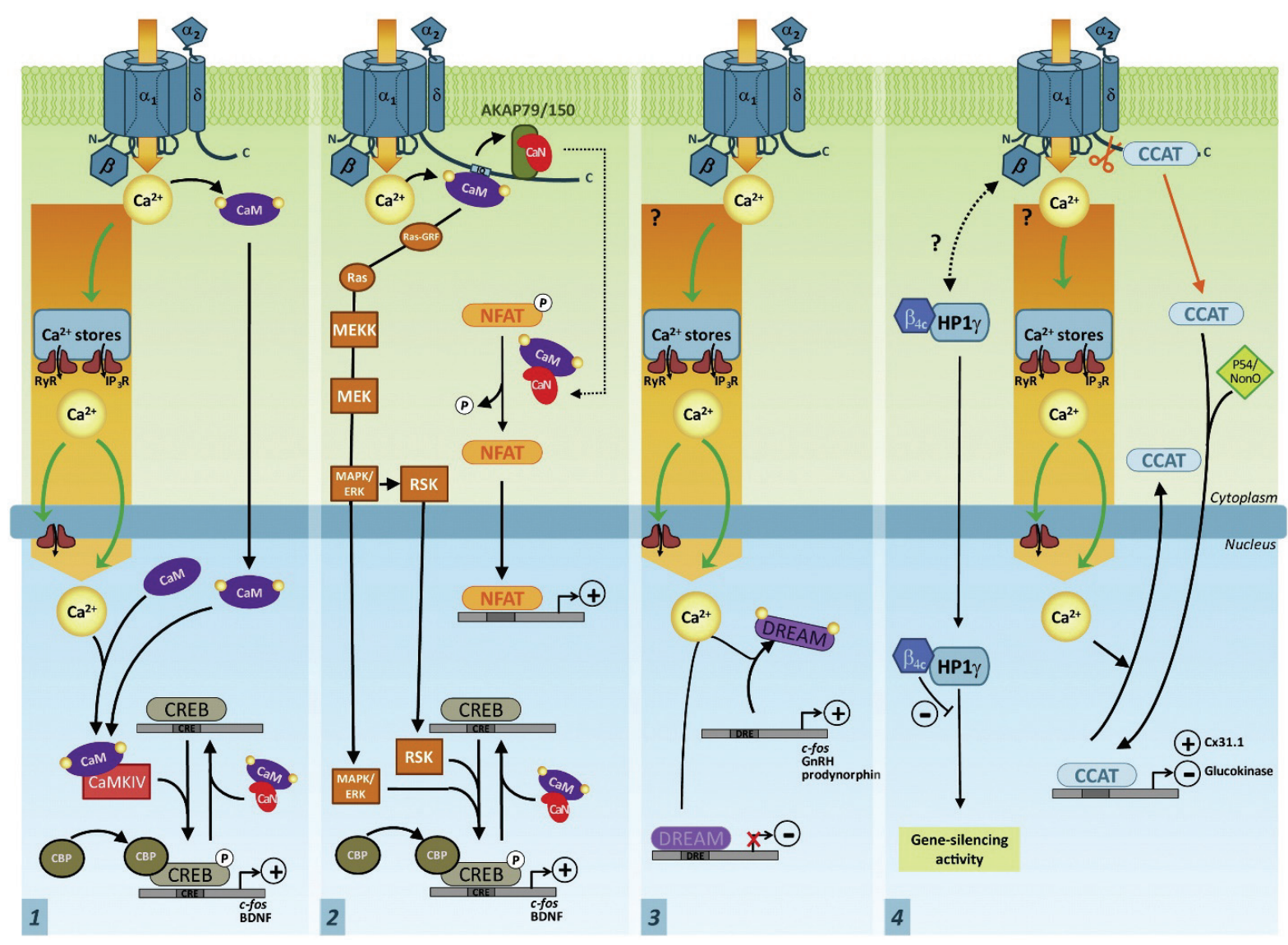

Figure 3. Schematic representation of gene transcription pathways regulated by voltage gated calcium channels. Panel 1 shows the example of CREB phosphorylation by calcium binding proteins. Panel 2 shows the examples of two transcription factors, MAPK and NFAT that are regulated by the calmodulin/IQ and calmodulin-activated calcineurin calcium channel signalling complexes respectively. Panel 3 shows the example of transcription factor DREAM. Panel 4 shows instances when calcium channel subunits (e.g., $\beta_{4 c}-$ splice variant of the $\beta_{4}$ subunit) or calcium channel fragments (e.g., CCAT - derived from the c-terminus of LTCCs to form a transcription factor) can act as transcription factors to regulate gene activity. Figure from Barbado et al., 2009.

\subsubsection{Association of L-type calcium channels with psychiatric disorders}

Earlier linkage studies in large pedigrees were used to identify specific genomic regions that cosegregate with PDs such as SCZ and BP. However, positive linkage findings in relation to SCZ and BP were subsequently discovered not to be persuasive. Later, lots of evidence emerged that 
these disorders are highly complex and polygenic. Moreover, although the earlier positive linkage findings could in fact contain disease susceptible variants, they are weak on their own but can have effects if measured within a large population (Burmeister, McInnis and Zöllner, 2008). Genomewide association studies (GWAS) have influenced the genetics landscape of diseases by heralding the identification of single nucleotide polymorphisms (SNPs) that happen across the genome and are associated with increasing the risk of PDs. GWAS analysis is often carried out on a large-scale basis. Variants in genes involved in neuronal LTCCs (CACNA1C and CACNA1D) have been found by various GWAS to increase the risk for PDs such as SCZ, BP, and ASD (Cross-Disorder Group of the Psychiatric Genomics Consortium, 2013; Ripke et al., 2014; Heyes et al., 2015; Kabir, Martínez-Rivera and Rajadhyaksha, 2017).

\subsubsection{Cav1.2 and psychiatric disorders}

Variations in the genes that encodes for Cav1.2, CACNA1C, have been found to be associated with an increased risk of BD, SCZ, MDD, ADHD, and ASD (Cross-Disorder Group of the Psychiatric Genomics Consortium, 2013; Ripke et al., 2014). In this area, a well-replicated SNP was the association of rs1006737 (located in intron 3 of the CACNA1C gene) with BP (The Wellcome Trust Case Control Consortium, 2007; Ferreira et al., 2008; Sklar et al., 2008). Subsequently, rs1006737 was linked to SCZ, ASD, MDD as well as ADHD (Nyegaard et al., 2010; Wellcome Trust Case Control Consortium et al., 2010; Li et al., 2015). Several other SNPs in the CACNA1C have since been found to confer a greater risk for developing various PDs (Cross-Disorder Group of the Psychiatric Genomics Consortium, 2013; Purcell et al., 2014; Roussos et al., 2014).

Interestingly, most of the SNPs are located within the non-coding regions of genes (Harrison, 2015). In silico analysis predict that they are located within promotor and enhancer regions and can therefore interfere with gene expression (Roussos et al., 2014). For instance, functional studies reveal that the rs 1006737 leads to alterations in the expression of CACNAIC in human carriers (Bigos et al., 2010; Gershon et al., 2014) as well as in induced human neurons (Yoshimizu et al., 2015). In addition, broader phenotypic spectrum such as impairments in latent inhibition, prepulse inhibition (PPI) and sleep disturbance have been observed in some human rs1006737 SNP carriers (Harrison, 2015; Andrade et al., 2019). CACNA1C SNPs are known to have shared effects across multiple disorders, therefore, suggesting the shared genetic effects of these disorders (Cross- 
Disorder Group of the Psychiatric Genomics Consortium, 2013). Thus, necessitating the need to investigate the functional roles of these SNPs in relevant disease phenotypes rather than focusing on a rigid disease classification.

Missense mutations in the CACNAIC is known to cause Timothy syndrome via a gain-of-function mechanism (Napolitano and Antzelevitch, 2011; Moon et al., 2018). Timothy syndrome (TS) is an autosomal dominant disease characterised by webbing of the fingers and toes, craniofacial dysmorphologies and cardiac defects (Napolitano and Antzelevitch, 2011). The cardiac defects is a prolongation of the QT wave leading to arrhythmias and sudden death. In some TS patients, symptoms of autism, impaired cognitive functions and seizures are also reported (Napolitano and Antzelevitch, 2011). Type I, also called the classical type is caused by mutations in exon $8 \mathrm{a}$ (an alternative transcript) of the gene - where ASD typically coexists in patients (Splawski et al., 2004, 2005). The type II, also called the atypical type is caused by mutations in exon 8 - where patients additionally exhibit other PDs such as OCD, depression and BP (Splawski et al., 2005; Gershon et al., 2014). Recently, rare mutations in regions outside exons 8 and 8 a have also been identified. These rare TS involve mutations in exons 12 (Wemhöner et al., 2015), 27 (Boczek et al., 2015), 33 (Bozarth et al., 2018), and 36 (Gillis et al., 2012) as well as in intron 28 (Bozarth et al., 2018). Persons with TS do not survive past childhood hence, it was interesting, when a study reported that a TS patient, who survived to adulthood, developed BP (Gershon et al., 2014).

In addition, two de novo missense variations in CACNA1C were associated with SCZ in a Swedish population (Purcell et al., 2014). The prediction is that the (G/T) risk variant leads to an alteration in a donor splice site of exon 21 , while the second risk variant $(\mathrm{C} / \mathrm{T})$ leads to an early stop codon in the intracellular linker between TMDIII and TMDIV (Heyes et al., 2015; Andrade et al., 2019).

\subsubsection{Cav1.3 and psychiatric disorders}

Similarly, SNPs in CACNA1D are known to be associated with an increased risk of developing PDs (Cross-Disorder Group of the Psychiatric Genomics Consortium, 2013; Ripke et al., 2014). The SNP rs893363 is associated with BP, SCZ, ASD, ADHD, and MDD (Cross-Disorder Group of the Psychiatric Genomics Consortium, 2013; Pardiñas et al., 2018). One study found other noncoding variants linked to BP (Ament et al., 2015) while two variants in the coding region (A1751P 
and R1771W) have also been linked to BP (Ross et al., 2016). A study in the Han Chinese population reported no linkage of CACNA1D with SCZ (Ross et al., 2016), however subsequent studies are reported to have found such an association (Devor et al., 2017; Andrade et al., 2019).

Furthermore, whole-exome sequencing revealed de novo variants in the coding region of CACNA1D (Andrade et al., 2019). These variants are associated with a predisposition to ASD and intellectual disability, depression, anxiety, fear and seizures (Pinggera et al., 2015, 2017). With the exception of one variant, all the other variants are reported to cause a gain of function (GOF) of Cav1.3 channels (Andrade et al., 2019).

In humans, missense mutations in the Cav1.3 channel leading to hypersensitive channels as a result of GOF effects have been reported giving rise to novel insights to the function and pathology of the channel (Scholl et al., 2013; Pinggera et al., 2015, 2017; Pinggera and Striessnig, 2016). Human GOF mutations lead to a syndrome called primary aldosteronism, seizures and neurological abnormalities (PASNA) - some of the neurological abnormalities in PASNA includes global developmental delays and intellectual disabilities (Scholl et al., 2013). Additionally, ASD has been reported to be part of the GOF disease list (Iossifov et al., 2012; O'Roak et al., 2012; Pinggera et al., 2015). A genetic analysis of an epilepsy data set revealed that some patients carried mutations in the CACNA1D (Klassen et al., 2011). Similarly, analysis of exon sequencing data set of ASD also revealed mutations in the CACNA1D - some confirmed in publicly available datasets (De Rubeis et al., 2014). Furthermore, missense mutations resulting in loss of function (LOF) of the Cav1.3 channel have been reported in humans presenting a syndrome called sinoatrial node dysfunction and deafness (SANDD) (Baig et al., 2011). The phenotype of the LOF mutation is largely sensory and syndromic in nature.

\subsection{Phenotypic domains associated with LTCCs: Evidence from animal models}

LTCCs and in particular Cav1.2 contribute to the proper functioning of the brain circuitry involving the mesolimbic system, hippocampus, and amygdala, which are implicated in the pathophysiology of various psychiatric disorders (Bhat et al., 2012; Zamponi, 2016). The mesolimbic pathway involves the circuitry between the ventral tegmental area (VTA) and the 
nucleus accumbens (NAc), with modulation from the hippocampus, medial prefrontal cortex, and amygdala (Bhat et al., 2012).

Most of the functional roles of $\mathrm{Ca}_{\mathrm{v}} 1.2$ and $\mathrm{Ca}_{\mathrm{v}} 1.3$ in psychiatric disorders have been obtained from genetic mouse models and to a lesser extent, using zebrafish genetic models (Thyme et al., 2019; Banono et al., 2021). Studies have demonstrated that global deletion of Cav1.2 channels is embryonic lethal in rodents (Seisenberger et al., 2000), and zebrafish (Thyme et al., 2019). On the other hand, the global deletion of Cav1.3 results in hearing loss (Platzer et al., 2000; Baig et al., 2011).

\subsubsection{Evidence from rodent models}

\subsubsection{Addiction}

Addiction is a common comorbidity with BD and SCZ. Evidence from mice suggests that Cav1.2 and Cav1.3 channels play some roles in drug seeking behaviour, a behavioural trait linked to addiction (Kabir, Martínez-Rivera and Rajadhyaksha, 2017). The VTA - Nac pathway plays an important role in reward and motivation behaviours and it is the pathway that mediates the sensitization of the brain to psychostimulants such as cocaine, methamphetamine and morphine, of which Cav1.2 channels are reported to play important roles (Bhat et al., 2012). For instance, in cocaine sensitized rats, increased total protein levels and cell surface expression of Cav1.2 channels in pyramidal neurons in deep layers of the medial prefrontal cortex were reported (Ford, Wolf and Hu, 2009). Similarly, in mice dependent on methamphetamine, cocaine, and morphine, the levels of both Cav1.2 and Cav1.3 protein were increased in their frontal cortex and the limbic areas (Shibasaki, Kurokawa and Ohkuma, 2010). Furthermore, various studies showed that different LTCC antagonists affected drug seeking behaviours of rats in the conditioned place preference (CPP) tests (Calcagnetti and Schechter, 1992; Pucilowski, 1995; Biała, 2003; Chartoff, Pliakas and Carlezon, 2006). Isradipine suppresses the intake of sweetened water (Calcagnetti and Schechter, 1992), amphetamine has reinforcing effects of rats in the CPP test (Pucilowski, 1995), nimodipine suppresses the effect of nicotine (Biała, 2003) while the rewarding effects of cocaine was augmented when D-cis-diltiazem (LTCC antagonist) was injected in the ventral NAc shell of rats (Chartoff, Pliakas and Carlezon, 2006). 


\subsubsection{Fear and anxiety}

Anxiety is described as the feeling of apprehension to a harmless stimulus (Zamponi, 2016). Although a natural response, constant feeling of anxiety is a function of impaired neuronal function and is often a comorbidity of several PDs (Zamponi, 2016; Kabir, Martínez-Rivera and Rajadhyaksha, 2017). Patients with anxiety disorders often have alterations in their fear responses (Kabir, Martínez-Rivera and Rajadhyaksha, 2017). In line with the aforementioned, increased anxiety-like behaviour have been reported in mice with 1) heterozygous Cav1.2 knockout (Bader et al., 2011; Kabitzke et al., 2018), 2) global Cav1.2 haploinsufficiency, and 3) conditional forebrain and prefrontal cortex Cav1.2 knockout (Lee et al., 2012; Kabir et al., 2017). In contrast, Moosmang et al., did not observe any alteration in anxiety-like or exploratory phenotypes in conditional forebrain knockout of Cav1.2 (Moosmang et al., 2005). Another study reported that only females with heterozygous Cav1.2 knockout showed increased anxiety-like behaviour while their male counterparts exhibited normal behaviour (Dao et al., 2010).

Pharmacologically, the LTCC antagonists, nifedipine and nimodipine exerted anxiogenic effects in rats in the plus-maze (Viveros et al., 1996). In mice, nifedipine, flunarizine, verapamil and diltiazem blocked the anxiogenic effects of d-amphetamine (Biała and Kruk, 2007). The anxiogenic effects of LTCC in mice were only observed at high dosage administration of nifedipine and verapamil in mice. Otherwise, nifedipine ordinarily induced anxiolytic effects in the mice (Fulga and Stroescu, 1997; Zamponi, 2016).

Anxiolytic-like phenotypes have been reported in Cav1.3 knockout mice (Busquet et al., 2010) although there is the suggestion that these phenotypes may be a consequence of their hearing deficits (Platzer et al., 2000). Interesting, selective pharmacological activation of Cav1.3 channels in Cav1.2DHP ${ }^{-/}$mice with BayK8644 resulted depressive-like behaviours (Kabir, MartínezRivera and Rajadhyaksha, 2017; Andrade et al., 2019). It was possible to selectively target the Cav1.3 channels because the Cav1.2 of the $\mathrm{Ca}_{v} 1.2 \mathrm{DHP}^{-/-}$mice were mutated such that they became resistant to dihydropyridines.

The amygdala plays a central role in the regulation of fear circuits (Moosmang et al., 2005; Bhat et al., 2012) and its function is known to be modulated by LTCCs. Also, evidence from the 
Cav1.2DHP ${ }^{-/-}$mice treated with BayK8644 suggests that Cav1.3 perform a consolidation role in context-dependent fear memory but are neither necessary in the acquisition or extinction of conditioned memory (Andrade et al., 2019). In fear-conditioned rats whose startle responses were blocked by nimodipine, an increase in the expression of Cav1.2 channels were reported (Zamponi, 2016). When Cav1.2 in Nestin expressing cells were ablated, the acquisition of conditioned fear was reduced (Langwieser et al., 2010), while the deletion of Cav1.2 in the anterior cingulate cortex resulted in impaired observational fear (Jeon et al., 2010).

\subsubsection{Learning and memory}

Cav1.2 and Cav1.3 channels are involved in several processes relevant to psychiatric disorders such as learning, memory, and brain development (Kabir, Martínez-Rivera and Rajadhyaksha, 2017; Moon et al., 2018). LTCCs play a vital role in gene expression through the CREB and BDNF pathway that strongly facilitate learning and memory processes (Moon et al., 2018). Modulation of synaptic plasticity, an important phenomenon in learning and memory have also been shown to involve LTCCs (Moon et al., 2018).

Using conditional knockout mice, studies have shown the involvement of Cav1.2 channels in spatial memory and remote spatial memory consolidation (Moosmang et al., 2005; White et al., 2008). For instance, Moosmang and colleagues reported loss of Shaffer collateral/CA1 late-phase long-term potentiation (LTP), impairment of hippocampus-dependent spatial memory in the Morris water maze and labyrinth maze tests in a mouse line with an inactivation of the Cav1.2 channel in the hippocampus and neocortex (Cav1.2(HCKO)). Further analysis revealed the Cav1.2(HCKO) mice had decreased activation of the MAPK pathway and reduced cAMP response element (CRE)-dependent transcription in their CA1 pyramidal neurons (Moosmang et al., 2005). In another study, using mice with inactivated Cav1.2 in their principal hippocampal neurons, they showed that Cav1.2 channels regulate excitability of CA1 pyramidal neurons (Lacinova et al., 2008). Again, mice with conditional knockout of Cav1.2 in the hippocampus and cortex displayed impairments in spatial memory after 30 days of training in the Morris water maze (White et al., 2008). Additionally, another study found that LTCC antagonists reduced induction of LTP in the CA1 of the rat hippocampus (Freir and Herron, 2003). 
Finally, Cav1.3-deficient mice have impaired object location memory in a discrimination test, but no observed deficit was observed when they were tested in a Morris Water Maze test (Marschallinger et al., 2015). All the above studies suggest a critical role of these channels in gene expression, synaptic plasticity and learning and memory.

\subsubsection{Social behaviour}

To the best of our knowledge, very little experiments have been performed to unravel the roles of Cav1.2 and Cav1.3 in social behaviour (Kabir, Martínez-Rivera and Rajadhyaksha, 2017). Cav1.2 GOF mouse model displayed decreased preference for a social object in an automated social homecage test (Bader et al., 2011). Furthermore, when the Cav1.2 channels in glutamatergic neurons of the forebrain were deleted in mice, prefrontal cortex dependent social behaviour was impaired (Kabir et al., 2017). Mice that had their Cav1.3 channel pharmacologically activated with BayK8644 showed impaired social behaviour as a result of altered dopaminergic function in the VTA proposing a role for GOF mutations in impaired behavioural function (Andrade et al., 2019).

\subsubsection{Evidence from zebrafish models}

Unlike in rodents, very limited studies have been conducted in zebrafish on the effect of mutations in neuronal LTCCs on brain function. Recently, Thyme and colleagues generated cacnalc mutants via CRISPR/Cas9 to investigate the gene's role(s) in brain function. Homozygous mutants were characterised with pericardial oedema and died by $6 \mathrm{dpf}$. Hence, they performed most of their experiments using $4 \mathrm{dpf}$ larvae. The researchers found heterozygote mutants to have increased forebrain activity while homozygous mutants at $4 \mathrm{dpf}$ exhibited a plethora of behavioural differences from wild type (WT) ranging from impairments in the magnitude of startle or PPI response, light/dark activity and swimming behaviour (Thyme et al., 2019). On the other hand, homozygous cacnalda nonsense mutants display a classical auditory-vestibular phenotype reminiscent of "circler mutants" (i.e., circular swimming due to impaired balance) (Granato et al., 1996; Nicolson et al., 1998; Sidi et al., 2004). 


\subsubsection{Relevant behavioural tests in zebrafish for modeling psychiatric phenotypes}

There are several behavioural tests relevant to PDs that have been adapted to zebrafish (Burgess and Granato, 2007b, 2007a; Miller and Gerlai, 2007; Best et al., 2008; Schnörr et al., 2012; Magno et al., 2015). The presence of these phenotypic tools make zebrafish suitable for genetic and pharmacological analysis of psychiatric disease models as well as for phenotypic drug screening (Kokel and Peterson, 2008; Rihel et al., 2010; Bruni et al., 2016).

\subsubsection{Locomotor Behaviour}

Locomotion in zebrafish is a complex behaviour produced by the collective action of muscles and various neuronal circuitry along with neuromodulators in the brain (Norton, 2012). At approximately 17 hours post fertilization (hpf), the first spontaneous side-to-side coiling movements appear and by $27 \mathrm{hpf}$, the zebrafish embryos become sensitive to touch and slowswimming behaviour begins and develops with age. By 4- to $5 \mathrm{dpf}$, a swim bladder becomes prominent and larvae display mature swimming behaviour (Norton, 2012; Basnet et al., 2019). Several different neurotransmitters such as serotonin, dopamine, GABA, glutamate, and glycine are known to fine-tune locomotor behaviour as larvae mature (Norton, 2012).

The precise analysis of locomotor activity in zebrafish larvae using high-throughput methods have facilitated large-scale pharmacologic and mutant screens resulting in the identification of neuroactive compounds and a number of genes involved in the formation and modulation of neurocircuitry of locomotion (Rihel and Schier, 2012). For example, larval locomotor activity may be measured as the distance moved while features such as velocity, displacement and trajectory (Burgess and Granato, 2007a). Hypolocomotion is often associated with sedation, motor defects such as akinesia (Kalueff et al., 2013) or models of ASD (Sakai, Ijaz and Hoffman, 2018). Hyperlocomotion on the other hand, is typically associated with epilepsy (Afrikanova et al., 2013; Baraban, Dinday and Hortopan, 2013; Kalueff et al., 2013; Gawel, Kukula-Koch, et al., 2020; Tiraboschi et al., 2020), anxiety (Maximino et al., 2010; Kalueff et al., 2013) or the positive symptoms of SCZ (Seibt et al., 2010, 2011; Kalueff et al., 2013). Locomotor-associated behaviours such as the light-dark test, startle response and thigmotaxis have been used to model 
aspects of various brain disorders in larval and adult zebrafish (Kalueff, Stewart and Gerlai, 2014; Sakai, Ijaz and Hoffman, 2018; Basnet et al., 2019).

\subsubsection{Light-dark test}

The light-dark locomotion test is a measure of the locomotor activity and movement pattern of zebrafish larvae when they are exposed to alternating light and dark conditions after a period of acclimation (Basnet et al., 2019). The distance travelled and the movement pattern of zebrafish larvae in each of the conditions is assessed. Depending on the illumination transition i.e. light to dark transition or dark to light transition, the movement pattern of larvae will differ. Larvae increase their locomotor activity during a light-dark transition but decrease their locomotion when the illumination transitions from dark to light (Basnet et al., 2019; Gawel, Turski, et al., 2020; Kedra et al., 2020; Banono et al., 2021). Exaggerated reactivity in the light-dark test has been used as a surrogate measure of anxiety and/or stress (Kalueff et al., 2013; Stewart et al., 2014; Basnet et al., 2019; Kedra et al., 2020). Because the light-dark test is dependent on the integrity of the development and function of the brain and the visual system, the test can be used to further the understanding of neurobehavioural effects of drugs and genes. For instance, the light-dark test has been used to extract the pharmacological profiles of a number of dopaminergic drugs (Irons et al., 2013).

\subsubsection{Thigmotaxis}

Thigmotaxis is the inclination of an animal to avoid the centre of an open-field while preferring to stay in close proximity with the wall of an open-field - this tendency is commonly referred to as "wall-hugging" behaviour (Schnörr et al., 2012). Thigmotaxis is an evolutionarily conserved phenomenon across different species and it is used as a measure of anxiety (Schnörr et al., 2012; Kalueff et al., 2013). It is common to see animal exhibiting thigmotactic behaviour when they move into a novel environment (Schnörr et al., 2012). Larval zebrafish with thigmotaxis stay close to the wall of multi-well plates or petri dishes as early as $5 \mathrm{dpf}$ (Schnörr et al., 2012; Basnet et al., 2019). Anxiolytic drugs, such as diazepam have been shown to attenuate thigmotaxis while anxiogenic drugs, such as caffeine and pentylenetetrazole have been shown to enhance thigmotaxis 
in zebrafish larvae (Schnörr et al., 2012; Kalueff et al., 2013; Basnet et al., 2019). In zebrafish, besides being used as a measure of anxiety and fear, thigmotaxis is sometimes used as a surrogate measure of the repetitive behaviour observed in ASD (Maximino et al., 2010; Kalueff et al., 2013; Stewart et al., 2014; Sakai, Ijaz and Hoffman, 2018).

\subsubsection{Startle Response}

The startle response is a conserved feature in all animals in reaction to startle stimuli (Basnet et al., 2019). In zebrafish, the "brainstem escape network" which are a bunch of reticulospinal neurons (RCN) comprising of Mauthner cells (M-cells), homologues of MiD2 $\mathrm{cm}$ and MiD3cm and other neurons adjacent to the M-cells (Norton, 2012; do Carmo Silva, Lima-Maximino and Maximino, 2018; Basnet et al., 2019). The RCN receive visual, acoustic, and tactile stimuli (Norton, 2012; Kalueff et al., 2013). The startle response could be characterised as a sudden increase in the velocity and acceleration of movement/swimming when triggered by either visual, acoustic, and tactile stimuli (Best et al., 2008; Norton, 2012). However, when exposed to the same stimulus multiple times, zebrafish can habituate to the stimulus, resulting in a decrease in rapid movements (Norton, 2012).

When the M-cells are activated by an acoustic stimulus, a robust turn that leads to a fast response called C-start is formed with a latency of about $5 \mathrm{~ms}$ (Burgess and Granato, 2007b; do Carmo Silva, Lima-Maximino and Maximino, 2018). In response to dark flashes, zebrafish display the Obend (Norton, 2012). The latency for a visual stimuli response ranges between 10-20 ms and up to a few hundred ms (do Carmo Silva, Lima-Maximino and Maximino, 2018). The tactile, visual and acoustic startle response (ASR) is present as early as 2-, 3-, and 5 dpf respectively (Burgess and Granato, 2007b, 2007a; Best et al., 2008; Colwill and Creton, 2011). Interesting, it has been suggested that M-cell control the C-start but not O-bend (Norton, 2012).

When a startle stimulus is preceded by a weak non-startling stimulus called the prepulse, the probability or intensity of displaying a startle response decreases in a phenomenon referred to as PPI (Burgess and Granato, 2007b; Banono and Esguerra, 2020). PPI is a quantitative measurement of sensorimotor gating and it is a useful endophenotype for a range of psychiatric and neurological disorders (Burgess and Granato, 2007b; do Carmo Silva, Lima-Maximino and Maximino, 2018). 
Similar to human and rodent studies, zebrafish PPI can be pharmacologically modulated apomorphine (dopamine agonist) decreases PPI, while haloperidol (dopamine antagonist) increases PPI and ketamine (NMDA-R antagonist) differentially modulates PPI based on the inter stimulus interval (Burgess and Granato, 2007b). Thus, PPI can be used to provide information about the integrity of brain circuitry responsible for ensuring normal sensorimotor function (Burgess and Granato, 2007b). In general, the startle response tests are useful in studying escape and avoidance behaviours in the context of fear and anxiety in zebrafish larvae (Colwill and Creton, 2011).

\subsubsection{Shoaling}

Zebrafish are a social species that exhibit a preference for conspecifics and start shoaling early on in development. They prefer to spend most of their time with conspecifics, and this behavioural feature can be exploited in various fields of behavioural study. Shoaling and social behaviour in general, can be disrupted by a plethora of environmental, developmental, genetic, and chemical factors. It offers many possibilities for the characterisation of aberrant behaviour, and for the screening of drug targets. The shoaling assay can be used to model disorders such as SCZ, ASD or locomotor deficits (Geng and Peterson, 2019).

The following social dynamics: nearest neighbour distance (NND), inter-individual distance (IID), polarization and global speed of the shoal can be measured when performing the shoaling assay (Miller and Gerlai, 2012). The NND measures the distance of one animal to its closest neighbour while the IID measures the mean distance between a reference animal and all other animals of the group. Polarization measures the extent to which members of the group move in the same direction whereas global speed gives an indication of the motion characteristics of the members of the group (Miller and Gerlai, 2012).

\subsection{Limitations of the zebrafish as a model organism}

The zebrafish brain develops through the process of eversion compared to the invagination process of their mammalian counterparts, which results in distinct differences in the gross anatomical structure of the zebrafish (Wullimann and Mueller, 2004). This can sometimes pose a challenge 
when drawing comparisons between observations made in zebrafish brains with those of mammalian models and humans. Moreover, further challenges are met when comparing embryonic or larval brain and behavioural phenotypes and "transposing" these to postnatal or adult pathologies and behaviours. In addition, the zebrafish, like other teleost species, has undergone gene duplication during evolution - a phenomenon where one mammalian gene has two zebrafish orthologues. Although the duplicated genes often encode for proteins with similar or significant overlapping functions and properties as their mammalian counterparts (Kalueff, Stewart and Gerlai, 2014), gene duplication presents additional complexities when generating genetic models. For example, one of the two gene orthologues could trigger compensatory mechanisms when only one of them is edited. In other instances, only one of the zebrafish orthologues is homologous to the mammalian gene while some duplicated orthologues emerge new functions entirely different from their mammalian counterpart altogether. Together, these gene duplication event leads to socalled "sub-functionalization and neo-functionalization" problems (Spitsbergen and Kent, 2003; Lieschke and Currie, 2007). 


\subsection{STUDY AIMS}

The goal of this thesis was to clarify how dysfunction of proteins encoded by psychiatric risk genes contribute to disease. In particular, we investigated two genes (CACNA1C and CACNA1D) that encode L-type voltage-gated calcium channels (Cav1.2 and Cav1.3 respectively). We chose the aforementioned genes based on the high confidence of their reproducibility from various GWAS on their psychiatric risk association. Furthermore, seminal works from our collaborators found increased disease severity in patients and cardinal deficits from modelling data. We evaluated the in vivo functions of these genes using the zebrafish as a model organism. In order to arrive at the main goal, the following specific objectives were set:

1. Establish and validate a PPI assay platform for use towards behavioural characterisation of zebrafish mutants

2. Examine the effects of cacnalc mutations on zebrafish brain function

3. Examine the effect of cacnalda mutation on zebrafish brain function 


\subsection{MATERIALS AND METHODS}

\subsection{Zebrafish lines and husbandry}

In this thesis, we mainly used three zebrafish mutant lines, wild types of the strains $A B$ and Tupfel long-fin (TL). In paper II, we used TL wild type fish to validate the PPI protocol generated using two commercially available automated system and software. In paper III, we used the cacnalda splice variant mutant line (sa17298) to understand the effect of cacnalda mutations on zebrafish behaviour. Finally, in paper IV, we used AB wild type and two cacnalc mutant lines (sa10930 and sa15296) to investigate the role of cacnalc in zebrafish brain function. All the three mutant lines were generated by ENU mutagenesis by the Zebrafish Mutation Project (Sanger, UK), and obtained as fertilized embryos from the Zebrafish International Resource Center (Eugene, Oregon, USA) (Kettleborough et al., 2013).

We chose to focus on cacnalda and cacnalc because they have been replicated in numerous independent studies to be associated with psychiatric disorders. Both sa10930 and sa15296 are alleles of the cacnalc gene. The sa10930 mutant line carries a nonsense mutation in exon 6 , and in silico analysis predicts a premature stop codon effect (NM_131900.1: c.876T>A: p.Tyr292Stop) - exon 6 forms part of ion transport domain 1. On the other hand, the sal5296 mutant line carries a splice variant with the mutation positioned at the end of exon 35 but at the beginning of the next intron (NC_007115.7: g.155870T >C) - exon 35 forms part of ion transport domain 4. Finally, the sal7298 mutant line carries a point mutation in cacnalda, which spans an essential splice site (NC_007122.7: g.106573G >A).

Upon reaching adulthood, the fish were genotyped, and heterozygous animals outcrossed to $A B$ wild type (WT) zebrafish to get rid of potential off-target mutations. The fish were maintained at the Norwegian Centre for Molecular Medicine zebrafish facility under standard conditions (Aleström et al., 2019) 14-hour light and 10-hour dark cycle. Embryos were raised in an incubator at $28^{\circ} \mathrm{C}$ in embryo medium (1.5 mM HEPES, pH 7.6, $17.4 \mathrm{mM} \mathrm{NaCl}, 0.21 \mathrm{mM} \mathrm{KCl}, 0.12 \mathrm{mM}$ $\mathrm{MgSO} 4$, and $0.18 \mathrm{mM} \mathrm{Ca}(\mathrm{NO} 3)_{2}$ at $\mathrm{pH}$ 7.0). During the weekdays, fish were fed three times i.e., dry food (SDS) morning and evening, then Artemia Nauplii for the afternoon. Fish were fed only twice at weekends with dry food (SDS). Approval for experiments with animals was acquired per 
the Norwegian Animal Welfare Act (FOTS ID 15469 and 23935) and the European Convention for the Care and Use of Laboratory Animals (Directive 2010/63/EU).

\subsection{Primer design}

We used the Primer3 web tool to design all primers. Primer pairs meeting the following criteria were selected: size between 20-24 nucleotide, a GC content between 42-52\%, and temperature between $57-62{ }^{\circ} \mathrm{C}$. All primers were purchased from Sigma-Aldrich and used at $10 \mu \mathrm{M}$ final concentration.

\subsection{Genotyping}

Adult fish tail fin samples were collected under anesthesia and flash-frozen in liquid nitrogen for DNA extraction. Dream Taq Polymerase (\#EP0702, Thermofischer) was used for PCR with the following annealing temperatures: $\operatorname{sal} 10930\left(57^{\circ} \mathrm{C}\right)$, sal $5296\left(57.5^{\circ} \mathrm{C}\right)$, and $\operatorname{sal} 7298\left(59^{\circ} \mathrm{C}\right)$. The restriction enzymes, MseI (\#R0525M, New England BioLabs), HphI (\#ER1102, Thermo Fischer), and BstEII (\#R0162M, New England BioLabs) were used to digest the PCR products obtained from the sa10930 and sa15296, sa17298 lines respectively.

The following allelic-specific primers were used:

sa10930 forward 5'-ATGGTTCCCCTCCTTCAC-3'

sa10930 reverse 5'-AGTTCAAGGGAGAAGCAAAAG-3'

sa15296 forward 5'-AAGACTGTGGCAGTCACTTTG-3'

sal5296 reverse 5'-ACTGTACGGAGGGGGTAAAA-3'

sal7298 forward 5'-TGTGCTGGTGTTGTGTGTG-3'

sa17298 reverse 5'-TCAAGCCAGGAAGTACTGAAG-3' 


\subsection{Microscopy}

The Leica M205 FA stereomicroscope was used to take larval images in papers III and IV. The images were taken using the same magnification to make comparison easier.

\subsection{RT-qPCR}

To determine the effects of the mutations on gene expression, we used RT-qPCR to analyse the relative mRNA expression levels of cacnalda and cacnalc in papers III and IV respectively. Whole-larvae were snap-frozen in liquid nitrogen, total RNA isolated using the Invitrogen PureLink RNA Mini Kit (\#12183018A, Thermo Fischer). For quality control purposes, only samples that registered A260/280 and A230/280 nm ratios of $>1.8$ when tested with the Nanodrop 1000 spectrophotometer and displayed bands without smears in a $2 \%$ bleach agarose were used for analysis (Aranda, LaJoie and Jorcyk, 2012). RT-qPCR was performed using PowerUp Sybr green master mix with either the CFX384 Touch Real-Time PCR Detection System (1855485, Bio Rad) in papers III and IV or Applied Biosystems 7900HT Fast Real-Time PCR system (Applied Biosciences, Thermo Fischer) in paper IV.

\subsection{Preparation of samples for neurotransmitter measurement}

We used 1:1 ratio of acetonitrile and Millipore water to extract lysates from whole-larvae through sonication in order to investigate the levels of various neurotransmitters in mutants relative to controls in paper IV. After sonication, the samples were spun down through centrifugation and the supernatant collected into fresh tubes. The extracts were sent to Assoc. Prof. Wirgina Kuku-Koch at the Medical University of Lublin, Poland for analysis using a HPLC-ESI-Q-TOF-MS set-up.

\subsection{Drugs}

In paper II, larvae were pretreated in $10 \mathrm{mg} / \mathrm{ml}$ apomorphine for $10 \mathrm{~min}, 20 \mu \mathrm{M}$ haloperidol (HALO) for $20 \mathrm{~min}$, and $1 \mathrm{mM}$ ketamine for $10 \mathrm{~min}$. In paper III, larvae were pretreated for $24 \mathrm{hr}$ in either $5 \mu \mathrm{M}$ risperidone (RISP), $50 \mu \mathrm{M}$ HALO or $100 \mu \mathrm{M}$ valproic acid (VPA). Finally, in order 
to decrease the severity of oedema in the two cacnalc mutant lines (sa10930 and sa15290) used in paper IV, from $3 \mathrm{dpf}$ until the day of their use for experiments, larvae were exposed to $250 \mathrm{mM}$ mannitol (\#M4125, Sigma) as described previously (Hill et al., 2004). All inorganic compounds were dissolved in DMSO at a final concentration $\leq 0.5 \% \mathrm{v} / \mathrm{v}$ while organic compounds were dissolved in zebrafish E3 medium. All drugs were purchased from Sigma-Aldrich except for RISP, which was bought from TOCRIS, and VPA that was obtained from Sanofi Aventis.

\subsection{Behavioural assays}

Several comparative behavioural assays have been developed in the field of zebrafish neuroscience research, which along with the presence of several standardized and automated behavioural analysis system and tools, have made it possible to model relevant behavioural domains (Colwill and Creton, 2011). The following tests were used: prepulse inhibition, locomotor activity, lightdark transition, thigmotaxis and shoaling.

\subsubsection{Prepulse inhibition of the acoustic startle response}

In paper II, and parts of papers III and IV of this thesis, we used the automated live video tracking system called the ZebraBox Revo (ZebraLab, ViewPoint Life Sciences, France) and the automated video analysis software EthoVision version 14 (Noldus, Netherlands) to perform the PPI of the ASR test in larval zebrafish. The ZebraBox Revo uses a high-speed infrared camera that records videos at 1000 frames per second at $2048 \times 500$ resolution. The other components of the system include the following: a test cabinet, a stereo amplifier (Dynavox; CS-PA1 MK) and a dB meter (PCE instruments; PCE-MSM 4) to calibrate sound intensity, input and output cables, and the software (PPI generator) to generate and deliver acoustic stimuli. The ZebraBox Revo system allows for delivering acoustic stimulus with timing and waveforms generated by the PPI software. The intensity of the stimulus was calibrated and measured using the volume knob of the stereo amplifier and a decibel meter respectively. To determine the exact onset of stimuli, an infrared light source of $10000 \mathrm{~Hz}$ was coupled to the entire duration of the stimulus. The infrared light is not visible to the larvae; therefore, it does not interfere with the behaviour of the larvae. 
Next, we used EthoVision (Noldus, Netherlands), which is an automated tracking software to analyze the videos previously acquired using the ZebraBox Revo system. Tracking of the larvae was performed in a non-live mode with static subtraction of the background, which reduced artifacts. The tracked features were the center-point, nose-point, and tail-base of each larva. The absolute bend angle of the body was calculated for each larva using the tracked features. Various bend angle thresholds were set between $20-80^{\circ}$ averaged over 5 ms (i.e. 5-time frames). Once the tracking parameters were optimized, EthoVision software was used for the analysis of the larval response to the various acoustic stimuli. A change in body angle of $>25^{\circ}$ with a cut-off latency of $100 \mathrm{~ms}$ after stimulus onset was considered a positive response. The C-start threshold of $>25^{\circ}$ was determined after preliminary analysis of larvae not presented with any stimuli. To define the intensity of the startle stimulus, we presented $660 \mathrm{~Hz}$ tones of different stimulus intensities between $40-80 \mathrm{~dB}$ in increments of $5 \mathrm{~dB}$ and calculated using the following formula;

$$
\% \text { responders }=\frac{(\text { number of larvae responding })}{(\text { total number of larvae })} * 100
$$

Any stimulus intensity that was capable of eliciting a C-start response in $>70 \%$ of the larvae were considered a suitable startle stimulus as used in previous studies (Burgess and Granato, 2007b). We excluded larvae that responded $<60 \%$ to the startle stimulus. In all PPI experiments, a pseudorandom order was used to interleave prepulse trials with pulse alone trials. Where multiple stimuli were presented in an experiment, an inter-trial interval (ITI) of $30 \mathrm{sec}$ was used. We calculated \% PPI using the formula below:

$\% \mathrm{PPI}=\frac{\text { (percentage responding to startle stimulus) }- \text { (percentage responding to prepulse }+ \text { startle stimulus) }}{\text { (percentage responding to startle stimulus) }} * 100$

All PPI experiments were performed on individual larvae at $6 \mathrm{dpf}$, in a custom-made plexiglass plate of 96-well format. We used the plexiglass plate to reduce interference from shadows that we observed initially when using commercial plates. Larvae were placed individually in a well (16 larvae per plate in total) and acclimated to a 100 Lux illuminated ZebraBox Revo for 5 minutes before the experiments were started. Burgess \& Granato (2007) reported an influence on the startle response by temperature. Hence, we performed all experiments in a temperature-controlled setting 
$\left(27 \pm 1^{\circ} \mathrm{C}\right)$. To reduce the background white noise of the room, the ZebraBox Revo was insulated with a custom-built sound booth. In all experiments, we used a $100 \mathrm{~ms}$ startle stimulus (pulse) of $660 \mathrm{~Hz}$, and all prepulse stimuli were given for $5 \mathrm{~ms}$ at $440 \mathrm{~Hz}$.

\subsubsection{Locomotor-based analysis}

In papers III and IV of this thesis, we used the automated live video tracking system called the ZebraBox (ZebraLab, ViewPoint Life Sciences, France) to perform the following locomotor based tests in larval zebrafish: locomotor activity, light-dark transition, thigmotaxis, and shoaling. The ZebraBox system consists of an infrared light source, a high-resolution digital video camera to capture larval movements within a defined period, and the ZebraLab software to analyze larval locomotor activity (Colwill and Creton, 2011). For all experiments, 6 dpf larvae were used except in the shoaling tests where we used $7 \mathrm{dpf}$ larvae. On the day of the experiments, the larvae were transported from the incubator, allowed to acclimate to the conditions of the recording room for a minimum of one hour. All experiments were performed between 10:00 and 18:00. We used custom-made 48 well plates made from plexiglass for the locomotor activity and light-dark transition tests. A 24 well plate (\#83.3922.500, Sarstedt, Germany) and a round dish [of diameter $60 \mathrm{~mm}$, height $15 \mathrm{~mm}$, and volume $30 \mathrm{ml}$ (\#82.1194.500, Sarstedt)] were used for the thigmotaxis and shoaling tests respectively. In paper IV, we did not use homozygous mutants in the thigmotaxis and shoaling tests because the reliability of the tests depend on the mobility of the animals. However, cacnalc homozygous mutants have reduced mobility.

\subsubsection{Locomotor activity test}

Habituation and tracking of larvae were performed under dark conditions for 15 and 10 min respectively. The total movement was defined as the average of the sum of large + small movements expressed in the $\mathrm{mm}$. 


\subsubsection{Light-dark transition test}

Larvae were first habituated in the dark chamber for $15 \mathrm{~min}$, then the light switched on to begin tracking for $10 \mathrm{~min}$ followed by a light switch off (darkness) for another $10 \mathrm{~min}$ of tracking. Total movement in each illumination state was calculated as the average of the sum of large + small movements expressed in the mm per time bin.

\subsubsection{Thigmotaxis test}

Each well was divided into an outer and inner zone and thigmotaxis was calculated as the $\%$ total distance moved in the outer zone as described by (Schnörr et al., 2012; Liu et al., 2016). The preference of each fish to remain at the periphery or explore the center of the arena was monitored for 10 min spontaneous behaviour in either light or dark conditions.

Thigmotaxis $(\%$ distance in outer zone $)=\frac{(\text { Distance outer zone })}{(\text { Distance outer }+ \text { inner zone })} * 100$

\subsubsection{Shoaling test}

Larvae (5 larvae/arena) were placed in the center of the testing arena. The testing arena was then placed in the dark ZebraBox. After 15 minutes of habituation in the dark, a 20-minute recording session was performed also in the dark. The unit of measurement in this case is a shoal made up of 5 larvae. Except for homozygous mutants, it was difficult to sort WT and heterozygous mutant larvae without genotyping and since genotyping larvae would result in either the injury or death of larvae we had to come out with a testing paradigm described previously (Maaswinkel, Zhu and Weng, 2013). Whereas the control group comprised WT siblings, the mutant group was typically a mixed population of heterozygous and wild type siblings of the same line. This means that there was variation in the ratio of heterozygous to WT in each independent shoaling population. The shoaling characteristics we measured were nearest neighbor distance (NND), and inter-individual distance (IID) as described elsewhere (Miller and Gerlai, 2012). 


\subsection{Local field potential recordings}

7-dpf larvae were prepared and handed over to two post-doctoral researchers in the Esguerra lab (Dr. Kinga Gawel and Dr. Wietske van der Ent) for electrode local field potential recordings (LFP) from the optic tecta of larvae as previously described (Gawel, Kukula-Koch, et al., 2020; Gawel, Turski, et al., 2020; Tiraboschi et al., 2020). The aforementioned researchers performed the analysis of seizure-like activity. Afterwards, the LFP recordings were sent to Dr. Tuomo MäkiMarttunen for spectral power analysis. 


\subsection{SUMMARY OF RESULTS}

\section{Paper I: A critical review of zebrafish schizophrenia models: Time for validation?}

The zebrafish has emerged as a valuable and attractive model for various psychiatric disorders including schizophrenia. In this review, we discussed the advantages of using zebrafish as a model of psychiatric disorders and the relevance of zebrafish larvae in schizophrenia studies. We further discussed the available pharmacological and genetic models of schizophrenia in the zebrafish. Throughout, we presented perspectives into some limitations and the way forward.

\section{Paper II: Pharmacological validation of the prepulse inhibition of startle response in larval zebrafish using a commercial automated system and software.}

Prepulse inhibition is a form of sensorimotor gating in which the startle response is attenuated when a weak non-startling stimulus is presented shortly before the startling stimulus (Bhandiwad et al., 2013). Deficits in prepulse inhibition are observed in schizophrenia patients and animal models (Burgess and Granato, 2007b; van den Buuse, 2010; Bhandiwad et al., 2013) making it a useful endophenotype for the genetic analysis of diseases such as schizophrenia (van den Buuse, 2010). Even though prepulse inhibition in humans and rodents are performed with standardized automated systems, in the zebrafish research community, many labs have to rely on developing custom-made systems to perform the test. This presents challenges for achieving data reproducibility and replicability with regard to standardisation (Gerlai, 2019).

In this paper, we combined two commercially available systems ZebraBox Revo system with the EthoVision software, to generate a protocol for the performance and analysis of prepulse inhibition response in zebrafish larvae. Following published work, we validated the protocol using three pharmacological modulators of the prepulse inhibition response i.e., apomorphine, haloperidol, and ketamine. The data captured by the ZebraBox Revo and analyzed with EthoVision suggests that these commercially available systems provide a validated platform for performing the prepulse inhibition assay and enables reliable detection of pharmacologically induced changes in the prepulse inhibition response. 


\section{Paper III: Zebrafish larvae carrying a splice variant mutation in cacnald: a new model for schizophrenia-like behaviours?}

Persons with variants in $C A C N A 1 D$ have increased risk of developing psychiatric disorders such as bipolar, schizophrenia and autism spectrum. Our understanding of the functional effects of these variants are thus crucial in understanding disease mechanisms. In this study, we describe the gene expression, electroencephalographic (EEG) characteristics, neurobehavioural changes and pharmacological modulation of the observed behaviours in zebrafish larvae carrying a point mutation in the essential splice site of the cacnalda gene (sa17298) (NC_007122.7: g.106573G >A). We used four locomotor-associated assays (spontaneous locomotor activity, lightdark test, thigmotaxis and startle response to dark flashes) and prepulse inhibition of the acoustic startle response which have been used to model aspects of various brain disorders in larval and adult zebrafish.

In heterozygotes, sa17298 resulted in haploinsufficiency evidenced by the reduction of mRNA expression of transcript variants 201 and 202 by 50\%, while in the homozygotes, we observed a putative gain of function - i.e., increased levels of transcript variant 201 compared to wild type. Although no morphological abnormalities were detected in the mutants (heterozygotes and homozygotes), homozygotes had low viability. Hence, all behavioural tests were performed in only heterozygotes. Behaviourally, we observed profound alterations in the response of heterozygous sal 7298 mutants when tested in the locomotor activity assay under constant light conditions, during light-dark transition and in the startle response assay (using dark flashes). However, we did not observe thigmotaxis-related and prepulse inhibition abnormalities. Exposure of larvae to the antipsychotics, risperidone ( $5 \mu \mathrm{M}, 24 \mathrm{hr})$ and haloperidol $(50 \mu \mathrm{M}, 2 \mathrm{hr})$ completely reversed cacnalda mutation-induced hyperactivity to wild-type control levels while valproate $(100 \mu \mathrm{M}, 2$ hr) significantly decreased, but did not reverse hyperactivity. Furthermore, we found no significant difference in seizure-like discharges in mutants when compared to wild type controls, indicating that perhaps the hyperlocomotor activity observed is not due to convulsive-like activity. Together, these findings demonstrate that mutations in the cacnalda gene can induce schizophrenia-like behaviours in larval zebrafish. 


\section{Paper IV: Functional characterisation of single nucleotide variants (SNVs) in the zebrafish neuropsychiatric risk gene cacnalc.}

Although GWAS has identified several single nucleotide polymorphisms in CACNAIC that confer greater risk for several neuropsychiatric disorders, the consequences of these variants in relation to brain function is poorly understood. We evaluated how different mutations in the same gene (i.e., cacnalc) affect brain function with the aim of enhancing our understanding of disease pathogenesis. Two zebrafish lines with mutations in cacnalc that were generated by ENU mutagenesis were used for this study. The first line, sa10930, carries a specific thymidine $(\mathrm{T})$ to adenosine (A) point mutation $(\mathrm{T}>\mathrm{A})$ in exon 6 resulting in a premature stop codon (TAA) at amino acid 292 (NM_131900.1: c.876T>A: p.Tyr292Stop). The second line, sa15296, carries a point mutation that results in a thymidine $(\mathrm{T})$ to cytosine $(\mathrm{C})(\mathrm{T}>\mathrm{C})$ substitution in the donor splice site of intron 35-36, giving rise to essential splice variant (NC_007115.7: g.155870T >C).

We performed phenotypic analysis of the two mutant lines at the molecular, behavioural, electrophysiological and neurochemical levels. We found both distinct and common defects in these two mutant models. Homozygous mutants of both lines displayed pericardial oedema, craniofacial abnormalities and were embryonic lethal consistent with data from other vertebrate studies. Molecular analysis revealed significant reduction in the mRNA levels of cacnalc in homozygotes of both mutants pointing to a loss of function effect.

Behaviourally, we found that both mutant lines showed hypolocomotor activity, insensitivity to light and dark stimuli and reduced prepulse inhibition in the homozygous larvae while the heterozygotes of both mutants displayed shoaling deficits as observed in the increased interindividual distance between mutant larvae. In contrast, locomotor activity and zone preference of heterozygous sa10930 mutants were comparable to controls, whereas heterozygous sa15296 mutants displayed hypolocomotor activity and showed a preference for the inner zone of the open field when compared to controls. Although heterozygotes of both mutants were responsive to light/dark sensory stimuli, heterozygotes carrying the sa10930 mutation showed comparable and significantly reduced locomotor activity in the light and dark phases respectively, whereas the opposite was observed in the case of heterozygotes carrying the sa15296 mutation. 
Electrophysiological analysis revealed that homozygous mutants of both lines had significantly lower spectral power in the low delta-frequency $(0.5-2 \mathrm{~Hz})$ range than the control larvae. Conversely, homozygous mutants of the two lines only showed sporadic differences in the beta and gamma frequency ranges. The spectral slopes, determined for frequency range $50-200 \mathrm{~Hz}$, were significantly flatter in the homozygous mutants than in the control larvae, suggesting an increased excitability in the neural circuits of the mutant fish. Furthermore, we found that homozygotes of the non-coding region mutant sa15296, but not with the coding region mutant sal0930 discharged seizure-like activity in their EEG.

Furthermore, neurochemical studies revealed imbalances in the levels of major neurotransmitters in the two mutant lines when compared to controls. We found enhanced dopamine and serotonin, decreased GABA and unaffected levels of glutamate in homozygous sa10930. In the homozygous sa15296 larvae, increased serotonin and glutamate levels were observed, whereas levels of GABA and dopamine were unaltered. The GLUT/GABA ratio was increased in both sa10930 and sa15296 mutant lines, further suggesting overall increased excitability.

Finally, we assessed whether selected downstream pathways were affected by mutations in the cacnalc. We found a statistically significant reduction in the mRNA levels of bdnf but no significant changes in c-fos levels in sa10930 mutants, whereas in the sa15296 mutant, the mRNA levels of both $b d n f$ and $c$-fos were significantly reduced relative to control larvae.

Overall, our study provides further information on how different mutations of the cacnalc gene may lead to changes in gene expression, electrophysiological and neurochemical properties and behavioural abnormalities relevant to psychiatric disorders. 


\subsection{GENERAL DISCUSSION}

As more and more susceptibility genes implicated in PDs continue to be identified by GWAS, (Cross-Disorder Group of the Psychiatric Genomics Consortium, 2013; Ripke et al., 2014; Pardiñas et al., 2018; Mullins et al., 2021) it has become increasingly important to unravel the role these genes play in the development of disease. This has also provided opportunities for new genetic models and phenotypic drug screens. The L-type calcium channel genes, CACNA1C and $C A C N A 1 D$ have been associated with PDs, with specific variants suggested to present an increased risk for BP, SCZ, ASD and many other disorders (Cross-Disorder Group of the Psychiatric Genomics Consortium, 2013; Scholl et al., 2013; Ripke et al., 2014; Pinggera et al., 2015, 2017; Ross et al., 2016; Hofer et al., 2020). Calcium signalling is known to play a vital role in synaptic function, learning and memory, and the balance between neuronal excitation and inhibition (Devor et al., 2017). This thesis aimed to examine the roles of cacnalc and cacnalda in zebrafish brain function. In understanding the changes that occur because of cacnalc and cacnalda aberrations, we hope to link genetic risk for psychiatric disorders with specific symptoms of illness rather than disease categories.

The first specific aim of this thesis was to set-up and validate a PPI test platform to be used in characterising zebrafish mutants. To achieve this, we first investigated the ability of two commercially available automated systems (ViewPoint Revo and EthoVision) to deliver acoustic startle stimuli and measure the ASR by generating a protocol that utilises the two systems. Next, we validated the ability to attenuate startle response when a prepulse stimulus is presented. Furthermore, we explored the validity of the protocol by using pharmacologic agents that either attenuate or augment the zebrafish PPI (Paper II). The second specific aim of this thesis was to characterise a zebrafish line with a mutation in the psychiatric risk gene, cacnalda. We examined the gene expression, behavioural, and electrophysiological consequences of this mutation (Paper III). We then investigated whether the antipsychotics haloperidol and risperidol and the mood stabilizer valproate could ameliorate some of the behavioural deficits we observed. Similarly, the third specific aim of this thesis was to characterise two zebrafish lines carrying a mutation in the psychiatric risk gene, cacnalc. We examined the molecular, behavioural, electrophysiological and neurochemical effects of mutations in cacnalc (Paper IV) and whether this would result in dysfunctions reminiscent of endophenotypic domains relevant to psychiatric disease. The 
following sections will review each experimental result in relation to these three specific aims, and the future directions for research are discussed.

\subsection{Set-up and validation of a PPI test platform}

In order to perform initial characterization of sensorimotor behaviour displayed by mutant zebrafish, it was necessary to optimize and validate the PPI test and test the reliability of the measurements thereof (assay robustness). Using the recently launched ZebraBox Revo system (ViewPoint, France), in combination with the EthoVision software (Noldus, Netherlands), Paper II showed that the ZebraBox Revo system could deliver acoustic startling stimulus capable of eliciting an ASR from $6 \mathrm{dpf}$ larvae and that the ASR could be measured using the EthoVision software. Specifically, we were able to replicate the results of apomorphine, haloperidol and ketamine on the PPI response of larvae. The data captured by the ZebraBox Revo and analyzed with EthoVision suggests that these commercially available systems provide a validated platform for performing the PPI assay that enables reliable detection of pharmacologically induced changes in the PPI response. Although both systems are commercially available for performing and analysing a repertoire of zebrafish behaviour, this is the first time they have been used for performing the PPI test. PPI deficit is a robust phenotype in SCZ and has been studied extensively in animal models (Gould and Gottesman, 2006; van den Buuse, 2010; Thyme et al., 2019).

Our results demonstrate the capability of the combined ZebraBox Revo + EthoVision set-up to carry out the prepulse inhibition test in zebrafish larvae. Thus, these findings will help to standardise protocols used in future zebrafish behavioural assays. The protocol and findings of Paper II are consistent with literature (Burgess and Granato, 2007b) and was useful in designing a testing paradigm for assessing the impact of mutations on sensorimotor gating in three larval lines.

\subsection{Morphological consequences of mutations in LTCCs}

As Cav1.2 and Cav1.3 channels are also expressed in many tissues outside the brain (Heyes et al., 2015; Kabir, Martínez-Rivera and Rajadhyaksha, 2017; Andrade et al., 2019), it is possible that mutations causing aberrant gene function are likely to be associated with other (syndromic) organ 
dysfunctions especially cardiac defects.

We observed no morphological abnormalities in cacnalda mutants (heterozygotes and homozygotes), although homozygote mutants had low viability. Because Cav1.3 are expressed at ribbon synapses of cochlear hair cells, mice, human patients and zebrafish with null mutations have hearing impediments (Platzer et al., 2000; Sidi et al., 2004; Baig et al., 2011). In fact, previous studies have shown that zebrafish with cacnalda nonsense mutations swim in circles because they have balance defects (Sidi et al., 2004). The absence of a morphologic phenotype in the cacnalda line may be related to the nature of the mutation in our fish - splice variant mutation as against the nonsense mutant previously described. With the exception of hearing defects, no morphologic impairments have been reported in mutant mice.

On the other hand, homozygous mutants of both cacnalc lines (sa10930 and sa15290) had pericardial oedema, craniofacial abnormalities and were embryonic lethal consistent with data from previous studies in zebrafish (Rottbauer et al., 2001; Thyme et al., 2019). Similar to our mutants, constitutive Cav1.2 homozygous knockout mice are embryonic lethal (Seisenberger et al., 2000; Thyme et al., 2019). Although Cav1.2 heterozygous mice have a cardiac phenotype, which is exacerbated by stress (Goonasekera et al., 2012), we did not observe any differences between heterozygous cacnalc mutants and their WT siblings. It is worth mentioning that humans with GOF mutations in CACNAIC (Timothy syndrome) also have cardiac phenotype, craniofacial dysmorphologies, and webbing of the fingers and toes (Splawski et al., 2004, 2005). Taken together, the morphologic data of all three mutants are consistent with previous studies.

\subsection{Mutations in LTCCs lead to changes in gene expression}

Since calcium is a critical second messenger in cells (e.g., neurons), it is likely that mutations in $C A C N A 1 C$ can induce changes in either the gating or expression of the channel, which may lead to reduced or increased calcium entry during neuronal electrical activity. To determine the molecular consequences of the mutations, we performed RT-qPCR to assess basal mRNA levels. In humans as well as rodents, neuronal LTCCs (CACNA1D and CACNA1C) undergo alternative splicing thereby generating several splice variants (Bhat et al., 2012; Lipscombe and Andrade, 2015). In the zebrafish, two reported protein coding transcript variants of both cacnalda and 
cacnalc exist (see ensembl.org). In both instances, the major difference between the two variants is an additional exon in transcript variant (tv) 201 and 202 of the cacnalda and cacnalc respectively (see ensembl.org).

Unlike $C A C N A 1 C$, no study to the best of our knowledge has assessed the relative expression of $C A C N A 1 D$ in humans carrying susceptibility variants or in genetic/pharmacologic models. However, using electrophysiological techniques i.e. patch clamping, some CACNA1D mutations identified in humans have been shown to cause hypersensitivity of the Cav1.3 channel and thus, those mutations are predicted to be GOF (Pinggera and Striessnig, 2016). Recent studies have found mutations in the CACNA1D that lead to hypersensitization of Cav1.3 suggesting a GOF using electrophysiological measurements of calcium currents (Pinggera et al., 2015).

The cacnalda line, sal 7298 harbours a missense mutation in the intronic region specifically at the donor splice site between intron 20-21 of the cacnalda transcript variant 202 (see zfin.org). Because transcript variants tend to be expressed differently across tissue and time (developmental stages) (Lipscombe and Andrade, 2015; Pinggera and Striessnig, 2016), we designed three pairs of primers that would recognize both $t v 201$ and $t v 202$ (total cacnalda), tv201 only and $t v 202$ only. RT-qPCR analysis revealed that heterozygous sa17298 mutants had about $50 \%$ reduction in total cacnalda, tv202 and tv201 mRNA levels mimicking haploinsufficiency effects. Interestingly, in homozygous sal 7298 mutants, the mRNA levels of total cacnalda and $t v 202$ showed 60 and $90 \%$ reduction respectively. However, a $50 \%$ increase in $t v 201$ was observed suggesting a transcript specific GOF effect.

Two mutant lines of cacnalc were used for this thesis, sa10930 and sa15296. RT-qPCR analysis revealed over $90 \%$ reduction in the total cacnalc mRNA levels in homozygous mutants of both sal0930 and sa15296. The sa10930 mutation occurs in the exon 6 which encodes the TMD1. Since this region is responsible for the sensitivity of the $\mathrm{Ca}_{\vee} 1.2$ channel, we predicted that the mutation likely decreases the activation of the channel, which may perturb downstream pathways as well as functions directly dependent on the proper functioning of $\mathrm{Ca}_{\mathrm{v}} 1.2$. This predicted pathogenic effect of the sa10930 mutation happens because of the LOF of Cav1.2 channel. The aforementioned hypothesis seems likely because RT-qPCR showed decreased levels of cacnalc mRNA when compared to controls.

Similar to the sal 7298 mutation in cacnalda, the sal 5296 mutation also occurs at the donor splice 
site. Splice site mutations could lead to the creation of a splice variant via exon skipping or influence the regulation of gene expression. The former i.e., exon skipping, was not observed but RT-qPCR analysis revealed the latter to be the case - i.e., decreased cacnalc mRNA levels. The gene-disrupting effects of splice site mutations occurs when cryptic splice sites are used (Abramowicz and Gos, 2018). If the cryptic splicing produces an mRNA with a premature termination codon, the mRNA becomes unstable and may be subjected to nonsense-mediated mRNA decay, which could lead to no or reduced mRNA translation and hence, a decrease in protein levels (Abramowicz and Gos, 2018). The decreased gene expression observed in the splice site mutants may be similar to that reported for SNP rs100637 (a variant in intron 3 of CACNA1C) which resulted in altered $C A C N A 1 C$ gene expression levels in both human carriers (Bigos et al., 2010; Gershon et al., 2014), and induced human neurons (Yoshimizu et al., 2015). The reported expression changes were region specific in that in some areas, an increase was observed, while in others, a decrease was reported - thus, underscoring the complexity of gene transcription.

\subsection{Altered LTCC function is associated with behavioural deficits}

As mentioned earlier, LTCCs contribute to the proper functioning of the brain circuitry involving the mesolimbic system (which involves the circuitry between the VTA and NAc, with modulatory inputs from the hippocampus, medial prefrontal cortex, and amygdala), hippocampus, and amygdala, which are implicated in the pathophysiology of various psychiatric disorders (Bhat et al., 2012; Zamponi, 2016). Therefore, it is anticipated that dysregulation in LTCC function could affect behaviours controlled by the brain circuits they modulate. Furthermore, SCZ and autism patients are reported to have social, cognitive and behavioural deficits (Patel et al., 2014; Vorstman and Burbach, 2014). Persons with GOF and LOF mutation in Cav1.2 and Cav1.3 present with psychiatric abnormalities (Bhat et al., 2012; Pinggera and Striessnig, 2016). Similarly, broader phenotypes such as deficits in PPI, latent inhibition and sleep disturbance are present in some Cav1.2 SNP carriers that is reminiscent of aspects of psychiatric disorders and/or traits (Harrison, 2015; Andrade et al., 2019). We performed different behavioural assays such as locomotor, thigmotaxis and light/dark transition tests, shoaling test, startle response to dark flashes and PPI of the acoustic startle stimulus tests, which measure motor function, stress/anxiety, social behaviour, non-associative learning test and sensorimotor gating respectively. These behavioural 
tests are endophenotypically relevant in modelling various aspect of psychiatric disorders and are thus useful for characterising our mutants (Gould and Gottesman, 2006; Kalueff, Stewart and Gerlai, 2014; Stewart et al., 2014; Haesemeyer and Schier, 2015; Sakai, Ijaz and Hoffman, 2018).

Behaviourally, we observed profound alterations in the response of heterozygous sa17298 mutants when tested in the locomotor activity assay under constant light conditions, during light-dark transition and in the startle response assay (using dark flashes). However, we did not observe thigmotaxis-related and PPI abnormalities. Exposure of larvae to the antipsychotics, risperidone (5 $\mu \mathrm{M}, 24 \mathrm{hr}$ ) and haloperidol ( $50 \mu \mathrm{M}, 2 \mathrm{hr}$ ) completely reversed cacnalda-induced hyperactivity to WT control levels while valproate (100 $\mu \mathrm{M}, 2 \mathrm{hr}$ ) significantly decreased, but did not reverse hyperactivity.

Analysis of the two-cacnalc mutant lines (sa10930 and sa15296) revealed a number of unique as well as overlapping behavioural phenotypes. We found that both cacnalc mutant lines showed hypolocomotor activity, insensitivity to the light/dark sensory stimuli and reduced PPI in the homozygous larvae while the heterozygotes of both mutants displayed shoaling deficits as seen in the increased IID. However, the locomotor activity and zone preference of heterozygous sa10930 mutants were comparable to WT whereas heterozygous sal5296 mutants had hypolocomotor activity and showed a preference to the inner zone of the open field when compared to WT. Although heterozygotes of both mutants were responsive to light/dark sensory stimuli, heterozygotes with the sa10930 mutation showed comparable and significantly reduced locomotor activity in the light and dark phases respectively whereas the opposite was the case in the heterozygotes with the sal5296 mutation. Using rodent models, diverse mutations in Cacnalc have been generated to study its behavioural roles (Moon et al., 2018). Indeed, impairments in motor function such as hypoactivity, social interactions, cognitive functions, fear, and anxiety have been reported in some Cacnalc mutant lines (Moon et al., 2018). Mice with conditional knockout of forebrain Cav1.2 displayed unusually high anxiety-like behaviour (Dao et al., 2010; Lee et al., 2012), with no change in corticosterone levels compared with control littermates (Lee et al., 2016). Heterozygous mice with Cacnalc deletion showed hypoactivity, a general anxiety-like behaviour triggered by external stimuli which pervaded social interactions, differences in exploratory patterns of Cacnalc mutant mice suggestive of a behavioural effect in a social setting (Kabitzke et al., 2018). In zebrafish, LOF cacnalc mutants generated via CRISPR/Cas9 were found to 
exhibit a plethora of phenotypes - heterozygote mutants exhibited increased forebrain activity while homozygous mutants at $4 \mathrm{dpf}$ displayed abnormal startle magnitude, PPI response, light/dark activity and swimming behaviour (Thyme et al., 2019).

Locomotor and locomotor-associated behaviours such as the light-dark test, startle response and thigmotaxis have been used to model aspects of various brain disorders in larval and adult zebrafish (Kalueff, Stewart and Gerlai, 2014; Sakai, Ijaz and Hoffman, 2018; Basnet et al., 2019). For example, larval locomotor activity is typically measured as the distance moved (Burgess and Granato, 2007a). Hypolocomotion is often associated with sedation, motor defects such as akinesia (Kalueff et al., 2013) or models of ASD (Sakai, Ijaz and Hoffman, 2018). On the other hand, hyperlocomotion is typically associated with epilepsy (Afrikanova et al., 2013; Baraban, Dinday and Hortopan, 2013; Kalueff et al., 2013; Gawel, Kukula-Koch, et al., 2020; Tiraboschi et al., 2020), anxiety (Maximino et al., 2010; Kalueff et al., 2013) and positive symptoms of SCZ (Seibt et al., 2010, 2011; Kalueff et al., 2013). Exaggerated reactivity in the light-dark test has been used to model anxiety (Stewart et al., 2014). Thigmotaxis refers to the preference of an animal to stay at the periphery while avoiding the centre of an open field (Schnörr et al., 2012). This behaviour is mostly used as an indication of anxiety and fear (Maximino et al., 2010; Kalueff et al., 2013; Stewart et al., 2014) as well as a behaviour associated with ASD (Sakai, Ijaz and Hoffman, 2018). Zebrafish startle in response to unexpected visual, acoustic, and tactile stimuli, typically as an innate reflex to avoid danger (Norton, 2012; Kalueff et al., 2013). However, when exposed to the same stimulus multiple times, zebrafish can habituate to the stimulus, resulting in a decrease in rapid movements (Norton, 2012). Thus, startle response tests are useful in studying escape and avoidance behaviours in the context of fear and anxiety in zebrafish larvae (Colwill and Creton, 2011).

\subsection{Altered LTCC function causes changes in neural oscillations and neurotransmitter levels}

\subsubsection{Seizure-like discharges}

In zebrafish, LFP recordings equivalent to EEG has routinely been used to measure neuronal activity changes regarding seizure-like discharges (Baraban et al., 2005; Afrikanova et al., 2013). 
For this thesis, resting state LFP recordings were obtained from the optic tecta of larvae aged $7 \mathrm{dpf}$ over a 20 min recording period. Analysis of the LFP data of the cacnalda (sa17298) mutants revealed no significant differences in the seizure-like discharges between mutants (hetero and homozygous) and their WT controls. For the cacnalc mutant lines, we found that homozygotes of the non-coding region mutant sa15296, but not with the coding region mutant sa10930 discharged seizure-like activity in their LFP traces.

Although hyperlocomotor activity is a surrogate readout of seizure-like behaviour in zebrafish (validated usually with LFP recordings) (Afrikanova et al., 2013; Baraban, Dinday and Hortopan, 2013), a recent study found that larvae with seizure-like discharges were hypoactive in the locomotor test (Hotz et al., 2021). Suggesting that the type of seizures may inform the behavioural readout i.e. tonic-clonic seizures manifest as hyperlocomotor activity while absence seizures may manifest as hypolocomotor activity. In addition, hyperlocomotion is sometimes used an endophenotype of psychosis in animal models (Seibt et al., 2010, 2011; de Bartolomeis et al., 2013; Kalueff, Stewart and Gerlai, 2014; Langova et al., 2020). Although the sa15296 mutants were hypoactive, the LFP traces suggested they had seizure-like activity. Interestingly, the LFP traces did not show the classical high amplitude and long duration peaks of the seizure-like patterns observed in pharmaco-induced seizures (Orellana-Paucar et al., 2012; Afrikanova et al., 2013; Gawel, Kukula-Koch, et al., 2020), or the genetic Dravet model (Baraban, Dinday and Hortopan, 2013; Tiraboschi et al., 2020). Therefore, it is possible that the discharges observed could be something other than seizures. The aforementioned can be confirmed by testing the efficacy of different anti-seizure drugs in abolishing the discharges.

Moreover, most of the mutations associated with seizures are those with GOF effects in CACNA1C (Splawski et al., 2004; Boczek et al., 2015; Bozarth et al., 2018), and CACNA1D (Klassen et al., 2011; Scholl et al., 2013; Pinggera et al., 2017) leading to hypersensitive channels. In fact, activation of LTCCs in mice with BayK8644 caused convulsive behaviour (Littleton, Little and Whittington, 1990). However, blockage of LTCCs with nimodipine and nifedipine decreased seizures (N'Gouemo et al., 2015) while nimodipine, nitrendipine and PN 200-110 blocked convulsive behaviour associated with chronic alcohol withdrawal (Littleton, Little and Whittington, 1990). Thus, in agreement with a previous study that suggests that increased LTCC channel current (patch clamp) is associated with susceptibility to seizures induced by alcohol 
withdrawal (N'Gouemo and Morad, 2003). To understand the molecular correlate of the increased LTCC channel currents, N'Gouemo and colleagues reported the upregulation of Cav1.3 at both the mRNA and protein levels while Cav1.2 was only upregulated at the mRNA level (N'Gouemo et al., 2015). Thus, it is not surprising that no significant seizure-like activity in cacnalda (sa17298) and cacnalc (sa10930) or classical seizure-like discharges in cacnalc (sa15296) were observed.

\subsubsection{Spectral analyses}

Spectral analyses of the EEG recordings of the cacnalc mutant lines (sa10930 and sa15296) revealed that homozygous mutants of both lines had significantly lower spectral power in the low delta-frequency $(0.5-2 \mathrm{~Hz})$ range than the WT control. Additionally, homozygous mutants of the two lines only showed sporadic differences in the beta $(13-30 \mathrm{~Hz})$ and gamma $(30-100 \mathrm{~Hz})$ frequency ranges. The spectral slopes, determined for the frequency range 50-200 Hz, were significantly flatter in both of the cacnalc homozygous mutants than in the WT larvae, suggesting an increased excitability in the neural circuits of the mutant fish.

Cortical networks engage in oscillatory activity at several frequency bands: delta $(1-4 \mathrm{~Hz})$, theta $(4-8 \mathrm{~Hz})$, alpha $(8-13 \mathrm{~Hz})$, beta $(13-30 \mathrm{~Hz})$, and gamma $(30-200 \mathrm{~Hz})($ Uhlhaas et al., 2008). There is extensive evidence to their roles in normal brain functioning, and their association with several cognitive processes (Uhlhaas et al., 2008). Expectedly, changes in oscillations are correlated with cognitive dysfunction and some symptoms in various neurological and psychiatric disorders (Uhlhaas et al., 2008; Uhlhaas and Singer, 2010). Insights into the mechanisms underlying the generation and synchronization of cortical oscillations reveal its dependence on E/I balance (Uhlhaas et al., 2008; Uhlhaas and Singer, 2010; Plumbly et al., 2019) - this is particularly true for beta/gamma oscillations (Uhlhaas and Singer, 2013). Altered synaptic E/I balance is one of the theories proposed as a mechanism underlying PDs (Pocklington et al., 2015). In a study by Gao et al (2017), they inferred synaptic E/I balance from field recordings using a computational model made up of AMPA and $\mathrm{GABA}_{\mathrm{A}}$ currents where they showed that the E/I ratio affected the slope characteristics i.e. 1:6 ratio when compared with a 1:2 ratio had steeper (more negative) slope. To validate the findings, they used hippocampal LFP recordings from the stratum pyramidale of CA1 of actively foraging rats. Analysis of the rat data showed that the slope of 
regions of the CA1 with more excitation were flatter (more positive) while the slope of regions with more inhibition were steeper. Furthermore, they used multi-electrode electrocorticograph of macaques in non-treated awake state and propofol anaesthetised state. Analysis of the data showed that the slope of macaques in the awake state was more positive, an indication of arousal/excitation while that of the anaesthetised state was steeper consistent with the sedative/inhibition effects of propofol (Gao, Peterson and Voytek, 2017).

A role of LTCCs on E/I balance have been reported using models with LTCCs hypofunction (Kabir et al., 2017; Kabir, Martínez-Rivera and Rajadhyaksha, 2017; Plumbly et al., 2019). Blockage of LTCCs in primary culture of mice forebrain cortical neurons with nifedipine (for 24-hr treatment) increased the amplitude and frequency of miniature excitatory postsynaptic currents (Gong et al., 2007). Similarly, blockage of LTCCs in cultured hippocampal neurons with nifedipine decreased the number of $\mathrm{GABA}_{\mathrm{A}} \mathrm{Rs}$ at synaptic sites, and decreased the amplitude but not the frequency of miniature inhibitory postsynaptic currents or expression of the glutamate decarboxylase 65 (GAD65) (Saliba et al., 2009). Furthermore, in mice harboring loss of Cacnalc in excitatory glutamatergic neurons of the forebrain ( $\mathrm{fbKO}$ ), a higher E/I ratio in layer 5 pyramidal neurons was revealed as a result of an increase in the amplitude and frequency of miniature excitatory postsynaptic currents in layer-5 neurons of the prefrontal cortex (Kabir et al., 2017). Indeed, blockage of LTCCs in human induced pluripotent stem cells (hiPSCs) derived neuronal cultures with Diltiazem and Nifedipin (LTCC antagonists) caused a dose-dependent reduction of the synchronised burst intervals albeit with little effects to the basal excitatory profile of network activity (Plumbly et al., 2019). The effects of Diltiazem and Nifedipin were similar to that observed when $\mathrm{GABA}_{\mathrm{A}}$ receptors were inhibited (Plumbly et al., 2019).

\subsubsection{Neurochemical changes}

In addition to other functions, the influx of calcium into the cell regulates neuronal gene expression and neurotransmitter release (West, Griffith and Greenberg, 2002; Devor et al., 2017). Moreover, another hypothesis regarding the pathophysiology of PDs such as SCZ relates to imbalances in the levels of various neurotransmitters (Patel et al., 2014; Sigitova et al., 2017; Langova et al., 2020). A reduction in the mRNA levels of parvalbumin positive cells (a type of inhibitory interneurons) and glutamate decarboxylase 67 (GAD67) (GABA synthesizing enzyme) were reported in the 
dorsolateral prefrontal cortex of postmortem samples of SCZ patients (Hashimoto et al., 2003; Lewis, Hashimoto and Volk, 2005; de Jonge et al., 2017). In this study, HPLC analysis revealed imbalances in the levels of major neurotransmitters in the two cacnalc (sa10930 and sa15296) mutant lines when compared to WT. We found enhanced dopamine and serotonin, decreased GABA and unaffected levels of glutamate in homozygous sa10930. In the homozygous sa15296 larvae, enhanced serotonin and glutamate, unaffected levels of GABA and dopamine were observed. Interestingly, neurotransmitters contribute to neural oscillations and the overall E/I balance needed for proper brain function (Uhlhaas et al., 2008; Uhlhaas and Singer, 2010, 2013). The E/I ratio measured as GLUT/GABA was found to be increased in both sa10930 and sal5296 mutant lines, thus suggesting overall excitability. Consequently, corroborating the results obtained from calculating the slope of the high frequency oscillations that revealed overall neuronal excitability.

LTCCs are shown to facilitate neurotransmitter release through the following: 1) their expression in presynaptic terminals of hippocampal neurons (Tippens et al., 2008; Leitch et al., 2009) and interneurons (Westenbroek, Hoskins and Catterall, 1998), 2) their interaction with the exocytotic machinery either directly or indirectly through the activation of the MAPK ERK1/2 pathway (Wiser, Bennett and Atlas, 1996; Wiser et al., 1999). For example, activation of Cav1.2 in the NAc and Cav1.3 in the VTA activates the CamKII/ERK pathway, which in turn leads to increased phosphorylation of GluA1 (subunit of excitatory AMPA) and subsequent enhancement in GluA1's surface expression (Schierberl et al., 2011). Surprisingly, blockage of LTCCs with nifedipine was reported to result in increased expression of GluR1 via CREB dependent transcription (Gong et al., 2007). Again, multiple evidence support the role of LTCC in dopaminergic transmission by enabling proper dopaminergic function at the terminals (Terrillion et al., 2017) and increasing dopamine burst firing in the VTA (Liu et al., 2014). In their study, Terrillion and colleagues showed that constitutive and conditional Cav1.2 haploinsufficient mice and control mice treated with the LTCC antagonist nimodipine were less responsive to GBR12909 (a DAT blocker), and displayed decreased locomotor response to psychostimulants that elevate dopamine levels (Terrillion et al., 2017). Additionally, activation of Cav1.3 and to a lesser extent Cav1.2 increases dopamine release through the increase of dopamine burst firing in the VTA (Liu et al., 2014). 
Indeed, dysregulation of the mesolimbic-dopamine system produces endophenotypes of SCZ, BP mania and depression (Bhat et al., 2012; Kabir, Martínez-Rivera and Rajadhyaksha, 2017; Sigitova et al., 2017). Psychotropic drugs that either increase the release or decrease the reuptake of dopamine leads to psychotic and manic symptoms (Ecker et al., 2009). On the other hand, antipsychotics and other dopamine antagonists decrease psychotic or manic episodes (Patel et al., 2014; Langova et al., 2020). Similarly, dysregulation in excitatory neurotransmission is associated with psychiatric endophenotypes of SCZ, BP, and depression (de Bartolomeis et al., 2013; Patel et al., 2014; Sigitova et al., 2017). NMDA-R antagonists such as MK-801, phenycycline and ketamine have been shown to induce psychotic like symptoms in animal models and together with dopamine agonists (e.g. apomorphine, amphetamine) are commonly used as pharmacological models of SCZ (de Bartolomeis et al., 2013; Langova et al., 2020).

\subsection{Molecular pathway analysis of cacna1c mutants}

Calcium influx through Cav1.2 calcium channels regulates downstream genetic transcription pathways such as BNDF and C-FOS (Ghosh, Carnahan and Greenberg, 1994; West, Griffith and Greenberg, 2002). For instance, Cav1.2 calcium channels have been shown to preferentially regulate $B D N F$ expression (Ghosh, Carnahan and Greenberg, 1994; West, Griffith and Greenberg, 2002). Polymorphisms in the FOS gene (Boyajyan et al., 2015) and BDNF (Notaras, Hill and van den Buuse, 2015) have been associated with SCZ. As we expected, altered cacnalc function affected the levels of the downstream molecular targets $b d n f$ and $c$-fos in $6 \mathrm{dpf}$ zebrafish larvae.

To begin with, in both sa10930 and sa15296 mutants, there was a statistically significant reduction in the mRNA levels of $b d n f$ i.e. 30 and $50 \%$ decrease respectively when compared to WT siblings. Albeit discordant results sometimes, there are lower levels of BDNF in both medicated and nonmedicated SCZ patients when compared to controls (Favalli et al., 2012). Previous studies have found deficits in the levels of $B d n f$ in mice harbouring forebrain-specific conditional knockout of Cacnalc (Lee et al., 2016), the prefrontal cortex of both Cav1.2 haploinsufficient rats (Cacnalc $+/-)$ and persons with $C A C N A 1 C$ risk-associated genetic variants upon analysis of the BRAINEAC data (Sykes et al., 2019; Tigaret et al., 2021). Indeed, BDNF is associated with synaptic transmission and neural plasticity (Carvalho et al., 2008; 
Gottmann, Mittmann and Lessmann, 2009; Favalli et al., 2012). Concerning synaptic transmission, exposure to or lack of BDNF results in changes to both excitatory and inhibitory synapses (Carvalho et al., 2008; Gottmann, Mittmann and Lessmann, 2009). Bdnf promoter IV-deficient mice $(B d n f-K I V)$ presented significant deficits in parvalbumin expressing GABAergic interneurons in the prefrontal cortex (PFC) (parvalbumin interneurons are associated with executive function and schizophrenia) which resulted in impaired inhibitory synaptic transmission (Sakata et al., 2009). Bdnf-deficient (KO) mice showed enhanced ASR and reduced PPI of ASR (Lima-Ojeda et al., 2019). Haploinsufficient $B d n f$ rats (50 \% Bdnf protein) presented normal PPI at baseline and PPI attenuation was not observed when the rats were challenged with NMDA-R, serotonin-1A receptor agonist, serotonin-2A receptor agonist, serotonin releaser, and dopamine D1/D2 receptor agonist (van den Buuse, Biel and Radscheit, 2017). Heterozygous Bdnf mice (up to $60 \%$ less Bdnf protein) presented normal behavioural phenotype in the locomotor activity, exploration, anxiety, fear-associated learning, and behavioural despair tests, comparable levels of serotonin, norepinephrine, and dopamine but reduced choline acetyltransferase activity (Chourbaji et al., 2004). Heterozygous $B d n f$ mice displayed reduced baseline PPI paradigm (Klug and van den Buuse, 2013; Manning and van den Buuse, 2013; Harb et al., 2021).

In contrast, the level of $c-f o s$ was significantly reduced only in the sa15296 but not the sa10930 mutants - although there was a tendency towards a reduction in the latter mutants. The immediate early gene C-FOS is associated with cell proliferation, survival and death (Boyajyan et al., 2015; Gallo et al., 2018). C-FOS is a marker of neuronal activity, and its dysregulation have been associated with SCZ, learning and memory which is a key signature of many psychiatric disorders and especially in epilepsy (genetic and drug induced seizure animal models) (Zhang et al., 2002; Boyajyan et al., 2015; Gallo et al., 2018; Gawel, Kukula-Koch, et al., 2020). Post-mortem analysis of the brains of patients with schizophrenia revealed that the expression of C-FOS and FOS B was increased in the thalamus (Kyosseva, 2004), whereas C-JUN protein and C-JUN mRNA was significantly increased in the cerebellar vermis (Todorova, Elbein and Kyosseva, 2003). Similarly, elevated levels of $C$-fos has been reported in PTZ-, kainic acid-, and other chemically-induced seizures in animal models (Zhang et al., 2002; Malhi et al., 2014; Gallo et al., 2018; Gawel, Kukula-Koch, et al., 2020). Although C-Fos is widely used as a marker of neuroexcitability in the context of epilepsy, elevation in the levels of $B d n f$ is also considered as an equally valid biomarker (Zhang et al., 2002; Malhi et al., 2014; Gawel, Kukula-Koch, et al., 2020). In our case however, 
whereas the levels of $c$-fos is elevated, the $b d n f$ appears to be downregulated. Thus, we are tempted to further rule out seizures as the cause of the observed increased excitability. Furthermore, MK801 (NMDA-R antagonist used as a pharmacologic model of psychosis) induced expression of $C$ fos mRNA in most brain areas involved in the pathophysiology of SCZ (Väisänen et al., 2004). Conversely, antipsychotic drugs (haloperidol and clozapine) increased the expression of immediate early gene protein c-Fos (Kontkanen, 2002).

\subsection{Zebrafish as a model organism of psychiatric disorders}

The zebrafish has emerged as a useful model to study PDs for several reasons. For instance, zebrafish share about 70\% gene homology with humans (Howe et al., 2013). Using the diverse range of genetic tools, researchers can manipulate the genome and identify how a given gene contributes to disease (Kalueff et al., 2013). Overall, homologous brain structures exist between the zebrafish and the human brain (Panula et al., 2010). Also, numerous behavioural tests relevant to psychiatric disorders have been adapted to zebrafish (Burgess and Granato, 2007b, 2007a; Miller and Gerlai, 2007; Best et al., 2008; Schnörr et al., 2012; Magno et al., 2015). The presence of these phenotypic tools makes zebrafish suitable for phenotypic drug screening which has reemerged as an attractive alternative and complementary approach of drug discovery rather than the traditional target-based screening (Kokel and Peterson, 2008; Rihel et al., 2010; Bruni et al., 2016)

Although non-human animal models provide an opportunity to facilitate our understanding of the functional roles of the various psychiatric risk genes. Admittedly, fish are not humans and can never phenocopy the full spectrum of human disease. However, by focusing on phenotypic aspects of the disease i.e., endophenotypes, it is possible to gain valuable insights into the molecular and cellular functions of these genes.

\subsection{Limitations}

A limitation with the current study is that, extracts from whole larvae were used, which does not allow for the evaluation of tissue- or brain-region specific expression. In addition, we did not assay 
for changes over time - i.e., across developmental stages. While we identified a significant reduction in mutant mRNA, integrating protein level data such western blot analysis would provide better evidence as to the effect of the mutation on gene expression i.e., LOF or GOF (Buccitelli and Selbach, 2020). Also, it would be favourable to incorporate experiments such as whole-mount in situ hybridisation analysis that would allow for both temporal and spatial expression analysis, given that LTCCs expression is developmentally regulated and differs across tissues (Bhat et al., 2012; Lipscombe and Andrade, 2015).

GWAS highlight candidate loci and genes thought to contribute to disease and quantitative traits. However, they do not specify the functional variant on each locus, although this limitation can be partially overcome by analysing gene enrichment pathways (Dourlen, Chapuis and Lambert, 2018). To identify the causal genes and understand their contribution to disease or trait pathogenesis, in vitro and in vivo strategies have been deployed. Over the years, animal models with LOF or GOF of single genes have been useful in providing information about the functions of some of these identified genes, but this approach may not be robust enough considering the high number of GWAS implicated genes. One of the suggestions on how to overcome this inherent GWAS challenge is the use of a systems genetics approach as shown recently in the field of diabetes (Herman, Campbell and D'Alessio, 2019; Keller et al., 2019). By taking advantage of the numerous genetic tools available, it is possible to generate transgenic lines carrying patientspecific mutations in $C A C N A 1 C$ or $C A C N A 1 D$ or edit multiple gene loci. This is possible with Crispr/Cas9 mutagenesis, or transgenesis, where patient variants are expressed in zebrafish (our own unpublished data). Rescue of zebrafish mutant phenotypes through expression of the WT human orthologue can also be achieved [(Schubert et al., 2014) and our unpublished data on other zebrafish mutants].

Furthermore, functional connectivity deficits are associated with several PDs including SCZ (Dawson, Morris and Pratt, 2015). In our quest to investigate some of the functional deficits in our mutants, single electrode LFP recordings were performed. The use of single electrodes limits the spatial resolution of the neural signals one can detect. In our experimental setup, we focused on recording from the optic tectum, which carries out functions roughly equivalent to the mammalian neocortex and visual cortical functions. Notably, the zebrafish forebrain contains important mammalian cortical homologous structures (isocortex), which underlie most psychiatric 
phenotypes. However, accessing and recording from this region of the larval zebrafish brain is more difficult and the methodology has only recently been established in our laboratory. We therefore chose to record from the optic tectum for the purpose of this study. In the absence of multi-electrodes which can facilitate simultaneous recordings from multiple brain regions, an alternative would be to probe other interesting brain areas (e.g., cerebellum or forebrain) to assess the functional phenotypes. Using neuronal activity markers such as pErk and c-fos could also provide valuable insights into functional connectivity phenotypes in zebrafish.

\subsection{Future perspectives}

Morphological brain changes have been reported in persons with PDs such as SCZ, BP and ASD (Krystal and State, 2014). Similarly, morphological changes in the brain, have also been found in individuals carrying $C A C N A 1 C$ psychiatric risk variants (Kempton et al., 2009; Bigos et al., 2010; Franke et al., 2010; Tesli et al., 2013). In the future, it will be important to investigate potential changes in neuroplasticity as a result of altered LTCC function, by examining alterations in neuronal architecture (e.g., dendritic arborisation) or circuitry (e.g., tracing of neuronal projections, calcium activity) in the brain using cell-specific fluorescent reporters, calcium imaging, light-sheet microscopy or confocal microscopy. Although the findings of the thesis suggest alteration in the E/I balance of the mutant larvae studied based on LFP and neurochemical data, one can further examine changes at the cellular level by monitoring the survival, proliferation or migration of specific cell populations such as dopaminergic, glutamatergic, or GABAergic neurons, as well as glial cell populations. Indeed, several zebrafish transgenic lines are available for such studies.

We have identified several phenotypes across behavioural, gene expression, neural oscillations and neurochemical domains in our mutants. Most of the behavioural phenotypes are known to be modulated by psychoactive drugs. Future studies may focus on investigating whether some of the known drugs would improve/reverse the behavioural deficits observed in our mutants. Furthermore, the downstream pathways $b d n f$ and $c$-fos that were observed to be differentially expressed could be targeted pharmacologically to test their role in the gross phenotypic defects observed in our mutants. Recently, Tigaret et al., reported behavioural and synaptic plasticity 
deficits as well as reduced immunoreactivity of phosphorylated Erk and CREB in Cacnalc+/rats. By activating the aforementioned pathways with a small-molecule agonist of TrkB/TrkC neurotrophin receptors, they were able to rescue the observed behavioural and synaptic plasticity deficits in their Cacnalc haploinsufficient rats (Tigaret et al., 2021).

There are currently a number of competing hypotheses of SCZ pathophysiology (similar to other PDs) that seem mutually exclusive - neurotransmitter dysregulation (involving dopamine, glutamate, or GABA), calcium homeostasis or second messenger signalling disruption, growth factor deficiency (Devor et al., 2017). The existing complexity has not been resolved. Rather, GWAS findings continues to identify genes involved in all of the aforementioned processes (Devor et al., 2017). RNASeq and other "omics" data analysis are useful for providing global information on gene regulatory changes caused by disease or treatment, which is advantageous over selective marker analysis using qPCR, the latter of which does not provide the true scale of events, instead only providing a snapshot of changes that may be attributable to the genetic insult, disease or treatment (Nguyen et al., 2019). The current consensus is that simply measuring gene expression changes is not sufficient to predict or explain mechanisms underlying the observed biological phenomena (The Network and Pathway Analysis Subgroup of the Psychiatric Genomic Consortium, 2015; Nguyen et al., 2019). Hence, pathway enrichment analysis has been suggested as a useful method that provides mechanistic insight into biological pathways enriched in different "omics" data set relative to what is expected by chance (Nguyen et al., 2019; Reimand et al., 2019). To gain insights into pathways regulated by the global deletion or haploinsufficiency of our cacnalc and/or cacnalda mutants, RNASeq could therefore be pursued. Pathway analysis of ASD, SCZ and GWAS datasets have pointed to various biological pathways that may be involved in disease pathogenesis (De Rubeis et al., 2014; Fromer et al., 2014; Purcell et al., 2014; Pocklington et al., 2015; The Network and Pathway Analysis Subgroup of the Psychiatric Genomic Consortium, 2015).

The small size of zebrafish larvae make them highly amenable to in vivo drug screens in a multiwell format (Rihel and Schier, 2012). High-throughput drug screens have been successfully carried out in larval zebrafish taking advantage of the wide repertoire of behavioural tests and automated systems available. Kokel and colleagues screened a large drug library using the photomotor response (PMR) test, a simple locomotor assay where response to light flash is measured in the 
larval zebrafish. In this study, the authors observed distinct and reproducible effects of many psychoactive compounds while similar PMR phenotypes were reported for drugs that have similar targets in humans (Kokel et al., 2010). Following reports that had shown that larval zebrafish locomotion is altered by sleep-wake neuropeptides (Prober et al., 2006; Woods et al., 2014), Rihel et al., sought to find new drugs that influence sleep-wake behaviour in larval zebrafish. The study was conducted in a high-throughput basis and it revealed that drugs with shared phenotypes also had similar human indications and biochemical mechanisms (Rihel et al., 2010). Moving forward, it would be useful to capitalise on the models described herein for use in carrying out a chemical modifier screen using either novel compounds or to repurpose existing FDA/EMA approved drugs. The primary screen could utilise the validated PPI protocol as a starting point. Identified hits from the primary screen can be pursued further using functional imaging or neurochemical techniques with the hope that the results would inspire development of novel therapeutic approaches with the potential to improve the quality of life of persons suffering from PDs. 


\subsection{CONCLUSION}

The goals of this thesis were three-fold: 1) to establish and validate a robust PPI assay platform for use in characterising zebrafish mutant lines, 2) to examine the effects of cacnalc mutations on zebrafish early brain function and 3) to examine the effect of cacnalda mutation on zebrafish early brain function.

In lieu of aim 1, our results showed that by combining ZebraBox Revo + EthoVision, it was possible to accurately design a testing paradigm for performing the PPI test in zebrafish larvae.

The remaining two aims sought to examine the roles of cacnalc and cacnalda in larval zebrafish brain function. The two neuronal LTCC subtypes are involved in several processes relevant to PDs such as learning, memory, and brain development (Kabir, Martínez-Rivera and Rajadhyaksha, 2017; Moon et al., 2018). Indeed, they are thought to contribute to the proper functioning of the brain circuitry i.e. the mesolimbic system, hippocampus, and amygdala, which are implicated in the pathophysiology of various PDs (Bhat et al., 2012; Zamponi, 2016). Hence, our hypothesis that aberration in calcium signalling in the context of CACNAIC and CACNA1D can be detrimental to brain function.

To our knowledge, paper III is the first time that a study has characterised the morphologic, cacnalda mRNA expression and behavioural effects of a splice variant mutation in the zebrafish cacnalda. Additionally, paper IV makes a significant contribution in our understanding of how different aberrations in the cacnalc can lead to changes in phenotypes associated with normal brain function, thus, supporting the argument for the in vivo analysis of psychiatric risk genes across broad phenotypic domains, and not based on stringent disorder based classification systems. Together, we show that mutations that alter the function of cacnalda and cacnalc in zebrafish lead to a wide-range of phenotypes in larvae. The reported phenotypes are reminiscent of relevant functional domains observed in PDs. Specifically, genetically-induced deficiency in cacnalc may contribute to endophenotypes related to PDs by altering downstream molecular targets - in this case, $b d n f$ and $c$-fos. 


\section{REFERENCES}

Abramowicz, A. and Gos, M. (2018) 'Splicing mutations in human genetic disorders: examples, detection, and confirmation', Journal of Applied Genetics, 59(3), pp. 253-268. doi:

10.1007/s13353-018-0444-7.

Afrikanova, T. et al. (2013) 'Validation of the zebrafish pentylenetetrazol seizure model: locomotor versus electrographic responses to antiepileptic drugs', PloS One, 8(1), p. e54166. doi: 10.1371/journal.pone.0054166.

Aleström, P. et al. (2019) 'Zebrafish: Housing and husbandry recommendations', Laboratory Animals. doi: 10.1177/0023677219869037.

Ament, S. A. et al. (2015) 'Rare variants in neuronal excitability genes influence risk for bipolar disorder', Proceedings of the National Academy of Sciences, 112(11), pp. 3576-3581. doi: 10.1073/pnas.1424958112.

Andrade, A. et al. (2019) 'Genetic Associations between Voltage-Gated Calcium Channels and Psychiatric Disorders', International Journal of Molecular Sciences, 20(14). doi: 10.3390/ijms20143537.

Aranda, P. S., LaJoie, D. M. and Jorcyk, C. L. (2012) 'Bleach gel: A simple agarose gel for analyzing RNA quality', ELECTROPHORESIS, 33(2), pp. 366-369. doi:

10.1002/elps.201100335.

Bader, P. L. et al. (2011) 'Mouse model of Timothy syndrome recapitulates triad of autistic traits', Proceedings of the National Academy of Sciences, 108(37), pp. 15432-15437. doi: 10.1073/pnas.1112667108.

Baig, S. M. et al. (2011) 'Loss of Cav1.3 (CACNA1D) function in a human channelopathy with bradycardia and congenital deafness', Nature Neuroscience, 14(1), pp. 77-84. doi:

10.1038/nn.2694.

Banono, N. S. et al. (2021) 'Zebrafish Larvae Carrying a Splice Variant Mutation in cacnald: A New Model for Schizophrenia-Like Behaviours?', Molecular Neurobiology, 58(2), pp. 877-894. doi: $10.1007 / \mathrm{s} 12035-020-02160-5$.

Banono, N. S. and Esguerra, C. V. (2020) 'Pharmacological Validation of the Prepulse Inhibition of Startle Response in Larval Zebrafish using a Commercial Automated System and Software', Journal of Visualized Experiments, (161), p. 61423. doi: 10.3791/61423.

Baraban, S. C. et al. (2005) 'Pentylenetetrazole induced changes in zebrafish behavior, neural activity and c-fos expression', Neuroscience, 131(3), pp. 759-768. doi:

10.1016/j.neuroscience.2004.11.031. 
Baraban, S. C., Dinday, M. T. and Hortopan, G. A. (2013) 'Drug screening in Scn1a zebrafish mutant identifies clemizole as a potential Dravet syndrome treatment', Nature Communications, 4, p. 2410. doi: 10.1038/ncomms3410.

Barbado, M. et al. (2009) 'Gene regulation by voltage-dependent calcium channels', Biochimica et Biophysica Acta (BBA) - Molecular Cell Research, 1793(6), pp. 1096-1104. doi: 10.1016/j.bbamcr.2009.02.004.

Barros, V. N. et al. (2015) 'The pattern of c-Fos expression and its refractory period in the brain of rats and monkeys', Frontiers in Cellular Neuroscience, 9. doi: 10.3389/fncel.2015.00072.

de Bartolomeis, A. et al. (2013) 'Different effects of the NMDA receptor antagonists ketamine, MK-801, and memantine on postsynaptic density transcripts and their topography: role of Homer signaling, and implications for novel antipsychotic and pro-cognitive targets in psychosis', Progress in Neuro-Psychopharmacology \& Biological Psychiatry, 46, pp. 1-12. doi: 10.1016/j.pnpbp.2013.06.010.

Basnet, R. M. et al. (2019) 'Zebrafish Larvae as a Behavioral Model in Neuropharmacology', Biomedicines, 7(1), p. 23. doi: 10.3390/biomedicines7010023.

Best, J. D. et al. (2008) 'Non-Associative Learning in Larval Zebrafish', Neuropsychopharmacology, 33(5), pp. 1206-1215. doi: 10.1038/sj.npp.1301489.

Bhandiwad, A. A. et al. (2013) 'Auditory sensitivity of larval zebrafish (Danio rerio) measured using a behavioral prepulse inhibition assay', The Journal of Experimental Biology, 216(Pt 18), pp. 3504-3513. doi: 10.1242/jeb.087635.

Bhat, S. et al. (2012) 'CACNA1C (Cav1.2) in the pathophysiology of psychiatric disease', Progress in neurobiology, 99(1), pp. 1-14. doi: 10.1016/j.pneurobio.2012.06.001.

Biała, G. (2003) 'Calcium channel antagonists suppress nicotine-induced place preference and locomotor sensitization in rodents', Polish Journal of Pharmacology, 55(3), pp. 327-335.

Biała, G. and Kruk, M. (2007) 'Amphetamine-induced anxiety-related behavior in animal models', Pharmacological reports: PR, 59(6), pp. 636-644.

Bigos, K. L. et al. (2010) 'Genetic Variation in CACNA1C Affects Brain Circuitries Related to Mental Illness', Archives of General Psychiatry, 67(9), p. 939. doi:

10.1001/archgenpsychiatry.2010.96.

Boczek, N. J. et al. (2015) 'Novel Timothy syndrome mutation leading to increase in CACNA1C window current', Heart Rhythm, 12(1), pp. 211-219. doi: 10.1016/j.hrthm.2014.09.051.

Boyajyan, A. et al. (2015) 'Schizophrenia-associated Risk and Protective Variants of c-Fos Encoding Gene', Recent advances in DNA \& gene sequences, 9(1), pp. 51-57. doi: $10.2174 / 2352092209666150223113334$. 
Bozarth, X. et al. (2018) 'Expanding clinical phenotype in CACNA1C related disorders: From neonatal onset severe epileptic encephalopathy to late-onset epilepsy', American Journal of Medical Genetics Part A, 176(12), pp. 2733-2739. doi: 10.1002/ajmg.a.40657.

Bruni, G. et al. (2016) 'Zebrafish behavioral profiling identifies multitarget antipsychotic-like compounds', Nature Chemical Biology, 12(7), pp. 559-566. doi: 10.1038/nchembio.2097.

Buccitelli, C. and Selbach, M. (2020) 'mRNAs, proteins and the emerging principles of gene expression control', Nature Reviews Genetics, 21(10), pp. 630-644. doi: 10.1038/s41576-0200258-4.

Burgess, H. A. and Granato, M. (2007a) 'Modulation of locomotor activity in larval zebrafish during light adaptation', The Journal of Experimental Biology, 210(Pt 14), pp. 2526-2539. doi: $10.1242 /$ jeb.003939.

Burgess, H. A. and Granato, M. (2007b) 'Sensorimotor gating in larval zebrafish', The Journal of Neuroscience: The Official Journal of the Society for Neuroscience, 27(18), pp. 4984-4994. doi: 10.1523/JNEUROSCI.0615-07.2007.

Burmeister, M., McInnis, M. G. and Zöllner, S. (2008) 'Psychiatric genetics: progress amid controversy’, Nature Reviews Genetics, 9(7), pp. 527-540. doi: 10.1038/nrg2381.

Busquet, P. et al. (2010) 'CaV1.3 L-type Ca2+ channels modulate depression-like behaviour in mice independent of deaf phenotype', International Journal of Neuropsychopharmacology, 13(4), pp. 499-513. doi: 10.1017/S1461145709990368.

van den Buuse, M. (2010) 'Modeling the Positive Symptoms of Schizophrenia in Genetically Modified Mice: Pharmacology and Methodology Aspects', Schizophrenia Bulletin, 36(2), pp. 246-270. doi: 10.1093/schbul/sbp132.

van den Buuse, M., Biel, D. and Radscheit, K. (2017) 'Does genetic BDNF deficiency in rats interact with neurotransmitter control of prepulse inhibition? Implications for schizophrenia', Progress in Neuro-Psychopharmacology and Biological Psychiatry, 75, pp. 192-198. doi: 10.1016/j.pnpbp.2017.02.009.

Calcagnetti, D. J. and Schechter, M. D. (1992) 'Attenuation of drinking sweetened water following calcium channel blockade', Brain Research Bulletin, 28(6), pp. 967-973. doi: 10.1016/0361-9230(92)90219-N.

do Carmo Silva, R. X., Lima-Maximino, M. G. and Maximino, C. (2018) 'The aversive brain system of teleosts: Implications for neuroscience and biological psychiatry', Neuroscience \& Biobehavioral Reviews, 95, pp. 123-135. doi: 10.1016/j.neubiorev.2018.10.001.

Carvalho, A. L. et al. (2008) 'Role of the brain-derived neurotrophic factor at glutamatergic synapses', British Journal of Pharmacology, 153(S1), pp. S310-S324. doi:

https://doi.org/10.1038/sj.bjp.0707509. 
Catterall, W. A. (2011) 'Voltage-Gated Calcium Channels', Cold Spring Harbor Perspectives in Biology, 3(8), pp. a003947-a003947. doi: 10.1101/cshperspect.a003947.

Chartoff, E. H., Pliakas, A. M. and Carlezon, W. A. (2006) 'Microinjection of the L-Type Calcium Channel Antagonist Diltiazem into the Ventral Nucleus Accumbens Shell Facilitates Cocaine-Induced Conditioned Place Preferences', Biological Psychiatry, 59(12), pp. 1236-1239. doi: 10.1016/j.biopsych.2005.09.024.

Chourbaji, S. et al. (2004) 'Mice with reduced brain-derived neurotrophic factor expression show decreased choline acetyltransferase activity, but regular brain monoamine levels and unaltered emotional behavior', Molecular Brain Research, 121(1-2), pp. 28-36. doi: 10.1016/j.molbrainres.2003.11.002.

Colwill, R. M. and Creton, R. (2011) 'Imaging escape and avoidance behavior in zebrafish larvae', Reviews in the Neurosciences, 22(1). doi: 10.1515/rns.2011.008.

Cross-Disorder Group of the Psychiatric Genomics Consortium (2013) 'Identification of risk loci with shared effects on five major psychiatric disorders: a genome-wide analysis', The Lancet, 381(9875), pp. 1371-1379. doi: 10.1016/S0140-6736(12)62129-1.

Cuthbert, B. N. and Insel, T. R. (2013) 'Toward the future of psychiatric diagnosis: the seven pillars of RDoC', BMC Medicine, 11(1), p. 126. doi: 10.1186/1741-7015-11-126.

Dao, D. T. et al. (2010) 'Mood Disorder Susceptibility Gene CACNA1C Modifies MoodRelated Behaviors in Mice and Interacts with Sex to Influence Behavior in Mice and Diagnosis in Humans', Biological Psychiatry, 68(9), pp. 801-810. doi: 10.1016/j.biopsych.2010.06.019.

Dawson, N., Morris, B. J. and Pratt, J. A. (2015) 'Functional brain connectivity phenotypes for schizophrenia drug discovery', Journal of Psychopharmacology, 29(2), pp. 169-177. doi: $10.1177 / 0269881114563635$.

De Rubeis, S. et al. (2014) 'Synaptic, transcriptional and chromatin genes disrupted in autism', Nature, 515(7526), pp. 209-215. doi: 10.1038/nature13772.

Devor, A. et al. (2017) 'Genetic evidence for role of integration of fast and slow neurotransmission in schizophrenia', Molecular psychiatry, 22(6), pp. 792-801. doi: 10.1038/mp.2017.33.

Dolphin, A. C. (2018) 'Voltage-gated calcium channels: Their discovery, function and importance as drug targets', Brain and Neuroscience Advances, 2, p. 239821281879480. doi: $10.1177 / 2398212818794805$.

Dourlen, P., Chapuis, J. and Lambert, J.-C. (2018) 'Using High-Throughput Animal or CellBased Models to Functionally Characterize GWAS Signals', Current Genetic Medicine Reports, 6(3), pp. 107-115. doi: 10.1007/s40142-018-0141-1. 
Duan, A. R. et al. (2015) 'Delta Frequency Optogenetic Stimulation of the Thalamic Nucleus Reuniens Is Sufficient to Produce Working Memory Deficits: Relevance to Schizophrenia', Biological Psychiatry, 77(12), pp. 1098-1107. doi: 10.1016/j.biopsych.2015.01.020.

Ecker, D. et al. (2009) 'Dopamine Agonists and their risk to induce psychotic episodes in Parkinson's disease: a case-control study', BMC Neurology, 9, p. 23. doi: 10.1186/1471-2377-923.

Favalli, G. et al. (2012) 'The role of BDNF in the pathophysiology and treatment of schizophrenia', Journal of Psychiatric Research, 46(1), pp. 1-11. doi: 10.1016/j.jpsychires.2011.09.022.

Ferreira, M. A. R. et al. (2008) 'Collaborative genome-wide association analysis supports a role for ANK3 and CACNA1C in bipolar disorder', Nature Genetics, 40(9), pp. 1056-1058. doi: 10.1038/ng.209.

Fish, B. et al. (1992) 'Infants at risk for schizophrenia: sequelae of a genetic neurointegrative defect. A review and replication analysis of pandysmaturation in the Jerusalem Infant Development Study', Archives of General Psychiatry, 49(3), pp. 221-235.

Ford, K. A., Wolf, M. E. and Hu, X.-T. (2009) 'Plasticity of L-type $\mathrm{Ca}^{2+}$ channels after cocaine withdrawal', Synapse, 63(8), pp. 690-697. doi: 10.1002/syn.20651.

Franke, B. et al. (2010) 'Genetic Variation in CACNA1C, a Gene Associated with Bipolar Disorder, Influences Brainstem Rather than Gray Matter Volume in Healthy Individuals', Biological Psychiatry, 68(6), pp. 586-588. doi: 10.1016/j.biopsych.2010.05.037.

Freeze, B. S., McNulty, M. M. and Hanck, D. A. (2006) 'State-dependent verapamil block of the cloned human $\mathrm{Ca}(\mathrm{v}) 3.1 \mathrm{~T}$-type $\mathrm{Ca}(2+)$ channel', Molecular Pharmacology, 70(2), pp. 718-726. doi: $10.1124 / \mathrm{mol} .106 .023473$.

Freir, D. B. and Herron, C. E. (2003) 'Inhibition of 1-type voltage dependent calcium channels causes impairment of long-term potentiation in the hippocampal CA1 region in vivo', Brain Research, 967(1-2), pp. 27-36. doi: 10.1016/S0006-8993(02)04190-2.

Fromer, M. et al. (2014) 'De novo mutations in schizophrenia implicate synaptic networks', Nature, 506(7487), pp. 179-184. doi: 10.1038/nature12929.

Fulga, I. G. and Stroescu, V. (1997) 'Experimental research on the effect of calcium channel blockers nifedipine and verapamil on the anxiety in mice', Romanian Journal of Physiology: Physiological Sciences, 34(1-4), pp. 127-136.

Gallo, F. T. et al. (2018) 'Immediate Early Genes, Memory and Psychiatric Disorders: Focus on c-Fos, Egr1 and Arc', Frontiers in Behavioral Neuroscience, 12, p. 79. doi: 10.3389/fnbeh.2018.00079.

Gao, R., Peterson, E. J. and Voytek, B. (2017) 'Inferring synaptic excitation/inhibition balance from field potentials', NeuroImage, 158, pp. 70-78. doi: 10.1016/j.neuroimage.2017.06.078. 
Gawel, K., Turski, W. A., et al. (2020) 'Phenotypic Characterization of Larval Zebrafish (Danio rerio) with Partial Knockdown of the cacnal a Gene', Molecular Neurobiology, 57(4), pp. 1904 1916. doi: 10.1007/s12035-019-01860-x.

Gawel, K., Kukula-Koch, W., et al. (2020) 'The Influence of Palmatine Isolated from Berberis sibirica Radix on Pentylenetetrazole-Induced Seizures in Zebrafish', Cells, 9(5), p. 1233. doi: 10.3390/cells9051233.

Gaynes, B. N. et al. (2009) 'What Did STAR*D Teach Us? Results From a Large-Scale, Practical, Clinical Trial for Patients With Depression', Psychiatric Services, 60(11), pp. 14391445. doi: 10.1176/ps.2009.60.11.1439.

Geng, Y. and Peterson, R. T. (2019) 'The zebrafish subcortical social brain as a model for studying social behavior disorders', Disease Models \& Mechanisms, 12(8), p. dmm039446. doi: 10.1242/dmm.039446.

Gerlai, R. (2019) 'Reproducibility and replicability in zebrafish behavioral neuroscience research', Pharmacology Biochemistry and Behavior, 178, pp. 30-38. doi: 10.1016/j.pbb.2018.02.005.

Gershon, E. S. et al. (2014) 'A rare mutation of CACNA1C in a patient with bipolar disorder, and decreased gene expression associated with a bipolar-associated common SNP of CACNA1C in brain', Molecular Psychiatry, 19(8), pp. 890-894. doi: 10.1038/mp.2013.107.

Ghosh, A., Carnahan, J. and Greenberg, M. (1994) 'Requirement for BDNF in activitydependent survival of cortical neurons', Science, 263(5153), pp. 1618-1623. doi:

10.1126/science.7907431.

Gillis, J. et al. (2012) 'Long QT, syndactyly, joint contractures, stroke and novel CACNA1C mutation: Expanding the spectrum of Timothy syndrome', American Journal of Medical Genetics Part A, 158A(1), pp. 182-187. doi: 10.1002/ajmg.a.34355.

Gong, B. et al. (2007) 'Genetic evidence for the requirement of adenylyl cyclase 1 in synaptic scaling of forebrain cortical neurons', European Journal of Neuroscience, 26(2), pp. 275-288. doi: $10.1111 / j .1460-9568.2007 .05669 . x$.

Goonasekera, S. A. et al. (2012) 'Decreased cardiac L-type Ca2+ channel activity induces hypertrophy and heart failure in mice', The Journal of Clinical Investigation, 122(1), pp. 280 290. doi: 10.1172/JCI58227.

Gottmann, K., Mittmann, T. and Lessmann, V. (2009) 'BDNF signaling in the formation, maturation and plasticity of glutamatergic and GABAergic synapses', Experimental Brain Research, 199(3), pp. 203-234. doi: 10.1007/s00221-009-1994-z.

Gould, T. D. and Gottesman, I. I. (2006) 'Psychiatric endophenotypes and the development of valid animal models', Genes, Brain and Behavior, 5(2), pp. 113-119. doi: 10.1111/j.1601183X.2005.00186.x. 
Granato, M. et al. (1996) 'Genes controlling and mediating locomotion behavior of the zebrafish embryo and larva’, Development, 123(1), pp. 399-413.

Grande, I. et al. (2016) 'Bipolar disorder', Lancet (London, England), 387(10027), pp. 15611572. doi: 10.1016/S0140-6736(15)00241-X.

Guan, F. et al. (2015) 'Evaluation of genetic susceptibility of common variants in CACNA1D with schizophrenia in Han Chinese', Scientific Reports, 5, p. 12935. doi: 10.1038/srep12935.

Haesemeyer, M. and Schier, A. F. (2015) 'The study of psychiatric disease genes and drugs in zebrafish', Current opinion in neurobiology, 0, pp. 122-130. doi: 10.1016/j.conb.2014.12.002.

Harb, M. et al. (2021) 'BDNF haploinsufficiency induces behavioral endophenotypes of schizophrenia in male mice that are rescued by enriched environment', Translational Psychiatry, 11(1), pp. 1-13. doi: 10.1038/s41398-021-01365-z.

Harrison, P. J. (2015) 'Recent genetic findings in schizophrenia and their therapeutic relevance', Journal of Psychopharmacology, 29(2), pp. 85-96. doi: 10.1177/0269881114553647.

Hashimoto, T. et al. (2003) 'Gene expression deficits in a subclass of GABA neurons in the prefrontal cortex of subjects with schizophrenia', The Journal of Neuroscience: The Official Journal of the Society for Neuroscience, 23(15), pp. 6315-6326.

Haverinen, J. et al. (2018) 'Expression of calcium channel transcripts in the zebrafish heart: dominance of T-type channels', Journal of Experimental Biology, 221(10), p. jeb179226. doi: 10.1242/jeb.179226.

Herman, M. A., Campbell, J. E. and D'Alessio, D. A. (2019) 'One small step for mice, one giant leap for GWAS?', Journal of Clinical Investigation, 129(10), pp. 4083-4085. doi:

10.1172/JCI131650.

Hetzenauer, A. et al. (2006) 'Brain activation pattern induced by stimulation of L-type Ca2+channels: Contribution of CaV1.3 and CaV1.2 isoforms', Neuroscience, 139(3), pp. 1005-1015. doi: 10.1016/j.neuroscience.2006.01.059.

Heyes, S. et al. (2015) 'Genetic disruption of voltage-gated calcium channels in psychiatric and neurological disorders', Progress in Neurobiology, 134, pp. 36-54. doi:

10.1016/j.pneurobio.2015.09.002.

Hill, A. J. et al. (2004) 'Water permeability and TCDD-induced edema in zebrafish early-life stages', Toxicological Sciences: An Official Journal of the Society of Toxicology, 78(1), pp. 7887. doi: $10.1093 /$ toxsci/kfh056.

Hofer, N. T. et al. (2020) 'Biophysical classification of a CACNA1D de novo mutation as a high-risk mutation for a severe neurodevelopmental disorder', Molecular Autism, 11. doi: 10.1186/s13229-019-0310-4. 
Hotz, A. L. et al. (2021) Loss of the glial glutamate transporter eaat2a leads to a combined developmental and epileptic encephalopathy in zebrafish. preprint. Neuroscience. doi: 10.1101/2021.03.16.435577.

Howe, K. et al. (2013) 'The zebrafish reference genome sequence and its relationship to the human genome', Nature, 496(7446), pp. 498-503. doi: 10.1038/nature12111.

Insel, T. R. (2009) 'Disruptive insights in psychiatry: transforming a clinical discipline', Journal of Clinical Investigation, 119(4), pp. 700-705. doi: 10.1172/JCI38832.

Insel, T. R. (2010a) 'Rethinking Mental Illness', JAMA, 303(19), p. 1970. doi: 10.1001/jama.2010.555.

Insel, T. R. (2010b) 'Rethinking schizophrenia', Nature, 468(7321), pp. 187-193. doi: 10.1038 /nature09552.

Insel, T. R. (2012) 'Next-Generation Treatments for Mental Disorders', Science Translational Medicine, 4(155), pp. 155ps19-155ps19. doi: 10.1126/scitranslmed.3004873.

Iossifov, I. et al. (2012) 'De Novo Gene Disruptions in Children on the Autistic Spectrum', Neuron, 74(2), pp. 285-299. doi: 10.1016/j.neuron.2012.04.009.

Irons, T. D. et al. (2013) 'Acute Administration of Dopaminergic Drugs has Differential Effects on Locomotion in Larval Zebrafish', Pharmacology, biochemistry, and behavior, 103(4), pp. 792-813. doi: 10.1016/j.pbb.2012.12.010.

Jeon, D. et al. (2010) 'Observational fear learning involves affective pain system and Cav1.2 Ca2+ channels in ACC', Nature Neuroscience, 13(4), pp. 482-488. doi: 10.1038/nn.2504.

de Jonge, J. C. et al. (2017) 'GABAergic Mechanisms in Schizophrenia: Linking Postmortem and In Vivo Studies', Frontiers in Psychiatry, 8. doi: 10.3389/fpsyt.2017.00118.

Kabir, Z. D. et al. (2017) 'Rescue of impaired sociability and anxiety-like behavior in adult cacnalc-deficient mice by pharmacologically targeting eIF2 $\alpha$ ', Molecular Psychiatry, 22(8), pp. 1096-1109. doi: 10.1038/mp.2017.124.

Kabir, Z. D., Martínez-Rivera, A. and Rajadhyaksha, A. M. (2017) 'From Gene to Behavior: LType Calcium Channel Mechanisms Underlying Neuropsychiatric Symptoms', Neurotherapeutics: The Journal of the American Society for Experimental NeuroTherapeutics, 14(3), pp. 588-613. doi: 10.1007/s13311-017-0532-0.

Kabitzke, P. A. et al. (2018) 'Comprehensive analysis of two Shank3 and the Cacnalc mouse models of autism spectrum disorder: Shank3 and Cacna1c Models of ASD', Genes, Brain and Behavior, 17(1), pp. 4-22. doi: 10.1111/gbb.12405.

Kalueff, A. V. et al. (2013) 'Towards a Comprehensive Catalog of Zebrafish Behavior 1.0 and Beyond', Zebrafish, 10(1), pp. 70-86. doi: 10.1089/zeb.2012.0861. 
Kalueff, A. V., Stewart, A. M. and Gerlai, R. (2014) 'Zebrafish as an emerging model for studying complex brain disorders', Trends in Pharmacological Sciences, 35(2), pp. 63-75. doi: 10.1016/j.tips.2013.12.002.

Kam, J. W. Y. et al. (2013) 'Resting state EEG power and coherence abnormalities in bipolar disorder and schizophrenia', Journal of Psychiatric Research, 47(12), pp. 1893-1901. doi: 10.1016/j.jpsychires.2013.09.009.

Kedra, M. et al. (2020) 'TrkB hyperactivity contributes to brain dysconnectivity, epileptogenesis, and anxiety in zebrafish model of Tuberous Sclerosis Complex', Proceedings of the National Academy of Sciences, 117(4), pp. 2170-2179. doi: 10.1073/pnas.1910834117.

Keller, M. P. et al. (2019) 'Gene loci associated with insulin secretion in islets from nondiabetic mice', Journal of Clinical Investigation, 129(10), pp. 4419-4432. doi: 10.1172/JCI129143.

Kelly, T. M. and Daley, D. C. (2013) 'Integrated Treatment of Substance Use and Psychiatric Disorders', Social Work in Public Health, 28(3-4), pp. 388-406. doi: 10.1080/19371918.2013.774673.

Kempton, M. J. et al. (2009) 'Effects of the CACNA1C Risk Allele for Bipolar Disorder on Cerebral Gray Matter Volume in Healthy Individuals', American Journal of Psychiatry, 166(12), pp. 1413-1414. doi: 10.1176/appi.ajp.2009.09050680.

Kettleborough, R. N. W. et al. (2013) 'A systematic genome-wide analysis of zebrafish proteincoding gene function’, Nature, 496(7446), pp. 494-497. doi: 10.1038/nature11992.

Klassen, T. et al. (2011) 'Exome Sequencing of Ion Channel Genes Reveals Complex Profiles Confounding Personal Risk Assessment in Epilepsy', Cell, 145(7), pp. 1036-1048. doi: 10.1016/j.cell.2011.05.025.

Klug, M. and van den Buuse, M. (2013) 'An investigation into "two hit" effects of BDNF deficiency and young-adult cannabinoid receptor stimulation on prepulse inhibition regulation and memory in mice', Frontiers in Behavioral Neuroscience, 7, p. 149. doi:

10.3389/fnbeh.2013.00149.

Kokel, D. et al. (2010) 'Rapid behavior-based identification of neuroactive small molecules in the zebrafish', Nature Chemical Biology, 6(3), pp. 231-237. doi: 10.1038/nchembio.307.

Kokel, D. and Peterson, R. T. (2008) 'Chemobehavioural phenomics and behaviour-based psychiatric drug discovery in the zebrafish', Briefings in Functional Genomics \& Proteomics, 7(6), pp. 483-490. doi: 10.1093/bfgp/eln040.

Konopaske, G. T. et al. (2014) 'Prefrontal Cortical Dendritic Spine Pathology in Schizophrenia and Bipolar Disorder', JAMA Psychiatry, 71(12), p. 1323. doi:

10.1001/jamapsychiatry.2014.1582. 
Kontkanen, O. (2002) 'Chronic Antipsychotic Drug Treatment Induces Long-lasting Expression of fos and jun Family Genes and Activator Protein 1 Complex in the Rat Prefrontal Cortex', Neuropsychopharmacology, 27(2), pp. 152-162. doi: 10.1016/S0893-133X(02)00289-0.

Koschak, A. et al. (2001) ' $\alpha 1 \mathrm{D}$ (Cav1.3) Subunits Can Form L-type Ca2+ Channels Activating at Negative Voltages*', Journal of Biological Chemistry, 276(25), pp. 22100-22106. doi: 10.1074/jbc.M101469200.

Krystal, J. H. and State, M. W. (2014) 'Psychiatric Disorders: Diagnosis to Therapy', Cell, 157(1), pp. 201-214. doi: 10.1016/j.cell.2014.02.042.

Kyosseva, S. V. (2004) 'Differential expression of mitogen-activated protein kinases and immediate early genes fos and jun in thalamus in schizophrenia', Progress in NeuroPsychopharmacology and Biological Psychiatry, 28(6), pp. 997-1006. doi: 10.1016/j.pnpbp.2004.05.017.

Lacinova, L. et al. (2008) 'Cav1.2 calcium channels modulate the spiking pattern of hippocampal pyramidal cells', Life Sciences, 82(1-2), pp. 41-49. doi: 10.1016/j.lfs.2007.10.009.

Langova, V. et al. (2020) 'The Role of Zebrafish and Laboratory Rodents in Schizophrenia Research', Frontiers in Psychiatry, 11. doi: 10.3389/fpsyt.2020.00703.

Langwieser, N. et al. (2010) 'Homeostatic Switch in Hebbian Plasticity and Fear Learning after Sustained Loss of Cav1.2 Calcium Channels', Journal of Neuroscience, 30(25), pp. 8367-8375. doi: 10.1523/JNEUROSCI.4164-08.2010.

Lee, A. S. et al. (2012) 'Forebrain elimination of cacna1c mediates anxiety-like behavior in mice’, Molecular Psychiatry, 17(11), pp. 1054-1055. doi: 10.1038/mp.2012.71.

Lee, A. S. et al. (2016) 'The Neuropsychiatric Disease-Associated Gene cacna1c Mediates Survival of Young Hippocampal Neurons', eNeuro, 3(2). doi: 10.1523/ENEURO.0006-16.2016.

Lee, P. H. et al. (2019) 'Genomic Relationships, Novel Loci, and Pleiotropic Mechanisms across Eight Psychiatric Disorders’, Cell, 179(7), pp. 1469-1482.e11. doi: 10.1016/j.cell.2019.11.020.

Lee, S. H. et al. (2013) 'Genetic relationship between five psychiatric disorders estimated from genome-wide SNPs’, Nature Genetics, 45(9), pp. 984-994. doi: 10.1038/ng.2711.

Lein, E. S. et al. (2007) 'Genome-wide atlas of gene expression in the adult mouse brain', Nature, 445(7124), pp. 168-176. doi: 10.1038/nature05453.

Leitch, B. et al. (2009) 'Subcellular distribution of L-type calcium channel subtypes in rat hippocampal neurons', Neuroscience, 164(2), pp. 641-657. doi: 10.1016/j.neuroscience.2009.08.006.

Lewis, D. A., Hashimoto, T. and Volk, D. W. (2005) 'Cortical inhibitory neurons and schizophrenia', Nature Reviews Neuroscience, 6(4), pp. 312-324. doi: 10.1038/nrn1648. 
Li, J. et al. (2015) 'Schizophrenia Related Variants in CACNA1C also Confer Risk of Autism', PLoS ONE, 10(7). doi: 10.1371/journal.pone.0133247.

Lieschke, G. J. and Currie, P. D. (2007) 'Animal models of human disease: zebrafish swim into view’, Nature Reviews Genetics, 8(5), pp. 353-367. doi: 10.1038/nrg2091.

Lima-Ojeda, J. M. et al. (2019) 'Altered prepulse inhibition of the acoustic startle response in BDNF-deficient mice in a model of early postnatal hypoxia: implications for schizophrenia', European Archives of Psychiatry and Clinical Neuroscience, 269(4), pp. 439-447. doi: 10.1007/s00406-018-0882-6.

Lipscombe, D. and Andrade, A. (2015) 'Calcium Channel CaV $\alpha_{1}$ Splice Isoforms - Tissue Specificity and Drug Action', Current Molecular Pharmacology, 8(1), pp. 22-31. doi: $10.2174 / 1874467208666150507103215$.

Littleton, J. M., Little, H. J. and Whittington, M. A. (1990) 'Effects of dihydropyridine calcium channel antagonists in ethanol withdrawal; doses required, stereospecificity and actions of Bay K 8644', Psychopharmacology, 100(3), pp. 387-392. doi: 10.1007/BF02244612.

Liu, X. et al. (2016) Social Preference Deficits in Juvenile Zebrafish Induced by Early Chronic Exposure to Sodium Valproate | Behavioral Neuroscience. doi: 10.3389/fnbeh.2016.00201.

Liu, Y. et al. (2014) 'Cav1.2 and Cav1.3 L-type calcium channels regulate dopaminergic firing activity in the mouse ventral tegmental area', Journal of Neurophysiology, 112(5), pp. 11191130. doi: 10.1152/jn.00757.2013.

Lord, C. et al. (2018) 'Autism spectrum disorder', Lancet (London, England), 392(10146), pp. 508-520. doi: 10.1016/S0140-6736(18)31129-2.

Lord, C. et al. (2020) 'Autism spectrum disorder', Nature Reviews Disease Primers, 6(1), p. 5. doi: 10.1038/s41572-019-0138-4.

Maaswinkel, H., Zhu, L. and Weng, W. (2013) 'Assessing Social Engagement in Heterogeneous Groups of Zebrafish: A New Paradigm for Autism-Like Behavioral Responses’, PLOS ONE, 8(10), p. e75955. doi: 10.1371/journal.pone.0075955.

Magno, L. D. P. et al. (2015) 'Pharmacological study of the light/dark preference test in zebrafish (Danio rerio): Waterborne administration', Pharmacology Biochemistry and Behavior, 135, pp. 169-176. doi: 10.1016/j.pbb.2015.05.014.

Mäki, P. et al. (2005) 'Predictors of schizophrenia-a review', British Medical Bulletin, 7374(1), pp. 1-15. doi: 10.1093/bmb/ldh046.

Malhi, S. M. et al. (2014) 'Modulation of c-Fos and BDNF Protein Expression in Pentylenetetrazole-Kindled Mice following the Treatment with Novel Antiepileptic Compound HHL-6', BioMed Research International, 2014, pp. 1-9. doi: 10.1155/2014/876712. 
Manning, E. E. and van den Buuse, M. (2013) 'BDNF deficiency and young-adult methamphetamine induce sex-specific effects on prepulse inhibition regulation', Frontiers in Cellular Neuroscience, 7, p. 92. doi: 10.3389/fncel.2013.00092.

Marschallinger, J. et al. (2015) 'The L-type calcium channel Cav1.3 is required for proper hippocampal neurogenesis and cognitive functions', Cell Calcium, 58(6), pp. 606-616. doi: 10.1016/j.ceca.2015.09.007.

Maximino, C. et al. (2010) 'Measuring anxiety in zebrafish: A critical review', Behavioural Brain Research, 214(2), pp. 157-171. doi: 10.1016/j.bbr.2010.05.031.

Miller, N. and Gerlai, R. (2007) 'Quantification of shoaling behaviour in zebrafish (Danio rerio)', Behavioural Brain Research, 184(2), pp. 157-166. doi: 10.1016/j.bbr.2007.07.007.

Miller, N. and Gerlai, R. (2012) 'From Schooling to Shoaling: Patterns of Collective Motion in Zebrafish (Danio rerio)', PLoS ONE. Edited by G. G. de Polavieja, 7(11), p. e48865. doi: 10.1371/journal.pone.0048865.

Moon, A. L. et al. (2018) 'CACNA1C: Association With Psychiatric Disorders, Behavior, and Neurogenesis', Schizophrenia Bulletin, 44(5), pp. 958-965. doi: 10.1093/schbul/sby096.

Moosmang, S. et al. (2005) 'Role of Hippocampal Cav1.2 Ca2+ Channels in NMDA ReceptorIndependent Synaptic Plasticity and Spatial Memory', Journal of Neuroscience, 25(43), pp. 9883-9892. doi: 10.1523/JNEUROSCI.1531-05.2005.

Moran, L. V. and Hong, L. E. (2011) 'High vs Low Frequency Neural Oscillations in Schizophrenia', Schizophrenia Bulletin, 37(4), pp. 659-663. doi: 10.1093/schbul/sbr056.

Mullins, N. et al. (2021) 'Genome-wide association study of more than 40,000 bipolar disorder cases provides new insights into the underlying biology', Nature Genetics, pp. 1-13. doi: 10.1038/s41588-021-00857-4.

Napolitano, C. and Antzelevitch, C. (2011) 'Phenotypical manifestations of mutations in the genes encoding subunits of the cardiac voltage-dependent L-type calcium channel', Circulation research, 108(5), pp. 607-618. doi: 10.1161/CIRCRESAHA.110.224279.

N'Gouemo, P. et al. (2015) 'Alcohol Withdrawal-Induced Seizure Susceptibility is Associated with an Upregulation of CaV1.3 Channels in the Rat Inferior Colliculus', International Journal of Neuropsychopharmacology, 18(7), pp. pyu123-pyu123. doi: 10.1093/ijnp/pyu123.

N'Gouemo, P. and Morad, M. (2003) 'Ethanol withdrawal seizure susceptibility is associated with upregulation of L- and P-type Ca2+ channel currents in rat inferior colliculus neurons', Neuropharmacology, 45(3), pp. 429-437. doi: 10.1016/S0028-3908(03)00191-6.

Nguyen, T.-M. et al. (2019) 'Identifying significantly impacted pathways: a comprehensive review and assessment', Genome Biology, 20(1), p. 203. doi: 10.1186/s13059-019-1790-4. 
Nicolson, T. et al. (1998) 'Genetic Analysis of Vertebrate Sensory Hair Cell Mechanosensation: the Zebrafish Circler Mutants', Neuron, 20(2), pp. 271-283. doi: 10.1016/S08966273(00)80455-9.

Norton, W. H. J. (2012) 'Measuring Larval Zebrafish Behavior: Locomotion, Thigmotaxis, and Startle', in Kalueff, A. V. and Stewart, A. M. (eds) Zebrafish Protocols for Neurobehavioral Research. Totowa, NJ: Humana Press (Neuromethods), pp. 3-20. doi: 10.1007/978-1-61779597-8_1.

Notaras, M., Hill, R. and van den Buuse, M. (2015) 'The BDNF gene Val66Met polymorphism as a modifier of psychiatric disorder susceptibility: progress and controversy', Molecular Psychiatry, 20(8), pp. 916-930. doi: 10.1038/mp.2015.27.

Nyegaard, M. et al. (2010) 'CACNA1C (rs1006737) is associated with schizophrenia', Molecular Psychiatry, 15(2), pp. 119-121. doi: 10.1038/mp.2009.69.

Orellana-Paucar, A. M. et al. (2012) 'Anticonvulsant activity of bisabolene sesquiterpenoids of Curcuma longa in zebrafish and mouse seizure models', Epilepsy \& Behavior: E\&B, 24(1), pp. 14-22. doi: 10.1016/j.yebeh.2012.02.020.

O'Roak, B. J. et al. (2012) 'Sporadic autism exomes reveal a highly interconnected protein network of de novo mutations', Nature, 485(7397), pp. 246-250. doi: 10.1038/nature10989.

Owen, M. J., Sawa, A. and Mortensen, P. B. (2016) 'Schizophrenia', Lancet (London, England), 388(10039), pp. 86-97. doi: 10.1016/S0140-6736(15)01121-6.

Panula, P. et al. (2010) 'The comparative neuroanatomy and neurochemistry of zebrafish CNS systems of relevance to human neuropsychiatric diseases', Neurobiology of Disease, 40(1), pp. 46-57. doi: 10.1016/j.nbd.2010.05.010.

Pardiñas, A. F. et al. (2018) 'Common schizophrenia alleles are enriched in mutation-intolerant genes and in regions under strong background selection', Nature Genetics, 50(3), pp. 381-389. doi: 10.1038/s41588-018-0059-2.

Patel, K. R. et al. (2014) 'Schizophrenia: overview and treatment options', P \& T: A PeerReviewed Journal for Formulary Management, 39(9), pp. 638-645.

Pinggera, A. et al. (2015) 'CACNA1D De Novo Mutations in Autism Spectrum Disorders Activate Cav1.3 L-Type Calcium Channels', Biological Psychiatry, 77(9), pp. 816-822. doi: 10.1016/j.biopsych.2014.11.020.

Pinggera, A. et al. (2017) 'New gain-of-function mutation shows CACNA1D as recurrently mutated gene in autism spectrum disorders and epilepsy', Human Molecular Genetics, 26(15), pp. 2923-2932. doi: 10.1093/hmg/ddx175.

Pinggera, A. and Striessnig, J. (2016) 'Cav 1.3 (CACNA1D) L-type Ca2+ channel dysfunction in CNS disorders', The Journal of Physiology, 594(20), pp. 5839-5849. doi: 10.1113/JP270672. 
Pittenger, C. and Bloch, M. H. (2014) 'Pharmacological Treatment of Obsessive-Compulsive Disorder', Psychiatric Clinics of North America, 37(3), pp. 375-391. doi: 10.1016/j.psc.2014.05.006.

Platzer, J. et al. (2000) 'Congenital deafness and sinoatrial node dysfunction in mice lacking class D L-type Ca2+ channels’, Cell, 102(1), pp. 89-97. doi: 10.1016/s0092-8674(00)00013-1.

Plumbly, W. et al. (2019) 'L-type voltage-gated calcium channel regulation of in vitro human cortical neuronal networks', Scientific Reports, 9(1), p. 13810. doi: 10.1038/s41598-019-502269.

Pocklington, A. J. et al. (2015) 'Novel Findings from CNVs Implicate Inhibitory and Excitatory Signaling Complexes in Schizophrenia', Neuron, 86(5), pp. 1203-1214. doi:

10.1016/j.neuron.2015.04.022.

Prince, M. et al. (2007) 'No health without mental health', The Lancet, 370(9590), pp. 859-877. doi: 10.1016/S0140-6736(07)61238-0.

Prober, D. A. et al. (2006) 'Hypocretin/Orexin Overexpression Induces An Insomnia-Like Phenotype in Zebrafish', Journal of Neuroscience, 26(51), pp. 13400-13410. doi: 10.1523/JNEUROSCI.4332-06.2006.

Pucilowski, O. (1995) 'Isradipine Suppresses Amphetamine-Induced Conditioned Place Preference and Locomotor Stimulation in the Rat', Neuropsychopharmacology, 12(3), pp. 239244. doi: 10.1016/0893-133X(94)00080-J.

Purcell, S. M. et al. (2014) 'A polygenic burden of rare disruptive mutations in schizophrenia', Nature, 506(7487), pp. 185-190. doi: 10.1038/nature12975.

Ramachandran, K. V. et al. (2013) 'Calcium influx through L-type Cav1.2 $\mathrm{Ca}^{2+}$ channels regulates mandibular development', The Journal of Clinical Investigation, 123(4), pp. 16381646. doi: 10.1172/JCI66903.

Regier, D. A. et al. (2013) 'DSM-5 Field Trials in the United States and Canada, Part II: TestRetest Reliability of Selected Categorical Diagnoses', American Journal of Psychiatry, 170(1), pp. 59-70. doi: 10.1176/appi.ajp.2012.12070999.

Rehm, J. and Shield, K. D. (2019) 'Global Burden of Disease and the Impact of Mental and Addictive Disorders', Current Psychiatry Reports, 21(2), p. 10. doi: 10.1007/s11920-019-09970 .

Reimand, J. et al. (2019) 'Pathway enrichment analysis and visualization of omics data using g:Profiler, GSEA, Cytoscape and EnrichmentMap', Nature protocols, 14(2), pp. 482-517. doi: 10.1038/s41596-018-0103-9.

Rich, M. E. and Caldwell, H. K. (2015) 'A Role for Oxytocin in the Etiology and Treatment of Schizophrenia’, Frontiers in Endocrinology, 6. doi: 10.3389/fendo.2015.00090. 
Rihel, J. et al. (2010) 'Zebrafish behavioral profiling links drugs to biological targets and rest/wake regulation', Science (New York, N.Y.), 327(5963), pp. 348-351. doi: 10.1126/science. 1183090 .

Rihel, J. and Schier, A. F. (2012) 'Behavioral screening for neuroactive drugs in zebrafish', Developmental Neurobiology, 72(3), pp. 373-385. doi: 10.1002/dneu.20910.

Ripke, S. et al. (2014) 'Biological Insights From 108 Schizophrenia-Associated Genetic Loci', Nature, 511(7510), pp. 421-427. doi: 10.1038/nature13595.

Ross, J. et al. (2016) 'A Rare Variant in CACNA1D Segregates with 7 Bipolar I Disorder Cases in a Large Pedigree', Molecular Neuropsychiatry, 2(3), pp. 145-150. doi: 10.1159/000448041.

Rottbauer, W. et al. (2001) 'Growth and Function of the Embryonic Heart Depend upon the Cardiac-Specific L-Type Calcium Channel $\alpha 1$ Subunit', Developmental Cell, 1(2), pp. 265-275. doi: 10.1016/S1534-5807(01)00023-5.

Roussos, P. et al. (2014) 'A Role for Noncoding Variation in Schizophrenia', Cell Reports, 9(4), pp. 1417-1429. doi: 10.1016/j.celrep.2014.10.015.

Ruderfer, D. M. et al. (2018) 'Genomic Dissection of Bipolar Disorder and Schizophrenia, Including 28 Subphenotypes', Cell, 173(7), pp. 1705-1715.e16. doi: 10.1016/j.cell.2018.05.046.

Sakai, C., Ijaz, S. and Hoffman, E. J. (2018) 'Zebrafish Models of Neurodevelopmental Disorders: Past, Present, and Future', Frontiers in Molecular Neuroscience, 11. doi: 10.3389/fnmol.2018.00294.

Sakata, K. et al. (2009) 'Critical role of promoter IV-driven BDNF transcription in GABAergic transmission and synaptic plasticity in the prefrontal cortex', Proceedings of the National Academy of Sciences, 106(14), pp. 5942-5947. doi: 10.1073/pnas.0811431106.

Saliba, R. S. et al. (2009) 'Blocking L-type Voltage-gated Ca2+ Channels with Dihydropyridines Reduces $\gamma$-Aminobutyric Acid Type A Receptor Expression and Synaptic Inhibition', The Journal of Biological Chemistry, 284(47), pp. 32544-32550. doi: 10.1074/jbc.M109.040071.

Sanhueza, D. et al. (2009) 'Expression of voltage-activated calcium channels in the early zebrafish embryo’, Zygote, 17(2), pp. 131-135. doi: 10.1017/S0967199408005108.

Schierberl, K. et al. (2011) 'Cav1.2 L-Type Ca2+ Channels Mediate Cocaine-Induced GluA1 Trafficking in the Nucleus Accumbens, a Long-Term Adaptation Dependent on Ventral Tegmental Area Cav1.3 Channels', Journal of Neuroscience, 31(38), pp. 13562-13575. doi: 10.1523/JNEUROSCI.2315-11.2011.

Schnörr, S. et al. (2012) Measuring thigmotaxis in larval zebrafish - ScienceDirect. Available at: https://www.sciencedirect.com/science/article/pii/S0166432811008758?via\%3Dihub (Accessed: 5 December 2019). 
Scholl, U. I. et al. (2013) 'Somatic and germline CACNA1D calcium channel mutations in aldosterone-producing adenomas and primary aldosteronism', Nature Genetics, 45(9), pp. 10501054. doi: 10.1038/ng.2695.

Schubert, J. et al. (2014) 'Mutations in STX1B, encoding a presynaptic protein, cause feverassociated epilepsy syndromes', Nature Genetics, 46(12), pp. 1327-1332. doi: 10.1038/ng.3130.

Seibt, K. J. et al. (2010) 'Antipsychotic drugs prevent the motor hyperactivity induced by psychotomimetic MK-801 in zebrafish (Danio rerio)', Behavioural Brain Research, 214(2), pp. 417-422. doi: 10.1016/j.bbr.2010.06.014.

Seibt, K. J. et al. (2011) 'Antipsychotic drugs reverse MK-801-induced cognitive and social interaction deficits in zebrafish (Danio rerio)', Behavioural Brain Research, 224(1), pp. 135139. doi: 10.1016/j.bbr.2011.05.034.

Seisenberger, C. et al. (2000) 'Functional Embryonic Cardiomyocytes after Disruption of the Ltype $\alpha_{1 \mathrm{C}}(\mathrm{Ca}, 1.2)$ Calcium Channel Gene in the Mouse', Journal of Biological Chemistry, 275(50), pp. 39193-39199. doi: 10.1074/jbc.M006467200.

Shibasaki, M., Kurokawa, K. and Ohkuma, S. (2010) 'Upregulation of L-type Ca 1 channels in the development of psychological dependence', Synapse, 64(6), pp. 440-444. doi: 10.1002/syn.20745.

Sidi, S. et al. (2004) 'gemini Encodes a Zebrafish L-Type Calcium Channel That Localizes at Sensory Hair Cell Ribbon Synapses', Journal of Neuroscience, 24(17), pp. 4213-4223. doi: 10.1523/JNEUROSCI.0223-04.2004.

Sigitova, E. et al. (2017) 'Biological hypotheses and biomarkers of bipolar disorder: Hypotheses of bipolar disorder', Psychiatry and Clinical Neurosciences, 71(2), pp. 77-103. doi: 10.1111/pen.12476.

Sklar, P. et al. (2008) 'Whole-genome association study of bipolar disorder', Molecular Psychiatry, 13(6), pp. 558-569. doi: 10.1038/sj.mp.4002151.

Smith, F. A. (2008) 'Laboratory Tests and Diagnostic Procedures', in Massachusetts General Hospital Comprehensive Clinical Psychiatry. Elsevier, pp. 27-47. doi: 10.1016/B978-0-32304743-2.50005-6.

Smoller, J. W. (2013) 'Disorders and borders: Psychiatric genetics and nosology', American Journal of Medical Genetics Part B: Neuropsychiatric Genetics, 162(7), pp. 559-578. doi: 10.1002/ajmg.b.32174.

Smoller, J. W. et al. (2019) 'Psychiatric genetics and the structure of psychopathology', Molecular Psychiatry, 24(3), pp. 409-420. doi: 10.1038/s41380-017-0010-4.

Spitsbergen, J. M. and Kent, M. L. (2003) 'The State of the Art of the Zebrafish Model for Toxicology and Toxicologic Pathology Research-Advantages and Current Limitations', Toxicologic Pathology, 31(1_suppl), pp. 62-87. doi: 10.1080/01926230390174959. 
Splawski, I. et al. (2004) 'CaV1.2 Calcium Channel Dysfunction Causes a Multisystem Disorder Including Arrhythmia and Autism', Cell, 119(1), pp. 19-31. doi: 10.1016/j.cell.2004.09.011.

Splawski, I. et al. (2005) 'Severe arrhythmia disorder caused by cardiac L-type calcium channel mutations', Proceedings of the National Academy of Sciences, 102(23), pp. 8089-8096. doi: 10.1073/pnas.0502506102.

Sponheim, S. R. et al. (1994) 'Resting EEG in first-episode and chronic schizophrenia', Psychophysiology, 31(1), pp. 37-43. doi: 10.1111/j.1469-8986.1994.tb01023.x.

Stewart, A. M. et al. (2014) 'Zebrafish models for translational neuroscience research: from tank to bedside', Trends in neurosciences, 37(5), pp. 264-278. doi: 10.1016/j.tins.2014.02.011.

Sullivan, P. F. and Geschwind, D. H. (2019) 'Defining the Genetic, Genomic, Cellular, and Diagnostic Architectures of Psychiatric Disorders’, Cell, 177(1), pp. 162-183. doi:

10.1016/j.cell.2019.01.015.

Sykes, L. et al. (2019) 'Genetic Variation in the Psychiatric Risk Gene CACNA1C Modulates Reversal Learning Across Species', Schizophrenia Bulletin, 45(5), pp. 1024-1032. doi: $10.1093 / \mathrm{schbul} / \mathrm{sby} 146$.

Tabuchi, A. et al. (2000) 'Differential Activation of Brain-derived Neurotrophic Factor Gene Promoters I and III by Ca2+ Signals Evoked vial-type Voltage-dependent andN-Methyl-daspartate Receptor Ca2+Channels', Journal of Biological Chemistry, 275(23), pp. 17269-17275. doi: 10.1074/jbc.M909538199.

Tang, Z. Z. et al. (2004) 'Transcript scanning reveals novel and extensive splice variations in human 1-type voltage-gated calcium channel, Cav1.2 alpha1 subunit', The Journal of Biological Chemistry, 279(43), pp. 44335-44343. doi: 10.1074/jbc.M407023200.

Terrillion, C. E. et al. (2017) 'Reduced levels of Cacna1c attenuate mesolimbic dopamine system function', Genes, Brain and Behavior, 16(5), pp. 495-505. doi: https://doi.org/10.1111/gbb.12371.

Tesli, M. et al. (2013) 'CACNA1C Risk Variant and Amygdala Activity in Bipolar Disorder, Schizophrenia and Healthy Controls', PLoS ONE, 8(2). doi: 10.1371/journal.pone.0056970.

The Brainstorm Consortium et al. (2018) 'Analysis of shared heritability in common disorders of the brain', Science, 360(6395), p. eaap8757. doi: 10.1126/science.aap8757.

The Network and Pathway Analysis Subgroup of the Psychiatric Genomic Consortium (2015) 'Psychiatric genome-wide association study analyses implicate neuronal, immune and histone pathways', Nature neuroscience, 18(2), pp. 199-209. doi: 10.1038/nn.3922.

The Wellcome Trust Case Control Consortium (2007) 'Genome-wide association study of 14,000 cases of seven common diseases and 3,000 shared controls', Nature, 447(7145), pp. 661678. doi: 10.1038/nature05911. 
Thyme, S. B. et al. (2019) 'Phenotypic Landscape of Schizophrenia-Associated Genes Defines Candidates and Their Shared Functions', Cell, 177(2), pp. 478-491.e20. doi: 10.1016/j.cell.2019.01.048.

Tigaret, C. M. et al. (2021) 'Neurotrophin receptor activation rescues cognitive and synaptic abnormalities caused by hemizygosity of the psychiatric risk gene Cacna1c', Molecular Psychiatry. doi: 10.1038/s41380-020-01001-0.

Tippens, A. L. et al. (2008) 'Ultrastructural evidence for pre- and postsynaptic localization of Cav1.2 L-type Ca2+ channels in the rat hippocampus', Journal of Comparative Neurology, 506(4), pp. 569-583. doi: https://doi.org/10.1002/cne.21567.

Tiraboschi, E. et al. (2020) 'New insights into the early mechanisms of epileptogenesis in a zebrafish model of Dravet syndrome', Epilepsia, 61(3), pp. 549-560. doi: 10.1111/epi.16456.

Todorova, V. K., Elbein, A. D. and Kyosseva, S. V. (2003) 'Increased Expression of c-Jun Transcription Factor in Cerebellar Vermis of Patients with Schizophrenia', Neuropsychopharmacology, 28(8), pp. 1506-1514. doi: 10.1038/sj.npp.1300211.

Uher, R. and Zwicker, A. (2017) 'Etiology in psychiatry: embracing the reality of poly-geneenvironmental causation of mental illness', World Psychiatry, 16(2), pp. 121-129. doi: $10.1002 /$ wps. 20436 .

Uhlen, M. et al. (2015) 'Tissue-based map of the human proteome', Science, 347(6220), pp. 1260419-1260419. doi: 10.1126/science.1260419.

Uhlhaas, P. J. et al. (2008) 'The Role of Oscillations and Synchrony in Cortical Networks and Their Putative Relevance for the Pathophysiology of Schizophrenia', Schizophrenia Bulletin, 34(5), pp. 927-943. doi: 10.1093/schbul/sbn062.

Uhlhaas, P. J. and Singer, W. (2010) 'Abnormal neural oscillations and synchrony in schizophrenia', Nature Reviews Neuroscience, 11(2), pp. 100-113. doi: 10.1038/nrn2774.

Uhlhaas, P. J. and Singer, W. (2013) 'High-frequency oscillations and the neurobiology of schizophrenia', Dialogues in Clinical Neuroscience, 15(3), pp. 301-313. doi:

10.31887/DCNS.2013.15.3/puhlhaas.

Väisänen, J. et al. (2004) 'Effects of NMDA-Receptor Antagonist Treatment on c-fos Expression in Rat Brain Areas Implicated in Schizophrenia', Cellular and Molecular Neurobiology, 24(6), pp. 769-780. doi: 10.1007/s10571-004-6918-7.

Velazquez, F. N., Caputto, B. L. and Boussin, F. D. (2015) 'c-Fos importance for brain development', Aging, 7(12), pp. 1028-1029. doi: 10.18632/aging.100862.

Vieta, E. et al. (2018) 'Bipolar disorders', Nature Reviews Disease Primers, 4(1), p. 18008. doi: 10.1038/nrdp.2018.8. 
Viveros, M. P. et al. (1996) 'Effects of nimodipine and nifedipine upon behavior and regional brain monoamines in the rat', Psychopharmacology, 127(2), pp. 123-132. doi: 10.1007/BF02805985.

Vorstman, J. A. S. et al. (2017) 'Autism genetics: opportunities and challenges for clinical translation’, Nature Reviews Genetics, 18(6), pp. 362-376. doi: 10.1038/nrg.2017.4.

Vorstman, J. A. S. and Burbach, J. P. H. (2014) 'Autism and Schizophrenia: Genetic and Phenotypic Relationships', in Patel, V. B., Preedy, V. R., and Martin, C. R. (eds) Comprehensive Guide to Autism. New York, NY: Springer New York, pp. 1645-1662. doi: 10.1007/978-1-46144788-7_96.

Wellcome Trust Case Control Consortium et al. (2010) 'The bipolar disorder risk allele at CACNA1C also confers risk of recurrent major depression and of schizophrenia', Molecular Psychiatry, 15(10), pp. 1016-1022. doi: 10.1038/mp.2009.49.

Wemhöner, K. et al. (2015) 'Gain-of-function mutations in the calcium channel CACNA1C (Cav1.2) cause non-syndromic long-QT but not Timothy syndrome', Journal of Molecular and Cellular Cardiology, 80, pp. 186-195. doi: 10.1016/j.yjmcc.2015.01.002.

West, A. E., Griffith, E. C. and Greenberg, M. E. (2002) 'Regulation of transcription factors by neuronal activity', Nature Reviews Neuroscience, 3(12), pp. 921-931. doi: 10.1038/nrn987.

Westenbroek, R. E., Hoskins, L. and Catterall, W. A. (1998) 'Localization of Ca2+ Channel Subtypes on Rat Spinal Motor Neurons, Interneurons, and Nerve Terminals', Journal of Neuroscience, 18(16), pp. 6319-6330. doi: 10.1523/JNEUROSCI.18-16-06319.1998.

White, J. A. et al. (2008) 'Conditional forebrain deletion of the L-type calcium channel CaV1.2 disrupts remote spatial memories in mice', Learning \& Memory, 15(1), pp. 1-5. doi: 10.1101/lm.773208.

Widge, A. S. and Moritz, C. T. (2016) 'Closed-Loop Stimulation in Emotional Circuits for Neuro-Psychiatric Disorders', in Closed Loop Neuroscience. Elsevier, pp. 229-239. doi: 10.1016/B978-0-12-802452-2.00017-2.

Winchester, C. L., Pratt, J. A. and Morris, B. J. (2014) 'Risk genes for schizophrenia: translational opportunities for drug discovery', Pharmacology \& Therapeutics, 143(1), pp. 3450. doi: 10.1016/j.pharmthera.2014.02.003.

Wiser, O. et al. (1999) 'The voltage sensitive Lc-type Ca2+ channel is functionally coupled to the exocytotic machinery', Proceedings of the National Academy of Sciences, 96(1), pp. 248253. doi: 10.1073/pnas.96.1.248.

Wiser, O., Bennett, M. K. and Atlas, D. (1996) 'Functional interaction of syntaxin and SNAP-25 with voltage-sensitive L- and N-type Ca2+ channels.', The EMBO Journal, 15(16), pp. 41004110. doi: 10.1002/j.1460-2075.1996.tb00785.x. 
Woods, I. G. et al. (2014) 'Neuropeptidergic Signaling Partitions Arousal Behaviors in Zebrafish', The Journal of Neuroscience, 34(9), pp. 3142-3160. doi:

10.1523/JNEUROSCI.3529-13.2014.

Wullimann, M. F. and Mueller, T. (2004) 'Teleostean and mammalian forebrains contrasted: Evidence from genes to behavior', Journal of Comparative Neurology, 475(2), pp. 143-162. doi: 10.1002/cne.20183.

$\mathrm{Xu}, \mathrm{W}$. and Lipscombe, D. (2001) 'Neuronal CaV1.3a1 L-Type Channels Activate at Relatively Hyperpolarized Membrane Potentials and Are Incompletely Inhibited by Dihydropyridines', Journal of Neuroscience, 21(16), pp. 5944-5951. doi: 10.1523/JNEUROSCI.21-16-05944.2001.

Yenkoyan, K. et al. (2017) 'Advances in understanding the pathophysiology of autism spectrum disorders', Behavioural Brain Research, 331, pp. 92-101. doi: 10.1016/j.bbr.2017.04.038.

Yoshimizu, T. et al. (2015) 'Functional implications of a psychiatric risk variant within CACNA1C in induced human neurons', Molecular psychiatry, 20(2), pp. 162-169. doi: 10.1038/mp.2014.143.

Zamponi, G. W. et al. (2015) 'The Physiology, Pathology, and Pharmacology of Voltage-Gated Calcium Channels and Their Future Therapeutic Potential', Pharmacological Reviews, 67(4), pp. 821-870. doi: 10.1124/pr.114.009654.

Zamponi, G. W. (2016) 'Targeting voltage-gated calcium channels in neurological and psychiatric diseases', Nature Reviews Drug Discovery, 15(1), pp. 19-34. doi: 10.1038/nrd.2015.5.

Zhang, J. et al. (2002) 'c-fos regulates neuronal excitability and survival', Nature Genetics, 30(4), pp. 416-420. doi: 10.1038/ng859.

Zuccotti, A. et al. (2011) 'Structural and functional differences between L-type calcium channels: crucial issues for future selective targeting', Trends in Pharmacological Sciences, 32(6), pp. 366-375. doi: 10.1016/j.tips.2011.02.012. 


\section{APPENDIX}

\section{CURRICULUM VITAE}

\section{PERSONAL INFORMATION}

Name: Nancy Saana Banono

Born: 04.11.91

Nationality: Ghana

Civil status: Married

Mobile: (+47) 48342688

Email address: nancy.banono@gmail.com

\section{EDUCATION AND TRAINING}

Doctor of Philosophy, Chemical Neuroscience, 2017 - date, University of Oslo, Oslo (Norway)

Master of Science, Neuroscience, 2015 - 2017, Norwegian University of Science and Technology (NTNU), Trondheim (Norway)

Bachelor of Science (Hons), Human Biology, 2010 - 2014, University of Cape Coast, Cape Coast (Ghana)

\section{WORK EXPERIENCE}

Doctoral Research Training, August 2017 till date, Centre for Molecular Medicine Norway (NCMM), Oslo (Norway)

Industrial Intern, September 2020 - December 2020, Theracule AS, Oslo (Norway)

Volunteer, April 2018 - May 2019, Emergency shelter for the homeless, Oslo Red Cross, Oslo (Norway)

Staff Engineer, June 2016 - June 2017, Kavli Institute for Systems Neuroscience Centre for Neural Computation, NTNU, Trondheim (Norway)

Undergraduate Teaching Assistant, October 2014 - August 2015, Department of Biomedical and Forensic Sciences. University of Cape Coast, Cape Coast (Ghana)

Learner Guide, October 2014 - June 2015, Campaign for Female Education (Camfed) - Ghana Clinical Laboratory Intern, July 2012 - August 2012, Tamale Teaching Hospital, Tamale (Ghana)

Pupil Teacher, September 2009 - March 2010, High Standards Girls' Junior High School, Tamale (Ghana)

\section{SUPERVISION}

I have supervised one master's student (from 2020/2021 academic year) and two undergraduate students (one in 2019 and the other in 2020). 


\section{EXPERIMENTAL SKILLS AND EXPERTISE}

- $\quad$ CRISPR/Cas9 mutagenesis

- Microinjection of zebrafish embryos

- Zebrafish embryonic development

- Pharmacological profiling of zebrafish

- Behavioural studies of zebrafish

- Molecular biology i.e., PCR, cloning, western blotting

- Wholemount in situ hybridization analysis

- Local field potential recordings

\section{FELLOWSHIPS AND AWARDS}

2020 Centre for Digital Life Norway Industry Internship Grant

Funding to undertake a three-month internship at Theracule AS, Oslo - Norway.

2015 Quota Scheme

Two-year scholarship to study my Masters at NTNU, Trondheim - Norway, 2015-2017.

2014 German-Ghanaian Study and Exchange Program, Münster - Tamale

Fellowship to study in Münster, Germany, summer 2014.

\section{SCIENTIFIC TALKS}

- Characterisation of Zebrafish cacna1c Mutant- a Model for Schizophrenia (December 10, 2020). Neuronus 2020 IBRO Neuroscience Forum hosted by online by Jagiellonian University, Krakow-Poland; 8 - 11 December, 2020

- Zebrafish larvae carrying a mutation in cacna1d display schizophrenia-like behaviours (September 24, 2020). Nordic EMBL Partnership Virtual Meeting, Umeå-2020.

- The utility of zebrafish (Danio rerio) in neuroscience research (May 19, 2020). Digital Stunt Lunch, SINTEF-Digital, Oslo.

- Phenotypic characterisation of larval zebrafish with mutations in the cacna1c gene (December 16, 2019). DigiBrain Symposium, NHO Møtesenter - Oslo.

\section{POSTER PRESENTATIONS AT CONFERENCES}

- Banono, Nancy Saana; Gawel, Kinga Aurelia; De Witte, Linus \& Esguerra, Camila V. (2020). Zebrafish larvae carrying a mutation in cacna1d display schizophrenia-like behaviours.

Nordic EMBL Partnership Virtual Meeting, Umeå-2020

- Banono, Nancy Saana DigiBrain Consortium \& Esguerra, Camila V. (2020). Phenotypic analysis and small-molecule drug screening in genetic zebrafish models of schizophrenia. 2nd International Fishmed Conference, Warsaw, Poland 2018. 


\section{PUBLICATIONS}

\section{Scientific Publications}

- Banono, Nancy Saana; Gawel, Kinga Aurelia; De Witte, Linus \& Esguerra, Camila (2020). Zebrafish larvae carrying a splice variant mutation in cacna1d: a new model for schizophrenia-like behaviours? Molecular Neurobiology. ISSN 0893-7648. doi: 10.1007/s12035-020-02160-5

- Banono, Nancy Saana \& Esguerra, Camila (2020). Pharmacological validation of the prepulse inhibition of startle response in larval zebrafish using a commercial automated system and software. Journal of Visualized Experiments. ISSN 1940-087X. 161. doi: 10.3791/61423

- Gawel, Kinga Aurelia; Kukula-Koch, Wirginia; Nieoczym, Dorota; Stępnik, Katarzyna; van der Ent, Wietske; Banono, Nancy Saana; Tarabasz, Dominik; Turski, Waldemar A. \& Esguerra, Camila (2020). The Influence of Palmatine Isolated from Berberis sibirica Radix on Pentylenetetrazole-Induced Seizures in Zebrafish. Cells. ISSN 2073-4409. 9(5). doi: 10.3390/cells9051233

- Gawel, Kinga Aurelia; Banono, Nancy Saana; Michalak, Agnieszka \& Esguerra, Camila Vicencio (2019). A critical review of zebrafish schizophrenia models: Time for validation. Neuroscience and Biobehavioral Reviews. ISSN 0149-7634. 107, s 6- 22. doi: 10.1016 / j.neubiorev.2019.08.001

- Banono, Nancy Saana (2017). "Studying Autosomal Dominant Nocturnal Frontal Lobe Epilepsy (ADNFLE) in chrna4 mutant zebrafish". Master thesis

- Banono, Nancy Saana (2014). "The effect of crude aqueous root extract of Haematostaphis barteri on humoral immune response of ICR mice". Undergraduate thesis

\section{Other Publications}

- Banono, Nancy Saana (2021). When do I tell my supervisor? Science. 372 (6540), pp. 430. doi: $10.1126 /$ science.372.6540.430

- Juskewitz, Eric; Heck, Kathleen Anne \& Banono, Nancy Saana (2021). Why industry internships can be your 'golden ticket' to a prosperous career. Nature. doi: 10.1038/d41586-021-00730-8

\section{ADDITIONAL PROFESSIONAL MERITS}

- Participated in academic video production titled "zebrafish as a model organism" for the Developmental Biology students (BIOS3601/4601) at UiO. Zebrafish Facility, NCMM. October 2020. https://www.youtube.com/watch?v=Tu4gYDBzEGA\&feature=youtu.be

- Member of the Organizing Committee, Young Investigator Meeting during the $9^{\text {th }}$ Nordic EMBL Network Meeting at Soria Moria Hotel, Oslo from 11 - 14 September 2018.

- Participated in filming the Zebrafish $360^{\circ}$ video series as part of a zebrafish e-book led by Peter Alestrøm of NMBU. https://www.zebrafishlab.net/kopi-av-vr-meny. January 2018. 
- Member of the Adhoc Social Committee for the NCMM retreat at Larvik from 6-7 November, 2017

\section{MEMBERSHIPS}

- Young African Leaders Initiative (YALI) Network

- Campaign for Female Education Association (CAMA) - Ghana Network

- Norwegian Red Cross

- Digital Life Norway Research School

- Norwegian Research School of Neuroscience

- Norwegian PhD School of Pharmacy 


\section{PAPERS}




\section{Paper I}

\section{A critical review of zebrafish schizophrenia models: Time for validation?}

Gawel, Kinga Aurelia; Banono, Nancy Saana; Michalak, Agnieszka \& Esguerra, Camila V.

Published in Neuroscience and Biobehavioral Reviews, August 2019, volume 107, issue 2, pp. 6-22. DOI: 10.1016/j.neubiorev.2019.08.001 

Review article

\title{
A critical review of zebrafish schizophrenia models: Time for validation?
}

\author{
Kinga Gawel ${ }^{\mathrm{a}, \mathrm{b}, *}$, Nancy Saana Banono ${ }^{\mathrm{a}}$, Agnieszka Michalak ${ }^{\mathrm{c}}$, Camila V. Esguerra ${ }^{\mathrm{a}, \mathrm{d}, *}$ \\ ${ }^{a}$ Chemical Neuroscience Group, Centre for Molecular Medicine Norway, University of Oslo, Gaustadalléen 21, 0349, Oslo, Norway \\ ${ }^{\mathrm{b}}$ Department of Experimental and Clinical Pharmacology, Medical University of Lublin, Jaczewskiego St. 8b, 20-090, Lublin, Poland \\ ${ }^{\mathrm{c}}$ Department of Pharmacology and Pharmacodynamics, Medical University of Lublin, Chodzki St. 4A, 20-093, Lublin, Poland \\ d Department of Pharmacy, University of Oslo, Oslo, Norway
}

\section{A R T I C L E I N F O}

\section{Keywords:}

Zebrafish

Schizophrenia

Models

Risk genes

Drugs

Validation

\begin{abstract}
A B S T R A C T
Schizophrenia is a mental disorder that affects $1 \%$ of the population worldwide and is manifested as a broad spectrum of symptoms, from hallucinations to memory impairment. It is believed that genetic and/or environmental factors may contribute to the occurrence of this disease. Recently, the zebrafish has emerged as a valuable and attractive model for various neurological disorders including schizophrenia. In this review, we describe current pharmacological models of schizophrenia with special emphasis on providing insights into the pros and cons of using zebrafish as a behavioural model of this disease. Moreover, we highlight the advantages and utility of using zebrafish for elucidating the genetic mechanisms underlying this psychiatric disorder. We believe that the zebrafish has high potential also in the area of precision medicine and may complement the development of therapeutics, especially for pharmacoresistant patients.
\end{abstract}

\section{Introduction}

Schizophrenia is a neuropsychiatric disorder that affects $1 \%$ of the global population and is among the leading causes of disability worldwide. Schizophrenia is characterized by a large diversity of symptoms from positive (e.g. delusions, hallucinations, stereotypic behaviour) and negative (e.g. alogia, social withdrawal, affective flattening) to cognitive (e.g. attention deficits, cognitive deficits, memory impairment). The heritability estimate is $80 \%$ based on data from identical twin studies (Winchester et al., 2014). A number of schizophrenia susceptibility genes have been identified by genome-wide association studies (GWAS) (Cross-disorder group of Psychiatric Genomics Consortium, 2013; Schizophrenia Working Group of the Psychiatric Genomics Consortium, 2014) while copy number variants (CNVs) show that the disease is polygenic in nature (Purcell et al., 2014). An individual's genetic predisposition coupled with environmental factors cumulatively contributes to the aetiology of schizophrenia (Patel et al., 2014).

Since the revolutionary use of the zebrafish as a model organism by George Streisinger in the 1960s, mainly for developmental and toxicological studies, there has been a tremendous increase in the use of this organism in modelling human diseases (Lieschke and Currie, 2007). The zebrafish is fast emerging as an ideal model to study psychiatric diseases because of the numerous advantages it possesses (Kalueff et al.,
2014). The genome of zebrafish has been fully sequenced revealing about $70 \%$ gene homology with humans and a vast number of molecular genetic tools which are useful for easy manipulation of the zebrafish genome are now available (Howe et al., 2013). Another important advantage of zebrafish is that they are highly fecund and can produce between 50-300 eggs weekly. The eggs develop externally and are small (ca. $4 \mathrm{~mm}$ at 5 days post-fertilization, dpf) (Kimmel et al., 1995). This makes it easy to array embryos and larvae in 96-well microtiter plates for high-throughput drug screening (Kalueff et al., 2014). Also, zebrafish transparency at early stages of development allows for non-invasive in vivo calcium imaging using transgenic reporter lines.

\subsection{Brain similarity}

Zebrafish share a similar brain architecture with humans. The forebrain, midbrain, hindbrain and spinal cord are present in zebrafish. The optic tectum, thalamus and cerebellum of zebrafish are homologous to that of humans (Guo, 2009). It is worth mentioning, however, that the zebrafish lacks a neocortex. Despite this, zebrafish are still capable of cognitive processing and complex decision making (Jarvis et al., 2005). Moreover, the optic tectum in zebrafish is more complex than its mammalian counterpart, the superior colliculus. It has therefore been postulated that the complexity of the tectum enables it to perform vision-related functions that were eventually taken over by the

\footnotetext{
*Corresponding authors at: Chemical Neuroscience Group, Centre for Molecular Medicine Norway, University of Oslo, Gaustadalléen 21, 0349, Oslo, Norway.

E-mail addresses: kingagawel@umlub.pl (K. Gawel), c.v.esguerra@ncmm.uio.no (C.V. Esguerra).
} 
mammalian neocortex. Another region, the pallium, is homologous to cortical structures, but with no distinct layering, except for a greater density of neural cell bodies near the surface (Mueller and Wullimann, 2015). The telencephalon of zebrafish develops through the process of eversion while that of mammals arises via the process of invagination (Wullimann and Mueller, 2004). These distinct processes result in differences in the gross anatomical structure of the brain. For example, the habenula, amygdala and hippocampus that are otherwise deep-seated within the mammalian brain have homologous structures in the zebrafish that lie in the most dorsal position of the telencephalon (Leung et al., 2013). Although these differences make direct comparisons complicated, it provides an opportunity in that these otherwise difficult to reach nuclei can easily be imaged and studied. In addition, zebrafish develop a blood-brain barrier (BBB) at $3 \mathrm{dpf}$, which becomes fully functional by $10 \mathrm{dpf}$. Before $10 \mathrm{dpf}$, the BBB is leaky, allowing smaller sized molecules such as sulfo-NHS-biotin to pass through it (Jeong et al., 2008; Fleming et al., 2013). This is relevant for drug screens aiming to identify neuromodulatory agents - meaning that up until 9 dpf it is possible to have drugs delivered through simple bathing of embryos and larvae in solution. Moreover, the zebrafish BBB shares structural and functional similarities with mammals such as the presence of tight junction proteins claudin- 5 and $\mathrm{ZO}-1$ already present at 3 $\mathrm{dpf}$ and the presence of the active transport protein ABCB1 at $8 \mathrm{dpf}$ (Jeong et al., 2008; Fleming et al., 2013). Furthermore, zebrafish has all the major neurotransmitters with their corresponding receptors, transporters and enzyme synthesis and metabolism pathways present in humans and rodents (Panula et al., 2010). These neurotransmitter pathways are conserved and zebrafish have been shown to respond to major classes of psychoactive drugs (Kokel and Peterson, 2008; Rihel et al., 2010).

\subsection{Genetic tools for zebrafish research}

The diverse range of forward and reverse genetic tools enable researchers to manipulate the zebrafish genome with relative ease and efficiency (see Fig. 1), in order to identify the contribution of a given gene variant to the development of disease (Kalueff et al., 2014). $\mathrm{N}$ ethyl- $N$-nitrosourea (ENU) is a chemical mutagen that leads to the introduction of random single point mutations. ENU is mainly used as a forward genetic tool and involves simply "bathing" adult male zebrafish in a tank of ENU solution. The treated ENU males are crossed with females to produce the F1 generation. The F1 generation are randomly crossed to propagate the mutations further within the resulting F2 generation. In the F3 generation, a portion of fish carrying homozygous recessive mutations will display a phenotype. These phenotypes are investigated and linkage analysis carried out to identify the actual mutation (for review, see Lawson and Wolfe, 2011). Antisense morpholino oligonucleotides (MO) are used to transiently knock-down gene expression by inhibiting mRNA splicing or translation. The efficacy period of MO target inhibition lasts a maximum of 5-6 days and results in variable knock-down due to the fact that target inhibition is not due to a stable genetic lesion (for review, see Bill et al., 2009). Established transgenesis methods using cell- and tissue-specific promoters or enhancers in zebrafish (Kawakami, 2005; Kawakami et al., 1998; Suster et al., 2009) enable (over)production of any given exogenous protein (e.g. fluorescent reporter protein, mutant protein) in specific cell types such as neurons, neuroglia, blood vessels, etc. (Rinkwitz et al., 2011). Nuclease-based genome editing tools such as zinc finger nucleases (ZFNs), transcription activator like effector nucleases (TALENs) and CRISPR/Cas9 are widely used in creating gene knock-out and knock-in lines (for review, see Sertori et al., 2016). This vast molecular toolbox and the genetic tractability of the zebrafish make it an ideal model organism for studying the role of disease susceptibility genes (see Fig. 1).

\subsection{Behaviour toolbox for zebrafish research}

Behaviorally, several neuro-phenotypic domains such as fear, anxiety, aggression, startle response, learning and memory and the sleepwake cycle, among others, have been modelled and characterized in zebrafish (Kalueff et al., 2014; Leung et al., 2013). The presence of these tools coupled with the sensitivity of zebrafish to the major classes of psychoactive drugs makes it an ideal candidate of choice for pharmacological manipulations and phenotypic drug screening (Kokel and Peterson, 2008). Furthermore, the zebrafish, like humans, are diurnal, meaning their visual acuity is well developed and simplifies observation during the photoperiod. Visual cues are relevant in learning and memory tasks and suggest that the zebrafish could be used as good models to investigate cognitive declines such as impaired learning and memory as seen in most neuropsychiatric diseases such as schizophrenia (Gerlai, 2016). Pre-pulse inhibition (PPI) is a form of sensorimotor gating in which the startle response is attenuated when a weaker non-startling stimulus is presented shortly before the startling stimulus (Bhandiwad et al., 2013). Impairment of PPI has been observed in schizophrenia as well as in a number of neurological diseases. Interestingly, antipsychotic drugs have been reported to reverse PPI deficits in schizophrenia patients as well as in animal models. This finding has made PPI a useful endophenotype for the genetic analysis of schizophrenia (Morris, 2009). Evidence has since been provided to support the fact that larval zebrafish also exhibit PPI as early as $3 \mathrm{dpf}$ (Bhandiwad et al., 2013; Burgess and Granato, 2007; reviewed by López-Schier, 2019) (for behavioral set up and data output see Fig. 2).

\subsection{Monitoring brain activity in zebrafish}

The optical transparency of the zebrafish during larval and juvenile stages, its small brain size i.e. $1.5 \mathrm{~mm}$ and $4.5 \mathrm{~mm}$ long in larval and adult zebrafish respectively (Wullimann et al., 1996a, 1996b, 1996c, $1996 \mathrm{~d}, 1996 \mathrm{e}, 1996 \mathrm{f}$ ) and its lower total number of neurons (about $10^{5}$ and $10^{7}$ in larval and adult zebrafish respectively) (Hill et al., 2003; Hinsch and Zupanc, 2007) makes the zebrafish useful for studying the structure and function of neural circuits when coupled with genetic fluorescence reporters (Friedrich et al., 2013). Optical techniques, such as confocal or multiphoton imaging have been developed to enable simultaneous monitoring of up to a thousand neuronal activities in vivo (Rinkwitz et al., 2011; Wyatt et al., 2015). This provides deeper insights into the structure and function of the neuronal circuits in both the healthy and diseased brain and may provide new windows of opportunity in developing therapies for the numerous neurological diseases including schizophrenia.

in vivo confocal calcium imaging has been used to study the neural basis of the escape response and habituation in zebrafish larvae (Takahashi et al., 2002, 2017; Kohashi and Oda, 2008a). Mauthner cells are giant cells in the reticulospinal neurons responsible for the fast escape response (C-bend) in zebrafish (Takahashi et al., 2002). Takahashi et al. bulk loaded dextran, a calcium sensitive dye retrogradely into Mauthner cells. With the use of confocal microscopy and patch clamp analysis, they identified the inhibitory network that regulates Mauthner cell excitability, while another study (Gahtan et al., 2002) investigated the contribution of non-Mauthner cells to the zebrafish escape response using Oregon-green dextran, a fluorescent calcium indicator. Takahashi et al. (2017) showed that the firing of Mauthner cells was linked to the onset of slow latency C-bends (SLCs). In cases where the brain region governing a particular behaviour or the impact of a gene lesion on neural activity is not yet known, it is possible to use two photon microscopy to capture whole-brain network activity (Light and Jontes, 2019; Wyatt et al., 2015). For example, by using in vivo two photon calcium imaging, researchers reported enhanced clustering and an altered developmental trajectory of network assembly in pcdh19 zebrafish mutants when compared to wild type counterparts (Light and Jontes, 2019). 
A

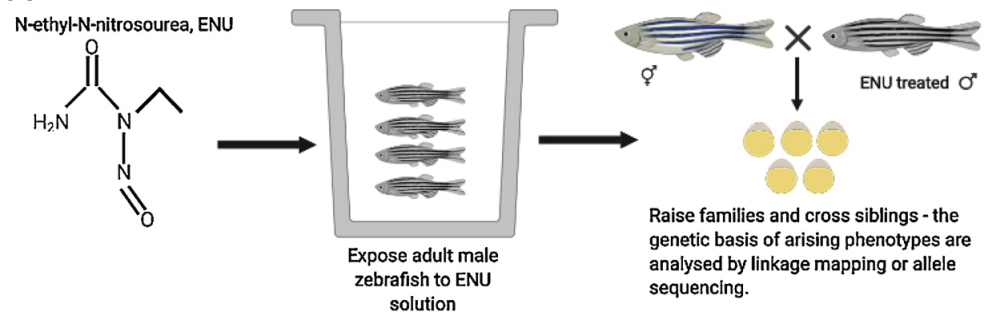

B
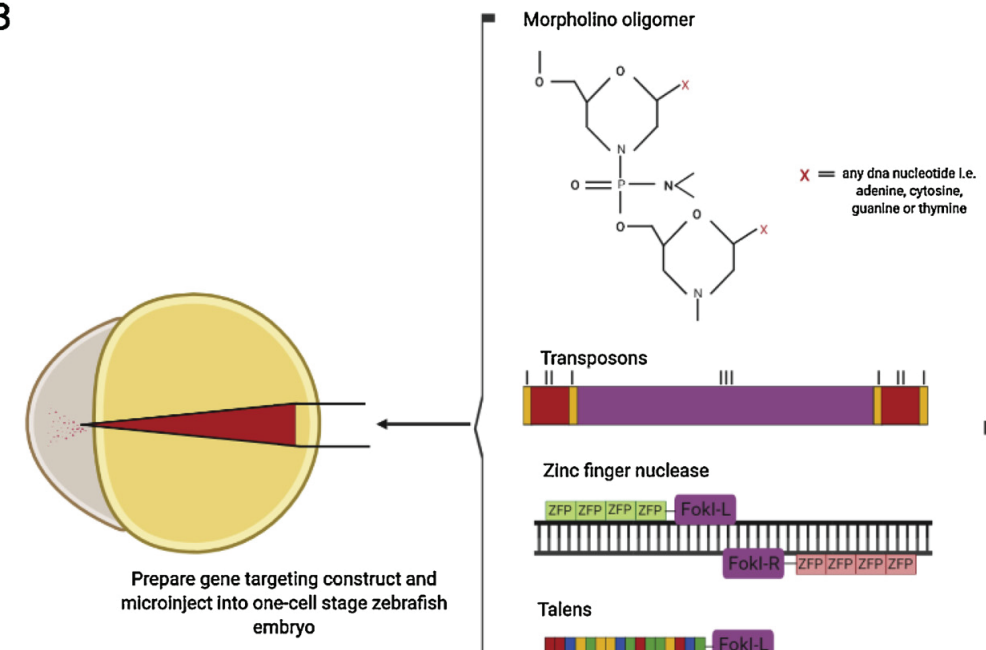

I

$=$ transposable gen

Zinc finger nuclease

ZFP ZFP ZFF ZZFP-

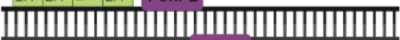

Talens

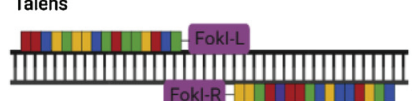

Crispr/Cas9

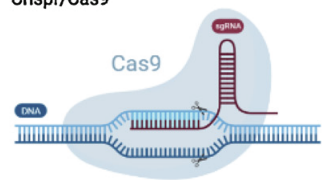

Fig. 1. Forward and reverse genetic tools available for zebrafish. A) $N$-ethyl- $N$-nitrosourea (ENU) mutagenesis is a forward genetics approach whereby point mutations are introduced into the germ cells of adult zebrafish (normally males) by bathing the fish in a solution of the highly potent chemical mutagen ENU. Mutations are introduced randomly throughout the genome and can result in either loss of function or gain of function mutations. The progeny derived from the mutagenized gametes are bred to heterozygosity or homozygosity and screened for observable phenotypes. B) Reverse genetic (gene targeting) methods. Synthetic antisense oligomers (e.g. morpholino, MO) are designed to target a gene of interest by blocking protein translation or RNA splicing. With the exception of antisense MO-mediated knock-down which is transient and non-heritable, other methods such as the use of transposon, CRISPR/Cas9, TALEN or Zinc Finger Nuclease gene editing methods lead to stable heritable mutation(s). Antisense MOs and constructs targeting the gene of interest are pressure microinjected into one-cell stage embryos.
Although confocal and two-photon microscopy enable imaging at single-cell resolution, both are still "slow" when it comes to their ability to capture very fast brain dynamics. Light-sheet microscopy therefore presents an opportunity to carry out entire brain imaging at single-cell resolution at a faster rate (Ahrens et al., 2013). Recently, light-sheet microscopy was used to explore brain network activity of grs $^{357}$ mutants, a representative genetic model of depression and demonstrating the impact of anti-depressants on this network (Burgstaller et al., 2019a, 2019b). This opens new avenues for similar studies in other neuropsychiatric diseases in the future.

\subsection{Perturbing neural circuits in zebrafish}

To understand the roles of specific circuits on behavioural outcome, it is necessary to perturb neuronal function selectively. It is also possible to perturb neural circuits in zebrafish using single-cell ablation, optogenetic stimulation or silencing (Haesemeyer and Schier, 2015). Traditional single-cell ablation studies involves the use of lasers, toxins and pharmacological agents while optogenetics requires the use of light-sensitive proteins such as channelrhodopsin or halorhodopsin (Gahtan et al., 2002; Burgess and Granato, 2007; Wyatt et al., 2015). For instance, laser ablation studies have played significant roles in our understanding of the startle response circuitry in zebrafish. This circuitry is important for the initiation of the fast C-start response in the zebrafish PPI assay (Gahtan et al., 2002; Takahashi et al., 2002, 2017;
Kohashi and Oda, 2008b). When Mauthner cells were ablated, a startle response was still observed albeit at a longer latency. These findings revealed the contribution of non-Mauthner cells in displaying the startle response and the classification of this response into slow and long latency C-start (Gahtan et al., 2002).

\subsection{Mitogen-activated protein kinase (MAP)-mapping and the Z-atlas}

MAP-mapping is an immunohistochemistry technique where fixed brain tissue is stained for phosphorylated extracellular-signaling-regulated kinase (pERK). Zebrafish are made to perform a particular behavioural task such as hunting for prey and the animals are sacrificed immediately thereafter. All active neurons during the execution of the task stain positive for pERK. One significant advantage that pERK staining has over immediate early gene markers such as $c$-Fos, is that the activation of pERK signals occurs within $5 \mathrm{~min}$ as opposed to the 15-30 minutes required for activation of c-Fos mRNA or the approximate $2 \mathrm{~h}$ translation of $\mathrm{c}$-Fos protein. The pERK method has been used to create an open source zebrafish brain atlas, Z-atlas. Once an experiment is completed, analysis of the data is entirely automated (Randlett et al., 2015). Recently, the neural activity of zebrafish larvae carrying mutations in 132 genes linked to schizophrenia from GWAS were analysed using MAP-mapping to reveal changes in brain structure and activity (Thyme et al., 2019). The MAP-mapping method, though not high resolution, allows for a high-throughput readout of brain 

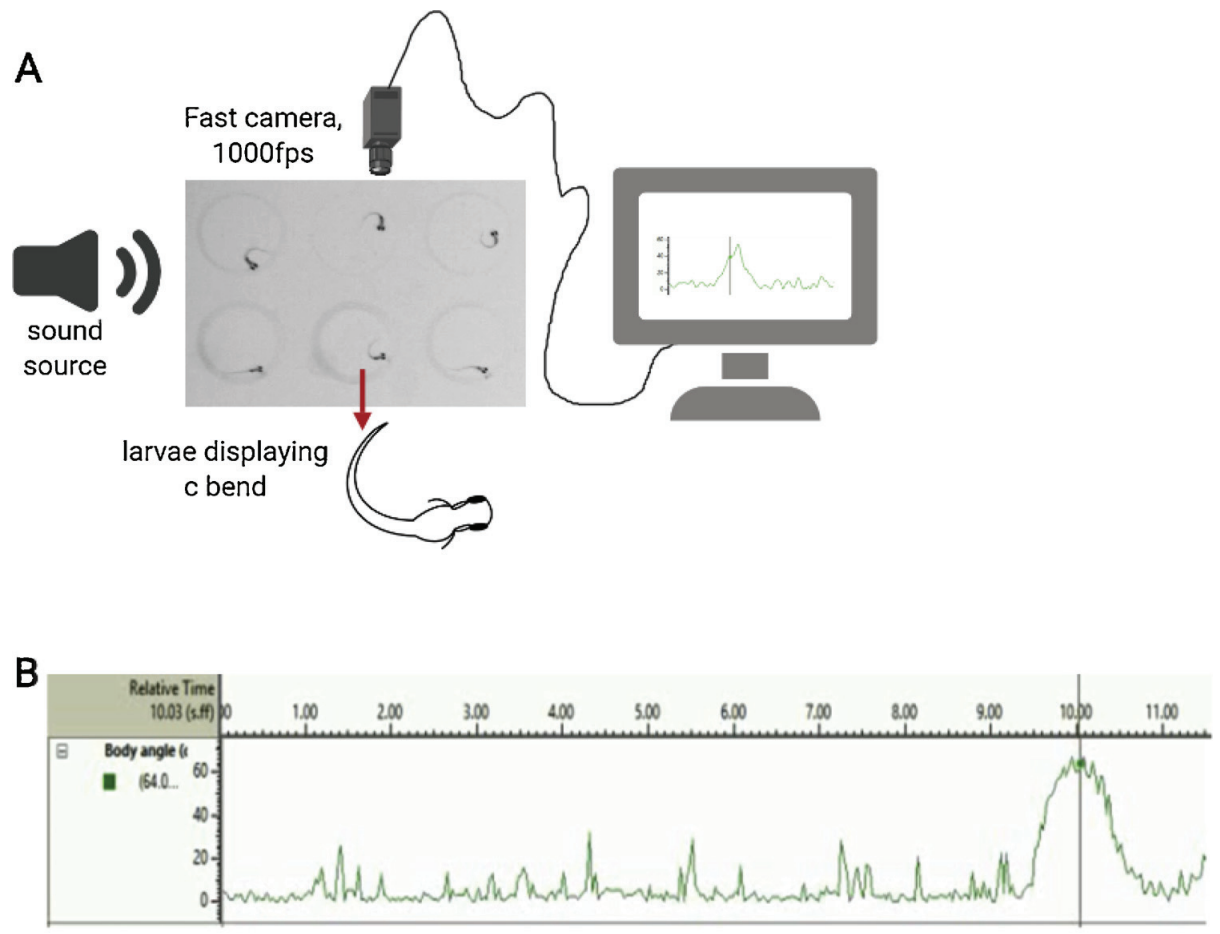

Fig. 2. Acoustic startle response in larval zebrafish. A) When a zebrafish larva is presented with an acoustic startle stimulus, it responds with an escape behaviour by displaying a Cbend motion. The C-bend occurs within milliseconds and can be captured with the use of fast cameras that record at 1000 frames per second. Insert denotes 7 days post-fertilization (dpf) wild type fish displaying the characteristic C-bend. Pre-pulse inhibition (PPI) in larval zebrafish can be studied using startle responses to sensory stimuli (e.g. acoustic stimulus) of varying intensity and frequency. B) The amplitude of the C-bend can be recorded to determine startle and non-startle sound thresholds for prepulse inhibition estimation. The bend angle of the larva in this example is 64 degrees. C) When a startle stimulus is preceded by a stimulus of lower intensity called the prepulse stimulus, the amplitude of the C-bend reduces. Prepulse stimuli at -17 and $-14 \mathrm{~dB}$ lower than the startle stimulus $(100 \mathrm{~ms} 70 \mathrm{~dB}$ pulse at $660 \mathrm{~Hz}$ ) cause a significant reduction in startle response of wild type larvae. $\mathrm{n}=80$ larvae, $* * \mathrm{p}=0.0017$ and $* * * \mathrm{p}<0.0001$, one-way ANOVA. [The PPI ZebraBox (Viewpoint, France) was used to perform the acoustic startle test while the DanioVision software (Noldus, Netherlands) was used for the offline analysis of the videos].

C

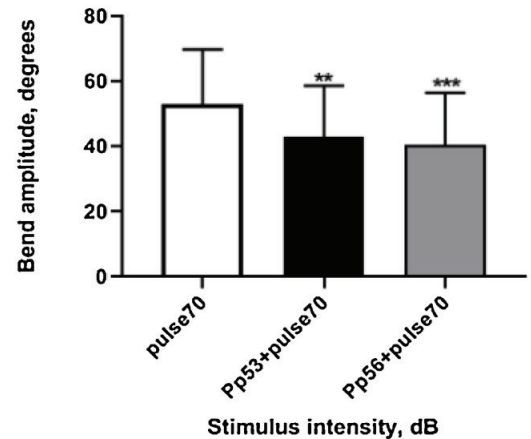

activity and gives researchers an idea of brain areas to pursue when investigating interesting gene candidates for further study.

\subsection{The relevance of zebrafish larvae in schizophrenia studies}

Although schizophrenia is a late adolescence- to early adulthood onset disease (Harrop and Trower, 2001), larval zebrafish usually between 3-10 dpf of age have been used to study schizophrenia (Giacomotto et al., 2016; Wood et al., 2009) raising concerns about the appropriateness of using larval zebrafish. Interestingly, subtle symptoms of schizophrenia are reportedly present during childhood (Fish et al., 1992; Mäki et al., 2005) suggesting that even before the advent of psychosis, some disturbances must have already occurred. Larval fish are therefore useful in gaining information at the premorbid stage of the disease. In addition, the use of larvae in schizophrenia drug screens, for instance, can serve as a filter by eliminating "uninteresting" drug candidates. The limitation with this is that larvae $<10 \mathrm{dpf}$ do not have a fully functional $\mathrm{BBB}$ and may not represent the true fate of drugs in the under a functional BBB state meaning we could get drugs that fail later in rodents or during clinical trials. It is possible to eliminate these concerns by monitoring changes at essential time points of life i.e. from the larval to juvenile and then adult stages. This will help us observe how the susceptible schizophrenia brain progresses to a mature schizophrenic brain as well as observe any potential differences in susceptible larvae that never develop full schizophrenia symptoms by the time they reach adulthood since, in humans, not all persons at high risk of schizophrenia develop the disease even if they displayed subtle abnormalities during childhood (Fish et al., 1992).

In the current review, we critically discuss pharmacological zebrafish models of schizophrenia, due to their potential usefulness for highthroughput compound screening. Moreover, we highlight insights into the basic mechanisms of action gained from these studies as well as limitations associated with these studies. Furthermore, we discuss genetic models of schizophrenia in zebrafish and highlight their usefulness in investigating the molecular consequences resulting from mutations in a specific gene of interest. We believe that this review will help the experimenter choose the most suitable zebrafish model for schizophrenia that best fits the aim of their study, while keeping in mind all the current limitations of existing models.

\section{Pharmacological models of schizophrenia in zebrafish}

The heterogeneity of schizophrenia presents significant problems in building a coherent animal model (Marcotte et al., 2001). However, 


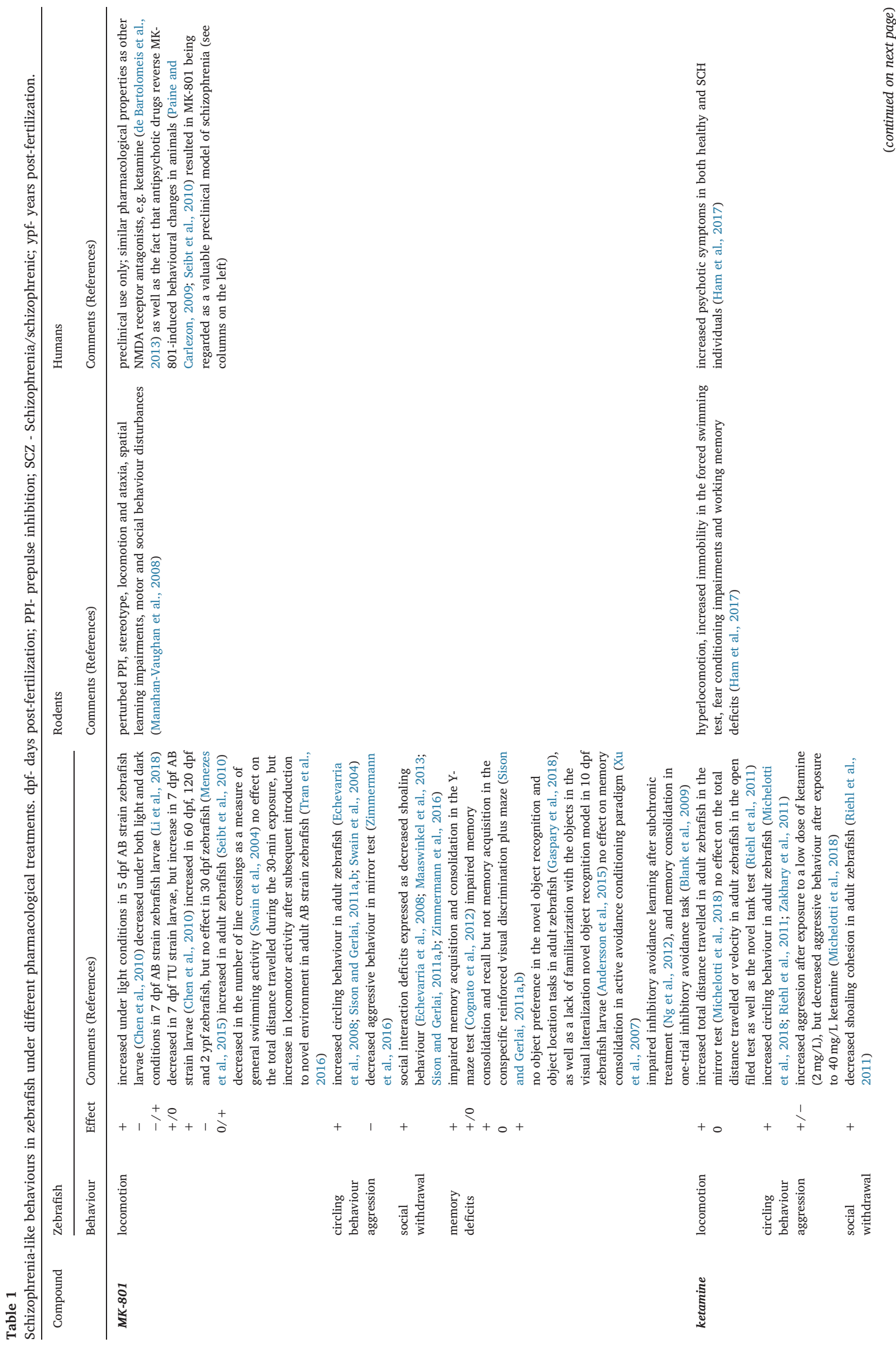




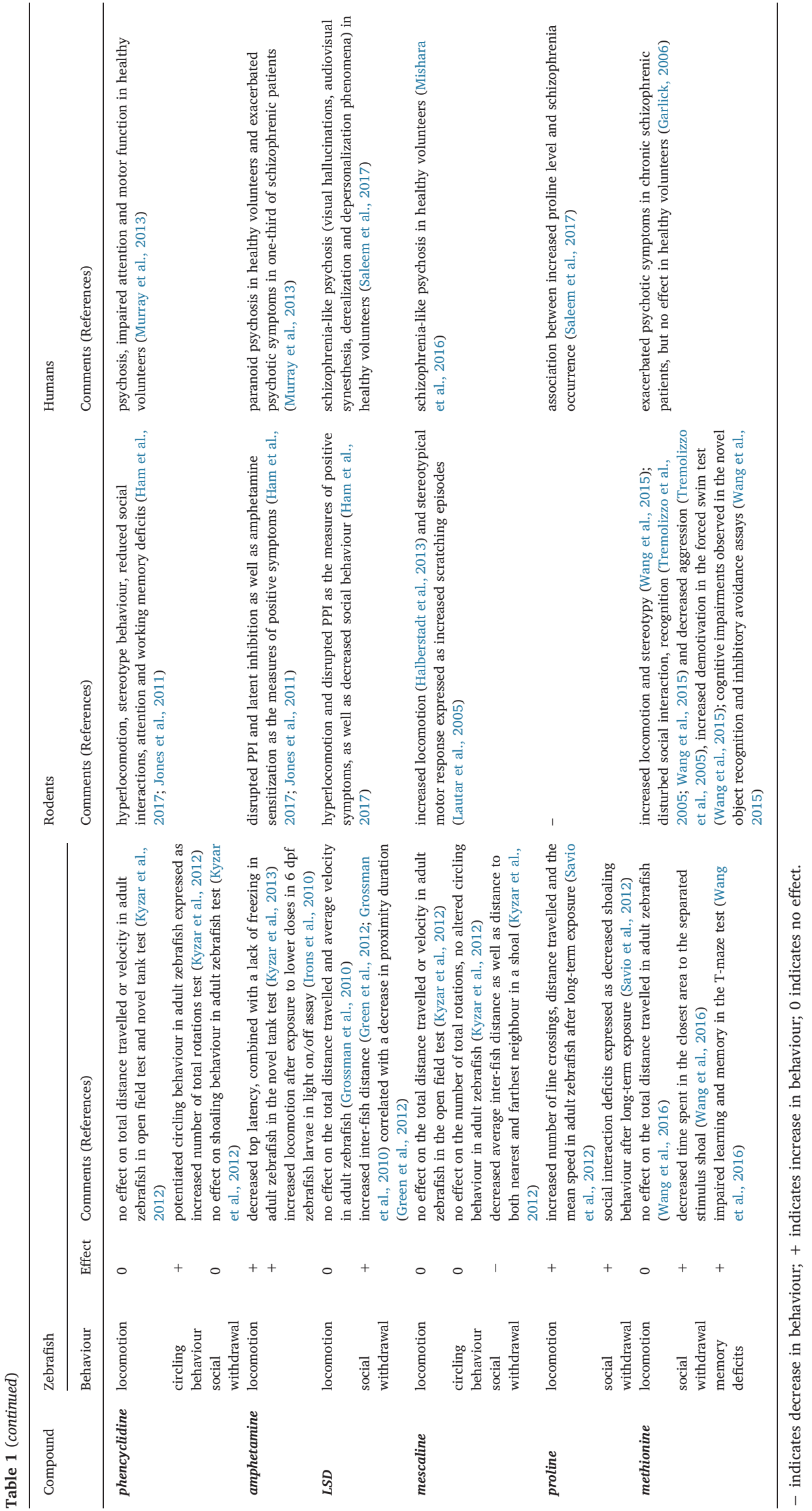




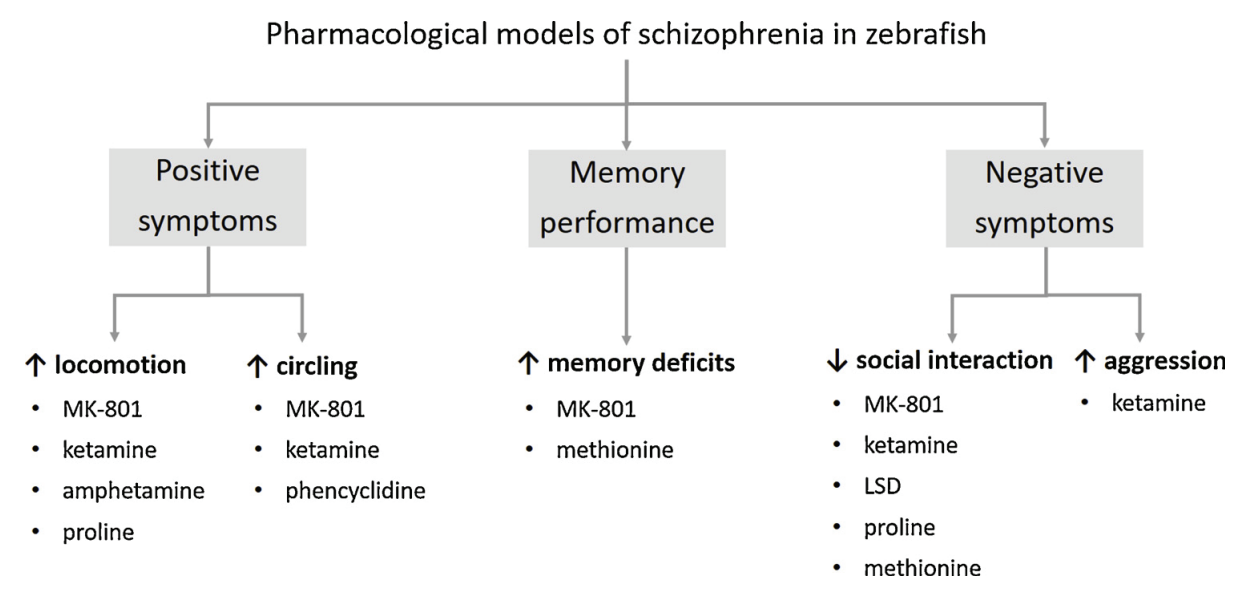

Fig. 3. Pharmacological models of schizophrenia in zebrafish. The scheme presents different symptoms of schizophrenia and its manifestations. The presented drugs have been shown to be effective in mimicking specific schizophrenia-like behaviours in zebrafish. $\uparrow$ - increased response; $\downarrow$ - decreased response.

because both psychiatric conditions result from alterations in various neurotransmitter systems, and neuronal disruptions correlate with human as well as animal behaviours (Marcotte et al., 2001), specific behavioural changes in animals represent some of the clinical symptoms of schizophrenia (Bubeníková-Valešová et al., 2008).

The purpose of the following section is to review pharmacological manipulations that lead to schizophrenia-like behaviours in zebrafish and serve as an alternative guide to other animal models of psychotic disorders. The summary of schizophrenia-like behavioural manifestations in zebrafish under different pharmacological treatments has been plotted in Table 1 and Fig. 3.

\subsection{NMDA receptor antagonists}

One of the most commonly used pharmacological models of schizophrenia has its origin from observations that NMDA receptor inhibition leads to the manifestation of schizophrenia symptoms. Blockade of the NMDA receptor with MK-801, ketamine or phencyclidine elicits a wide spectrum of schizophrenia-like behavioural changes in animals, including positive, negative and cognitive symptoms (Bubeníková-Valešová et al., 2008; see below for more detailed description). Consequently, NMDA hypofunction serves as the most complete equivalent of the human disorder, and therefore NMDA receptor antagonists will be discussed first.

\subsection{Positive symptoms}

MK-801, a noncompetitive NMDA receptor antagonist provides the most comprehensive literature on modelling schizophrenia in zebrafish. It is generally considered that hyperlocomotion in animals reflects psychotic symptoms of schizophrenia in humans (Bubeníková-Valešová et al., 2008). Although the vast majority of evidence demonstrates that MK-801 affects locomotion in both larval and adult zebrafish, the direction of changes in locomotor activity varies in different reports. An attempt to explain these inconsistencies was made by Tran et al. (2016) in their short communication, which is recommended to those seeking a more detailed discussion. Concisely, some studies revealed that MK-801 induces hyperlocomotion in larval (Chen et al., 2010; Liu et al., 2014), juvenile (Menezes et al., 2015) and adult zebrafish (Menezes et al., 2015; Seibt et al., 2010), whereas other reports found that MK-801 has either no effect on locomotor activity in zebrafish larvae (Menezes et al., 2015) or causes a decrease in locomotion in larval (Li et al., 2018; Liu et al., 2014) as well as adult zebrafish (Swain et al., 2004). The reason for inconsistent results may be due to strain differences and associated variance in neurotransmitter levels between $\mathrm{AB}$ and $\mathrm{TU}$ strains. TU strain zebrafish treated with MK-801, but not AB strain zebrafish, displayed higher expression levels of catecholamines and their metabolites, which was attributed to strain-dependent differences in behavioural responses to MK-801 (Liu et al., 2014). Similar lower levels of neurotransmitters in TU strain zebrafish have been found by Mahabir et al. (2013). In this study however, biochemical differences between untreated $\mathrm{AB}$ and $\mathrm{TU}$ strain zebrafish were correlated with a stronger tendency to develop shoaling behaviours in TU strain zebrafish (Mahabir et al., 2013). Certainly, there are strain-dependent differences in the development of the nervous system that may result in different behavioural manifestations. Little is known about genetic factors determining these differences at present. Moreover, it was also suggested that MK-801 increases locomotor activity in zebrafish in a context-dependent manner (Tran et al., 2016). For instance, although MK-801 had no effect on the total distance travelled during the 30-min exposure, a significant increase in locomotor activity in adult zebrafish was observed after subsequent introduction to a novel tank, suggesting stressdependent effects of MK-801 on locomotion in zebrafish (Tran et al., 2016).

Regarding other noncompetitive NMDA receptor antagonists, both ketamine and phencyclidine did not change locomotor activity expressed as total distance travelled or velocity in the open field test and the novel tank test (Kyzar et al., 2012; Riehl et al., 2011). However, ketamine was found to increase total distance travelled in the mirror test, which is used to study aggression-related behaviours in adult zebrafish exposed to their own reflection (Michelotti et al., 2018). Hence, it is possible that analogous to MK-801, ketamine-induced hyperlocomotion occurs in a context-dependent and stress-related manner. Moreover, it is worth noting that all above-mentioned NMDA receptor antagonists triggered circling behaviour, expressed as an increased number of rotations (Echevarria et al., 2008; Kyzar et al., 2012; Michelotti et al., 2018; Riehl et al., 2011; Sison and Gerlai, 2011b; Swain et al., 2004; Zakhary et al., 2011). It has been suggested that this change in swimming pattern reflects psychosis-like manifestations in zebrafish or may be due to hallucinogenic-like effects of NMDA receptor antagonists (Kyzar et al., 2012; Michelotti et al., 2018).

In summary, zebrafish may serve as a model organism in studies on schizophrenia-like behavioural changes reflecting positive symptoms in humans. Nevertheless, AB strain zebrafish seem to be a better choice than TU strain zebrafish as a pharmacological model of schizophrenia induced by MK-801. Properly designed experimental protocols and procedures are also of no less importance, considering the context-dependent effect of MK-801 (and presumably also ketamine) on zebrafish locomotor activity. Furthermore, circling behaviour compared to hyperlocomotion may be a better parameter for assessing positive symptoms of schizophrenia in zebrafish treated with NMDA receptor antagonists. 


\subsection{Negative symptoms}

Animal schizophrenia-like behaviours that reflect negative symptoms of the human disorder include social interaction deficits and increased aggression (Riehl et al., 2011). Aggression is an adaptive, social behaviour that animals use to establish dominance hierarchies, compete for mates or food, and defend offspring as well as territory (Jones and Norton, 2015). In the zebrafish, aggressive behaviour can be induced by placing two fish in a tank or exposing an individual to its own image (so-called mirror-induced aggression) (Jones and Norton, 2015; Guo et al., 2012). Five $\mu \mathrm{M}$ MK-801 decreased aggressive behaviour in the mirror test (Zimmermann et al., 2016) and ketamine modulated aggression in zebrafish in a dose-dependent manner. A study by Michelotti and colleagues revealed that a low dose of ketamine $(2 \mathrm{mg} / \mathrm{L})$ increased aggression-related behaviours (the number and duration of aggressive episodes), while a higher dose of ketamine $(40 \mathrm{mg} / \mathrm{L})$ decreased these parameters, as well as increased the latency to attacking their own reflection (Michelotti et al., 2018). Interestingly, the dosedependent effect of ketamine on aggressive behaviour was inversely proportional to its effect on circling behaviour (Michelotti et al., 2018). Although Zimmermann et al. (2016) did not investigate the effect of MK-801 on circling behaviour, Swain et al. (2004) demonstrated that $2 \mu \mathrm{M}$ MK-801, a lower concentration than used by Zimmermann et al. (2016), increased the number of circling events. Therefore, it can be assumed that both MK-801 and ketamine reduce aggression in zebrafish as a result of triggered stereotypic behaviours.

A good measure of social interaction in zebrafish is shoaling. Shoaling, defined as the natural assembling of fish into loose groups called shoals, is a form of social behaviour, which in natural habitats serves the role of a defence mechanism against predation, and improves foraging (Miller and Gerlai, 2007). Under laboratory conditions, shoaling behaviour can be quantified as the preference of separated individual fish towards a group of conspecifics or by assessing shoal cohesion (Guo et al., 2012). It has been demonstrated that MK-801 reduces shoaling behaviour in adult zebrafish in a wide range of concentrations from $1 \mu \mathrm{M}$ to $100 \mu \mathrm{M}$ (Echevarria et al., 2008; Maaswinkel et al., 2013; Sison and Gerlai, 2011b; Zimmermann et al., 2016). This NMDA receptor antagonist was found to induce social interaction deficits by increasing the inter-individual distance, nearest neighbour distance and farthest neighbour distance (Maaswinkel et al., 2013), as well as decreasing the time spent in the closest area to the separated stimulus shoal (Sison and Gerlai, 2011b). Interestingly, Maaswinkel et al. (2013) reported that MK-801 can decrease social cohesion for the entire shoal also when only one of four fish is pretreated with the drug, which suggests that zebrafish may change their behaviours in response to the drug-altered behaviour of their conspecific. Of the other two discussed NMDA receptor antagonists, only ketamine, but not phencyclidine, was shown to cause social withdrawal in zebrafish, increasing the average inter-individual distance (Kyzar et al., 2012; Riehl et al., 2011 respectively).

Overall, social interaction deficits expressed as decreased shoaling provide a valuable tool for modelling negative schizophrenia symptoms in zebrafish exposed to NMDA receptor antagonists. Although the assessment of aggressive behaviour may also provide interesting insights into schizophrenia-like behaviours in zebrafish, it should be correlated with other behavioural changes induced by NMDA receptor antagonists.

\subsection{Memory deficits}

Impairment of cognitive functions is another behavioural manifestation related to the symptoms of schizophrenia (Bubeníková-Valešová et al., 2008). Although reports on the effect of NMDA receptor antagonists on memory performance in zebrafish are not as comprehensive as in the case of other animal models, they provide an interesting insight into modelling memory deficits with MK-801. On the whole, acute MK-801 impaired memory acquisition and memory consolidation in the Y-maze test (Cognato et al., 2012), in the plus maze test (including memory recall) (Sison and Gerlai, 2011a) and one-trial inhibitory avoidance task (Blank et al., 2009). Moreover, MK-801 disturbed the familiarization phase in the novel object recognition test in adult zebrafish (Gaspary et al., 2018) and larvae (Andersson et al., 2015), suggesting impairment of attention and working memory. Finally, adult zebrafish subchronically treated with MK-801 (1-h immersion for four consecutive days) maintained the tendency to swim in the deep environment, naturally preferable but correlated with an electric shock, which indicates impairment of inhibitory avoidance learning ( $\mathrm{Ng}$ et al., 2012).

On the other hand, there are reports that did not reveal memory deficits in zebrafish treated with MK-801, in terms of memory acquisition (Sison and Gerlai, 2011a) and memory consolidation (Xu et al., 2007). However, the vast majority of evidence (see above) confirms that zebrafish treated with MK-801 may serve as a pharmacological model of memory deficits mimicking cognitive symptoms of schizophrenia. Taking into consideration that MK-801 evokes behavioural changes reflecting all three dimensions of the human disorder (positive, negative and cognitive), it can easily be concluded that this NMDA receptor antagonist builds the most coherent pharmacological model of schizophrenia in zebrafish.

\subsection{Amphetamine and hallucinogenic drugs}

The dopamine hypothesis of schizophrenia postulates that a hyperdopaminergic state in the mesolimbic pathway is linked with positive symptoms of schizophrenia (Marcotte et al., 2001). Therefore, attempts have been made to use dopamine agonists, such as amphetamine, in modelling positive schizophrenia-like behaviours in animals. In fact, amphetamine provokes hyperlocomotion and stereotypic behaviours in rodents, which can be correlated with positive psychotic symptoms of schizophrenia (Marcotte et al., 2001). Considering adult zebrafish, Kyzar et al. (2013) demonstrated that acute amphetamine decreased both the top latency and time spent frozen in the novel tank test, indicating an increase in locomotor activity. Moreover, amphetamine affected locomotion also in zebrafish larvae in the light on/off assay, showing an inverted U-shaped dose-effect curve with a maximum hyperlocomotion response at the concentration of $0.7 \mu \mathrm{M}$ (Irons et al., 2014). Unfortunately, both studies did not investigate stereotypic behaviours in zebrafish (e.g. circling behaviour), which could have been an interesting complement to the evaluation of amphetamine-induced changes in locomotion.

Because the serotoninergic system has also been implicated in schizophrenia (Marcotte et al., 2001; Selvaraj et al., 2014), drugs mediating their effects through serotonin receptors, such as hallucinogenics (e.g. LSD and mescaline), can be used as pharmacological models of schizophrenia in animals. There are studies that investigated LSDand mescaline-induced behavioural changes in zebrafish (Green et al., 2012; Grossman et al., 2010; Kyzar et al., 2012). None of them, however, have provided convincing evidence that hallucinogenic drugs may serve as reliable pharmacological models of positive symptoms of schizophrenia. Specifically, mescaline did not affect circling behaviour in zebrafish (Kyzar et al., 2012), and no locomotor changes were observed after exposing adult fish to either mescaline or LSD (Grossman et al., 2010; Kyzar et al., 2012). Moreover, not only did mescaline not elicit social withdrawal in zebrafish but rather, it increased social cohesion in a shoal (Kyzar et al., 2012), thereby, failing to produce negative schizophrenia-like symptoms as well. However, LSD-treated zebrafish displayed social interaction deficits expressed as increased inter-fish distance (Green et al., 2012; Grossman et al., 2010) and decreased proximity duration, defined as the average amount of time a fish spent close to another individual (Green et al., 2012).

In conclusion, amphetamine produces psychotic-like changes in zebrafish behaviour and can therefore be used in modelling positive 
symptoms of schizophrenia. Hallucinogenic drugs are much less useful in triggering schizophrenia-like symptoms in zebrafish with an advantage in favour of LSD over mescaline in respect of inducing social interaction deficits.

\subsection{Amino acids}

It has been reported that increased levels of many amino acids, i.e. glutamate, glycine and D-serine, can be found in blood samples of schizophrenic patients (Saleem et al., 2017). Thus, along with NMDA hypofunction and disturbed dopaminergic neurotransmission, amino acids can also serve as targets in designing animal pharmacological models of schizophrenia. Indeed, methionine, an essential amino acid in humans, has been shown to elicit schizophrenia-like behaviours in mice, demonstrated as disturbed social interactions and social recognition (Tremolizzo et al., 2005). Similarly, methionine-induced social withdrawal was found in adult zebrafish, which exhibited a reduced preference for a stimulus group of conspecifics in the shoaling paradigm. Moreover, methionine-treated zebrafish failed to remember the location of the reservoir in the T-maze test but did not express any locomotion changes in the novel tank test (Wang et al., 2016). Locomotor activity in zebrafish has also been disrupted by another amino acid. Savio et al. (2012) investigated the influence of proline, a proteinogenic amino acid, on the number of line crossings, distance travelled and mean speed. Proline increased all three tested parameters, but only after subchronic 7-day exposure, which also led to social interaction deficits in zebrafish exposed to a stimulus shoal in the shoaling paradigm (Savio et al., 2012). To conclude, both methionine and proline can be used as pharmacological models of social interaction deficits in zebrafish. Additionally, proline-induced hyperlocomotion reflects positive psychotic-like symptoms in zebrafish, while methionine-caused learning impairment mimics memory deficits observed in the course of schizophrenia.

\section{Insight into genetic aspects of schizophrenia using zebrafish}

One of the reasons for limited progress in elucidating the genetic background of schizophrenia until recently was the lack of proper, informative and easy animal models to mimic aspects of schizophrenia symptoms in humans. It seems that zebrafish may be a valuable tool to fill this gap and provide insight into genetic factors determining the occurrence of schizophrenia in humans. Notably, zebrafish orthologs have been identified for $88 \%$ of all currently known schizophrenia risk genes (Kasap et al., 2018), which is even higher than the overall homology between the two genomes (i.e. 70\%) (Howe et al., 2013). Although GWAS have identified over 100 risk loci for schizophrenia, there is a lack of conclusion as to how they confer liability to the disease. Thus, zebrafish genetic models of schizophrenia open new prospects for investigation of schizophrenia risk genes and additionally, their interaction with other genes as well as environmental factors (i.e. genetic modifiers and epigenetic mechanisms).

\subsection{DISC1 and NRG1}

One of the best-characterized schizophrenia risk genes is DISC1 (disrupted-in-schizophrenia), which was first reported in a large Scottish family, where its members suffered from schizophrenia and affective disorders (Wood et al., 2009). Using zebrafish models, the molecular background of schizophrenia associated with loss-of-function in the disc1 gene has been, at least partially determined (for a summary and comparison with rodent and human studies, see Table 2). Antisense-MO-mediated knock-down studies revealed a critical role for disc1 in oligodendrocyte and cerebellar neuron development by uncovering its function in the specification of oligo2-positive precursor cells in the zebrafish hindbrain. Moreover, knock-down of nrg1 (neuregulin 1), which is involved in the myelination process, induced similar phenotypic changes in larval zebrafish through the same mechanism as observed in disc-1 morphants (Wood et al., 2009). Finally, the study concluded that both genes are highly important for the development of different neural cell types originating from oligo2-positive precursor cells (Wood et al., 2009). In a follow-up study, the same group revealed that sonic hedgehog (Shh) signalling was disrupted in smoothened (i.e. disc1) knock-down mutants (Boyd et al., 2015). Since Shh is considered to be essential for oligo2-positive precursor cells, the absence of these cells in morphants was associated with the complete loss of Shh signalling. Also, Drerup et al. (2009) revealed that disc1 is expressed in premigratory neural crest cells and its loss-of-function causes their aberrant migration as a result of enhanced expression of foxd3 and sox10 transcription factors. Moreover, two other groups in parallel revealed that disc1 loss-of-function reduces GSK3 $\beta$ activity, leading to inhibition of $\beta$-catenin-mediated Wnt signalling (De Rienzo et al., 2011; Singh et al., 2011). Importantly, zebrafish larvae displayed abnormal movement of dorsolateral cells during gastrulation and, as a consequence, the formation of a U-shaped body with truncated tail (Singh et al., 2011). This was mediated by the non-canonical Wnt pathway (i.e. Daam and Rho signalling), as evidenced by the fact that the phenotype was rescued by GSK3ß-overproduction (De Rienzo et al., 2011). Eventually, Eachus et al. (2017) investigated, using both larval and adult disc1 mutant zebrafish, the mechanism through which disc1 affects the stress response and function of the hypothalamic-pituitary-interrenal (HPI) axis in these animals. In a series of experiments, the HPI axis (the equivalent of the human hypothalamic-pituitary-adrenal axis) in zebrafish was shown to be evolutionary conserved. Using two different mutant lines (each carrying a nonsense mutation for disc1) it was observed that adult fish exhibited anxiety-like behaviour as well as an aberrant stress response to an acute stressor. For example, in an open field test adult mutants exhibited increased baseline freezing compared to wild type counterparts. Similarly, when exposed to an acute stressor, mutants did not increase bottom dwelling (in the tank diving test) compared to wild type siblings, which indicated an aberrant response to stress in these fish. In addition, mutant larvae did not modulate shoal cohesion under stress. Subsequently, the authors aimed to answer how the mutations in this gene affect progenitor cells and differentiation of neurons in the hypothalamus of zebrafish larvae. Briefly, they reported that in mutant larvae 1) the expression of transcription factor rh3 (retinal homeobox 3), which demarcates progenitor cells in the hypothalamus to give rise to specific neuronal populations (e.g. ventromedial neurons), was reduced, 2) the expression of nuclear factor $f f 1 b$ in the ventromedial nucleus of the hypothalamus was enhanced, while in steroidogenic cells, expression was normal, 3) hypothalamic pomc + cells (proopiomelanocortin, which defines Arc-like neurons in hypothalamus) were disorganized, and finally, 4) the number of hypothalamic crh + (corticotropin-releasing hormone) neurons was reduced. Eventually, the authors concluded that disc1 is crucial for the maintenance of the appropriate number of hypothalamic progenitor cells, proper differentiation of neurons in this structure and normal function of the HPI axis in larval and adult zebrafish. On the basis of all above-mentioned papers, we may conclude that the zebrafish might be a highly valuable tool not only in the evaluation of molecular pathways underlying schizophrenia but also in genetic-environment interactions.

\section{2. $\mathrm{TH}$}

It is widely believed that dopamine signalling is aberrant in schizophrenic patients. Thus, most of the currently available drugs influence the imbalance between the serotoninergic and dopaminergic systems. Although the onset of schizophrenia occurs typically in adolescence or early-adulthood, different environmental factors (e.g. maternal viral infections or chronic stress during pregnancy) induce changes in dopamine signalling in the critical period during neurodevelopment which, in turn, has implications later in life. Formella et al. (2012) investigated, using a series of behavioural experiments (i.e. 


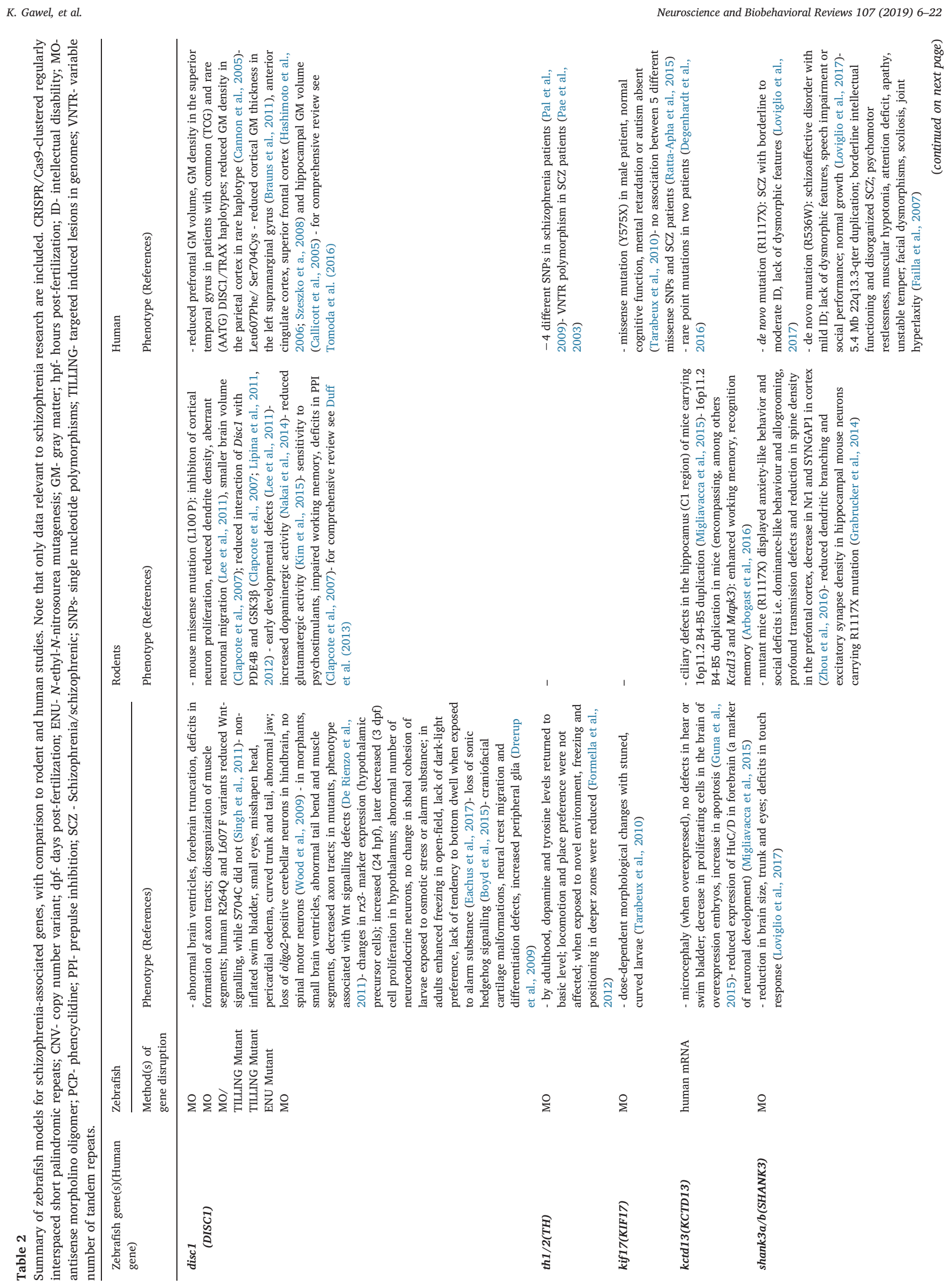




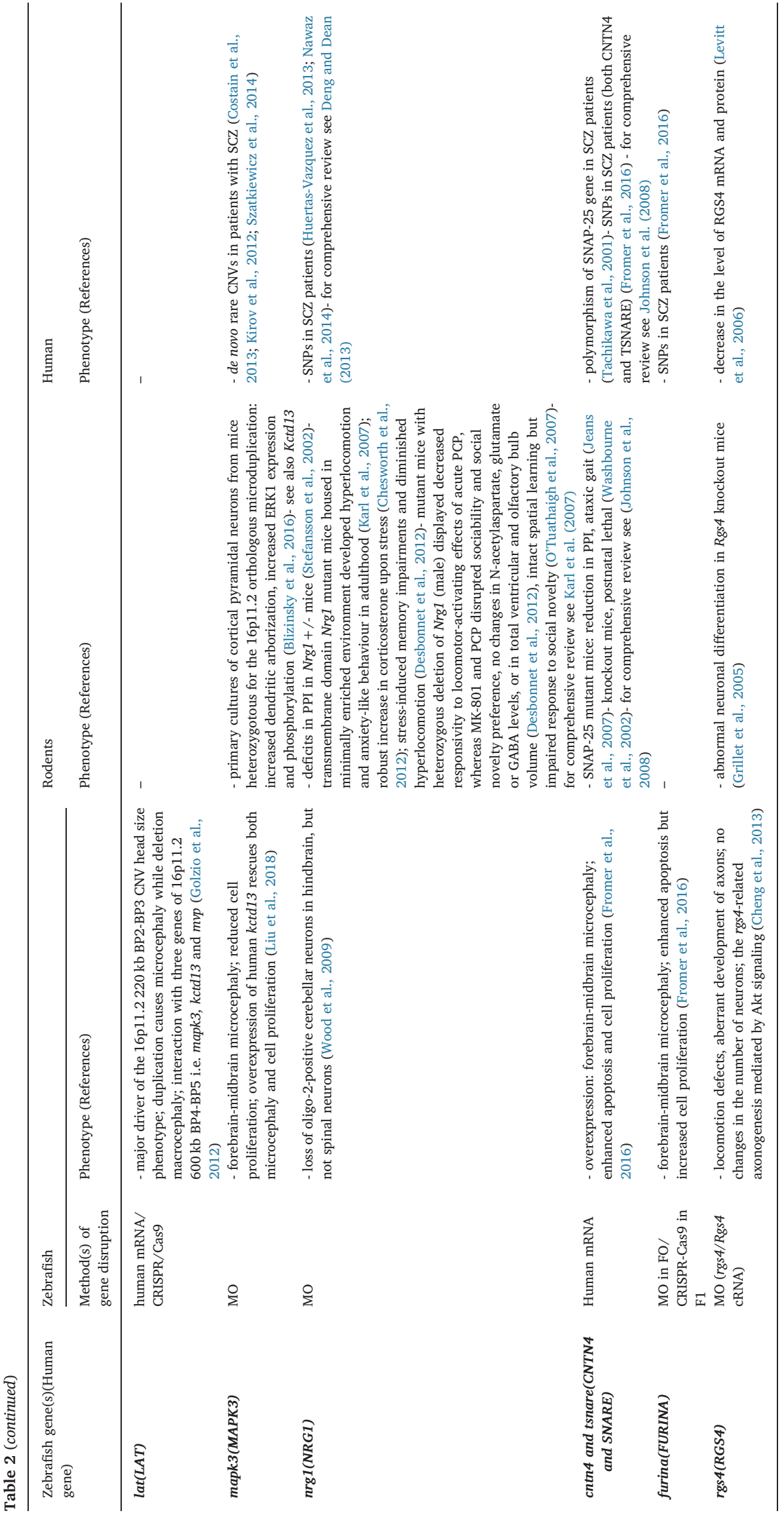


open field, place preference and novel diving tank) in adult fish, if/how disruption of dopamine signalling in early-life stages affects the adult behavioural phenotype. More specifically, they induced transient knock-down of tyrosine hydroxylase using MOs targeting th1 zebrafish gene (Formella et al., 2012). The enzyme encoded by this gene is the rate-limiting enzyme which catalyses hydroxylation from L-tyrosine to L-DOPA, the precursor of dopamine. Although transient th1 loss-offunction perturbed dopaminergic signalling in larval zebrafish, the dopamine level in adult MO-injected zebrafish brains did not differ compared to control littermates. In addition, basic behaviour such as locomotion (dopamine-dependent) did not change. Nevertheless, the authors observed that adult zebrafish exhibited a lower level of freezing and immediate positioning in deeper zones of a novel environment, which indicates a potential anxiolytic-like effect induced by early-life loss-of-function in this gene. The molecular mechanism of this perturbation has not been investigated. However, authors clearly stated that due to the fact that this gene in zebrafish is duplicated, the other allele, i.e. th2, even though less expressed in the zebrafish brain, could potentially compensate for the observed phenotype (Formella et al., 2012).

\subsection{KIF17}

In a very elegant study, Tarabeux et al. (2010) studied a de novo nonsense mutation in KIF17, a gene encoding Kinesin 17, a synaptic motor protein, in one patient with schizophrenia. To confirm the hypothesis that the mutation (Y575X) might have a pathogenic effect, they knocked down the expression of kif17 in zebrafish using two different types of MOs. The first MO was designed to target the same affected exon as in the patient (i.e. exon 9) while the second MO blocked the AUG codon. The idea behind this approach was to 1) mimic the aspects of the human mutation and confirm or exclude whether this mutation might cause the synthesis of a truncated protein, and 2) determine whether protein loss-of-function would cause the same, weaker, or more severe phenotype in zebrafish compared to that caused by the truncated protein. In both cases, the authors observed that the injection of MO resulted in dose-dependent morphological defects, including curved body axis. No differences in phenotypic outcome were observed between the two MOs, leading to the conclusion that the truncated protein is presumably non-functional and might, therefore, be pathogenic in this patient. Although they did not test any drugs in their model, this approach opens new perspectives for the future utility of zebrafish in precision medicine, especially for pharmacoresistant patients who do not respond to currently available treatment options.

\subsection{SHANK3}

The study described by Gauthier et al. (2010) deserves special attention. As part of the "Disease to Synapse" project, the authors investigated, using human subjects suffering from schizophrenia, the occurrence of mutations in the SHANK3 gene previously linked with autism (Kozol et al., 2015; Liu et al., 2018), and identified two different de novo mutations (R1117X, nonsense and R539W, missense). Surprisingly, the patients carrying these mutations did not exhibit autism (or autism-like) symptoms, but rather, schizophrenia with onset during early adulthood. To determine the pathogenicity of these variants in human patients, they knocked down two orthologs of this gene in zebrafish. Antisense MO-mediated knock-down in zebrafish embryos resulted in a reduction in the size of the head and trunk as well as deficits in the touch evoked response. Injection of wild type rat Shank3 mRNA, partially rescued the phenotypic changes. On the other hand, injection of rat Shank3 mRNA bearing the equivalent human R1117X, but not R539W, failed to rescue the phenotype.

\section{5. $R G S 4$}

RGS4, a modulator of G-protein coupled signalling, specifically regulates $\mathrm{G}$ proteins of the Gai/o and Gaq families (Bansal et al., 2007). Cheng et al. (2013) aimed for the first time, to investigate the role of rgs 4 in the developing nervous system using the zebrafish as a model. The experiment was preceded by characterization of rgs 4 in zebrafish in comparison to other species (chicken, Xenopus laevis, mice, rat). With regard to humans, for which 5 splice variants of RGS4 were identified, it was revealed that isoforms 1 and 2 of zebrafish rgs 4 possessed the highest degree of similarity to the human protein (47\% identical and $69 \%$ conserved amino acids). Interestingly, syntenic comparison with mammalian homologues and analysis of the exon-intron structure of vertebrate Rgs4 revealed that zebrafish and mammalian genes are strictly conserved, which suggests a common ancestral gene for Rgs 4 throughout the evolution. MO knock-down of rgs 4 did not cause severe morphological defects, but locomotor disturbances (spontaneous coiling, touch response and swimming behaviour) occurred. The analysis of the neuronal precursor marker neurogenin1, inter-neuron marker pax $2 a$, post-mitotic marker $H u C / D$, primary motor neuron marker islet $1 / 2$ and cell apoptosis marker caspase-3, did not reveal any alteration in the number or localization of different types of neurons. However, it was revealed that motoneuron projections and Rohon-Beard sensory axons in the spinal cord were truncated in rgs 4 morphants compared to control larvae, but muscle morphology was not affected. Moreover, knock-down of rgs 4 caused defects in Mauthner cell axon pathfinding in the hindbrain of morphants as well as trigeminal axons, which all together are essential for the zebrafish touch response. To test whether pharmacological inhibition of rgs4 protein may induce the same/similar phenotype, authors injected CCG-4986 (a selective inhibitor of the RGS4 protein) into the zebrafish embryos. Indeed, they observed a similar phenotype for CCG-4986-treated embryos as for rgs 4 morphants (i.e. motility disturbances, abnormal axonal defects in hindbrain, spinal cord and trigeminal nerves). To provide further insight into mechanisms of rgs4-related pathogenicity, authors investigated the changes at the molecular level and indicated that rgs4dependent regulation of axonogenesis in zebrafish is mediated by PI3KAkt (major signalling pathway downstream of receptor tyrosine kinases). In summary, this study shed light on the molecular pathways involved in rgs4-deficiency, but the relevance of these data for schizophrenic patients (Levitt et al., 2006) needs further investigation.

\subsection{Copy number variants}

As was previously mentioned, CNVs (i.e. duplication or deletion of parts of the genome) has been implicated in several neurodevelopmental disorders, like autism or schizophrenia. One of the most commonly affected regions in humans is 22q11.2, in which micro-deletion (2.5 Mb) is manifested by microcephaly or intellectual disability. Using proteomic approaches, it was revealed that this region comprises 46 genes, from which 41 are expressed in the human brain (Guna et al., 2015). For comparison, 40 (86.9\%) and 37 (80.4\%) homologues have been identified in the mouse and zebrafish respectively. This comparative analysis clearly supports the potential of the zebrafish model in investigating the genetic causes of schizophrenia.

Although the manipulation of single genes in rodents is relatively easy nowadays, multigenic mutations are still challenging. Due to the relative ease of methods to manipulate the zebrafish genome, it may be possible to overcome this obstacle. Undoubtedly, this makes zebrafish an ideal in vivo model not only to test single genes easily but also genegene interactions. Loviglio et al. (2017) assessed the drivers of the distal 16 p11.2 duplication, which in humans contributes significantly to psychiatric disorders like autism and schizophrenia. In zebrafish, this locus encompasses nine genes. By overexpressing each of the genes, they observed that only lat duplication (gene coding a protein belonging to $\mathrm{T}$ cell receptor's group; $220 \mathrm{~kb}$, BP2-BP3) induced a 
reduction in proliferating cells in the brain with concomitant microcephaly. Subsequently, they co-injected lat with each of the other eight transcripts and assessed the number of proliferating cells in zebrafish brains. No changes when co-injecting lat with other genes were observed, supporting the conclusion that lat was the single driver of the observed phenotype, but only in this part of the genome. However, when co-injected with $k c t d 13$, mapk3 or mvp mRNA, i.e. modifiers of $16 \mathrm{p} 11.2600 \mathrm{~kb}$ BP4-BP4 CNVs, the number of proliferating cells decreased substantially $(25 \%, 20 \%$ or $22 \%$, respectively) compared to $18 \%$ in larvae injected only with lat.

Gusev et al. (2018) identified mapk3 (through mRNA overexpression), a gene within the $16 \mathrm{p} 11.2$ region, as a functional trigger of microcephaly and reduction in proliferating cells in zebrafish brains. Because the overexpression of another transcript i.e. kctd13 (Golzio et al., 2012) had previously been described as associated with similar defects, they performed the study to confirm/exclude whether mapk3 may be a potent driver of $k c t d 13$ in zebrafish. For this purpose, they suppressed mapk3 in kctd13-overexpressing embryos and rescued the $k c t d 13$ phenotype. In summary, both papers underscored the utility of zebrafish in investigating epistasis.

\subsection{Other genes}

A recently published paper by Thyme et al. (2019) undoubtedly revealed the potential of zebrafish for schizophrenia research by prioritizing more than 30 schizophrenia-related genes for further indepth investigation. In this seminal paper, the authors analysed the landscape of human schizophrenia-related genes (identified by GWAS) by mutating 132 zebrafish orthologs and performed further phenotypic characterization. Using multiple guide RNAs (gRNAs) or CRISPR/Cas9 technology, the candidate genes found within or neighboring 108 human loci previously linked to schizophrenia were mutated (with predicted truncations), and all copies (if duplicated) were assessed together. In order to identify relevant candidates for further in-depth examination, the authors created a comprehensive atlas of schizophrenia-related phenotypes which combines behavioural (i.e. locomotor acivity and PPI), whole-brain imaging as well as brain structural differences data. Notably, the c.a. $78 \%$ of the genes analysed by Thyme et al. (2019) were underexplored regarding their schizophrenia-related function. The other $22 \%$ genes were already characterized in the literature, and authors referred to them throughout the study as "unambiguously associated genes".

Since the detailed description of every single gene investigated by Thyme et al. (2019) is far beyond the scope of this review, herein we will provide a summary of the findings obtained from this study as well as comment on the translational value of these data. Firstly, this study clearly reinforced the data obtained from previously investigated schizophrenia-related genes in human and rodents studies (e.g. Gria1, Cacna1c, Cacna2b or Foxg1), thus highlighting the power of zebrafish in deciphering molecular and physiological pathways underlying schizophrenia but also its utility in phenocopying human symptoms. Secondly, the authors pointed out and provided phenotypic analysis for more than 20 genes, which seem to possess high relevance to schizophrenia in humans. For some of them (e.g. znf536, slc35g2 or gramd1b) biological function has not been described so far, while for others (e.g. cnmm2, gigyf2 or znf804a) there are some literature data. However, this study also revealed novel phenotypes. Moreover, authors pointed out some genes (e.g. cnmm2, elfn1, rora, gigyf2 or astn1) as drivers in multigene loci because of their shared pathological phenotypes with "unambiguously associated genes". Disruption of these genes caused PPI deficits and forebrain dysfunction at the whole-brain level. They also provided a list of 16 , at first glance, unrelated genes which formed the larger group of shared phenotypes, affecting the zebrafish pallium or optic tectum. This comprehensive analysis also has relevance towards other neurodevelopmental diseases, since the mutations in the genes of interest may be involved in overlapping disorders, like epilepsy, depression and autism. Furthermore, the study revealed similarities in schizophrenia-related phenotypes between zebrafish and mammals, both at the anatomical (e.g. rora, akt3 or bcl11b) and behavioural levels (akt3, gpm6). In addition, this study demonstrates how commonly used laboratory techniques can be applied toward large-scale screening of phenotypes for multiple mutants in parallel, an approach that would be impossible to conducted using rodent models.

\section{Concluding remarks}

As highlighted above, the zebrafish is both an attractive and alternative animal model for the investigation of schizophrenia, especially in the context of understanding the genetic contribution to this disorder. Specifically, genetic models (e.g. disc1) are suitable for determining the sequence of molecular events culminating in the onset of schizophrenia. However, when using zebrafish, one should also keep in mind some limitations. First of all, when planning experiments one should remember that, even though the zebrafish genome is highly conserved and shares almost $70 \%$ homology to the human genome, a number of genes are duplicated, leading to possible compensatory mechanisms that could mask loss-of-function phenotypes. Moreover, taking into account the prevalent criticisms surrounding MO-mediated knock-downs (e.g. off-target effects associated with p53-mediated apoptosis), rigorous multiple controls to determine the specificity of the observed knock-down phenotype (e.g. mRNA rescue or the use of two different MOs) is absolutely required. Ideally, a comparison of the MO phenotype with that of genetic mutants is preferred. Similarly, when several RNA splice variants of a given gene are known, MOs targeting all variants should be tested separately and in combination to determine which paralog/s is/are responsible for the observed phenotype. We believe that genetic models of schizophrenia might also be useful in precision medicine, in particular, for those patients who are unresponsive to available pharmacotherapy.

Zebrafish pharmacological models of schizophrenia mimic some aspects of the human condition and share similarities with rodent models. These models could be particularly useful for high-throughput screening of novel compounds with the potential to target the dopaminergic and/or glutamatergic system. Nevertheless, when conducting these experiments, some additional aspects should be considered. One of the most critical points seems to be with regard to strain differences. Also, on the basis of available data, it appears that MK-801 induces most comprehensively all aspects of human symptoms. Moreover, circling behaviour might be a better indicator of positive symptoms than locomotor activity. On the other hand, aggressive behaviour should not necessarily be considered as a marker of negative symptoms, as it might be perturbed by circling behaviour, giving false results. In this regard, shoaling may be a better tool for estimating negative symptoms in zebrafish. Undoubtedly, zebrafish may serve as a useful pharmacological model for studies of schizophrenia. However, some discrepancies in the literature force further validation in order to prove zebrafish reliability with regard to pharmacological models of schizophrenia.

Although the use of zebrafish in schizophrenia research, compared to rodents, is still in its infancy (for details see Table 1 and 2), the number of advantages it presents makes it a very useful model to complement rodent work. Studying aspects of polygenicity (e.g. through intercrossing mutant lines) can be more rapidly carried out compared to rodents. The existence of large zebrafish mutant repositories (Zebrafish Mutation Project, Sanger Institute, UK, https:// www.sanger.ac.uk/resources/zebrafish/zmp/) is also important to note, as the availability of lines carrying defined mutations allows researchers to quite easily investigate molecular mechanisms of the disease, which, when using rodent models is still challenging. The use of zebrafish as a model for identifying genetic modifiers, its amenability to carrying out whole-brain imaging and the available plethora of transgenic reporter lines to investigate mechanisms of disease pathogenesis in the context of a developing brain are other important features. Last 
but not least, the zebrafish enables in vivo, real-time investigation and testing of mechanistic hypotheses through pharmacological intervention with relative speed and ease.

\section{Authors' contributions}

All authors wrote the first draft of the manuscript. KG and AM prepared the tables. NSB and AM prepared figures. KG and CVE prepared the final and revised version of the manuscript. All authors read and approved the final manuscript.

\section{Declaration of competing interest}

Nothing to declare.

\section{Acknowledgements}

KG was funded by a mobility grant from the Polish Ministry of Science and Higher Education within the programme "Mobilność Plus V" (decision nr 1649/1/MOB/V/17/2018/0; 01.01.2018-31.12.2018). KG has also received funding from the European Union's Horizon 2020 research and innovation programme under the Marie Skłodowska-Curie (grant agreement No. 798703-GEMZ-H2020-MSCA-IF-2017). This work was supported by the Research Council of Norway (ISP, BIOTEK2021/ DigiBrain).

\section{References}

Ahrens, M.B., Orger, M.B., Robson, D.N., Li, J.M., Keller, P.J., 2013. Whole-brain functional imaging at cellular resolution using light-sheet microscopy. Nat. Methods 10 (5), 413-420. https://doi.org/10.1038/nmeth.2434.

Andersson, M.A., Ek, F., Olsson, R., 2015. Using visual lateralization to model learning and memory in zebrafish larvae. Sci. Rep. 5, 8667. https://doi.org/10.1038/ srep08667.

Arbogast, T., Ouagazzal, A.-M., Chevalier, C., Kopanitsa, M., Afinowi, N., Migliavacca, E., Cowling, B.S., Birling, M.C., Champy, M.F., Reymond, A., Herault, Y., 2016. Reciprocal effects on neurocognitive and metabolic phenotypes in mouse models of 16p11.2 deletion and duplication syndromes. PLoS Genet. 12 (2), e1005709. https:// doi.org/10.1371/journal.pgen.1005709.

Bansal, G., Druey, K.M., Xie, Z., 2007. R4 RGS proteins: regulation of $\mathrm{g}$ protein signaling and beyond. Pharmacol. Ther. 116 (3), 473-495. https://doi.org/10.1016/j. pharmthera.2007.09.005.

Bhandiwad, A.A., Zeddies, D.G., Raible, D.W., Rubel, E.W., Sisneros, J.A., 2013. Auditory sensitivity of larval zebrafish (Danio rerio) measured using a behavioral prepulse inhibition assay. J. Exp. Biol. 216 (Pt 18), 3504-3513. https://doi.org/10.1242/jeb. 087635 .

Bill, B.R., Petzold, A.M., Clark, K.J., Schimmenti, L.A., Ekker, S.C., 2009. A Primer for Morpholino Use in Zebrafish. Zebrafish 6 (1), 69-77. https://doi.org/10.1089/zeb. 2008.0555.

Blank, M., Guerim, L.D., Cordeiro, R.F., Vianna, M.R.M., 2009. A one-trial inhibitory avoidance task to zebrafish: rapid acquisition of an NMDA-dependent long-term memory. Neurobiol. Learn. Mem. 92 (4), 529-534. https://doi.org/10.1016/j.nlm. 2009.07.001.

Blizinsky, K.D., Diaz-Castro, B., Forrest, M.P., Schürmann, B., Bach, A.P., Martin-deSaavedra, M.D., Wang, L.; Csernansky, J.G., Duan, J., Penzes, P., 2016. Reversal of dendritic phenotypes in $16 \mathrm{p} 11.2$ microduplication mouse model neurons by pharmacological targeting of a network hub. Proc. Natl. Acad. Sci. U. S. A. 113 (30), 8520-8525. https://doi.org/10.1073/pnas.1607014113.

Boyd, P.J., Cunliffe, V.T., Roy, S., Wood, J.D., 2015. Sonic hedgehog functions upstream of disrupted-in-schizophrenia 1 (disc1): implications for mental illness. Biol. Open 4 (10), 1336-1343. https://doi.org/10.1242/bio.012005.

Brauns, S., Gollub, R.L., Roffman, J.L., Yendiki, A., Ho, B.-C., Wassink, T.H., Heinz, A., Ehrlich, S., 2011. DISC1 is associated with cortical thickness and neural efficiency. NeuroImage 57 (4), 1591-1600. https://doi.org/10.1016/j.neuroimage.2011.05. 058 .

Bubeníková-Valešová, V., Horáček, J., Vrajová, M., Höschl, C., 2008. Models of schizophrenia in humans and animals based on inhibition of NMDA receptors. Neurosci. Biobehav. Rev. 32 (5), 1014-1023. https://doi.org/10.1016/j.neubiorev.2008.03. 012.

Burgess, H.A., Granato, M., 2007. Sensorimotor gating in larval zebrafish. J. Neurosci. 27 (18), 4984-4994. https://doi.org/10.1523/JNEUROSCI.0615-07.2007.

Burgstaller, J., Hindinger, E., Gesierich, B., Baier, H., 2019a. Light-sheet imaging and graph-theoretical analysis of antidepressant action in the larval zebrafish brain netgraph-theoretical analysis of antidepressant action in the 1 .
work. BioRxiv, 618843. https://doi.org/10.1101/618843.

Burgstaller, J., Hindinger, E., Gesierich, B., Baier, H., 2019b. Light-sheet Imaging and Graph-theoretical Analysis of Antidepressant Action in the Larval Zebrafish Brain Network (n.d.). pp. 26.

Callicott, J.H., Straub, R.E., Pezawas, L., Egan, M.F., Mattay, V.S., Hariri, A.R., Verchinski, B.A., Meyer-Lindenberg, A., Balkissoon, R., Kolachana, B., Goldberg, T.E., Weinberger, D.R., 2005. Variation in DISC1 affects hippocampal structure and function and increases risk for schizophrenia. Proc. Natl. Acad. Sci. U. S. A. 102 (24), 8627-8632. https://doi.org/10.1073/pnas.0500515102.

Cannon, T.D., Hennah, W., van Erp, T.G.M., Thompson, P.M., Lonnqvist, J., Huttunen, M., Gasperoni, T., Tuulio-Henriksson, A., Pirkola, T., Toga, A.W., Kaprio, J., Mazziotta, J., Peltonen, L., 2005. Association of DISC1/TRAX haplotypes with schizophrenia, reduced prefrontal gray matter, and impaired short- and long-term memory. Arch. reduced prefrontal gray matter, and impaired short- and long-term memory. Arch.
Gen. Psychiatry 62 (11), 1205-1213. https://doi.org/10.1001/archpsyc.62.11.1205.

Chen, J., Patel, R., Friedman, T.C., Jones, K.S., 2010. The behavioral and pharmacological actions of NMDA receptor antagonism are conserved in zebrafish larvae. Int. J. Comp Psychol. 23 (1), 82-90.

Cheng, Y.C., Scotting, P.J., Hsu, L.S., Lin, S.J., Shih, H.Y., Hsieh, F.Y., Wu, H.L., Tsao, C.L., Shen, C.J., 2013. Zebrafish rgs4 is essential for motility and axonogenesis mediated by Akt signaling. Cell. Mol. Life Sci. 70 (5), 935-950. https://doi.org/10.1007/ s00018-012-1178-z.

Chesworth, R., Yulyaningsih, E., Cappas, E., Arnold, J., Sainsbury, A., Karl, T., 2012. The response of neuregulin 1 mutant mice to acute restraint stress. Neurosci. Lett. 515 (1), 82-86. https://doi.org/10.1016/j.neulet.2012.03.024.

Clapcote, S.J., Lipina, T.V., Millar, J.K., Mackie, S., Christie, S., Ogawa, F., Lerch, J.P., Trimble, K., Uchiyam, a M., Sakuraba, Y., Kaneda, H., Shiroishi, T., Houslay, M.D., Henkelman, R.M., Sled, J.G., Gondo, Y., Porteous, D.J., Roder, J.C., 2007. Behavioral phenotypes of Disc1 missense mutations in mice. Neuron 54 (3), 387-402. https:// doi.org/10.1016/j.neuron.2007.04.015.

Cognato, G., de, P., Bortolotto, J.W., Blazina, A.R., Christoff, R.R., Lara, D.R., Vianna, M.R., Bonan, C.D., 2012. Y-Maze memory task in zebrafish (Danio rerio): the role of glutamatergic and cholinergic systems on the acquisition and consolidation periods. Neurobiol. Learn. Mem. 98 (4), 321-328. https://doi.org/10.1016/j.nlm.2012.09. 008

Costain, G., Lionel, A.C., Merico, D., Forsythe, P., Russell, K., Lowther, C., Yuen, T., Husted, J., Stavropoulos, D.J., Speevak, M., Chow, E.W., Marshall, C.R., Scherer, S.W., Bassett, A.S., 2013. Pathogenic rare copy number variants in community-based schizophrenia suggest a potential role for clinical microarrays. Hum. Mol. Genet. 22 (22), 4485-4501. https://doi.org/10.1093/hmg/ddt297.

Cross-Disorder Group of the Psychiatric Genomics Consortium, 2013. Identification of risk loci with shared effects on five major psychiatric disorders: a genome-wide analysis. Lancet (London, England) 381 (9875), 1371-1379. https://doi.org/10. 1016/S0140-6736(12)62129-1.

de Bartolomeis, A., Sarappa, C., Buonaguro, E.F., Marmo, F., Eramo, A., Tomasetti, C., Iasevoli, F., 2013. Different effects of the NMDA receptor antagonists ketamine, MK801 , and memantine on postsynaptic density transcripts and their topography: role of Homer signaling, and implications for novel antipsychotic and pro-cognitive targets in psychosis. Prog. Neuropsychopharmacol. Biol. Psychiatry 46, 1-12. https://doi. org/10.1016/j.pnpbp.2013.06.010.

De Rienzo, G., Bishop, J.A., Mao, Y., Pan, L., Ma, T.P., Moens, C.B., Tsai, L.H., Sive, H. 2011. Disc1 regulates both $\beta$-catenin-mediated and noncanonical Wnt signaling during vertebrate embryogenesis. FASEB J. 25 (12), 4184-4197. https://doi.org/10. 1096/fj.11-186239.

Degenhardt, F., Heinemann, B., Strohmaier, J., Pfohl, M.A., Giegling, I., Hofmann, A., Ludwig, K.U., Witt, S.H., Ludwig, M., Forstner, A.J., Albus, M., Schwab, S.G., Borrmann-Hassenbach, M., Lennertz, L., Wagner, M., Hoffmann, P., Rujescu, D., Maier, W., Cichon, S., Rietschel, M., Nöthen, M.M., 2016. Identification of rare variants in KCTD13 at the schizophrenia risk locus 16p11.2. Psychiatr. Genet. 26 (6), 293-296. https://doi.org/10.1097/YPG.0000000000000145.

Deng, C., Dean, B., 2013. Mapping the pathophysiology of schizophrenia: interactions between multiple cellular pathways. Front. Cell. Neurosci. 7. https://doi.org/10. 3389/fncel.2013.00238.

Desbonnet, L., O’Tuathaigh, C., Clarke, G., O’Leary, C., Petit, E., Clarke, N., Tighe, O., Lai, D., Harvey, R., Cryan, J.F., Dinan, T.G., Waddington, J.L., 2012. Phenotypic effects of repeated psychosocial stress during adolescence in mice mutant for the schizophrenia risk gene neuregulin-1: A putative model of gene $\times$ environment interaction. Brain Behav. Immun. 26 (4), 660-671. https://doi.org/10.1016/j.bbi.2012.02.010.

Drerup, C.M., Wiora, H.M., Topczewski, J., Morris, J.A., 2009. Disc1 regulates foxd3 and sox10 expression, affecting neural crest migration and differentiation. Development sox10 expression, affecting neural crest migration and differentiation. Development
(Cambridge, England) 136 (15), 2623-2632. https://doi.org/10.1242/dev.030577.

Duff, B.J., Macritchie, K.A.N., Moorhead, T.W.J., Lawrie, S.M., Blackwood, D.H.R., 2013. Human brain imaging studies of DISC1 in schizophrenia, bipolar disorder and depression: a systematic review. Schizophr. Res. 147 (1), 1-13. https://doi.org/10. 1016/j.schres.2013.03.015.

Eachus, H., Bright, C., Cunliffe, V.T., Placzek, M., Wood, J.D., Watt, P.J., 2017. Disruptedin-Schizophrenia-1 is essential for normal hypothalamic-pituitary-interrenal (HPI) axis function. Hum. Mol. Genet. 26 (11), 1992-2005. https://doi.org/10.1093/hmg/ ddx076.

Echevarria, D.J., Hammack, C.M., Pratt, D.W., Hosemann, J.D., 2008. A novel behavioral test battery to assess global drug effects using the zebrafish. Int. J. Comp. Psychol. 21 (1), 19-34.

Failla, P., Romano, C., Alberti, A., Vasta, A., Buono, S., Castiglia, L., Luciano, D., Di Benedetto, D., Fichera, M., Galesi, O., 2007. Schizophrenia in a patient with subtelomeric duplication of chromosome 22q. Clin. Genet. 71 (6), 599-601. https://doi org/10.1111/j.1399-0004.2007.00819.x.

Fish, B., Marcus, J., Hans, S.L., Auerbach, J.G., Perdue, S., 1992. Infants at risk for schizophrenia: sequelae of a genetic neurointegrative defect. A review and replication analysis of pandysmaturation in the Jerusalem Infant Development Study. Arch. Gen. Psychiatry 49 (3), 221-235.

Fleming, A., Diekmann, H., Goldsmith, P., 2013. Functional characterisation of the maturation of the blood-brain barrier in larval zebrafish. PLoS One 8 (10), e77548. https://doi.org/10.1371/journal.pone.0077548.

Formella, I., Scott, E.K., Burne, T.H.J., Harms, L.R., Liu, P.-Y., Turner, K.M., Cui, X., Eyles, D.W., 2012. Transient knockdown of tyrosine hydroxylase during development has persistent effects on behaviour in adult zebrafish (Danio rerio). PLoS One 7 (8), e42482. https://doi.org/10.1371/journal.pone.0042482.

Friedrich, R.W., Genoud, C., Wanner, A.A., 2013. Analyzing the structure and function of 
neuronal circuits in zebrafish. Front. Neural Circuits 7, 71. https://doi.org/10.3389/ fncir.2013.00071.

Fromer, M., Roussos, P., Sieberts, S.K., Johnson, J.S., Kavanagh, D.H., Perumal, T.M., Ruderfer, D.M., Oh, E.C., Topol, A., Shah, H.R., Klei, L.L., Kramer, R., Pinto, D., Gümüş, Z.H., Cicek, A.E., Dang, K.K., Browne, A., Lu, C., Xie, L., Readhead, B., Stahl, E.A., Xiao, J., Parvizi, M., Hamamsy, T., Fullard, J.F., Wang, Y.-C., Mahajan, M.C., Derry, J.M.J., Dudley, J.T., Hemby, S.E., Logsdon, B.A., Talbot, K., Raj, T., Bennett, D.A., De Jager, P.L., Zhu, J., Zhang, B., Sullivan, P.F., Chess, A., Purcell, S.M., Shinobu, L.A., Mangravite, L.M., Toyoshiba, H., Gur, R.E., Hahn, C.-G., Lewis, D.A., Haroutunian, V., Peters, M.A., Lipska, B.K., Buxbaum, J.D., Schadt, E.E., Hirai, K., Roeder, K., Brennand, K.J., Katsanis, N., Domenici, E., Devlin, B., Sklar, P., 2016. Gene expression elucidates functional impact of polygenic risk for schizophrenia. Nat. Neurosci. 19, 1442-1453. https://doi.org/10.1038/nn.4399.

Gahtan, E., Sankrithi, N., Campos, J.B., O'Malley, D.M., 2002. Evidence for a widespread brain stem escape network in larval zebrafish. J. Neurophysiol. 87, 608-614. https:// doi.org/10.1152/jn.00596.2001.

Garlick, P.J., 2006. Toxicity of methionine in humans. J. Nutr. 136, 1722S-1725S. https://doi.org/10.1093/jn/136.6.1722S.

Gaspary, K.V., Reolon, G.K., Gusso, D., Bonan, C.D., 2018. Novel object recognition and object location tasks in zebrafish: influence of habituation and NMDA receptor antagonism. Neurobiol. Learn. Mem. 155, 249-260. https://doi.org/10.1016/j.nlm. 2018.08.005.

Gauthier, J., Champagne, N., Lafrenière, R.G., Xiong, L., Spiegelman, D., Brustein, E., Lapointe, M., Peng, H., Côté, M., Noreau, A., Hamdan, F.F., Addington, A.M., Rapoport, J.L., Delisi, L.E., Krebs, M.-O., Joober, R., Fathalli, F., Mouaffak, F., Haghighi, A.P., Néri, C., Dubé, M.-P., Samuels, M.E., Marineau, C., Stone, E.A., Awadalla, P., Barker, P.A., Carbonetto, S., Drapeau, P., Rouleau, G.A., Team, S.2D., 2010. De novo mutations in the gene encoding the synaptic scaffolding protein SHANK3 in patients ascertained for schizophrenia. Proc. Natl. Acad. Sci. U. S. A. 107, 7863-7868. https://doi.org/10.1073/pnas.0906232107.

Gerlai, R., 2016. Chapter 17 - "learning and memory in zebrafish (Danio rerio). In: Detrich, H.W., Westerfield, M., Zon, L.I. (Eds.), Methods in Cell Biology, The Zebrafish. Academic Press, pp. 551-586. https://doi.org/10.1016/bs.mcb.2016.02. 005 .

Giacomotto, J., Carroll, A.P., Rinkwitz, S., Mowry, B., Cairns, M.J., Becker, T.S., 2016. Developmental suppression of schizophrenia-associated miR-137 alters sensorimotor function in zebrafish. Transl. Psychiatry 6, e818. https://doi.org/10.1038/tp. 2016.88 .

Golzio, C., Willer, J., Talkowski, M.E., Oh, E.C., Taniguchi, Y., Jacquemont, S., Reymond, A., Sun, M., Sawa, A., Gusella, J.F., Kamiya, A., Beckmann, J.S., Katsanis, N., 2012 KCTD13 is a major driver of mirrored neuroanatomical phenotypes of the $16 \mathrm{p} 11.2$ copy number variant. Nature 485, 363-367. https://doi.org/10.1038/nature11091.

Grabrucker, S., Proepper, C., Mangus, K., Eckert, M., Chhabra, R., Schmeisser, M.J. Boeckers, T.M., Grabrucker, A.M., 2014. The PSD protein ProSAP2/Shank3 displays synapto-nuclear shuttling which is deregulated in a schizophrenia-associated mutation. Exp. Neurol. 253, 126-137. https://doi.org/10.1016/j.expneurol.2013.12.015

Green, J., Collins, C., Kyzar, E.J., Pham, M., Roth, A., Gaikwad, S., Cachat, J., Stewart, A.M., Landsman, S., Grieco, F., Tegelenbosch, R., Noldus, L.P.J.J., Kalueff, A.V., 2012. Automated high-throughput neurophenotyping of zebrafish social behavior. J. Neurosci. Methods 210, 266-271. https://doi.org/10.1016/j.jneumeth.2012.07.017.

Grillet, N., Pattyn, A., Contet, C., Kieffer, B.L., Goridis, C., Brunet, J.-F., 2005. Generation and characterization of Rgs4 mutant mice. Mol. Cell. Biol. 25, 4221-4228. https:// doi.org/10.1128/MCB.25.10.4221-4228.2005.

Grossman, L., Utterback, E., Stewart, A., Gaikwad, S., Chung, K.M., Suciu, C., Wong, K., Elegante, M., Elkhayat, S., Tan, J., Gilder, T., Wu, N., Dileo, J., Cachat, J., Kalueff, A.V., 2010. Characterization of behavioral and endocrine effects of LSD on zebrafish. Behav. Brain Res. 214, 277-284. https://doi.org/10.1016/j.bbr.2010.05.039.

Guna, A., Butcher, N.J., Bassett, A.S., 2015. Comparative mapping of the 22q11.2 deletion region and the potential of simple model organisms. J. Neurodev. Disord. 7, 18 . https://doi.org/10.1186/s11689-015-9113-x.

Guo, S., 2009. Using zebrafish to assess the impact of drugs on neural development and function. Expert Opin. Drug Discov. 4, 715-726. https://doi.org/10.1517/ 17460440902988464

Guo, S., Wagle, M., Mathur, P., 2012. Toward molecular genetic dissection of neural circuits for emotional and motivational behaviors. Dev. Neurobiol. 72, 358-365. https://doi.org/10.1002/dneu.20927.

Gusev, A., Mancuso, N., Won, H., Kousi, M., Finucane, H.K., Reshef, Y., Song, L., Safi, A., Schizophrenia Working Group of the Psychiatric Genomics Consortium, McCarroll, S., Neale, B.M., Ophoff, R.A., O’Donovan, M.C., Crawford, G.E., Geschwind, D.H., Katsanis, N., Sullivan, P.F., Pasaniuc, B., Price, A.L., 2018. Transcriptome-wide association study of schizophrenia and chromatin activity yields mechanistic disease insights. Nat. Genet. 50, 538-548. https://doi.org/10.1038/s41588-018-0092-1.

Haesemeyer, M., Schier, A.F., 2015. The study of psychiatric disease genes and drugs in zebrafish. Curr. Opin. Neurobiol. 0, 122-130. https://doi.org/10.1016/j.conb.2014. 12.002

Halberstadt, A.L., Powell, S.B., Geyer, M.A., 2013. Role of the 5-HT2A receptor in the locomotor hyperactivity produced by phenylalkylamine hallucinogens in mice. Neuropharmacology 70, 218-227. https://doi.org/10.1016/j.neuropharm.2013.01. 014.

Ham, S., Kim, T.K., Chung, S., Im, H.-I., 2017. Drug abuse and psychosis: new insights into drug-induced psychosis. Exp. Neurobiol. 26, 11-24. https://doi.org/10.5607/en. 2017.26.1.11.

Harrop, C., Trower, P., 2001. Why does schizophrenia develop at late adolescence? Clin. Psychol. Rev. 21, 241-265. https://doi.org/10.1016/S0272-7358(99)00047-1.

Hashimoto, R., Numakawa, T., Ohnishi, T., Kumamaru, E., Yagasaki, Y., Ishimoto, T., Mori, T., Nemoto, K., Adachi, N., Izumi, A., Chiba, S., Noguchi, H., Suzuki, T., Iwata, N., Ozaki, N., Taguchi, T., Kamiya, A., Kosuga, A., Tatsumi, M., Kamijima, K., Weinberger, D.R., Sawa, A., Kunugi, H., 2006. Impact of the DISC1 Ser704Cys polymorphism on risk for major depression, brain morphology and ERK signaling. Hum. Mol. Genet. 15, 3024-3033. https://doi.org/10.1093/hmg/ddl244.
Hill, A., Howard, C.V., Strahle, U., Cossins, A., 2003. Neurodevelopmental defects in zebrafish (Danio rerio) at environmentally relevant dioxin (TCDD) concentrations. Toxicol. Sci. 76, 392-399. https://doi.org/10.1093/toxsci/kfg241.

Hinsch, K., Zupanc, G.K.H., 2007. Generation and long-term persistence of new neuron in the adult zebrafish brain: a quantitative analysis. Neuroscience 146, 679-696. https://doi.org/10.1016/j.neuroscience.2007.01.071

Howe, K., Clark, M.D., Torroja, C.F., Torrance, J., Berthelot, C., Muffato, M., Collins, J.E., Humphray, S., McLaren, K., Matthews, L., McLaren, S., Sealy, I., Caccamo, M., Churcher, C., Scott, C., Barrett, J.C., Koch, R., Rauch, G.-J., White, S., Chow, W. Kilian, B., Quintais, L.T., Guerra-Assunção, J.A., Zhou, Y., Gu, Y., Yen, J., Vogel, J.-H., Eyre, T., Redmond, S., Banerjee, R., Chi, J., Fu, B., Langley, E., Maguire, S.F., Laird, G.K., Lloyd, D., Kenyon, E., Donaldson, S., Sehra, H., Almeida-King, J., Loveland, J., Trevanion, S., Jones, M., Quail, M., Willey, D., Hunt, A., Burton, J., Sims, S., McLay, K., Plumb, B., Davis, J., Clee, C., Oliver, K., Clark, R., Riddle, C., Elliott, D., Threadgold, G., Harden, G., Ware, D., Begum, S., Mortimore, B., Kerry, G., Heath, P., Phillimore, B., Tracey, A., Corby, N., Dunn, M., Johnson, C., Wood, J., Clark, S., Phillimore, B., Tracey, A., Corby, N., Dunn, M., Johnson, C., Wood, J., Clark, S., Stevens, C., Harley, J., Holt, K., Panagiotidis, G., Lovell, J., Beasley, H., Henderson, C., Gordon, D., Auger, K., Wright, D., Collins, J., Raisen, C., Dyer, L., Leung, K., Robertson, L., Ambridge, K., Leongamornlert, D., McGuire, S., Gilderthorp, R., Griffiths, C., Manthravadi, D., Nichol, S., Barker, G., Whitehead, S., Kay, M., Brown, J., Murnane, C., Gray, E., Humphries, M., Sycamore, N., Barker, D., Saunders, D., Wallis, J., Babbage, A., Hammond, S., Mashreghi-Mohammadi, M., Barr, L., Martin, S., Wray, P., Ellington, A., Matthews, N., Ellwood, M., Woodmansey, R., Clark, G., Cooper, J.D., Tromans, A., Grafham, D., Skuce, C., Pandian, R., Andrews, R., Harrison, E., Kimberley, A., Garnett, J., Fosker, N., Hall, R., Garner, P., Kelly, D., Bird, C., Palmer, S., Gehring, I., Berger, A., Dooley, C.M., Ersan-Ürün, Z., Eser, C., Geiger, H., Geisler, M., Karotki, L., Kirn, A., Konantz, J., Konantz, M., Oberländer, M., Rudolph-Geiger, S., Teucke, M., Lanz, C., Raddatz, G., Osoegawa, K., Zhu, B., Rapp, A., Widaa, S., Langford, C., Yang, F., Schuster, S.C., Carter, N.P., Harrow, J., Ning, Z., Herrero, J., Searle, S.M.J., Enright, A., Geisler, R., Plasterk, R.H.A., Lee, C., Westerfield, M., de Jong, P.J., Zon, L.I., Postlethwait, J.H., Nüsslein-Volhard, C., Hubbard, T.J.P., Crollius, H.R., Rogers, J., Stemple, D.L., 2013. The zebrafish reference genome sequence and its relationship to the human genome. Nature 496, 498-503. https://doi.org/10.1038/nature12111.

Huertas-Vazquez, A., Teodorescu, C., Reinier, K., Uy-Evanado, A., Chugh, H., Jerger, K., Ayala, J., Gunson, K., Jui, J., Newton-Cheh, C., Albert, C.M., Chugh, S.S., 2013. A common missense variant in the Neuregulin1 gene is associated with both schizophrenia and sudden cardiac death. Heart Rhythm 10, 994-998. https://doi.org/10 1016/j.hrthm.2013.03.020.

Irons, T.D., MacPhail, R.C., Hunter, D.L., Padilla, S., 2010. Acute neuroactive drug exposures alter locomotor activity in larval zebrafish. Neurotoxicol. Teratol. 32, 84-90. https://doi.org/10.1016/j.ntt.2009.04.066.

Jarvis, E.D., Güntürkün, O., Bruce, L., Csillag, A., Karten, H., Kuenzel, W., Medina, L., Paxinos, G., Perkel, D.J., Shimizu, T., Striedter, G., Wild, J.M., Ball, G.F., Dugas-Ford, J., Durand, S.E., Hough, G.E., Husband, S., Kubikova, L., Lee, D.W., Mello, C.V., Powers, A., Siang, C., Smulders, T.V., Wada, K., White, S.A., Yamamoto, K., Yu, J., Reiner, A., Butler, A.B., Avian Brain Nomenclature Consortium, 2005. Avian brains and a new understanding of vertebrate brain evolution. Nat. Rev. Neurosci. 6, 151-159. https://doi.org/10.1038/nrn1606.

Jeans, A.F., Oliver, P.L., Johnson, R., Capogna, M., Vikman, J., Molnár, Z., Babbs, A. Partridge, C.J., Salehi, A., Bengtsson, M., Eliasson, L., Rorsman, P., Davies, K.E., 2007. A dominant mutation in Snap25 causes impaired vesicle trafficking, sensorimotor gating, and ataxia in the blind-drunk mouse. Proc. Natl. Acad. Sci. U.S.A. 104, 2431-2436. https://doi.org/10.1073/pnas.0610222104.

Jeong, J.-Y., Kwon, H.-B., Ahn, J.-C., Kang, D., Kwon, S.-H., Park, J.A., Kim, K.-W., 2008 Functional and developmental analysis of the blood-brain barrier in zebrafish. Brai Res. Bull. 75, 619-628. https://doi.org/10.1016/j.brainresbull.2007.10.043.

Johnson, R.D., Oliver, P.L., Davies, K.E., 2008. SNARE proteins and schizophrenia: linking synaptic and neurodevelopmental hypotheses. Acta Biochim. Pol. 55, 619-628.

Jones, C., Watson, D., Fone, K., 2011. Animal models of schizophrenia. Br. J. Pharmacol. 164, 1162-1194. https://doi.org/10.1111/j.1476-5381.2011.01386.x.

Jones, L.J., Norton, W.H.J., 2015. Using zebrafish to uncover the genetic and neural basis of aggression, a frequent comorbid symptom of psychiatric disorders. Behavioural Brain Research, SI: Neuropsychiatric Spectra 276, 171-180. https://doi.org/10. 1016/j.bbr.2014.05.055.

Kalueff, A.V., Stewart, A.M., Gerlai, R., 2014. Zebrafish as an emerging model for studying complex brain disorders. Trends Pharmacol. Sci. 35, 63-75. https://doi.org/ 10.1016/j.tips.2013.12.002.

Karl, T., Duffy, L., Scimone, A., Harvey, R.P., Schofield, P.R., 2007. Altered motor activity, exploration and anxiety in heterozygous neuregulin 1 mutant mice: implications for understanding schizophrenia. Genes Brain Behav. 6, 677-687. https://doi. tions for understanding schizophrenia. Gen
org/10.1111/j.1601-183X.2006.00298.x.

Kasap, M., Rajani, V., Rajani, J., Dwyer, D.S., 2018. Surprising conservation of schizophrenia risk genes in lower organisms reflects their essential function and the evolution of genetic liability. Schizophr. Res. 202, 120-128. https://doi.org/10.1016/j. schres.2018.07.017.

Kawakami, K., 2005. Transposon tools and methods in zebrafish. Dev. Dyn. 234, 244-254. https://doi.org/10.1002/dvdy.20516.

Kawakami, K., Koga, A., Hori, H., Shima, A., 1998. Excision of the Tol2 transposable element of the medaka fish, Oryzias latipes, in zebrafish, Danio rerio. Gene 225, 17-22. https://doi.org/10.1016/S0378-1119(98)00537-X.

Kim, J., Horti, A.G., Mathews, W.B., Pogorelov, V., Valentine, H., Brasic, J.R., Holt, D.P., Ravert, H.T., Dannals, R.F., Zhou, L., Jedynak, B., Kamiya, A., Pletnikov, M.V., Wong D.F., 2015. Quantitative multi-modal brain autoradiography of glutamatergic, dopaminergic, cannabinoid, and nicotinic receptors in mutant Disrupted-InSchizophrenia-1 (DISC1) mice. Mol. Imaging Biol. 17, 355-363. https://doi.org/10. 1007/s11307-014-0786-4.

Kimmel, C.B., Ballard, W.W., Kimmel, S.R., Ullmann, B., Schilling, T.F., 1995. Stages of embryonic development of the zebrafish. Dev. Dyn. 203, 253-310. https://doi.org/ 
10.1002/aja.1002030302

Kirov, G., Pocklington, A.J., Holmans, P., Ivanov, D., Ikeda, M., Ruderfer, D., Moran, J., Chambert, K., Toncheva, D., Georgieva, L., Grozeva, D., Fjodorova, M., Wollerton, R., Rees, E., Nikolov, I., van de Lagemaat, L.N., Bayés, A., Fernandez, E., Olason, P.I. Böttcher, Y., Komiyama, N.H., Collins, M.O., Choudhary, J., Stefansson, K. Stefansson, H., Grant, S.G.N., Purcell, S., Sklar, P., O’Donovan, M.C., Owen, M.J., 2012. De novo CNV analysis implicates specific abnormalities of postsynaptic signalling complexes in the pathogenesis of schizophrenia. Mol. Psychiatry 17, 142-153. https://doi.org/10.1038/mp.2011.154

Kohashi, T., Oda, Y., 2008a. Initiation of Mauthner- or non-Mauthner-mediated fast escape evoked by different modes of sensory input. J. Neurosci. 28, 10641-10653. https://doi.org/10.1523/JNEUROSCI.1435-08.2008.

Kohashi, T., Oda, Y., 2008b. Initiation of Mauthner- or non-Mauthner-mediated fast escape evoked by different modes of sensory input. J. Neurosci. 28, 10641-10653. https://doi.org/10.1523/JNEUROSCI.1435-08.2008.

Kokel, D., Peterson, R.T., 2008. Chemobehavioural phenomics and behaviour-based psychiatric drug discovery in the zebrafish. Brief. Funct. Genomic. Proteomic. 7, 483-490. https://doi.org/10.1093/bfgp/eln040.

Kozol, R.A., Cukier, H.N., Zou, B., Mayo, V., De Rubeis, S., Cai, G., Griswold, A.J., Whitehead, P.L., Haines, J.L., Gilbert, J.R., Cuccaro, M.L., Martin, E.R., Baker, J.D., Buxbaum, J.D., Pericak-Vance, M.A., Dallman, J.E., 2015. Two knockdown models of the autism genes SYNGAP1 and SHANK3 in zebrafish produce similar behavioral phenotypes associated with embryonic disruptions of brain morphogenesis. Hum. phenotypes associated with embryonic disruptions of brain morphogen

Kyzar, E., Stewart, A.M., Landsman, S., Collins, C., Gebhardt, M., Robinson, K., Kalueff, A.V., 2013. Behavioral effects of bidirectional modulators of brain monoamines reserpine and d-amphetamine in zebrafish. Brain Res. 1527, 108-116. https://doi.org/ 10.1016/j.brainres.2013.06.033.

Kyzar, E.J., Collins, C., Gaikwad, S., Green, J., Roth, A., Monnig, L., El-Ounsi, M., Davis, A., Freeman, A., Capezio, N., Stewart, A.M., Kalueff, A.V., 2012. Effects of hallucinogenic agents mescaline and phencyclidine on zebrafish behavior and physiology. Prog. Neuropsychopharmacol. Biol. Psychiatry 37, 194-202. https://doi.org/10. 1016/j.pnpbp.2012.01.003.

Lautar, S.L., Rojas, C., Slusher, B.S., Wozniak, K.M., Wu, Y., Thomas, A.G., Waldon, D., Li, W., Ferraris, D., Belyakov, S., 2005. DPP IV inhibitor blocks mescaline-induced scratching and amphetamine-induced hyperactivity in mice. Brain Res. 1048, 177-184. https://doi.org/10.1016/j.brainres.2005.04.069.

Lawson, N.D., Wolfe, S.A., 2011. Forward and reverse genetic approaches for the analysis of vertebrate development in the zebrafish. Dev. Cell 21, 48-64. https://doi.org/10 1016/j.devcel.2011.06.007.

Lee, F.H.F., Fadel, M.P., Preston-Maher, K., Cordes, S.P., Clapcote, S.J., Price, D.J., Roder, J.C., Wong, A.H.C., 2011. Disc1 point mutations in mice affect development of the cerebral cortex. J. Neurosci. 31, 3197-3206. https://doi.org/10.1523/JNEUROSCI. 4219-10.2011.

Leung, L.C., Wang, G.X., Mourrain, P., 2013. Imaging zebrafish neural circuitry from whole brain to synapse. Front. Neural Circuits 7. https://doi.org/10.3389/fncir.2013. 00076.

Levitt, P., Ebert, P., Mirnics, K., Nimgaonkar, V.L., Lewis, D.A., 2006. Making the case for a candidate vulnerability gene in schizophrenia: convergent evidence for regulator of G-protein signaling 4 (RGS4). Biol. Psychiatry 60, 534-537. https://doi.org/10. 1016/j.biopsych.2006.04.028.

Li, F, Lin, J., Liu, X., Li, W., Ding, Y, Zhang, Y, Zhou, S, Guo, N., Li, Q, 2018. Characterization of the locomotor activities of zebrafish larvae under the influence of various neuroactive drugs. Ann. Transl. Med. 6, 173. https://doi.org/10.21037/atm. 2018.04.25.

Lieschke, G.J., Currie, P.D., 2007. Animal models of human disease: zebrafish swim into view. Nat. Rev. Genet. 8, 353-367. https://doi.org/10.1038/nrg2091.

Light, S.E.W., Jontes, J.D., 2019. Multiplane calcium imaging reveals disrupted development of network topology in zebrafish pcdh19 mutants. eNeuro 6. https://doi.org/ 10.1523/ENEURO.0420-18.2019.

Lipina, T.V., Kaidanovich-Beilin, O., Patel, S., Wang, M., Clapcote, S.J., Liu, F., Woodgett, J.R., Roder, J.C., 2011. Genetic and pharmacological evidence for sCHIZOPHRENIA-
rELATED DISC1 interaction with GSK-3. Synapse 65, 234-248. https://doi.org/10. rELATED DISC1

Lipina, T.V., Wang, M., Liu, F., Roder, J.C., 2012. Synergistic interactions between PDE4B and GSK-3: DISC1 mutant mice. Neuropharmacology 62, 1252-1262. https://doi. org/10.1016/j.neuropharm.2011.02.020

Liu, C.-X., Li, C.-Y., Hu, C.-C., Wang, Y., Lin, J., Jiang, Y.-H., Li, Q., Xu, X., 2018. CRISPR/ Cas9-induced shank3b mutant zebrafish display autism-like behaviors. Mol. Autism 9, 23. https://doi.org/10.1186/s13229-018-0204-x.

Liu, X., Guo, N., Lin, J., Zhang, Y., Chen, X.Q., Li, S., He, L., Li, Q., 2014. Strain-dependent differential behavioral responses of zebrafish larvae to acute MK-801 treatment. Pharmacol. Biochem. Behav. 127, 82-89. https://doi.org/10.1016/j.pbb.2014.11. Pharm.

López-Schier, H., 2019. Neuroplasticity in the acoustic startle reflex in larval zebrafish. Curr. Opin. Neurobiol. 54, 134-139. https://doi.org/10.1016/j.conb.2018.10.004

Loviglio, M.N., Arbogast, T., Jønch, A.E., Collins, S.C., Popadin, K., Bonnet, C.S., Giannuzzi, G., Maillard, A.M., 16p11.2 Consortium, Jacquemont, S., Yalcin, B., Katsanis, N., Golzio, C., Reymond, A., 2017. The immune signaling adaptor LAT contributes to the neuroanatomical phenotype of 16p11.2 BP2-BP3 CNVs. Am. J. Hum. Genet. 101, 564-577. https://doi.org/10.1016/j.ajhg.2017.08.016.

Maaswinkel, H., Zhu, L., Weng, W., 2013. Assessing social engagement in heterogeneous groups of zebrafish: a new paradigm for autism-like behavioral responses. PLoS One 8, e75955. https://doi.org/10.1371/journal.pone.0075955.

Mahabir, S., Chatterjee, D., Buske, C., Gerlai, R., 2013. Maturation of shoaling in two zebrafish strains: a behavioral and neurochemical analysis. Behav. Brain Res. 247, 1-8. https://doi.org/10.1016/j.bbr.2013.03.013.

Mäki, P., Veijola, J., Jones, P.B., Murray, G.K., Koponen, H., Tienari, P., Miettunen, J., Tanskanen, P., Wahlberg, K.-E., Koskinen, J., Lauronen, E., Isohanni, M., 2005. Predictors of schizophrenia—a review. Br. Med. Bull. 73-74, 1-15. https://doi.org/ 10.1093/bmb/ldh046.

Manahan-Vaughan, D., von Haebler, D., Winter, C., Juckel, G., Heinemann, U., 2008. A single application of MK801 causes symptoms of acute psychosis, deficits in spatial memory, and impairment of synaptic plasticity in rats. Hippocampus 18, 125-134. memory, and impairment of synaptic

Marcotte, E.R., Pearson, D.M., Srivastava, L.K., 2001. Animal models of schizophrenia: critical review. J. Psychiatry Neurosci. 26, 395-410.

Menezes, F.P., Kist, L.W., Bogo, M.R., Bonan, C.D., Da Silva, R.S., 2015. Evaluation of Age-Dependent Response to NMDA Receptor Antagonism in Zebrafish. Zebrafish 12, 137-143. https://doi.org/10.1089/zeb.2014.1018.

Michelotti, P., Quadros, V.A., Pereira, M.E., Rosemberg, D.B., 2018. Ketamine modulates aggressive behavior in adult zebrafish. Neurosci. Lett. 684, 164-168. https://doi.org/ 10.1016/j.neulet.2018.08.009.

Migliavacca, E., Golzio, C., Männik, K., Blumenthal, I., Oh, E.C., Harewood, L., Kosmicki, J.A., Loviglio, M.N., Giannuzzi, G., Hippolyte, L., Maillard, A.M., Alfaiz, A.A., 16p11.2 European Consortium, van Haelst, M.M., Andrieux, J., Gusella, J.F., Daly, M.J., Beckmann, J.S., Jacquemont, S., Talkowski, M.E., Katsanis, N., Reymond, A., 2015 . A potential contributory role for ciliary dysfunction in the $16 \mathrm{p} 11.2600 \mathrm{~kb}$ BP4 BP5 pathology. Am. J. Hum. Genet. 96, 784-796. https://doi.org/10.1016/j.ajhg. 2015.04.002.

Miller, N., Gerlai, R., 2007. Quantification of shoaling behaviour in zebrafish (Danio rerio). Behav. Brain Res. 184, 157-166. https://doi.org/10.1016/j.bbr.2007.07.007

Mishara, A., Bonoldi, I., Allen, P., Rutigliano, G., Perez, J., Fusar-Poli, P., McGuire, P., 2016. Neurobiological models of self-disorders in early schizophrenia. Schizophr. Bull. 42, 874-880. https://doi.org/10.1093/schbul/sbv123.

Morris, J.A., 2009. Chapter 11 - zebrafish: a model system to examine the neurodevelopmental basis of schizophrenia. In: Akira, S. (Ed.), Progress in Brain Research, Genetic Models of Schizophrenia. Elsevier, pp. 97-106. https://doi.org/10.1016/ S0079-6123(09)17911-6.

Mueller, D.T., Wullimann, M., 2015. Atlas of Early Zebrafish Brain Development: A Tool for Molecular Neurogenetics. Academic Press.

Murray, R.M., Paparelli, A., Morrison, P.D., Marconi, A., Di Forti, M., 2013. What can we learn about schizophrenia from studying the human model, drug-induced psychosis? Am. J. Med. Genet. B Neuropsychiatr. Genet. 162B, 661-670. https://doi.org/10. 1002/ajmg.b.32177.

Nakai, T., Nagai, T., Wang, R., Yamada, S., Kuroda, K., Kaibuchi, K., Yamada, K., 2014. Alterations of GABAergic and dopaminergic systems in mutant mice with disruption of exons 2 and 3 of the Disc1 gene. Neurochem. Int. 74, 74-83. https://doi.org/10 1016/j.neuint.2014.06.009.

Nawaz, R., Asif, H., Khan, A., Ishtiaq, H., Shad, F., Siddiqui, S., 2014. Drugs targeting SNPrs35753505 of the NRG1 gene may prevent the association of neurological disorder schizophrenia in a Pakistani population. CNS Neurol. Disord. Drug Targets 13, 1604-1614.

Ng, M.-C., Hsu, C.-P., Wu, Y.-J., Wu, S.-Y., Yang, Y.-L., Lu, K.-T., 2012. Effect of MK-801 induced impairment of inhibitory avoidance learning in zebrafish via inactivation of extracellular signal-regulated kinase (ERK) in telencephalon. Fish Physiol. Biochem. 38, 1099-1106. https://doi.org/10.1007/s10695-011-9595-8.

O’Tuathaigh, C.M.P., Babovic, D., O'Sullivan, G.J., Clifford, J.J., Tighe, O., Croke, D.T., Harvey, R., Waddington, J.L., 2007. Phenotypic characterization of spatial cognition and social behavior in mice with "knockout" of the schizophrenia risk gene neuregulin 1. Neuroscience 147, 18-27. https://doi.org/10.1016/j.neuroscience.2007.03. eguli.

Pae, C.-U., Kim, J.-J., Serretti, A., Lee, C.-U., Lee, S.-J., Lee, C., Paik, I.-H., 2003. VNTR polymorphism of tyrosine hydroxylase gene and schizophrenia in the korean population. NPS 47, 131-136. https://doi.org/10.1159/000070581.

Paine, T.A., Carlezon, W.A., 2009. Effects of antipsychotic drugs on MK-801-induced attentional and motivational deficits in rats. Neuropharmacology 56, 788-797. https://doi.org/10.1016/j.neuropharm.2009.01.004.

Pal, P., Mihanović, M., Molnar, S., Xi, H., Sun, G., Guha, S., Jeran, N., Tomljenović, A., Malnar, A., Missoni, S., Deka, R., Rudan, P., 2009. Association of tagging single nucleotide polymorphisms on 8 candidate genes in dopaminergic pathway with schizophrenia in Croatian population. Croat. Med. J. 50, 361-369. https://doi.org/ schizophrenia in Croatian

Panula, P., Chen, Y.-C., Priyadarshini, M., Kudo, H., Semenova, S., Sundvik, M., Sallinen, V., 2010. The comparative neuroanatomy and neurochemistry of zebrafish CNS systems of relevance to human neuropsychiatric diseases. Neurobiol. Dis. 40, 46-57. https://doi.org/10.1016/j.nbd.2010.05.010.

Patel, K.R., Cherian, J., Gohil, K., Atkinson, D., 2014. Schizophrenia: Overview And Treatment Options. P T 39. pp. 638-645.

Purcell, S.M., Moran, J.L., Fromer, M., Ruderfer, D., Solovieff, N., Roussos, P., O'Dushlaine, C., Chambert, K., Bergen, S.E., Kähler, A., Duncan, L., Stahl, E., Genovese, G., Fernández, E., Collins, M.O., Komiyama, N.H., Choudhary, J.S. Magnusson, P.K.E., Banks, E., Shakir, K., Garimella, K., Fennell, T., DePristo, M., Grant, S.G.N., Haggarty, S.J., Gabriel, S., Scolnick, E.M., Lander, E.S., Hultman, C.M., Sullivan, P.F., McCarroll, S.A., Sklar, P., 2014. A polygenic burden of rare disruptive mutations in schizophrenia. Nature 506, 185-190. https://doi.org/10.1038/ nature12975.

Randlett, O., Wee, C.L., Naumann, E.A., Nnaemeka, O., Schoppik, D., Fitzgerald, J.E., Portugues, R., Lacoste, A.M.B., Riegler, C., Engert, F., Schier, A.F., 2015. Whole-brain activity mapping onto a zebrafish brain atlas. Nat. Methods 12, 1039-1046. https:// activity mapping onto a zebra doi.org $10.1038 /$ nmeth.3581.

Ratta-Apha, W., Mouri, K., Boku, S., Ishiguro, H., Okazaki, S., Otsuka, I., Sora, I., Arinami, T., Shirakawa, O., Hishimoto, A., 2015. A decrease in protein level and a missense polymorphism of KIF17 are associated with schizophrenia. Psychiatry Res. 230, 424-429. https://doi.org/10.1016/j.psychres.2015.09.031.

Riehl, R., Kyzar, E., Allain, A., Green, J., Hook, M., Monnig, L., Rhymes, K., Roth, A. Pham, M., Razavi, R., DiLeo, J., Gaikwad, S., Hart, P., Kalueff, A.V., 2011. Behaviora and physiological effects of acute ketamine exposure in adult zebrafish. Neurotoxicol. Teratol. 33, 658-667. https://doi.org/10.1016/j.ntt.2011.05.011.

Rihel, J., Prober, D.A., Arvanites, A., Lam, K., Zimmerman, S., Jang, S., Haggarty, S.J., 
Kokel, D., Rubin, L.L., Peterson, R.T., Schier, A.F., 2010. Zebrafish behavioral profiling links drugs to biological targets and rest/wake regulation. Science 327, 348-351. https://doi.org/10.1126/science.1183090.

Rinkwitz, S., Mourrain, P., Becker, T.S., 2011. Zebrafish: an integrative system for neurogenomics and neurosciences. Prog. Neurobiol. 93, 231-243. https://doi.org/10. 1016/j.pneurobio.2010.11.003.

Saleem, S., Shaukat, F., Gul, A., Arooj, M., Malik, A., 2017. Potential role of amino acids in pathogenesis of schizophrenia. Int J Health Sci (Qassim) 11, 63-68.

Savio, L.E.B., Vuaden, F.C., Piato, A.L., Bonan, C.D., Wyse, A.T.S., 2012. Behaviora changes induced by long-term proline exposure are reversed by antipsychotics in zebrafish. Prog. Neuropsychopharmacol. Biol. Psychiatry 36, 258-263. https://doi. org/10.1016/j.pnpbp.2011.10.002.

Schizophrenia Working Group of the Psychiatric Genomics Consortium, 2014. Biological insights from 108 schizophrenia-associated genetic loci. Nature 511, 421-427. https://doi.org/10.1038/nature13595.

Seibt, K.J., Oliveira, R., da, L., Zimmermann, F.F., Capiotti, K.M., Bogo, M.R., Ghisleni, G., Bonan, C.D., 2010. Antipsychotic drugs prevent the motor hyperactivity induced by psychotomimetic MK-801 in zebrafish (Danio rerio). Behav. Brain Res. 214, 417-422. https://doi.org/10.1016/j.bbr.2010.06.014

Selvaraj, S., Arnone, D., Cappai, A., Howes, O., 2014. Alterations in the serotonin system in schizophrenia: a systematic review and meta-analysis of postmortem and molecular imaging studies. Neurosci. Biobehav. Rev. 45, 233-245. https://doi.org/10. 1016/j.neubiorev.2014.06.005.

Sertori, R., Trengove, M., Basheer, F., Ward, A.C., Liongue, C., 2016. Genome editing in zebrafish: a practical overview. Brief. Funct. Genomics 15, 322-330. https://doi.org/ 10.1093/bfgp/elv051.

Singh, K.K., De Rienzo, G., Drane, L., Mao, Y., Flood, Z., Madison, J., Ferreira, M., Bergen, S., King, C., Sklar, P., Sive, H., Tsai, L.-H., 2011. Common DISC1 polymorphisms disrupt Wnt/GSK3 3 signaling and brain development. Neuron 72, 545-558. https:// doi.org/10.1016/j.neuron.2011.09.030.

Sison, M., Gerlai, R., 2011a. Associative learning performance is impaired in zebrafish (Danio rerio) by the NMDA-R antagonist MK-801. Neurobiol. Learn. Mem. 96, 230-237. https://doi.org/10.1016/j.nlm.2011.04.016.

Sison, M., Gerlai, R., 2011b. Behavioral performance altering effects of MK-801 in zebrafish (Danio rerio). Behav. Brain Res. 220, 331-337. https://doi.org/10.1016/j.bbr. 2011.02.019.

Stefansson, H., Sigurdsson, E., Steinthorsdottir, V., Bjornsdottir, S., Sigmundsson, T., Ghosh, S., Brynjolfsson, J., Gunnarsdottir, S., Ivarsson, O., Chou, T.T., Hjaltason, O., Birgisdottir, B., Jonsson, H., Gudnadottir, V.G., Gudmundsdottir, E., Bjornsson, A., Ingvarsson, B., Ingason, A., Sigfusson, S., Hardardottir, H., Harvey, R.P., Lai, D., Zhou, M., Brunner, D., Mutel, V., Gonzalo, A., Lemke, G., Sainz, J., Johannesson, G., Andresson, T., Gudbjartsson, D., Manolescu, A., Frigge, M.L., Gurney, M.E., Kong, A. Gulcher, J.R., Petursson, H., Stefansson, K. 2002. Neuregulin 1 and susceptibility to Gulcher, J.R., Petursson, H., Stefansson, K., 2002. Neuregulin 1 and susceptibility to

Suster, M.L., Sumiyama, K., Kawakami, K., 2009. Transposon-mediated BAC transgenesis in zebrafish and mice. BMC Genomics 10, 477. https://doi.org/10.1186/1471-2164. $10-477$

Swain, H.A., Sigstad, C., Scalzo, F.M., 2004. Effects of dizocilpine (MK-801) on circling behavior, swimming activity, and place preference in zebrafish (Danio rerio). Neurotoxicol. Teratol. 26, 725-729. https://doi.org/10.1016/j.ntt.2004.06.009.

Szatkiewicz, J.P., O’Dushlaine, C., Chen, G., Chambert, K., Moran, J.L., Neale, B.M., Fromer, M., Ruderfer, D., Akterin, S., Bergen, S.E., Kähler, A., Magnusson, P.K.E., Kim, Y., Crowley, J.J., Rees, E., Kirov, G., O’Donovan, M.C., Owen, M.J., Walters, J., Scolnick, E., Sklar, P., Purcell, S., Hultman, C.M., McCarroll, S.A., Sullivan, P.F., 2014. Copy number variation in schizophrenia in Sweden. Mol. Psychiatry 19, 762-773. https://doi.org/10.1038/mp.2014.40.

Szeszko, P.R., Hodgkinson, C.A., Robinson, D.G., Derosse, P., Bilder, R.M., Lencz, T., Burdick, K.E., Napolitano, B., Betensky, J.D., Kane, J.M., Goldman, D., Malhotra, A.K., 2008. DISC1 is associated with prefrontal cortical gray matter and positive symptoms in schizophrenia. Biol. Psychol. 79, 103-110. https://doi.org/10.1016/j. biopsycho.2007.10.011.

Tachikawa, H., Harada, S., Kawanishi, Y., Okubo, T., Suzuki, T., 2001. Polymorphism of the 5 -Upstream region of the human SNAP-25 gene:an association analysis with schizophrenia. NPS 43, 131-133. https://doi.org/10.1159/000054880.

Takahashi, M., Inoue, M., Tanimoto, M., Kohashi, T., Oda, Y., 2017. Short-term desensitization of fast escape behavior associated with suppression of Mauthner cell activity in larval zebrafish. Neurosci. Res. 121, 29-36. https://doi.org/10.1016/j. neures.2017.03.008.

Takahashi, M., Narushima, M., Oda, Y., 2002. In vivo imaging of functional inhibitory networks on the mauthner cell of larval zebrafish. J. Neurosci. 22, 3929-3938. https://doi.org/10.1523/JNEUROSCI.22-10-03929.2002.

Tarabeux, J., Champagne, N., Brustein, E., Hamdan, F.F., Gauthier, J., Lapointe, M., Maios, C., Piton, A., Spiegelman, D., Henrion, E., Synapse to Disease Team, Millet, B., Rapoport, J.L., Delisi, L.E., Joober, R., Fathalli, F., Fombonne, E., Mottron, L., ForgetDubois, N., Boivin, M., Michaud, J.L., Lafrenière, R.G., Drapeau, P., Krebs, M.-O., Rouleau, G.A., 2010. De novo truncating mutation in Kinesin 17 associated with schizophrenia. Biol. Psychiatry 68, 649-656. https://doi.org/10.1016/j.biopsych. 2010.04.018.
Thyme, S.B., Pieper, L.M., Li, E.H., Pandey, S., Wang, Y., Morris, N.S., Sha, C., Choi, J.W., Herrera, K.J., Soucy, E.R., Zimmerman, S., Randlett, O., Greenwood, J., McCarroll, S.A., Schier, A.F., 2019. Phenotypic landscape of schizophrenia-associated genes defines candidates and their shared functions. Cell 177, 478-491. https://doi.org/10. 1016/j.cell.2019.01.048. e20.

Tomoda, T., Sumitomo, A., Jaaro-Peled, H., Sawa, A., 2016. Utility and validity of DISC1 mouse models in biological psychiatry. Neuroscience 321, 99-107. https://doi.org/ 10.1016/j.neuroscience.2015.12.061.

Tran, S., Muraleetharan, A., Fulcher, N., Chatterjee, D., Gerlai, R., 2016. MK-801 increases locomotor activity in a context-dependent manner in zebrafish. Behav. Brain Res. 296, 26-29. https://doi.org/10.1016/j.bbr.2015.08.029.

Tremolizzo, L., Doueiri, M.-S., Dong, E., Grayson, D.R., Davis, J., Pinna, G., Tueting, P., Rodriguez-Menendez, V., Costa, E., Guidotti, A., 2005. Valproate corrects the schizophrenia-like epigenetic behavioral modifications induced by methionine in mice. zophrenia-like epigenetic behavioral modifications induced by methionine in mice.
Biol. Psychiatry 57, 500-509. https://doi.org/10.1016/j.biopsych.2004.11.046.

Wang, L., Alachkar, A., Sanathara, N., Belluzzi, J.D., Wang, Z., Civelli, O., 2015. A methionine-induced animal model of schizophrenia: face and predictive validity. Int. J. Neuropsychopharmacol. 18. https://doi.org/10.1093/ijnp/pyv054

Wang, L., Jiang, W., Lin, Q., Zhang, Y., Zhao, C., 2016. DNA methylation regulates gabrb2 mRNA expression: developmental variations and disruptions in 1-methionine-induce zebrafish with schizophrenia-like symptoms. Genes Brain Behav. 15, 702-710. https://doi.org/10.1111/gbb.12315.

Washbourne, P., Thompson, P.M., Carta, M., Costa, E.T., Mathews, J.R., Lopez-Benditó, G., Molnár, Z., Becher, M.W., Valenzuela, C.F., Partridge, L.D., Wilson, M.C., 2002. Genetic ablation of the t-SNARE SNAP-25 distinguishes mechanisms of neuroexocytosis. Nat. Neurosci. 5, 19-26. https://doi.org/10.1038/nn783.

Winchester, C.L., Pratt, J.A., Morris, B.J., 2014. Risk genes for schizophrenia: translational opportunities for drug discovery. Pharmacol. Ther. 143, 34-50. https://doi. org $/ 10.1016 /$ j.pharmthera.2014.02.003.

Wood, J.D., Bonath, F., Kumar, S., Ross, C.A., Cunliffe, V.T., 2009. Disrupted-in-schizophrenia 1 and neuregulin 1 are required for the specification of oligodendrocytes and neurones in the zebrafish brain. Hum. Mol. Genet. 18, 391-404. https://doi.org/10. 1093/hmg/ddn361.

Wullimann, M.F., Mueller, T., 2004. Teleostean and mammalian forebrains contrasted: evidence from genes to behavior. J. Comp. Neurol. 475, 143-162. https://doi.org/10 1002/cne.20183.

Wullimann, M.F., Rupp, B., Reichert, H., 1996a. Functional anatomy of the zebrafish brain: a comparative evaluation. In: Wullimann, M.F., Rupp, B., Reichert, H. (Eds.), Neuroanatomy of the Zebrafish Brain: A Topological Atlas. Birkhäuser Basel, Basel, pp. 89-101. https://doi.org/10.1007/978-3-0348-8979-7_6.

Wullimann, M.F., Rupp, B., Reichert, H., 1996b. Introduction: neuroanatomy for a neurogenetic model system. In: Wullimann, M.F., Rupp, B., Reichert, H. (Eds.), Neuroanatomy of the Zebrafish Brain: A Topological Atlas. Birkhäuser Basel, Basel, pp. 1-5. https://doi.org/10.1007/978-3-0348-8979-7_1.

Wullimann, M.F., Rupp, B., Reichert, H., 1996c. Taxonomic background. In: Wullimann, M.F., Rupp, B., Reichert, H. (Eds.), Neuroanatomy of the Zebrafish Brain: A Topological Atlas. Birkhäuser Basel, Basel, https://doi.org/10.1007/978-3-03488979-7_2. pp. 5-5.

Wullimann, M.F., Rupp, B., Reichert, H., 1996d. Technical details. In: Wullimann, M.F., Rupp, B., Reichert, H. (Eds.), Neuroanatomy of the Zebrafish Brain: A Topological Atlas. Birkhäuser Basel, Basel, https://doi.org/10.1007/978-3-0348-8979-7_3. pp. 6-6.

Wullimann, M.F., Rupp, B., Reichert, H., 1996e. The brain of the zebrafish Danio rerio: neuroanatomical atlas. In: Wullimann, M.F., Rupp, B., Reichert, H. (Eds.), Neuroanatomy of the Zebrafish Brain: A Topological Atlas. Birkhäuser Basel, Basel, pp. 19-87. https://doi.org/10.1007/978-3-0348-8979-7_5.

Wullimann, M.F., Rupp, B., Reichert, H., 1996f. The brain of the zebrafish Danio rerio: an overview. In: Wullimann, M.F., Rupp, B., Reichert, H. (Eds.), Neuroanatomy of the Zebrafish Brain: A Topological Atlas. Birkhäuser Basel, Basel, pp. 7-17. https://doi. org/10.1007/978-3-0348-8979-7 4.

Wyatt, C., Bartoszek, E.M., Yaksi, E., 2015. Methods for studying the zebrafish brain: past, present and future. Eur. J. Neurosci. 42, 1746-1763. https://doi.org/10.1111/ejn. 12932

Xu, X., Scott-Scheiern, T., Kempker, L., Simons, K., 2007. Active avoidance conditioning in zebrafish (Danio rerio). Neurobiol. Learn. Mem. 87, 72-77. https://doi.org/10. 1016/j.nlm.2006.06.002.

Zakhary, S.M., Ayubcha, D., Ansari, F., Kamran, K., Karim, M., Leheste, J.R., Horowitz, J.M., Torres, G., 2011. A behavioral and molecular analysis of ketamine in zebrafish. Synapse 65, 160-167. https://doi.org/10.1002/syn.20830.

Zhou, Yang, Kaiser, T., Monteiro, P., Zhang, X., Van der Goes, M.S., Wang, D., Barak, B. Zeng, M., Li, C., Lu, C., Wells, M., Amaya, A., Nguyen, S., Lewis, M., Sanjana, N., Zhou, Yongdi, Zhang, M., Zhang, F., Fu, Z., Feng, G., 2016. Mice with Shank3 muZhou, Yongdi, Zhang, M., Zhang, F., Fu, Z., Feng, G., 2016. Mice with Shank3 mu-
tations associated with ASD and schizophrenia display both shared and distinct detations associated with ASD and schizophrenia display both shared and distin.
fects. Neuron 89, 147-162. https://doi.org/10.1016/j.neuron.2015.11.023.

Zimmermann, F.F., Gaspary, K.V., Siebel, A.M., Bonan, C.D., 2016. Oxytocin reversed MK-801-induced social interaction and aggression deficits in zebrafish. Behav. Brain Res. 311, 368-374. https://doi.org/10.1016/j.bbr.2016.05.059. 

Paper II

\section{Pharmacological validation of the prepulse inhibition of startle response in larval zebrafish using a commercial automated system and software}

Banono, Nancy Saana \& Esguerra, Camila V.

Published in Journal of Visualized Experiments, April 2020, volume 161, DOI: $10.3791 / 61423$ 



\title{
Pharmacological Validation of the Prepulse Inhibition of Startle Response in Larval Zebrafish using a Commercial Automated System and Software
}

\author{
Nancy Saana Banono ${ }^{1,2}$, Camila V. Esguerra ${ }^{1,2}$ \\ ${ }^{1}$ Chemical Neuroscience Group, Centre for Molecular Medicine Norway (NCMM), Faculty of Medicine, University of Oslo ${ }^{2}$ Section for Pharmacology and \\ Pharmaceutical Biosciences, Department of Pharmacy, Faculty of Mathematics and Natural Sciences, University of Oslo
}

\section{Corresponding Author}

Camila V. Esguerra

c.v.esguerra@ncmm.uio.no

\section{Citation}

Banono, N.S.

Esguerra, C.V. Pharmacological

Validation of the Prepulse Inhibition of Startle Response in Larval Zebrafish using a Commercial Automated System and Software. J. Vis. Exp. (161), e61423, doi:10.3791/61423 (2020).

\section{Date Published}

July 2, 2020

DOI

$10.3791 / 61423$

URL

jove.com/video/61423

\section{Abstract}

While there is an abundance of commercial and standardized automated systems and software for performing the prepulse inhibition (PPI) assay in rodents, to the best of our knowledge, all PPI assays performed in the zebrafish have, until now, been done using custom made systems which were only available to individual groups. This has thereby presented challenges, particularly with regard to issues of data reproducibility and standardization. In the present work, we generated a protocol that utilizes commercially available automated systems to pharmacologically validate the PPI assay in larval zebrafish. Consistent with published findings, we were able to replicate the results of apomorphine, haloperidol and ketamine on the PPI response of 6 days post-fertilization zebrafish larvae.

\section{Introduction}

The zebrafish (Danio rerio) larva is a suitable candidate for modelling psychiatric diseases such as schizophrenia (reviewed by Gawel et al. $^{1}$ ) because of the numerous advantages it possesses. These include a fully sequenced genome with $70 \%$ sequence homology to human orthologues $^{2}$, existence of forward and reverse genetic tools to manipulate the genome and to identify the contribution of a given gene towards development or disease ${ }^{3}$, and the presence of major human/rodent neurotransmitters in the zebrafish brain ${ }^{4}$. There is an availability of several neuro-phenotypic domains in zebrafish, such as anxiety, learning and memory ${ }^{3}$. Optical transparency and sensitivity to the major classes of neurotropic drugs makes it an ideal 
candidate of choice for pharmacological manipulations and phenotypic drug screening ${ }^{5,6}$.

To perform high throughput drug screening, automation and the presence of a robust endophenotype is highly important $^{7}$. For instance, a variety of automatic recording techniques have been developed for measuring larval zebrafish behavior such as thigmotaxis, startle response, optokinetic response, optomotor response, habituation, prey capture, sleep/wake behavior, locomotor behavior and several others ${ }^{6}$. While some laboratories develop custombuilt systems for automated measurements and analysis of some of these behaviors, there are commercially available imaging and software systems ${ }^{8}, 9,10,11$. Prepulse inhibition (PPI), a form of sensorimotor gating in which the startle response is reduced when a weak non-startling stimulus is presented briefly before the startling stimulus, has been used as an endophenotype for studying schizophrenia in animal models (reviewed by ${ }^{12,13}$ ). In addition, acoustic startle response (ASR) and PPI have played useful roles in studying hearing and auditory function in animal models including the zebrafish $^{14,15}$. The larval zebrafish displays a characteristic C-start in response to an unexpected startling stimulus that is lessened by a weaker stimulus called the prepulse. The C-start has long been described as an escape behavior controlled by distinct neural cell populations and has been thoroughly characterized in the larval zebrafish ${ }^{15}, 16,17$.

There is an abundance of commercial and standardized automated systems and software for performing the PPI assay in rodents ${ }^{18,19,20}$. However, to the best of our knowledge, all the PPI assays performed in the zebrafish until now have been done using custom made systems which are only available to the individual groups ${ }^{15,16,21,22}$. This presents challenges for achieving data reproducibility and replicability with regard to standardization ${ }^{23}$.

Recently, a known vendor in the zebrafish community developed a set-up embedded with a fast camera and PPI generator add-ons to carry out the PPI assay in larval zebrafish $^{24}$. The camera records at 1000 frames per second which enables the recording of fast acting behaviors such as the C-start, while the PPI generator allows for user-controlled delivery of various acoustic stimuli to evoke a startle response ${ }^{24}$. Here, we combine the aforementioned system with a commercially available comprehensive software package designed for the automated analysis of complex behaviors $^{11}$, to generate a protocol for performing PPI response assays in larval zebrafish. We pharmacologically validate the PPI response using 1) apomorphine, a dopamine agonist known to cause deficits in PPI; 2) haloperidol, a dopamine antagonist and antipsychotic known to enhance PPI and 3) ketamine, a NMDA receptor antagonist known to modulate PPI.

\section{Protocol}

All animal experiments were approved by the Norwegian Food Safety Authority experimental animal administration's supervisory and application system (FOTS-18/106800-1).

\section{Zebrafish husbandry}

1. Set up matings of wild type adult male and female zebrafish (Danio rerio) stocks, maintained under standard conditions $^{25}$ the evening before. Here, Tupfel long-fin $(T L)$ strain is used.

2. Remove barriers the next morning and allow to mate through natural spawning.

3. Collect eggs out of the mating tanks. 
4. Remove unfertilized eggs and other debris, then transfer eggs to petri dishes $(n=60)$ and raise in an incubator at $28^{\circ} \mathrm{C}$ in embryo medium: $1.5 \mathrm{mM}$ HEPES, $\mathrm{pH} 7.6,17.4$ $\mathrm{mM} \mathrm{NaCl}, 0.21 \mathrm{mM} \mathrm{KCl}, 0.12 \mathrm{mM} \mathrm{MgSO}$, and $0.18 \mathrm{mM}$ $\mathrm{Ca}(\mathrm{NO} 3)_{2}$

5. Renew half of the embryo medium and remove dead larvae daily until $6 \mathrm{dpf}$.

NOTE: All experiments were performed on individual larvae at 6 days post-fertilization (dpf).

\section{Pharmacological agents and pre-treatment of larvae}

1. Dissolve apomorphine and ketamine in E3 medium to make $500 \mu \mathrm{M}$ and $10 \mathrm{mM}$ stock solutions respectively.

2. Dissolve haloperidol in $100 \%$ dimethyl sulfoxide (DMSO) to make a $10 \mathrm{mM}$ stock solution. The final concentration of DMSO used was $0.1 \%$.

3. Use $0.1 \%$ DMSO and E3 medium as vehicle controls.

4. Use the following final concentrations of drugs: 10 $\mathrm{mg} / \mathrm{mL}$ of apomorphine, $1 \mathrm{mM}$ ketamine and $20 \mu \mathrm{M}$ haloperidol $^{16}$.

5. Pre-expose the apomorphine and ketamine groups larvae for $10 \mathrm{~min}$ and the haloperidol and DMSO vehicle control groups for $20 \min ^{16}$.

\section{Setup prior to the behavior test}

1. On the day of the experiment, transfer larvae and all relevant materials into the experiment room. Set the experiment room to a temperature of $27 \pm 1{ }^{\circ} \mathrm{C}$.

2. Ensure that the background noise in the test chamber is as low as possible, preferably not more than $45 \mathrm{~dB}$ sound pressure level (SPL).
1. Install the sonometer microphone of the decibel (dB) meter in the test chamber (the opening for installation is already bored by the manufacturer).

2. To reduce the background noise in the room, insulate the test chamber with a custom-built sound booth (see Figure 1B for an overview of the set-up).

3. Prepare a 96-well plate for the prepulse inhibition test. NOTE: The video camera has a $2048 \times 500$ pixel resolution, meaning only a maximum of 3 lanes (33 wells) can be imaged at a time.

1. Use a custom-made acrylic plate of 96-well format to reduce interference from shadows.

NOTE: The measurements for the custom plate can be found at the following website: https://zenodo.org/ record/3739378\#.XooyLW5uKas

4. With the aid of a transfer pipette, transfer $310 \mu \mathrm{L}$ of exposure solution/medium with one larva into each well.

5. Calibrate and measure the stimulus intensity using the volume knob of the stereo amplifier and a decibel meter respectively.

6. Register the maximum sound intensity in the "level reference" section.

\section{Stimulus parameters and video acquisition}

1. Turn on the computer, the amplifier system and the dB meter (see Figure 1A for an overview of set-up).

2. Use the volume knob by turning it to minimum or maximum to adjust the sound intensity.

1. Check the sound level with the $\mathrm{dB}$ meter each time, the volume knob is adjusted. This is important in finding the maximum and minimum sound intensity that can be produced by the set-up. 
NOTE: The dB meter computes the RMS dB output for the stimulus. The system generates the sound inside the solid components of the test-chamber, keeping the plate firm while producing a vibration in the horizontal plane of the entire plate support.

1. Adjust the volume knob to maximum, measure the sound intensity with the $\mathrm{dB}$ meter and use this value.

3. On the interface of the PPI generator, define the parameters: inter-stimulus interval represented as Delay; inter-trial interval represented as Acquisition delta time; duration of prepulse etc.

1. For prepulse alone trials, ensure that the "Amplitude" or Duration of stimulus for Startle parameters are set to zero and vice versa for startle alone trials.

4. To generate a trial list, select Add > give a name to the trial. For example, "Prepulse $50 \mathrm{~dB}$ alone".

NOTE: One can generate as many trials as desired, but be careful of how long the list is since this can crash the program.

1. Interleave prepulse trials with pulse alone trials in all PPI experiments using a pseudorandom order. Where multiple stimuli are presented in an experiment, an inter-trial interval (ITI) of $30 \mathrm{~s}$ is used.

NOTE: In this study, a 100 ms startle stimulus (pulse) of $660 \mathrm{~Hz}$, and $5 \mathrm{~ms}$ prepulse stimuli of $440 \mathrm{~Hz}$ were used. For PPI experiments, inter-stimulus interval (ISI) was $100 \mathrm{~ms}$.

5. To save the protocol, select File > Save as.

6. Adjust lighting conditions in the test-chamber as follows.

1. Launch USB measurement computing, select analog out then go to D/A OUT O (P13) to make changes to the lighting. A value of zero means no light while increasing the D/A OUT O value, increases the intensity of light in the box. Light intensity of 100 was used for all experiments.

7. Set-up the camera

1. Launch the software and wait for the camera to load.

2. Select Adjustments (found on right-hand side) and set the acquisition frame rate to 1,000 then click apply to effect the change.

8. Acclimate larvae to a $100 \%$ light illuminated test chamber for $5 \mathrm{~min}$ before the experiments are started.

9. To begin an experiment, select the Experiment menu on the PPI generator, click Run and the select well format (e.g., 33 wells).

1. Always make sure that the camera software is launched with the right settings before running an experiment.

2. Acquire a $2 \mathrm{~s}$ video for each trial.

3. Make sure that the acquisition frame rate is set at 1,000 .

\section{Automated tracking and analysis of acoustic startle response and PPI}

1. Protocol setup.

1. Launch the analysis software (see the Table of Materials). Choose New from template > Apply a predefined template and then go through other menus (details below).

1. Choose from video file under Video Source.

2. Browse video file. Set subject as fish $>$ zebrafish larvae > zone template (no template). Specify Number of arenas under Arenas. 
3. Specify Number of subjects per arena (set as 1) under Subjects.

4. Select Center-point, nose-point and tail-base detection under Tracked Features (see Figure 2A,B).

NOTE: This is important to calculate the body angle of the C-start response (see Figure $\mathbf{2 C}$ ).

5. Click Name $>$ save as. Units used are $\mathrm{mm}, \mathrm{s}$, deg for distance, time and rotation respectively.

NOTE: Remember to use the same unit for calibrating the scale.

\section{Choose Arena setting.}

1. Click Grab background image.

2. Go through the steps on the right-hand menu (if in doubt, use the Help menu).

3. Choose the circle drawing tool to draw the arenas.

3. Choose Trial control settings $>$ create new $>$ name.

4. Choose Detection settings, go through the steps on the right-hand menu.

1. Set sample rate to 25 . Choose advanced detection settings. Under Method, select dynamic subtraction, advanced model/adult fish, then set Subject color compared to background as Darker and move the slider to define the larva's contrast.

2. Under subject contour, select erode first, then dilate and increase the contour erosion and dilation values until the animal is completely detected.

5. Save the protocol and use for subsequent analyses of PPI videos acquired.
2. Trial list setup.

1. Choose trial list, define independent variables such as larval ID, treatment, stimulus type, etc. Select the path of videos and define a list of trials for batch acquisition.

3. Acquisition setup.

NOTE: If a trial list has been generated, one can perform a batch acquisition of the videos.

1. If some tracks are lost, use the track editor to adjust the tracked features.

2. Exclude from analysis, the tracking errors that remain unresolved after using the track editor.

3. Set track smoothing profile to $1 \mathrm{~mm}$ to decrease noise from data. This can be adjusted based on the background activity of larvae.

4. Analysis setup.

1. To select trials to be analyzed, choose Data profiles and define tracks based on the independent variable of interest.

NOTE: If components are hidden, click on the eye symbol to the upper right-hand corner to display.

1. Filter parts of trials to be analyzed (e.g., based on treatment or type of stimulus group).

2. Select part of the tracks to be analyzed (nesting). For this study, data was nested for tracks between onset of stimulus and $100 \mathrm{~ms}$ after stimulus onset.

3. Remember to connect all filters and nesting boxes with arrow lines to complete the instruction.

2. Define dependent variables to be analyzed, select Analysis profiles and specify the variables of interest (focus on Body under dependent variables). 
NOTE: If components are hidden, click on the eye symbol to the upper right-hand corner to display.

1. Double click Body angle. Select absolute bend. Go through Trial settings and select maximum, then click on add.

3. Double click body angle state. Set averaging interval to 5 samples. Set bend angle threshold. To calculate statistics for bent, go through Trial statistics and select latency to first $>$ add. Repeat steps until varying thresholds are obtained (between $20-80^{\circ}$ was used) and name accordingly.

4. Generating statistics and charts.

1. Choose analysis $>$ results $>$ statistics $\&$ charts, then click calculate.

2. Make sure the data and analysis profiles are set to the right template since several templates can be made under each section.

5. Export trial and group statistics as spreadsheet files for processing and analysis.

\section{Data analysis}

1. Open the spreadsheet file containing the trial statistics.

2. Select the columns Body angle Maximum deg, Bent latency (of the various body angle thresholds).

3. Consider every change in body angle $\geq 30^{\circ}$ within a cutoff latency of $50 \mathrm{~ms}$ after stimulus onset as a positive Cstart response (i.e., a responder); those with $<30^{\circ}$ body angle are non-responders.

4. In a binary fashion, assign 1 to a responding larva and 0 to non-responding larvae for each plate.

1. Count the total number of responders and nonresponder larvae for each plate. Calculate the responders (\%) in each case calculated as (number of larvae responding/total number of larvae) $\times 100$. Exclude larvae that respond less than $30 \%$ to the startle stimulus from the analysis ${ }^{16}$.

NOTE: Any stimulus intensity capable of eliciting a Cstart response in equal to or more than $70 \%$ of the larvae is considered a suitable startle stimulus ${ }^{16}$.

5. Calculate \%PPI as $100 \times$ (percentage responding to startle stimulus - percentage responding to prepulse + startle sequence)/ (percentage responding to startle stimulus $)^{16}$.

\section{Statistical analysis}

1. Present data as the mean \pm standard deviation, S.D. (see the Table of Materials for statistical software).

2. Determine the effects of varying prepulse intensities on larval response using one-way ANOVA followed by a Tukey's post-hoc test.

3. Use two-way ANOVA followed by Holm-Sidak's post-hoc test to determine the effects of drug treatment on \% PPI response with varying prepulse intensities.

\section{Representative Results}

Three experiments were performed to validate the protocol of combining multiple systems to analyze prepulse inhibition of acoustic startle response in the larval zebrafish. First, the ability to accurately deliver acoustic stimuli and to capture the response of larvae to the startle stimulus was tested. Next, was validating the ability to attenuate startle response when a prepulse stimulus is presented. Finally, the ability to detect the pharmacological modulation of prepulse inhibition of the startle response by the drugs apomorphine, haloperidol and ketamine was established. 


\section{Larval zebrafish response to acoustic startle stimuli}

Previous work has demonstrated that larval zebrafish display a characteristic C-start when presented with startling acoustic stimulus $^{16}$. The ability to incite and capture the behavior of larvae to the startle stimuli was tested. Recorded larvae were observed to display the C-start response (Figure 2). A stimulus of $70 \mathrm{~dB}$ re $(100 \mathrm{~ms}, 660 \mathrm{~Hz}$, Supplementary Figure 1A) was strong enough to elicit response in $\geq 70 \%$ of the larvae (Figure 3A). When repeatedly presented 30 times at an inter-trial interval of $30 \mathrm{~s}$, the $70 \mathrm{~dB}$ re stimulus did not result in larval habituation ( $\mathrm{N}=3$ replicates; 16 larvae/ replicate), as shown in Figure 3B.

\section{Prepulse decreases startle response of larval zebrafish} to acoustic stimuli

A plethora of evidence shows that prepulse stimuli modulates larval response to a startle stimulus ${ }^{15,21,22,26}$. A twopulse paradigm was used, where a weak stimulus called the prepulse preceded the startle-inducing stimulus called the pulse. The prepulse stimuli used were either 20, 17, or $14 \mathrm{~dB}$ less than the pulse stimulus that was set at 70 $\mathrm{dB}$ re. The prepulse $(5 \mathrm{~ms}, 440 \mathrm{~Hz})$ was always presented 100 ms before the pulse onset (Supplementary Figure 1B). Each tested prepulse stimulus significantly reduced larval response to the pulse. In Figure 4 the larval response (in \%) to acoustic startle stimuli is shown for $6 \mathrm{dpf} T L$ in E3 medium, $N=6$ (16 larvae/group). The percentage of larvae responding to the startle stimulus (pulse) was $79.86 \pm 9.772$. Expectedly, when the startle stimulus was preceded by either a 50,53 or $56 \mathrm{~dB}$ prepulse, the larval response decreased to $40.87 \% \pm 11.30 \%, 39.58 \% \pm 7.345 \%$ and $29.17 \% \pm$ 9.350\% respectively. One-way Anova analysis revealed a statistical difference in stimulus effect on larvae $(F(3,48)$ $=57.23, \mathrm{P}<0.0001)$ with Tukey's multiple comparisons test revealing statistical significance across groups at $95 \%$ confidence interval.

\section{Pharmacological modulation of prepulse inhibition}

Earlier studies showed that the dopaminergic drugs, apomorphine and haloperidol, as well as the glutamatergic drug, ketamine, significantly modulated prepulse inhibition in larvae just as in their mammal and rodent equivalents ${ }^{16}$. Concentrations for validation of the set-up were selected based on these studies. The inter-stimulus interval (ISI) for all the pharmacological experiments was $100 \mathrm{~ms}$.

\section{Effect of apomorphine on prepulse inhibition}

In Figure 5, larvae pretreated with $10 \mathrm{mg} / \mathrm{mL}$ apomorphine for 10 min displayed an overall reduction in \% PPI compared to E3 control larvae (two-way ANOVA, non RM (factors: treatment and prepulse intensities; treatment: $F(1,34)=$ 16.21, $p=0.0003$; prepulse intensity: $F(2,34)=8.674$, $P=0.0009$, this showed a non-significant interaction: $F(2$, $34)=2.514, p=0.0959)$. To investigate the differences in more detail, Holm-Sidak's post-hoc test revealed significant differences in the startle response between E3 control and apomorphine treated larvae at prepulse intensities $53(\mathrm{p}=$ $0.0126)$ and $56(p=0.0044)$ but not at $50 \mathrm{~dB}(p=0.5813)$.

\section{Effect of haloperidol on prepulse inhibition}

Figure 6 shows an overall increase in \%PPI in larvae pretreated for $20 \mathrm{~min}$ with $20 \mu \mathrm{M}$ haloperidol compared to those in E3 medium (two-way ANOVA, non RM (factors: treatment and prepulse intensities; treatment: $F(1,32)=$ 20.75, $p<0.0001$; prepulse intensity: $F(2,32)=3.147, p=$ 0.0565 , with no significant interaction: $F(2,32)=0.7455, p$ $=0.4826)$. Using the Holm-Sidak's post-hoc test, presence of statistical significance was observed only at prepulse 
intensities $53(p=0.00489$ and $56(p=0.0348)$ but not at 50 $d B(p=0.067)$.

\section{Effect of ketamine on prepulse inhibition}

Figure 7 shows that at different prepulse stimulus intensities, there were differences in the startle response between E3 control larvae and those pretreated for $10 \mathrm{~min}$ in $1.0 \mathrm{mM}$ ketamine (two-way ANOVA, non RM (factors: treatment and prepulse intensities; treatment: $F(1,35)=25.46, p<0.0001$; prepulse intensity: $F(2,35)=6.018, p=0.0057$, with no significant interaction: $F(2,35)=0.8450, p=0.4381)$. HolmSidak's post-hoc test, showed significance only at prepulse intensities of $50(p=0.0039)$ and $53(p=0.0027)$, but not at $56 \mathrm{~dB}(p=0.0802)$.

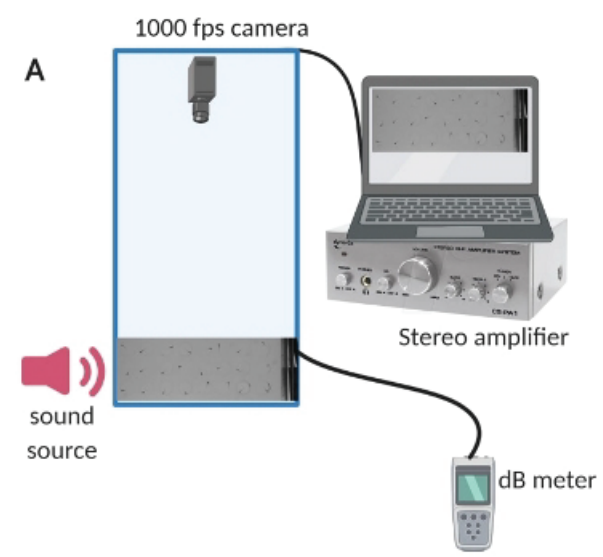

B

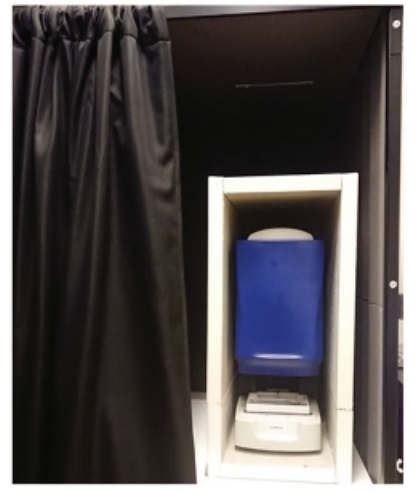

Figure 1: Testing apparatus. (A) Overview of equipment set-up. (B) In-house insulation of the set-up equipment to minimize background noise during experiments. Please click here to view a larger version of this figure. 

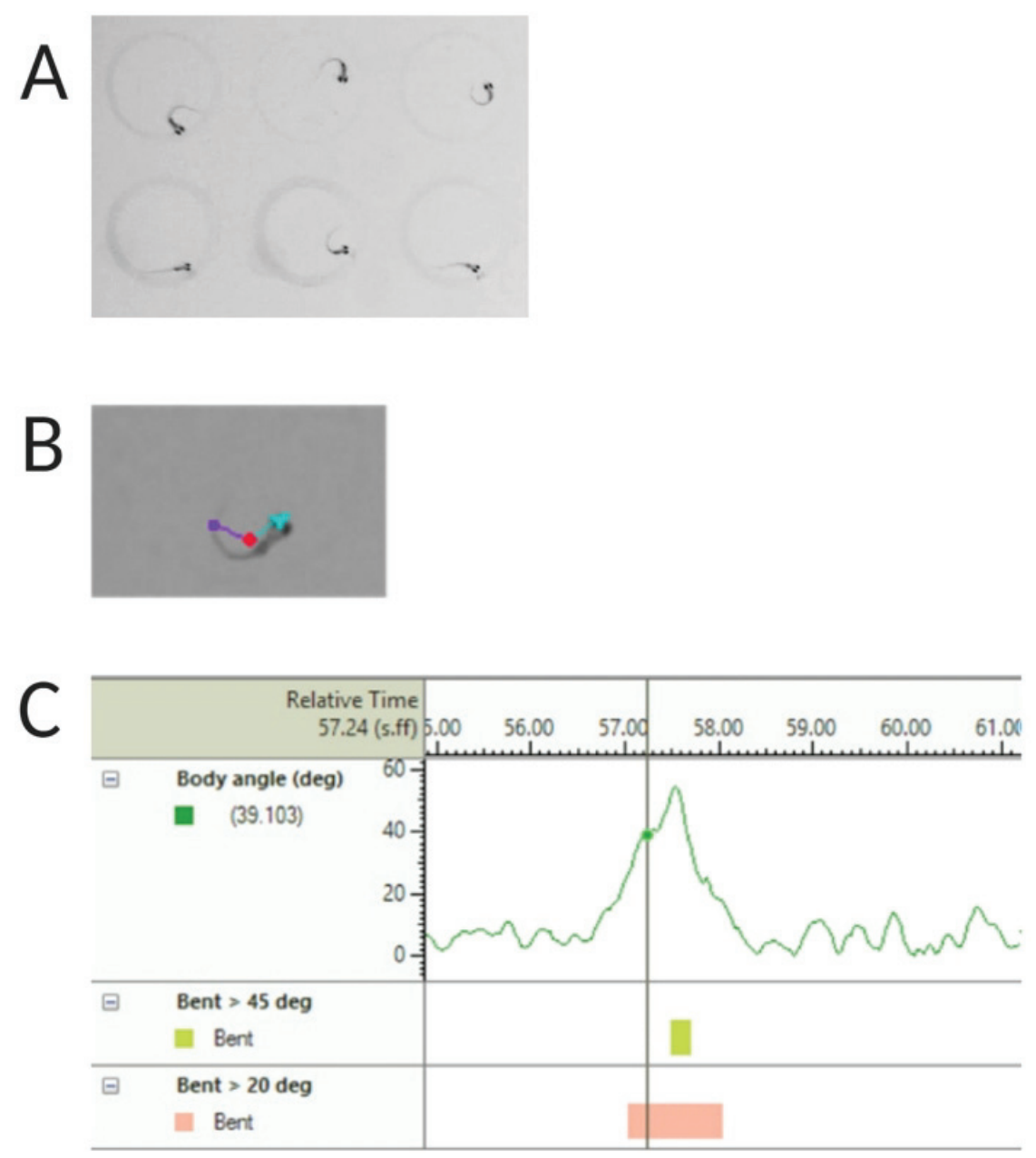

Figure 2: Analysis of the larval zebrafish acoustic startle response. (A) Characteristic C-start displayed by 6 zebrafish larvae at $6 \mathrm{dpf}$ (B) Representative image of the three tracked features superimposed on a $6 \mathrm{dpf}$ larva: center-point (red), nose-point (cyan) and tail-base (purple). (C) Representative image of the absolute bend angle displayed by a 6-dpf TL wild type larvae. Please click here to view a larger version of this figure. 

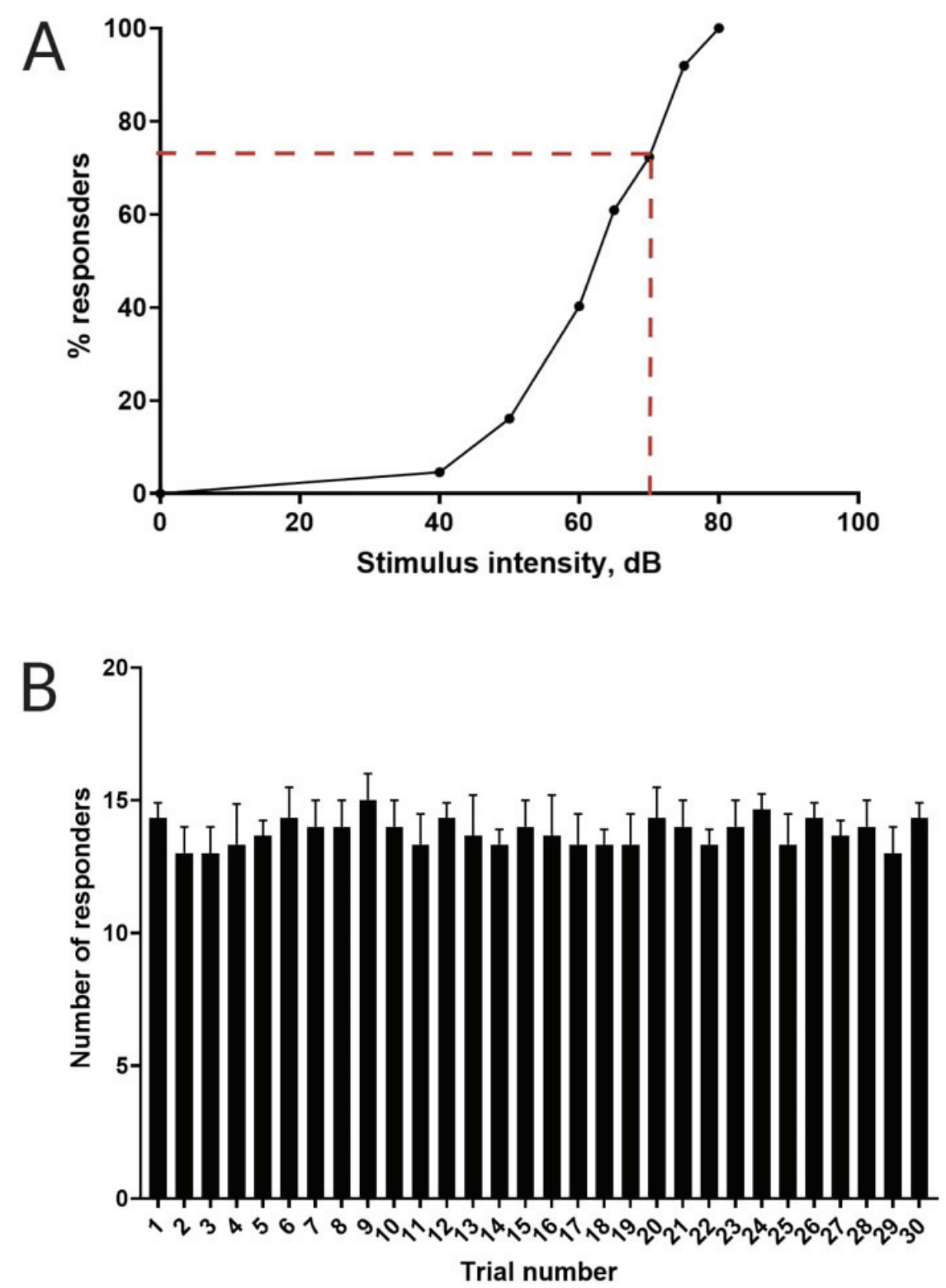

Figure 3: Determination of acoustic startle threshold. (A) A stimulus intensity of $70 \mathrm{~dB}$ (represented by red dash lines) is capable of eliciting a C-start response in $>70 \%$ of larvae $(\mathrm{N}=33 ; 6 \mathrm{dpf} \mathrm{TL})$. (B) Larvae do not habituate to $70 \mathrm{~dB}$ re stimulus presented 30 times (trials) at an inter-trial interval of $30 \mathrm{~s}(\mathrm{~N}=3$ replicates; 16 larvae/replicate). Data are presented as mean \pm S.D. Please click here to view a larger version of this figure. 


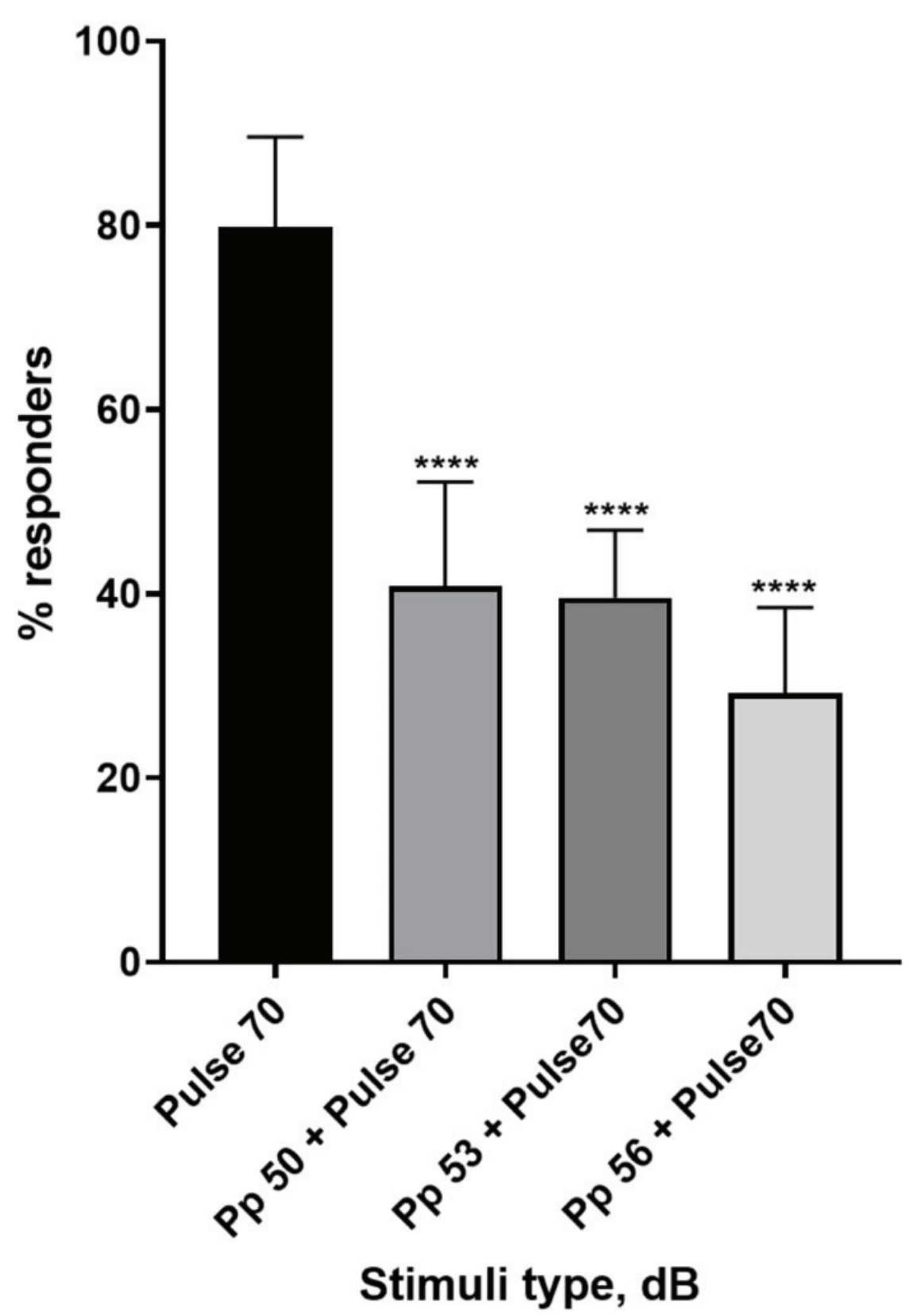

Figure 4: Pre-pulse-induced decrease in larval response (\%). Pre-pulse stimuli at 20, 17 and $14 \mathrm{~dB}$ lower than the $70 \mathrm{~dB}$ re startling stimulus cause a reduction in the number of wild type TL larvae C-start responders. All data represented as mean \pm S.D., $N=5$ (16 larvae/group), ${ }^{* * *} p<0.0001$, significantly different from startle stimulus by Tukey's post-hoc test after oneway ANOVA. Please click here to view a larger version of this figure. 


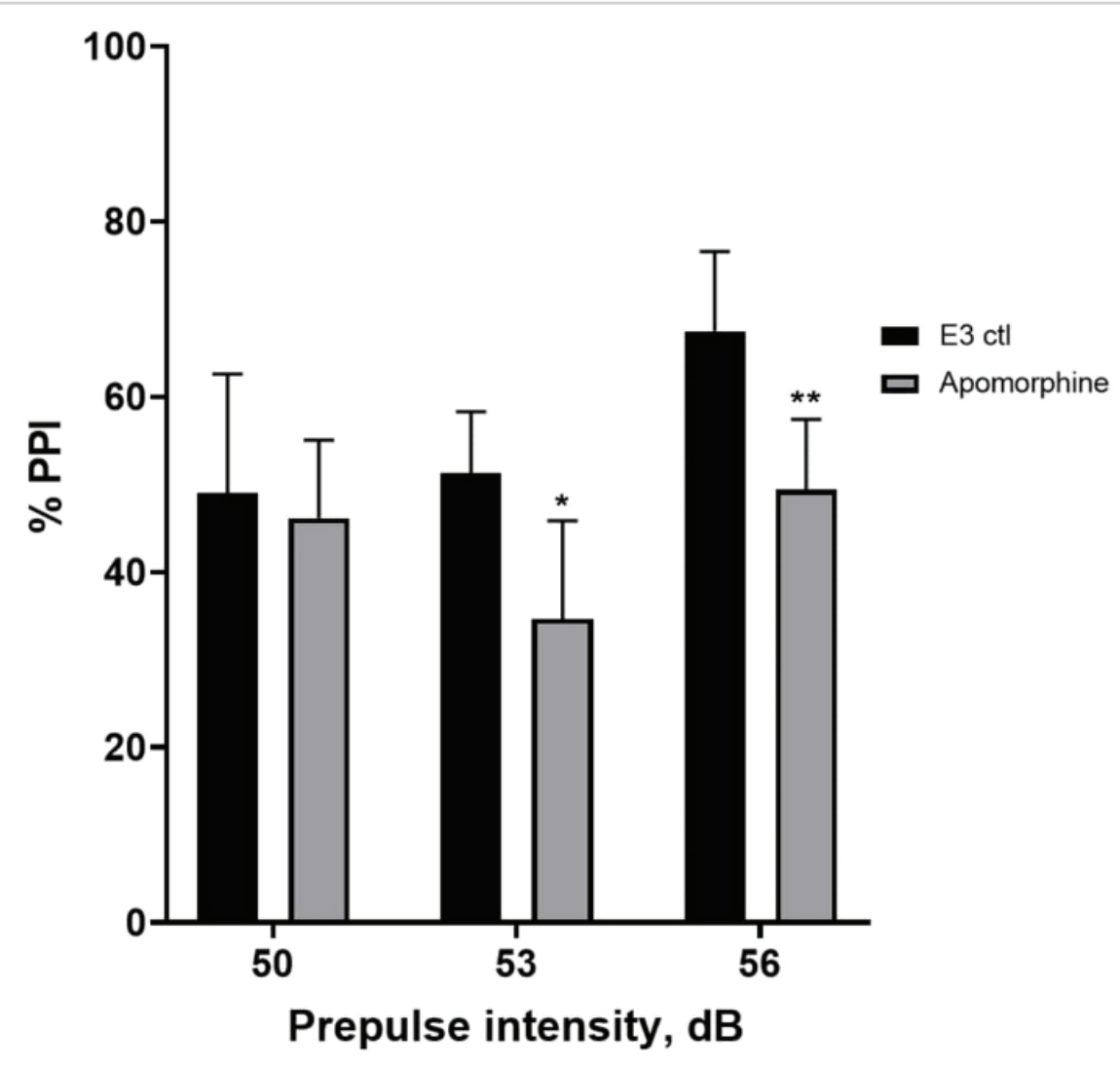

Figure 5: Apomorphine induced deficits in \%PPI. All data are presented as mean \pm S.D., $\mathrm{N}=4-5$ (16 larvae/group), statistically significant difference by Holm-Sidak's post-hoc test after two-way ANOVA. * $p=0.0126$, E3 ctl/apomorphine treated group at $53 \mathrm{~dB} ;{ }^{* *} \mathrm{p}=0.0044$, E3 ctl/apomorphine treated group at $56 \mathrm{~dB}$. Please click here to view a larger version of this figure. 


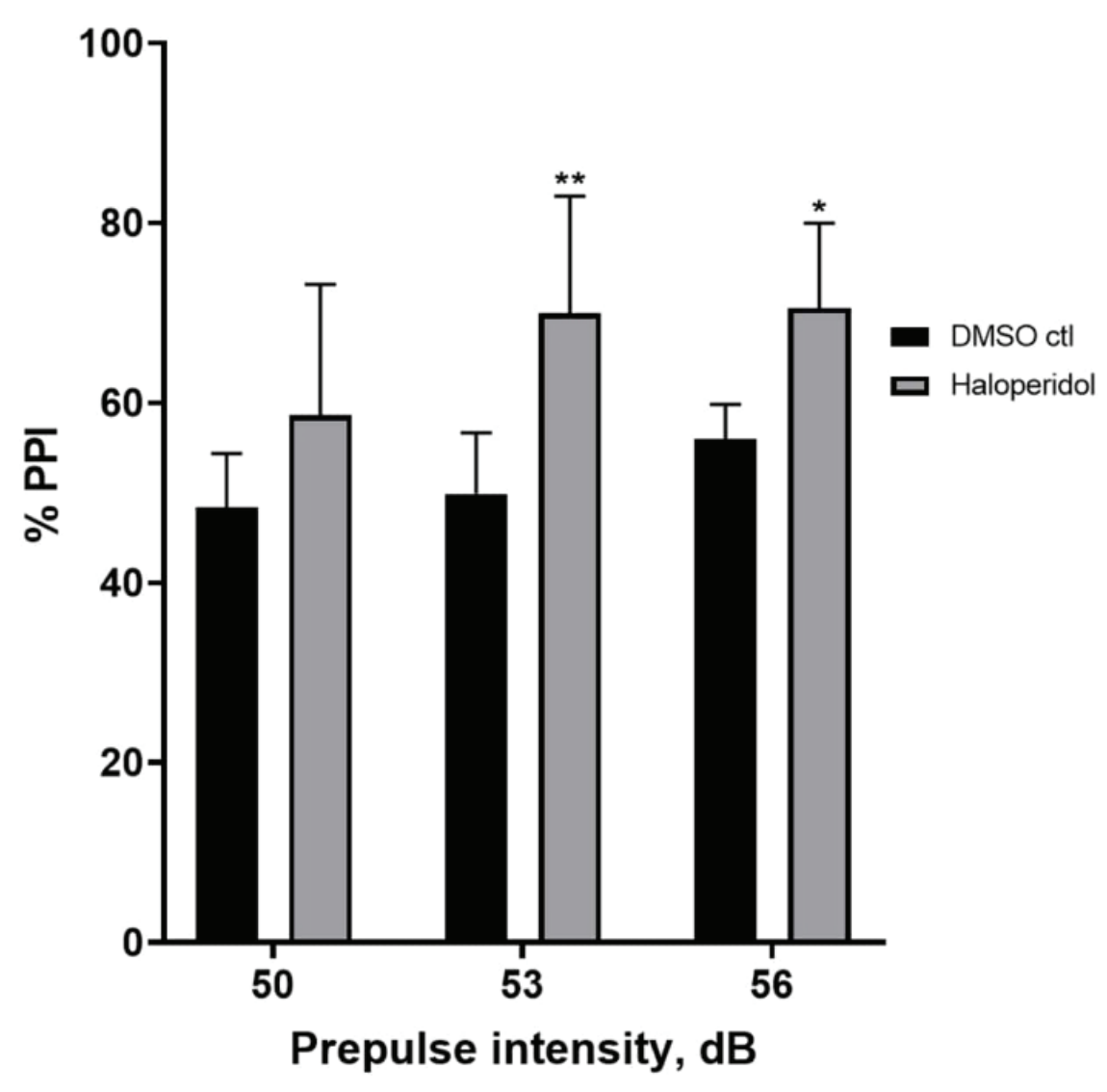

Figure 6: Haloperidol induced enhancement in \%PPI. All data are presented as mean \pm S.D., $N=4-5$ (16 larvae/group), statistically significant difference by Holm-Sidak's post-hoc test after two-way Anova. ${ }^{* *} \mathrm{p}=0.0048$, DMSO ct//apomorphine treated group at $53 \mathrm{~dB} ;{ }^{*} p=0.0348$, DMSO ct//apomorphine treated group at $56 \mathrm{~dB}$. Please click here to view a larger version of this figure. 


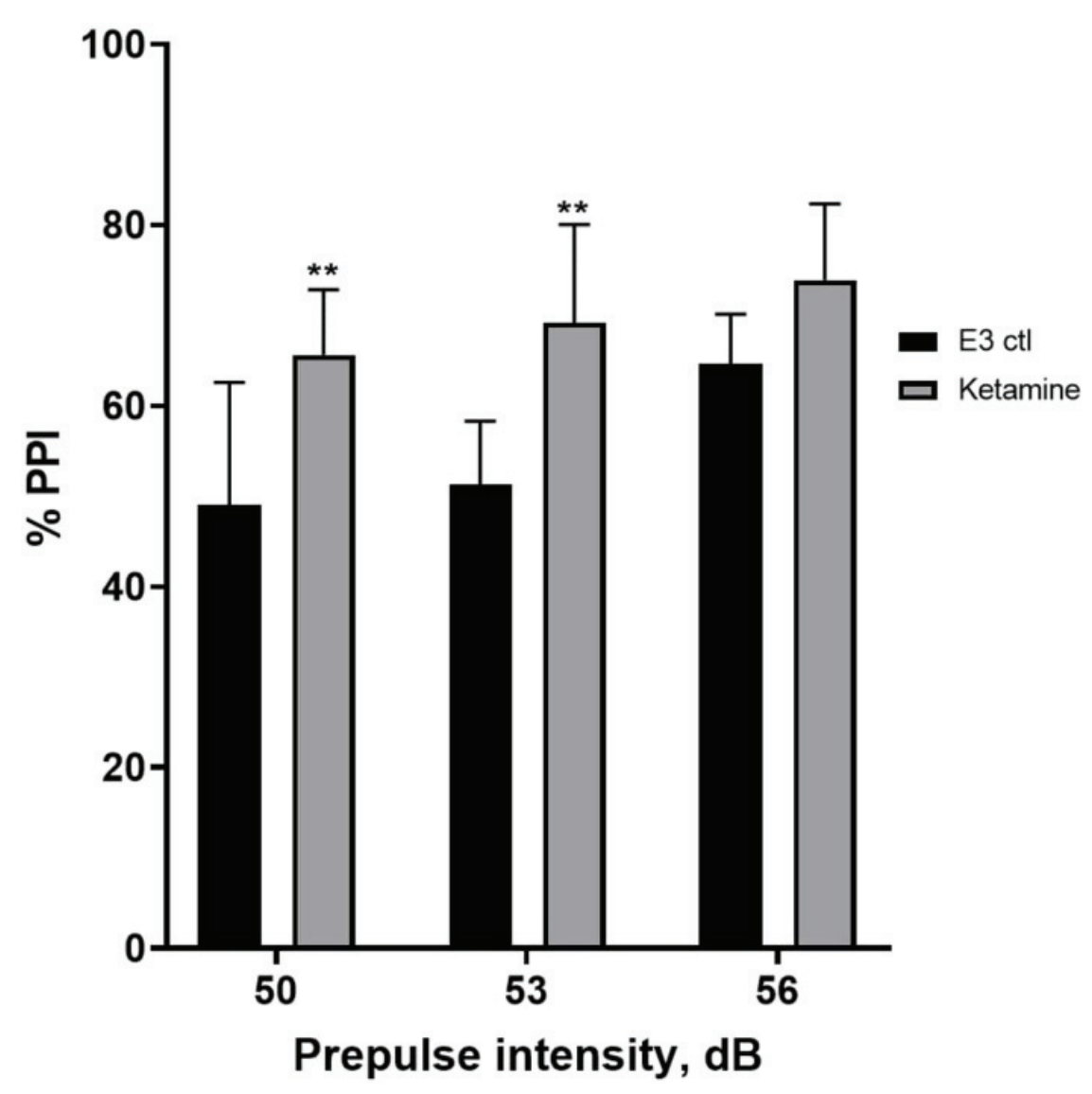

Figure 7: Ketamine induced enhancement in \%PPI. All data represented as mean \pm S.D, N = 4-5 (16 larvae/group), statistically significant difference by Holm-Sidak's post-hoc test after two-way Anova ** $p=0.0039$, E3 ctl/apomorphine treated group at $50 \mathrm{~dB},{ }^{* *} \mathrm{p}=0.0027$, E3 ctl/apomorphine treated group at $53 \mathrm{~dB}$. Please click here to view a larger version of this figure.

Supplementary Video 1: Representative video of larvae displaying a $\mathrm{C}$-start in response to $70 \mathrm{~dB}$ acoustic startle stimulus. Please click here to download this video.

\section{Supplementary Figure 1: Representative examples of} generated stimulus conditions using the PPI generator. (A) Stimulus alone trial, (B) pre-pulse inhibition trial (prepulse + pulse), (C) no stimulus trial to measure threshold baseline bend angle of unstimulated larvae. Please click here to download this figure.

\section{Discussion}

It is essential to validate any new behavioral assay system with the aim of improving and refining protocols for neurobehavioral research. In the current investigation, the ability of two commercially available systems and software to induce an acoustic startle response in zebrafish larvae and to detect and quantify previously described pharmacological modulation of such behaviors were assessed. 
A number of modifications and troubleshooting were performed to optimize the set-up. The default software for analysis of C-start responses was such that analysis automatically proceeded after the data for every experiment was acquired (22 trials/plate constituted an experiment). This reduced the number of plates that could be run per day, thus reducing the throughput (5 plates per day). To avoid this limitation, there was a need to de-couple the analysis software from the data collection process, which increased the throughput to an average of 10 plates per day. Thus, the decision to turn to an independent analysis software for nonlive analysis proved successful and more efficient. To avoid interference from shadows or other debris which introduces noise to the data, it is recommended to fill wells completely with medium, remove all bubbles and avoid food particles or similar which can be mistaken for larvae, thereby generating noise in the data. After calibration of the sound stimuli, the maximum intensity reachable by the amplifier system as captured by the $\mathrm{dB}$ meter was $85 \mathrm{~dB}$ re, while the initial background noise in the testing chamber was $60 \mathrm{~dB}$ re. This resulted in a narrow $\mathrm{dB}$ window in which to operate. Hence, it was critical to keep background noise as minimal as possible. To achieve this, parafon acoustics material (see Table of Materials) was used to build an additional layer of insulation around the test-chamber and an extra layer of insulation using a vocal booth bundle (see Table of Materials). With these layers of insulation, the background noise inside the testing chamber was successfully reduced from the initial $60 \mathrm{~dB}$ to $45 \mathrm{~dB}$ re.

Currently, one advantage of this set-up is that all the components are commercially available and as such, not limited to only a few labs. Individuals with limited knowledge in coding language can use it, as the protocol is rather easy to understand and follow. For example, by using the
PPI system, it was possible to deliver pulses and prepulses at varying inter-stimulus and inter-trial intervals, as well as capture larval responses to such stimuli. Once these behaviors were captured, they could be classified using the analysis software into responders and non-responders. The responder group was categorized as larvae that displayed a C-start of $30^{\circ}$ or more at a latency of $<50 \mathrm{~ms}$. In addition, the PPI response is modulated by drugs that target dopaminergic and glutamatergic signaling (reviewed by Geyer and colleagues ${ }^{27}$ ). Consistent with previous studies, apomorphine, a non-selective dopamine receptor agonist, reduced the pre-pulse inhibition of startle response in larval zebrafish, while haloperidol a dopamine antagonist enhanced the response. In larval zebrafish, ketamine has been shown to modulate PPI differentially based on the duration of the $|S|^{16}$. In the aforementioned study, larval PPI was enhanced at $30 \mathrm{~ms}$ but suppressed at $500 \mathrm{~ms}$ ISI when pre-treated with ketamine. Although this study did not use variable ISI, the observation that ketamine enhanced PPI at an ISI of $100 \mathrm{~ms}$, makes it comparable with the previous study's data when an ISI of $30 \mathrm{~ms}$ was used. The study demonstrated that by combining these commercially available systems, it is possible to perform the PPI assay and to reliably detect pharmacologically induced changes in the zebrafish larval PPI response. A limitation of the system is that the nosepoint feature tracked by the analysis software always falls on one of the eyes of the larvae, thereby creating a baseline angle. To overcome this, it is necessary to always determine the baseline bend angle of unstimulated larvae, which was found to be $\sim 30^{\circ}$ for larvae used in this study. Thus, forming the basis for the choice of $30^{\circ}$ as the threshold of what was considered a positive C-start response in startled larvae. If these points are taken into account, it should be possible to perform the PPI assay in any lab with access to the set-up equipment. This paper did not focus on categorizing 
the kinematics of startle response into short latency and long latency as reported earlier ${ }^{16}$, due to the scope of the variability of latency. Hence, only C-start responses $<50 \mathrm{~ms}$ after stimulus onset were used ${ }^{15}$.

Strain differences have been reported to influence zebrafish behavior in several assays $28,29,30,31$ as well as influence hearing sensitivity ${ }^{32}$. Hence, it is essential to determine the baseline bend angle of each strain tested. Since hearing sensitivities may also be different, it is crucial to determine baseline startle responses, the sound intensity most suited as either prepulse or startle stimulus for each strain and at what duration the stimulus is presented. The ISI is another parameter that should be carefully considered because some drugs can either enhance or reduce PPI based on the interval between the prepulse and startle stimulus onset ${ }^{16}$. The expectation is that, laboratories interested in studying cognitive function, neuropsychiatric disorders and hearing (auditory function) will find this PPI set-up and protocol useful in screening their pharmacological and/or genetic models. This protocol also provides a basis for high-throughput screening of compound libraries.

\section{Disclosures}

The authors declare no competing financial interests.

\section{Acknowledgments}

We thank Ana Tavara and João Paulo R. P. Santana for excellent fish care and invaluable help with testing and setting up of the soundproof booths, and Dr. Wietske van der Ent for initial support with setting up the EthoVision software. This study was funded by the Research Council of Norway (ISP, BIOTEK2021/ DigiBrain).

\section{References}

1. Gawel, K., Banono, N.S., Michalak, A., Esguerra, C.V. A critical review of zebrafish schizophrenia models: Time for validation? Neuroscience \& Biobehavioral Reviews. 107, 6-22 (2019).

2. Howe, K. et al. The zebrafish reference genome sequence and its relationship to the human genome. Nature. 496 (7446), 498-503 (2013).

3. Kalueff, A.V., Stewart, A.M., Gerlai, R. Zebrafish as an emerging model for studying complex brain disorders. Trends in Pharmacological Sciences. 35 (2), 63-75 (2014).

4. Panula, P. et al. The comparative neuroanatomy and neurochemistry of zebrafish CNS systems of relevance to human neuropsychiatric diseases. Neurobiology of Disease. 40 (1), 46-57 (2010).

5. Kokel, D., Peterson, R.T. Chemobehavioural phenomics and behaviour-based psychiatric drug discovery in the zebrafish. Briefings in Functional Genomics and Proteomics. 7 (6), 483-490 (2008).

6. Basnet, R.M., Zizioli, D., Taweedet, S., Finazzi, D., Memo, M. Zebrafish Larvae as a Behavioral Model in Neuropharmacology. Biomedicines. 7 (1), 23 (2019).

7. Henry, J., Wlodkowic, D. Towards High-Throughput Chemobehavioural Phenomics in Neuropsychiatric Drug Discovery. Marine Drugs. 17 (6) (2019).

8. Behavioural measurement system | Laval zebrafish | Drosophila | Daphnia | insects | Products | Zantiks. at <https://zantiks.com/products/zantiks-mwp>. (2020).

9. Product List - ViewPoint. at <http://www.viewpoint.fr/en/ products>. (2020).

10. Observation chamber for zebrafish research DanioVision. Observation chamber for zebrafish research 
- DanioVision. at <https://www.noldus.com/daniovision/ observation-chamber>. (2020).

11. EthoVision XT - Video tracking software | Noldus. EthoVision XT - Video tracking software | Noldus. at <https://www.noldus.com/ethovision-xt>. (2020).

12. Gould, T.D., Gottesman, I.I. Psychiatric endophenotypes and the development of valid animal models. Genes, Brain and Behavior. 5 (2), 113-119 (2006).

13. van den Buuse, M. Modeling the Positive Symptoms of Schizophrenia in Genetically Modified Mice: Pharmacology and Methodology Aspects. Schizophrenia Bulletin. 36 (2), 246-270 (2010).

14. Acoustic startle modification as a tool for evaluating auditory function of the mouse: Progress, pitfalls, and potential. - Abstract - Europe PMC. at <https:// europepmc.org/article/PMC/5446932>. (2020).

15. Bhandiwad, A.A., Zeddies, D.G., Raible, D.W., Rubel, E.W., Sisneros, J.A. Auditory sensitivity of larval zebrafish (Danio rerio) measured using a behavioral prepulse inhibition assay. Journal of Experimental Biology. 216 (18), 3504-3513 (2013).

16. Burgess, H.A., Granato, M. Sensorimotor Gating in Larval Zebrafish. Journal of Neuroscience. 27 (18), 4984-4994 (2007).

17. Eaton, R.C., Farley, R.D., Kimmel, C.B., Schabtach, E. Functional development in the mauthner cell system of embryos and larvae of the zebra fish. Journal of Neurobiology. 8 (2), 151-172 (1977).

18. SR-LAB - San Diego Instruments Startle Response. San Diego Instruments. at <https://sandiegoinstruments.com/ product/sr-lab-startle-response/>. (2020).
19. Startle packages - Med Associates Inc. Med Associates Inc. at <https://www.med-associates.com/productcategory/acoustic-startle-reflex-packages/>. (2020).

20. Startle response \& Pre-pulse inhibition test| O'HARA \& CO.,LTD. at <https://ohara-time.co.jp/products/startleresponse-pre-pulse-inhibition-test/>. (2020).

21. Thyme, S.B. et al. Phenotypic Landscape of Schizophrenia-Associated Genes Defines Candidates and Their Shared Functions. Cell. 177 (2), 478-491.e20 (2019).

22. Privat, M. et al. Sensorimotor Transformations in the Zebrafish Auditory System. Current Biology. 29 (23), 4010-4023.e4 (2019).

23. Gerlai, R. Reproducibility and replicability in zebrafish behavioral neuroscience research. Pharmacology Biochemistry and Behavior. 178, 30-38 (2019).

24. Add-on - Fast camera 1000 FPS - ViewPoint. at <http://www.viewpoint.fr/app.php/en/p/equipment/addon-fast-camera>. (2020).

25. Aleström, P. et al. Zebrafish: Housing and husbandry recommendations: Laboratory Animals. (2019).

26. Bhandiwad, A.A., Sisneros, J.A. Revisiting Psychoacoustic Methods for the Assessment of Fish Hearing. Fish Hearing and Bioacoustics: An Anthology in Honor of Arthur N. Popper and Richard R. Fay. 157-184 (2016).

27. Geyer, M.A., Krebs-Thomson, K., Braff, D.L., Swerdlow, N.R. Pharmacological studies of prepulse inhibition models of sensorimotor gating deficits in schizophrenia: a decade in review. Psychopharmacology. 156 (2-3), 117-154 (2001). 
28. Lange, M. et al. Inter-Individual and Inter-Strain Variations in Zebrafish Locomotor Ontogeny. PLOS ONE. 8 (8), e70172 (2013).

29. Liu, X. et al. Strain-dependent differential behavioral responses of zebrafish larvae to acute MK-801 treatment. Pharmacology Biochemistry and Behavior. 127, 82-89 (2014).

30. Loucks, E., Carvan, M.J. Strain-dependent effects of developmental ethanol exposure in zebrafish. Neurotoxicology and Teratology. 26 (6), 745-755 (2004).

31. Bos, R. van den et al. Further characterisation of differences between $T L$ and $A B$ zebrafish (Danio rerio): Gene expression, physiology and behaviour at day 5 of the larval stage. PLOS ONE. 12 (4), e0175420 (2017).

32. Monroe, J.D. et al. Hearing sensitivity differs between zebrafish lines used in auditory research. Hearing research. 341, 220-231 (2016). 
Materials List for

\section{Pharmacological Validation of the Prepulse Inhibition of Startle Response in Larval Zebrafish using a Commercial Automated System and Software}

Nancy Saana Banono ${ }^{1,2}$, Camila V. Esguerra ${ }^{1,2}$

${ }^{1}$ Chemical Neuroscience Group, Centre for Molecular Medicine Norway (NCMM), Faculty of Medicine, University of Oslo ${ }^{2}$ Section for Pharmacology and Pharmaceutical Biosciences, Department of Pharmacy, Faculty of Mathematics and Natural Sciences, University of Oslo

\section{Corresponding Author}

Camila V. Esguerra

c.v.esguerra@ncmm.uio.no

Date Published

July 2, 2020

\section{Citation}

Banono, N.S., Esguerra, C.V. Pharmacological Validation of the Prepulse Inhibition of Startle Response in Larval Zebrafish using a Commercial Automated System and Software. J. Vis. Exp. (), e61423, doi:10.3791/61423 (2020).

DOI

URL

$10.3791 / 61423$

\section{Materials}

\begin{tabular}{|l|l|l|l|}
\hline Name & Company & Catalog Number & Comments \\
\hline Apomorphine & Sigma Aldrich & A4393 & Dopamine agonist \\
\hline dB meter & PCE instruments & PCE-MSM 4 & For measuring stimulus intensity \\
\hline DMSO & Sigma Aldrich & D8418 & For dissolving organic solutes \\
\hline Dynavox Amplifier & Dynavox & CS-PA1 MK & For delivering acoustic stimuli \\
\hline EthoVision XT & Noldus, Netherlands & EthoVision XT, version 14 & Automated tracking software \\
\hline GraphPad Prism & GraphPad Software & Version 8 & Statistical analysis software \\
\hline Haloperidol & Sigma Aldrich & H1512 & Dopamine antagonist \\
\hline Ketamine & Sigma Aldrich & Y0000450 & NMDA receptor antagonist \\
\hline parofon acoustics materials & Paroc & 8528308 & $\begin{array}{l}\text { Helps reduce background noise in } \\
\text { the test cabinet }\end{array}$ \\
\hline t.akustik Vocal Booth Bundle & Thormann, Germany & 458543 & $\begin{array}{l}\text { Helps reduce background noise in } \\
\text { the test cabinet }\end{array}$ \\
\hline ZebraBox Revo with PPI add-ons & ViewPoint, France & ZebraBox Revo with PPI add-ons & Includes hardware and software \\
\hline
\end{tabular}


Supplementary Figure 1: Representative examples of generated stimulus conditions using the PPI generator. (A) Stimulus alone trial, (B) pre-pulse inhibition trial (pre-pulse + pulse), (C) no stimulus trial to measure threshold baseline bend angle of unstimulated larvae.
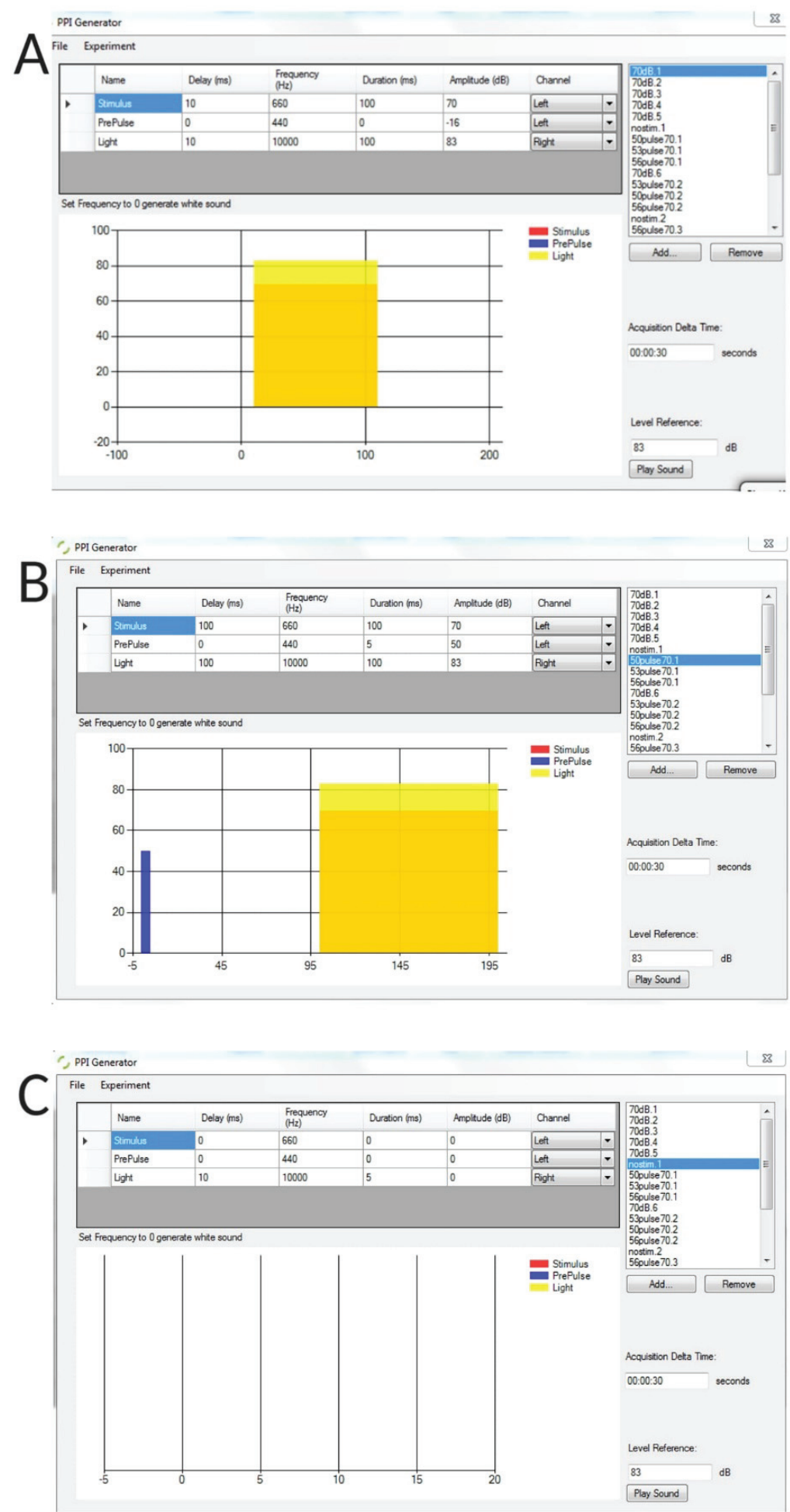


\section{Paper III}

\section{Zebrafish larvae carrying a splice variant mutation in cacna1d: a new model for schizophrenia-like behaviours?}

Banono, Nancy Saana; Gawel, Kinga Aurelia; De Witte, Linus \& Esguerra, Camila V.

Published in Molecular Neurobiology, September 2020, Volume 58, pp. 877-894, DOI: 10.1007/s12035-020-02160-5 



\title{
Zebrafish Larvae Carrying a Splice Variant Mutation in cacna1d: A New Model for Schizophrenia-Like Behaviours?
}

\author{
Nancy Saana Banono ${ }^{1}$ (D) $\cdot$ Kinga Gawel $^{1,2}$ (D) $\cdot$ Linus De Witte $^{3} \cdot$ Camila V. Esguerra $^{1,4}$ (D)
}

Received: 6 July 2020 / Accepted: 2 October 2020

(C) The Author(s) 2020

\begin{abstract}
Persons with certain single nucleotide polymorphisms (SNPs) in the CACNA1D gene (encoding voltage-gated calcium channel subunit alpha 1-D) have increased risk of developing neuropsychiatric disorders such as bipolar, schizophrenia and autism. The molecular consequences of SNPs on gene expression and protein function are not well understood. Thus, the use of animal models to determine genotype-phenotype correlations is critical to understanding disease pathogenesis. Here, we describe the behavioural changes in larval zebrafish carrying an essential splice site mutation (sa17298) in cacnalda. Heterozygous mutation resulted in 50\% reduction of splice variants 201 and 202 (haploinsufficiency), while homozygosity increased transcript levels of variant 201 above wild type (WT; gain-of-function, GOF). Due to low homozygote viability, we focused primarily on performing the phenotypic analysis on heterozygotes. Indeed, cacnalda ${ }^{\text {sal7298/WT }}$ larvae displayed hyperlocomotion-a behaviour characterised in zebrafish as a surrogate phenotype for epilepsy, anxiety or psychosis-like behaviour. Follow-up tests ruled out anxiety or seizures, however, as neither thigmotaxis defects nor epileptiform-like discharges in larval brains were observed. We therefore focused on testing for potential "psychosis-like" behaviour by assaying cacnalda ${ }^{\text {sa17298/WT }}$ larval locomotor activity under constant light, during light-dark transition and in startle response to dark flashes. Furthermore, exposure of larvae to the antipsychotics, risperidone and haloperidol reversed cacnalda-induced hyperactivity to WT levels while valproate decreased but did not reverse hyperactivity. Together, these findings demonstrate that cacnalda haploinsufficiency induces behaviours in larval zebrafish analogous to those observed in rodent models of psychosis. Future studies on homozygous mutants will determine how cacnald GOF alters behaviour in this context.
\end{abstract}

Keywords Zebrafish $\cdot C A C N A 1 D \cdot$ Neurobehaviour $\cdot$ Neuropsychiatric disorders $\cdot$ Schizophrenia $\cdot$ Psychosis

Electronic supplementary material The online version of this article (https://doi.org/10.1007/s12035-020-02160-5) contains supplementary material, which is available to authorized users.

Camila V. Esguerra

c.v.esguerra@ncmm.uio.no

Linus De Witte

https://orcid.org/0000-0002-2374-637X

1 Chemical Neuroscience Group, Centre for Molecular Medicine Norway (NCMM), Faculty of Medicine, University of Oslo, Gaustadalléen 21, Forskningsparken, 0349 Oslo, Norway

2 Department of Experimental and Clinical Pharmacology, Medical University of Lublin, Jaczewskiego Str. 8b, 20-090 Lublin, Poland

3 Pharmaceutical and Biological Sciences, AP Hogeschool Antwerpen, Antwerp, Belgium

4 School of Pharmacy, Faculty of Mathematics and Natural Sciences, University of Oslo, Sem Sælandsvei 24, 0371 Oslo, Norway

\section{Introduction}

Schizophrenia (SCZ) is a highly heritable and polygenic neuropsychiatric disorder. The high prevalence of SCZ worldwide has made it a burgeoning public health concern. Genome-wide association studies (GWAS) have led to the initial identification of small nucleotide polymorphisms (SNPs) in about 108 risk loci linked to SCZ [1]. The majority of SNPs implicated in SCZ and other psychiatric disorders are located in noncoding (intergenic and intronic) regions of the genome [2]. To date, the functional significance of these noncoding variants remains unclear, thus obviating the need for establishment and validation of genetic animal models to elucidate underlying disease mechanisms associated with SCZrisk genes. Nevertheless, SNPs in the intronic regions of genes are thought to result in changes in gene expression levels by altering splicing (via splice donor or acceptor sites) and transcription (via disruption of gene regulatory elements such as 
transcription factors, promoters, enhancers and/or suppressors) [3, 4]. For example, there is evidence that rs100637 (a $\mathrm{SCZ}$-associated SNP, located in intron 3 of the CACNA1C gene) is associated with major depressive disorder (MDD), SCZ and attention deficit hyperactivity disorder, leads to changes in CACNA1C expression in both human carriers [5, 6] and induced human neurons [7]. In humans, broader phenotypes are present such as deficits in prepulse inhibition (PPI), latent inhibition and sleep disturbance in some SNP carriers, mimicking a broad spectrum of neuropsychiatric disorders $[2,8]$.

CACNA1D encodes the $\alpha 1$ subunit of the $\mathrm{CaV}_{1.3}$ voltagegated L-type isoform. In the central nervous system (CNS) of humans, the $\mathrm{CaV}_{1.3}$ isoform is expressed in neuroendocrine cells, cerebral cortex, habenula, hippocampus, thalamus and basal ganglia, where it plays essential roles in consolidation of fear memory, drug-seeking behaviours and fine tuning of various elements of neuronal plasticity [9-12]. Mutations in CACNA1D have been implicated in neurodevelopmental and neuropsychiatric diseases [13-15]. For instance, persons with SNPs in CACNA1D have increased risk of developing bipolar disorder (BP), attention deficit hyperactivity disorder, SCZ, autism spectrum disorder (ASD) or MDD [1, 13, 16-21]. In one study, two single nucleotide variants in $C A C N A 1 D$ were found in separate cohorts of bipolar patients [22]. Although a study in the Han Chinese population did not find an association of CACNAID with SCZ [23], other subsequent studies did identify such an association $[8,21]$. Furthermore, wholeexome sequencing revealed variants in the CACNA1D coding region to be linked to $\mathrm{ASD}$, depression, anxiety, fear and seizures $[17,18]$.

Homozygous $\mathrm{CaV}_{1.3}$ knockout mice are deaf and have impaired cardiac function [24], display antidepressant-like phenotypes [25] and have impaired consolidation of fear in the Pavlovian test [26]. In zebrafish (Danio rerio), cacnald is duplicated into cacnalda and cacnaldb, which encode $\mathrm{CaV}_{1.3 \mathrm{a}}$ and $\mathrm{CaV}_{1.3 \mathrm{~b}}$ respectively [27]. The amino acid sequence of zebrafish Cacnalda and Cacnaldb is $77 \%$ and $33 \%$ homologous to human CACNA1D respectively [28, 29]. Previous zebrafish studies have focused primarily on characterising the role of cacnald in auditory and vestibular function [27, 30-32]. Homozygous cacnalda nonsense mutants display a classical auditory-vestibular phenotype reminiscent of "circler mutants" (i.e. circular swimming due to impaired balance) $[27,33,34]$.

Interestingly, the essential splice site mutation described in this study was found to mimic both gene haploinsufficiency in the heterozygous state and gain-of-function (GOF) in the homozygous state. As homozygous mutant survival was low (i.e. very small sample size), we focused the majority of our analysis on heterozygotes, thus investigating the effects of cacnalda haploinsufficiency on larval zebrafish behaviour and electroencephalography (EEG) patterns. We profiled for anxiety-, seizure- or psychosis-like behaviours, by performing the following assays: PPI response, locomotor activity (constant light), light-dark transition test, thigmotaxis and startle response to dark flashes. We also tested the neuromodulatory activity of risperidone (RISP), an atypical antipsychotic drug [35]; haloperidol (HALO), a dopamine antagonist used as a typical antipsychotic drug [36]; and valproic acid (VPA), an anti-seizure drug and mood stabiliser [37, 38], to determine whether cacnald mutant larvae would respond in a similar manner as SCZ, BP and/or epilepsy patients/animal models. Finally, EEG recordings were performed to establish whether the hyperlocomotion observed at earlier developmental time points resulted from epileptiform-like brain activity.

\section{Materials and Methods}

\section{Ethical Considerations}

Approval by the Norwegian Food Safety Authority via its experimental animal administration's supervisory and application system (FOTS-ID 15469 and 23935) was obtained prior to animal experimentation. Also, compliance with the National Institute of Health Guidelines for the Care and Use of Laboratory Animals and the European Community Council Directive of November 2010 for Care and Use of Laboratory Animals (Directive 2010/63/EU), as well as the ARRIVE guidelines, were adhered to during all experiments.

\section{Zebrafish Strains and Husbandry}

The sa17298 mutant line was generated by N-ethyl-Nnitrosourea (ENU) mutagenesis within the Zebrafish Mutation Project (Sanger, UK). The line carries a point mutation in cacnalda, which spans an essential splice site [for details see: [39]]. Fertilised sa17298 embryos were obtained from the Zebrafish International Resource Center (Eugene, Oregon, USA) and raised to adulthood, genotyped and the heterozygous animals outcrossed to $\mathrm{AB}$ wild-type (WT) zebrafish for three generations. Animals were raised under controlled conditions described by [40], in a 14-/10-h light/dark cycle at $28.5^{\circ} \mathrm{C}$.

Fertilised eggs from natural spawning of adult fish lines were collected, transferred to petri dishes (density $N=50-70)$, filled with embryo medium (17 mM NaCl, $2 \mathrm{mM} \mathrm{KCl}, 1.8 \mathrm{mM} \mathrm{Ca}(\mathrm{NO} 3), 2,0.12 \mathrm{mM} \mathrm{MgSO} 4$, $1.5 \mathrm{mM}$ HEPES buffer $\mathrm{pH} 7.1-7.3$ and $0.6 \mu \mathrm{M}$ methylene blue). These petri dishes were stored in an incubator with a 14/-10-h light/dark cycle at $28.5{ }^{\circ} \mathrm{C}$. The medium was refreshed daily until larvae reached 7-day post-fertilisation (dpf). 


\section{Genotyping}

Adult fin clip tissue or whole larvae were snap frozen in liquid nitrogen. DNA from fin clip tissue or larval sample was extracted using PCR extraction buffer (10 mM Tris ( $\mathrm{pH} 8.0)$, $2 \mathrm{mM}$ EDTA, $0.2 \%$ Triton $\mathrm{X}-100$ ) and proteinase $\mathrm{K}$ $(200 \mu \mathrm{g} / \mathrm{ml})$. DNA was amplified using DreamTaq DNA polymerase (EP0702, Thermofischer) according to the manufacturer's instructions in a $20 \mu \mathrm{L}$ final volume. The forward primer: 5' TGTGCTGGTGTTGTGTGTG 3' and reverse primer: 5' TCAAGCCAGGAAGTACTGAAG 3' were used with the following cycling conditions: Step 1: Initial denaturation $95{ }^{\circ} \mathrm{C}, 1 \mathrm{~min}$; Step 2: Denaturation $95^{\circ} \mathrm{C}, 30 \mathrm{~s}$; Step 3: Annealing temperature $59^{\circ} \mathrm{C}, 30 \mathrm{~s}$; Step 4: Extension $72^{\circ} \mathrm{C}$, $1 \mathrm{~min}$; Step 5: Repeat steps 2-4, $34 \times$; Step 6: Final extension $72{ }^{\circ} \mathrm{C}, 1 \mathrm{~min}$. This resulted in a 196 base pair (bp) amplicon. The PCR product was digested using the restriction enzyme, BstEII (R0162M, New England BioLabs). The bands were visualised using $2 \%$ agarose gel electrophoresis and Sybr Safe (S33102, Thermofischer) as the DNA intercalating agent. Observers conducting behavioural tests were blind to the genotype of the larvae. Hence, larvae were genotyped after each experiment.

\section{Morphological Assessment}

Larvae were photographed using a Leica M205 FA stereomicroscope and assembled using Adobe Photoshop 2020. All pictures were taken at $5 \mathrm{dpf}$ at the same resolution for comparison.

\section{Drugs}

The following drugs were used: $5 \mu \mathrm{M}$ RISP, $50 \mu \mathrm{M}$ HALO and $100 \mu \mathrm{M}$ VPA. All drugs were dissolved in DMSO at a final concentration of $0.5 \% \mathrm{v} / \mathrm{v}$ DMSO for the HALO and VPA groups but $0.1 \% \mathrm{v} / \mathrm{v}$ DMSO for the RISP-treated group. The appropriate vehicle controls were prepared by dissolving DMSO in zebrafish E3 medium. DMSO and HALO were purchased from Sigma-Aldrich, RISP from TOCRIS and VPA from Sanofi Aventis. The drug concentrations used were selected based on previously published studies [35, 36, 41].

\section{Behavioural Tests and Material}

Larvae were transferred to the behavioural analysis room at least $1 \mathrm{~h}$ prior to experimentation to allow animals to acclimate. Single larvae were gently transferred from the petri dishes to individual wells of a 24-well plate (diameter $16.2 \mathrm{~mm}$ ) except when stated otherwise. Automated videotracking of larval behaviour was carried out using the ZebraBox hardware and ZebraLab software (Viewpoint, Lyon, France). Light-dark test and thigmotaxis measurement were carried out using the same larvae by simply defining the inner and outer regions of each well of the tracking plate (the inner zone was of diameter $8 \mathrm{~mm}$ while the distance from the inner zone relative to the outer zone was $4 \mathrm{~mm}$ ). A different set of larvae were used for the startle response test. Except for the startle response to dark flashes test, all other experiments were replicated two or three times and the results pooled together.

\section{Locomotor Activity and Thigmotaxis}

Larvae were allowed to acclimate to the test chamber (ZebraBox) for $15 \mathrm{~min}$ in the dark, followed by $10 \mathrm{~min}$ of tracking in either $100 \%$ light or dark ( $0 \%$ light). For the light-dark transition test, after the acclimation, 10 min each of tracking in the following conditions: (1) $100 \%$ light followed by (2) dark in succession. The locomotor activity of larvae was measured as the total distance travelled in millimetres (mm) over a 10 -min period.

For thigmotaxis, the following parameters were measured: (1) distance spent in inner zone, (2) distance spent in outer zone and (3) distance in entire arena. Thigmotaxis was calculated as percentages using the following formula [42]:

Thigmotaxis $(\%$ distance in outer zone $)=\frac{(\text { Distance outer zone })}{(\text { Distance outer }+ \text { inner zone })} \times 100$

\section{Startle Response to Dark Flashes}

To trigger a startle response in larvae, 150-ms-long dark flashes were used, and this was repeated severally to assess the ability of the larvae to habituate. The startle response test was made up of three steps: (1) 15-min acclimatisation period in $100 \%$ illuminated test chamber, (2) 10-min baseline locomotor tracking in the fully illuminated test chamber, (3) followed by the presentation of 30 dark flashes repeated every $3 \mathrm{~s}$ [43].

\section{Locomotor Activity of Drug-Treated Larvae}

Larvae were pre-exposed $30 \mathrm{~min}$ to RISP, HALO or VPA and their respective vehicle control groups, transferred to the test chamber and tracking started immediately for $4 \mathrm{~h}$ at a 30 -min integration period. Only tracks after $2 \mathrm{~h}$ were used for analysis. For the 24-h RISP-treated group, larvae were pre-exposed to treatment $22 \mathrm{~h}$ then transferred to the test chamber and tracked immediately for $4 \mathrm{~h}$ with the tracks after $2 \mathrm{~h}$ used for analysis. Larvae were tracked in 100\% light condition and the locomotor activity of larvae were measured as the total distance travelled in millimetres over a 30-min period. Vehicle control and drug-treated larvae were from the same clutch. 


\section{Acoustic Startle Response (ASR) and Prepulse Inhibition (PPI)}

Acoustic stimuli were delivered using the ZebraBox Revo and the behavioural response analysed with EthoVision software as previously described [44] using larvae aged 6 dpf. All experiments were performed by placing larvae individually in each well of a custom-made plexiglass plate of 33 wells in a 96 format. Acclimation was for 5 min prior to the onset of experiment in a 100-Lx illuminated chamber. The startle stimulus was $100 \mathrm{~ms}$ at $660 \mathrm{~Hz}$ while the prepulse stimulus was $5 \mathrm{~ms}$ at $440 \mathrm{~Hz}$. A 100-ms inter-stimulus interval (ISI) was used for PPI experiments.

\section{Zebrafish EEG Recordings}

The EEG recordings were performed as previously described by Afrikanova et al. [45]. Epileptiform-like discharges were detected by inserting a glass electrode filled with artificial cerebrospinal fluid (124 mM NaCl, $2 \mathrm{mM} \mathrm{KCl,} 2 \mathrm{mM}$ $\mathrm{MgSO}_{4}, 2 \mathrm{Mm} \mathrm{CaCl}_{2}, 1.25 \mathrm{mM} \mathrm{KH}_{2} \mathrm{PO}_{4}, 26 \mathrm{mM} \mathrm{NaHCO}_{3}$, $10 \mathrm{mM}$ glucose) into the optic tectum of individual 6-dpf zebrafish larvae for $20 \mathrm{~min}$ (MultiClamp 700B amplifier, Digidata 1550 digitiser, Axon instruments, USA). The larvae were restrained with the aid of a thin layer of $2 \%$ low melting point agarose. The Clampfit version 10.6.2 software (Molecular Devices Corporation, USA) was used for processing the EEG recordings. The data were analysed manually by a trained observer, blind to the genotype of the larvae.

\section{RNA Isolation and CDNA Synthesis}

Total RNA was isolated from a pool of 6-dpf larvae $(N=25$ larvae, 3 replicates/group) using the Invitrogen PureLink RNA Mini Kit (12183018A, Thermofischer). A Nanodrop 1000 spectrophotometer and agarose bleach gel described by [46] was used to assess the quality/integrity of the RNA. cDNA was synthesised from $1 \mu \mathrm{g}$ RNA with oligo dT primers using the Invitrogen SuperScript IV Reverse Transcriptase (18090050, Thermofischer) according to the manufacturer's instructions.

\section{Reverse Transcriptase and Quantitative Polymerase Chain Reaction}

rt-PCR was performed for 40 cycles using Taq-polymerase (EP0406, Thermofischer) in a $20 \mu \mathrm{l}$ final reaction using $1 \mu \mathrm{l}$ of cDNA template. PCR products were visualised on $2 \%$ agarose gels. The qPCR reactions were performed with Power SYBR Green Master Mix (4368702, Thermofischer) on the CFX384 Touch Real-Time PCR Detection System (1855485, Bio Rad). All primers were synthesised by Sigma with melting temperature $>60{ }^{\circ} \mathrm{C}$. Samples were run in triplicates in a
$20 \mu \mathrm{l}$ final volume containing $10 \mu \mathrm{l} 2 \times$ sybr green, $1 \mu \mathrm{l}$ $10 \mu \mathrm{M}$ forward + reverse primer and $9 \mu \mathrm{l}$ 1:180 cDNA dilution. The following cycling parameters were used: $95^{\circ} \mathrm{C}$ for $10 \mathrm{~min}$, and then 40 cycles of $95^{\circ} \mathrm{C}$ for $15 \mathrm{~s}, 60^{\circ} \mathrm{C}$ for $1 \mathrm{~min}$. To check for the presence of non-specific products and/or primer dimers, a dissociation step was performed at the end of each amplification phase from 65 to $95^{\circ} \mathrm{C}$, at $0.5^{\circ} \mathrm{C}$ increment for $5 \mathrm{~s}$. The comparative $\Delta \Delta \mathrm{CT}$ method was calculated using the Bio Rad CFX Manager 3.1. The endogenous genes glyceraldehyde-3-phosphate dehydrogenase (gapdh) and ribosoamal protein $S 18$ (rps18) were used for data normalisation. See Suppl. Table 1 for the list of rt-/q-PCR primer sequences.

\section{Statistical Analysis}

Statistical analysis and figures generation were performed with the aid of GraphPad Prism 8.4.1 (San Diego, CA, USA). For behavioural experiments, a two-way analysis of variance (ANOVA) (factors: phase/treatment and genotype), followed by Tukey's post hoc test, was used. For EEG experiment, Student's $t$ test was applied. Unpaired Student's $t$ test or its equivalent non-parametric test, Mann Whitney $U$, was performed where necessary. Statistical significance was established at $p<0.05$. In figures, dots represent individual measurements.

\section{Results}

\section{Genotyping and Molecular Consequences of the cacna1da Splice Site Mutation}

As mentioned earlier, the amino acid sequence of zebrafish Cacnalda and Cacnaldb are $77 \%$ and $33 \%$ homologous to human CACNA1D protein respectively. cacnalda has two transcript variants while cacnaldb has a single transcript $[28,29]$. The cacnalda transcript variant 202 spans 47 exons (Fig. 1a), whereas variant 201 spans 48 exons (not shown). The sa17298 allele contains a single point mutation $(\mathrm{G}>\mathrm{A})$ at the first residue of intron 20-21 of the cacnalda-202 transcript variant (Fig. 1b). Importantly, it does not alter the sequence of variant 201. To identify mutants, PCR of larval and/or adult fin clip tissue was digested using BstEII restriction enzyme. The BstEII restriction site is absent in the mutant allele. Hence, $2 \%$ agarose gel electrophoresis of the digested product resulted in one band for homozygous sa17298 ( 196 bp), two bands for WT $s a 17298(\sim 70$ and $129 \mathrm{bp})$ and three bands for heterozygous sal 7298 ( 70,129 and 196 bp) (Fig. 1c).

To assess whether the splice site mutation resulted in exon skipping, rt-PCR, followed by agarose gel electrophoresis, was performed using primers spanning exons 20-22 of cacnalda transcript variant 202. No difference in product size 
was observed between WT and homozygous mutants, thus suggesting that the mutation did not result in exon skipping. Notably, however, a difference in signal intensity of the rtPCR amplicons was observed (Fig. 1d). Given the difference in signal intensity obtained from WT and homozygotes, we next tested whether the mutation altered mRNA levels. We performed qPCR analysis using three cacnalda primer sets. The first primer set [cacnalda_qPCR] amplified a region with high complementarity between the 201 and 202 transcript variants, the second primer set [E20-22_v202] targeted only the 202 transcript while the third primer set [E22-24_v201] targeted only the 201 transcript (Suppl. Fig. 1). qPCR analysis revealed an overall reduction of cacnalda mRNA levels (ca. $50 \%$ and $40 \%$ ) in heterozygous and homozygous mutants respectively. Primer-specific amplification of variant 202 showed a 50\% and $90 \%$ decrease in transcript 202 levels in heterozygous and homozygous mutants respectively. Interestingly, when primers specifically targeting transcript
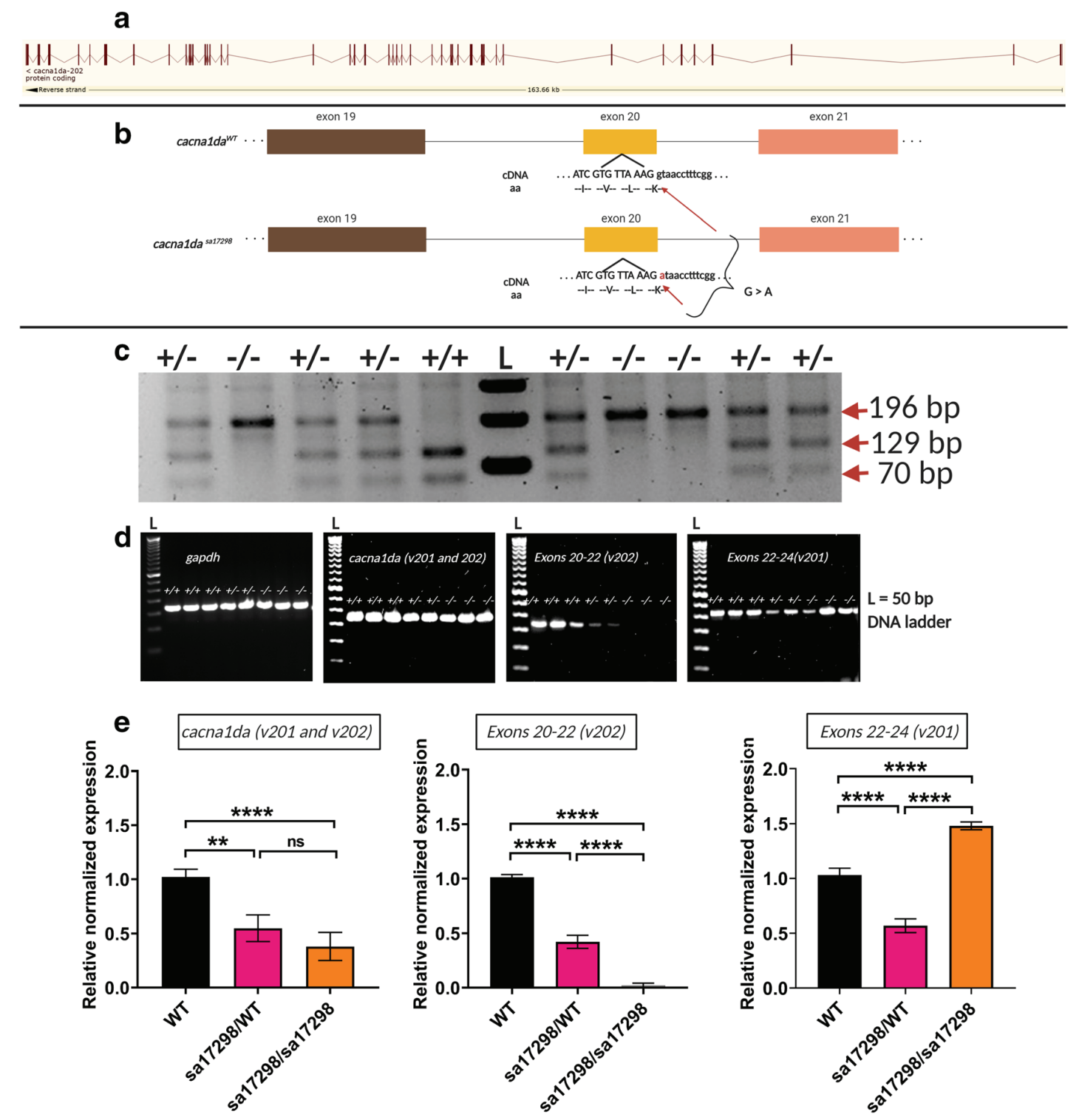

Fig. 1 Zebrafish cacnalda mutant allele (sa17298) and its molecular consequence. a Schematic representation of the zebrafish cacnalda transcript. Red bars represent exons and lines represent introns. Image retrieved from ensembl.org. b Schematic representation of zebrafish cacnalda and sal7298 mutation. Nucleotides in upper case are within the exon while those in lower case are within the intron. c Sample gel electrophoresis of BstEII restriction digest and resulting PCR products to determine genotype of fish/larvae. BstEII does not cut homozygous

sa17298, but cuts WT into two bands (70 and $119 \mathrm{bp}$ ) and heterozygous sa17298 into three bands (70,119 and $196 \mathrm{bp})$. Note the multiple bands in the BstEII digested versus the undigested corresponding samples. L: 1 kilobase DNA ladder, +/+: cacnalda ${ }^{W T}$ WT + +-: cacnalda ${ }^{\text {sa17298/WT }},-/-$ : cacnalda ${ }^{\text {sal7298/sa17298 }}$. d cacnalda mRNA levels as measured by agarose gel electrophoresis (rt-PCR). e cacnalda mRNA levels as measured by qPCR. cacnalda mRNA expression is normalised against gapdh and $r p s 18$ 


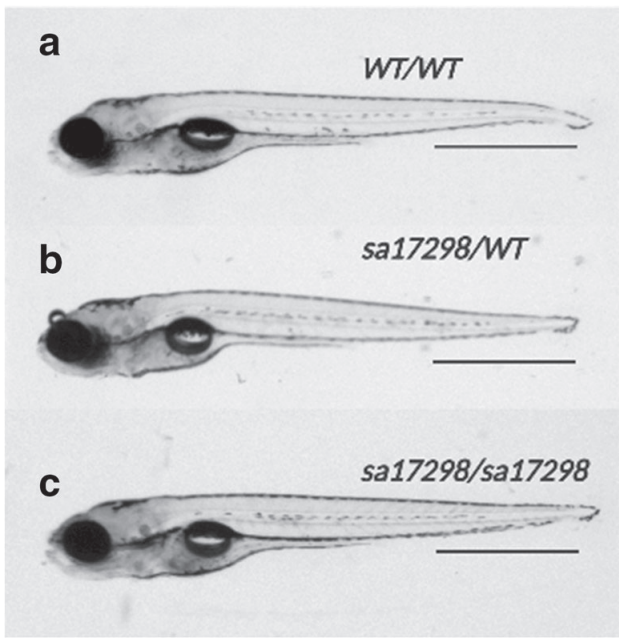

Fig. 2 Morphology of a WT cacnalda $a^{W T / W T}$ b heterozygous cacnalda ${ }^{\text {sa17298/WT }}$ and $\mathbf{c}$ homozygous cacnalda ${ }^{\text {sal7298/sa17298 }}$. Both heterozygous and homozygous mutants are morphologically indistinguishable from WT siblings. Scale bar $(\mathrm{a}-\mathbf{c})$ : $1 \mathrm{~mm}$

variant 201 were used, homozygous mutants showed a 50\% increase, while heterozygous mutants showed a $50 \%$ reduction in levels of variant 201 (Fig. 1e).

\section{Morphological Analysis of sa17298 Mutants}

When AB-outcrossed mutants were bred, the resulting heterozygous and homozygous mutants were morphologically indistinguishable from their WT siblings (Fig. 2). All experiments were carried out using the $\mathrm{AB}$ outcrossed fish. ${ }^{1}$ Based on genotyping results, less than 5\% of homozygous sa17298 larvae survived until adulthood. No impairment in the touchevoked response of heterozygous and homozygous sa17298 larvae was observed when compared to WT (data not shown).

\section{Acoustic Startle Response and Prepulse Inhibition}

Four different auditory stimuli $(40,50,60,70 \mathrm{~dB})$ at $660 \mathrm{~Hz}$ were tested to determine the suitable startle stimuli, and it was determined that $70 \mathrm{~dB}$ evoked the strongest startle response at $p<0.05$ (Fig. 3a). When a $50-\mathrm{dB}$ prepulse preceded the $70-\mathrm{dB}$ stimulus, larval startle response was decreased (Fig. 3b). Oneway ANOVA revealed no statistically significant difference in the PPI (\%) between WT and either mutant genotype (i.e. heterozygotes and homozygotes) (Fig. 3c) $[F(2,16)=$

\footnotetext{
${ }^{1}$ It is important to note that gross morphological defects such as craniofacial abnormalities, smaller, fused or absent eyes, curved body axis, yolk sac necrosis and hyperpigmentation were visible as early as $2 \mathrm{dpf}$ in a proportion of the offspring $(\sim 25 \%)$ when male and female adult heterozygous $s a 17298$ (obtained directly from ZIRC) were in-crossed. These morphological defects were present in heterozygous and homozygous sa17298 populations as well as WT larvae (Suppl. Fig. 2). We therefore recommend that the line be outcrossed over multiple generations to remove other mutations that may confound the interpretation of results.
}

$1.403, p=0.2745]$. Notably, however, there was a tendency of homozygotes towards statistical significance (i.e. increased PPI).

\section{Locomotor Assessment of sa17298 Larvae}

To determine if the sa17298 mutation modulates locomotor behaviour, one batch of larvae were tracked in dark conditions ( $0 \%$ light) while another group of larvae were tracked in $100 \%$ light. Mann Whitney $U$ test showed no statistically significant difference between the genotypes $\left[\right.$ cacnalda ${ }^{W T / W T}(\mathrm{Mdn}=$

a

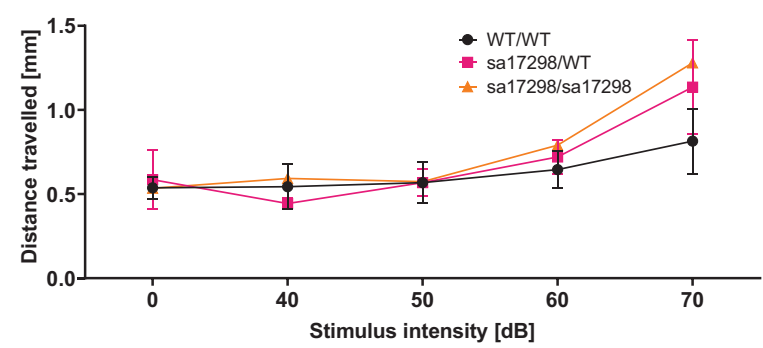

b

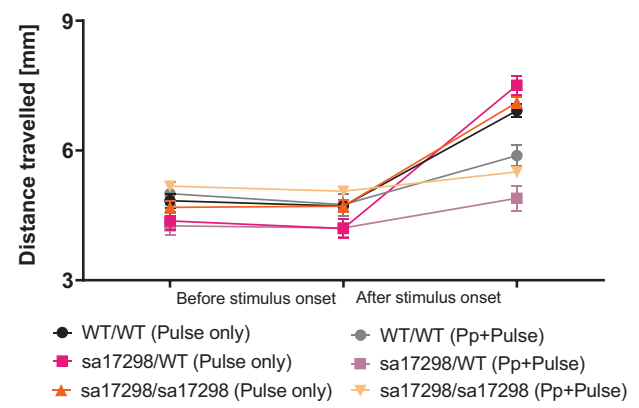

C

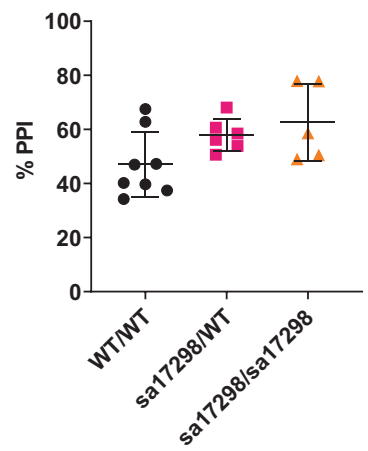

Fig. 3 Acoustic startle response and PPI. a Graph of distance travelled when presented with different auditory stimuli. Data analysed using twoway ANOVA and presented as mean \pm SEM. b Preceding a startle with a prepulse stimulus decreases the startle response of larvae. Data shown as mean \pm SEM. c \% PPI of larvae. One-way ANOVA revealed no overall significant difference among the groups. Tukey's multiple comparison post hoc test however showed a strong tendency of homozygous mutant larvae towards significance (i.e. enhanced PPI). cacnalda ${ }^{W T W T}(N=8)$, cacnalda ${ }^{\text {sal }}$ (298/WT $(N=6)$ and cacnaldd ${ }^{\text {sal7298sal } 17298}(N=5), p>0.05$. Error bars represent mean \pm SD. ASR, acoustic startle response; Pp, prepulse; PPI, prepulse inhibition. cacnalda $a^{W T / W T}$ vs cacnalda $a^{\text {sa17298/WT }}: p=0.2002$ cacnalda $^{W T W T}$ vs cacnald $a^{\text {sal } 7298 / s a 17298}: p=0.0646$, cacnald $a^{\text {sal }^{2} 298,}$ ${ }_{W T}$ vs cacnalda ${ }^{\text {sal } 7298 \text { sal } 7298}: p=0.7702$ 


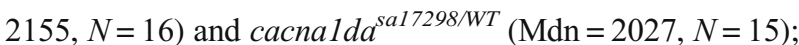
$U=109, p=0.6823]$ when tracked in the dark (Fig. 4a). On the other hand, when larvae where tracked in $100 \%$ light conditions, Mann Whitney $U$ test indicated that the total distance travelled by cacnalda ${ }^{W T W T}(\mathrm{Mdn}=1632, N=21)$ was significantly lower than cacnalda ${ }^{\text {sal7298/WT }}(\mathrm{Mdn}=2039, N=22)$; $U=92, p=0.0005$ ] (Fig. 4b).

\section{Larval Behaviour in Response to Abrupt Change in Illumination}

The locomotor behaviour of larval zebrafish differs depending on light-dark transitions [47]. Based on the observations made when two groups of larvae were independently tracked in either light or dark conditions, we hypothesised that changes in illumination in the tracking chamber would alter locomotor activity of sal 17298 larvae. We analysed spontaneous swimming after a 15 -min acclimation period. Swimming in each illumination state (light-dark) was recorded for $10 \mathrm{~min}$ (Fig. 5).

In Fig. 5 a, larvae demonstrated an increased locomotor activity when transitioned from light to dark shown by a non-RM two-way ANOVA analysis [genotype $F(1,28)=$ 9.645, $p<0.01$; illumination $F(1,28)=162.3, p<0.0001$; interaction $F(1,28)=3.241, p=0.0826]$. Tukey's post hoc test revealed that both cacnalda $a^{W T / W T}(p<0.0001)$ and cacnalda ${ }^{\text {sa17298/WT }}(p<0.0001)$ travelled higher distances in the dark than in the light. Additionally, the post hoc test indicated statistical significance between cacnalda $a^{W T / W T}$ and cacnalda ${ }^{\text {sa17298/WT }}$ when they transitioned to the dark $(p<0.01)$, whereas Student's $t$ test showed statistical significant difference between cacnalda ${ }^{W T W T}$ and cacnalda $a^{\text {sal7298 }}$ ${ }^{W T}$ in the light phase $(p<0.05)$.

A previous study reported that changes in illumination altered the zone preference of larvae in the thigmotaxis test [42].
Therefore, we sought to determine if changes in illumination modulated the zone preference of cacnalda $a^{W T / W T}$ and cacnalda $^{\text {sal7298/WT }}$ larvae. Larvae were assessed for the distance travelled (\%) in the outer zones of a 24-well plate arena within $10 \mathrm{~min}$.

As seen in Fig. 5b, there was no significant difference in the zone preference of cacnalda ${ }^{W T / W T}$ and cacnalda $a^{\text {sa17298/WT }}$ larvae using non-RM two-way ANOVA analysis in the light-dark transition [genotype $F(1,28)=0.1310, p=0.7201$; illumination $F(1,28)=$ $0.4996, p=0.4855$; interaction $F(1,28)=0.01618, p=$ $0.8997]$

\section{Assessment of Startle Response to Repetitive Dark Flashes}

Zebrafish larvae respond to startle stimuli such as unexpected light or sound stimulus and quantifying changes in distance travelled have been established as a suitable readout of startle response [43, 48]. Following the "startle response to a dark flash" protocol of Norton, larvae were presented with 30 dark flashes repeated every $3 \mathrm{~s}$ [43].

The locomotor activity of the last $21 \mathrm{~s}$ of the 10-min basal locomotor activity was measured preceding the dark flashes that were every $3 \mathrm{~s}$ thirty times (Fig. 6a). In general, cacnalda $^{W T / W T}$ and cacnalda ${ }^{\text {sal7298/WT }}$ larvae neither swum the same distance during 21 -s basal locomotor activity [ $t$ $(41)=3.879, p=0.0004$, Fig. 6 a] nor the 90 -s startle experiment $[t(41)=2.627, p=0.0120$, Fig. 6b]

The distance moved in response to the first and second dark flash was greater than the distance moved in response to subsequent stimuli. Whereas WT larvae showed signs of habituation after the 2 nd stimulus, habituation was only visible after the 19th stimulus in heterozygous mutants. Multiple $t$ test analysis showed that at several time points, cacnald $a^{\text {sa17298 }}$
Fig. 4 Locomotor activity of WT and heterozygous sa17298 zebrafish larvae at $6 \mathrm{dpf}$, tracked independently under different illumination conditions. a Tracking in the dark reveals no difference in locomotor activity between cacnalda $a^{W T W T}(N=16)$ and cacnalda $a^{\text {sa17298/WT }}(N=15)$. b Tracking in $100 \%$ light reveals a statistically significant difference in locomotor activity between cacnalda ${ }^{W T / W T}(N=21)$ and cacnalda ${ }^{\text {sal } 7298 / W T}(N=22)$. $* * * p<0.001$. Data analysed using Mann Whitney $U$ test and represented as mean $\pm \mathrm{SD}$
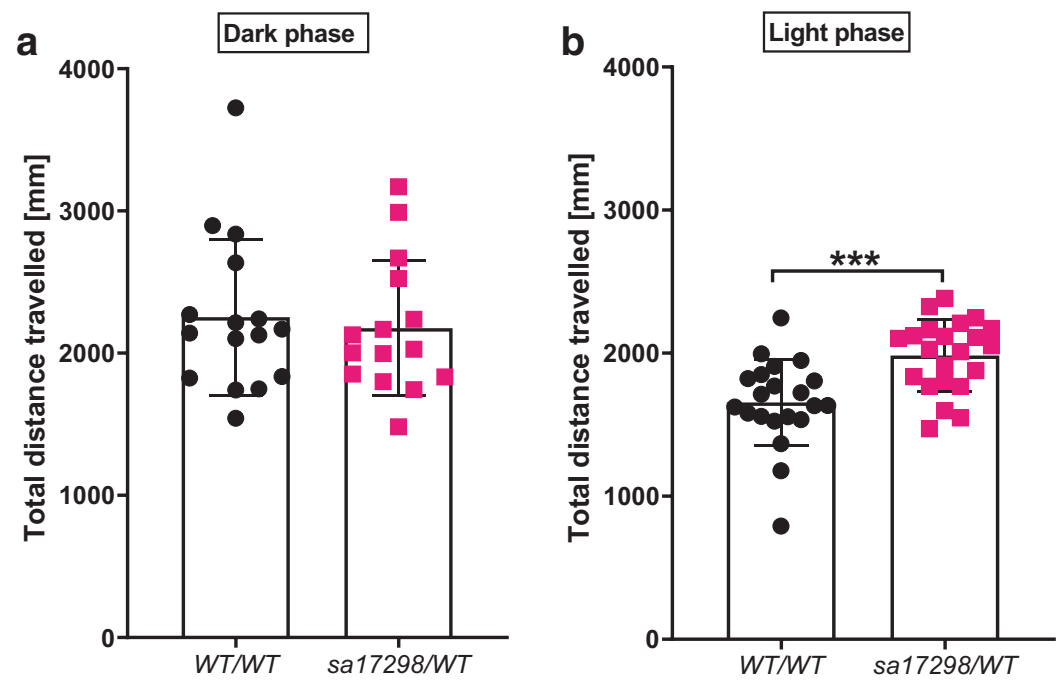
Mol Neurobiol

${ }^{W T}$ larvae were significantly more active than cacnalda $a^{W T W T}$ at $p<0.05$. Overall, cacnalda $a^{W T N T}$ displayed a robust startle response relative to baseline than cacnalda $a^{\text {sa17298/WT }}$ [i.e. when we compared the last $21 \mathrm{~s}$ prior to stimulus onset with startle stimuli response] (Fig. 6c).

\section{Effects of Neuroactive Drugs on the Locomotor Activity of WT and Heterozygous sa17298 Zebrafish Larvae}

In zebrafish, hyperlocomotion is often characterised as a phenotype of epilepsy- [45, 49-52], anxiety- [51, 53] or psychosis-like behaviour [51, 54, 55]. To understand the possible cause of the hyperlocomotor behaviour, we observed in heterozygous sa17298/WT larvae, three neuroactive drugs i.e. RISP, HALO and VPA were evaluated for their ability to modulate the larval hyperactivity after 30 min of tracking. Non-RM two-way ANOVA revealed a significant effect of both genotype $[F(1,56)=8.010, p=0.0064]$ and treatment $[F(1,56)=5.799, p=0.0193]$ on locomotor activity when larvae were exposed to $5 \mu \mathrm{M}$ RISP for $2 \mathrm{~h}$. No significant interaction $[F(1,56)=1.066, p=0.3064]$ was observed. However, a Tukey's post hoc test showed that RISP was only effective in decreasing the locomotor activity of $s a 17298 / W T(p<0.05)$ but not WT/WT $(p>0.05)$ when compared with their untreated control (Fig. 7a).

To ascertain whether a prolonged exposure of zebrafish larvae to RISP would enhance RISP's effectiveness in affecting locomotor activity, a set of larvae were incubated in RISP for $24 \mathrm{~h}$ and the locomotor activity measured subsequently. As seen in Fig. 7b, non-RM two-way ANOVA showed an overall statistically significant effect of RISP on larval locomotor activity after 24-h pre-exposure [genotype $[F(1,36)=$ $10.77, p=0.0023]$; treatment $[F(1,36)=51.41, p<0.0001]$; interaction $[F(1,36)=1.351, p=0.2527]]$. Tukey's post hoc test revealed a statistically significant decrease in locomotor activity in larvae treated with $5 \mu \mathrm{M}$ RISP when compared with their untreated control at least $p<0.01$ such that baseline locomotor activity became comparable between $5 \mu \mathrm{M}$ [WT/ WT vs sal 7298/WT] at $p=0.4262$.

Furthermore, two-way non-RM ANOVA of larvae exposed to $50 \mu \mathrm{M}$ HALO for $2 \mathrm{~h}$ (Fig. 7c) yielded a statistically significant effect of genotype $[F(1,45)=15.03, p=0.0003]$ and treatment $[F(1,45)=78.55, p<0.0001]$ on locomotor activity with no significant interaction $[F(1,45)=1.518, p=$ 0.2243]. Subsequent Tukey's post hoc analysis revealed a reduction in locomotor activity of the $50 \mu \mathrm{M}$ treated larvae when compared to their respective within group control at least $p<0.001$. There was no statistical significance observed between $50 \mu \mathrm{M}$ [WT/WT vs sa17298/WT] at $p=0.3117$.

In Fig. 7d, larvae pre-treated in VPA for $2 \mathrm{~h}$ displayed an overall significant effect of genotype $[F(1,60)=33.15$, $p<0.0001]$ and treatment $[F(1,60)=23.57, p<0.0001]$ on
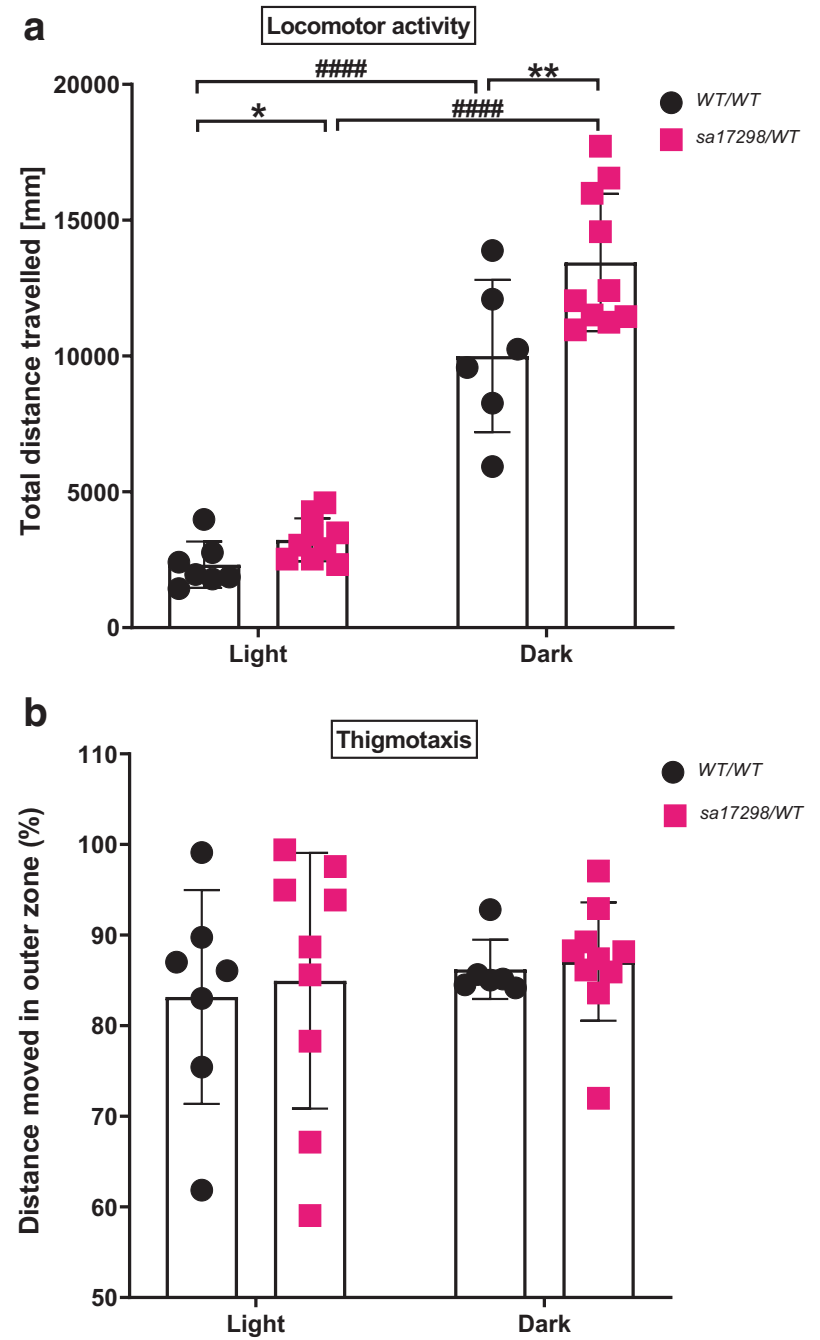

Fig. 5 Behaviour of WT and heterozygous sal 7298 zebrafish larvae at $6 \mathrm{dpf}$ in the light-dark transition test. Each dot represents an individual larval measurement. Data analysed using non-RM two-way ANOVA followed by Tukey's post hoc test. Data represented as mean \pm SD. a Light-dark transition elicits an increase in locomotor activity in both genotypes with locomotor difference between cacnalda ${ }^{W T / W T}$ and cacnalda $^{\text {sa17298/WT }}$ reaching statistical significance in both illumination

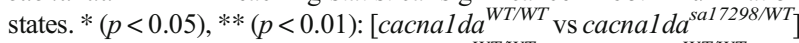
in the dark, \#\#\#\# (p<0.0001): [cacnalda $a^{W T / W T}$ vs cacnalda $\left.{ }^{W T / W T}\right]$ and [cacnalda ${ }^{\text {sa17298/WT }}$ vs cacnalda ${ }^{\text {sa17298/WT }}$ ]. b Zone preference of WT and heterozygous sa17298 larvae (thigmotaxis) represented as \% total distance moved in the outer zone in the light-dark transition test

larval locomotor activity with no significant interaction $[F$ $(1,60)=0.1149, p=0.7358]$ observed when a two-way nonRM ANOVA was performed. Tukey's post hoc analysis showed statistically significant effect of VPA to decrease larval locomotor activity across groups at least $p<0.01$ when compared with untreated larvae of the same genotype. However, VPA was unable to reduce the hyperlocomotion of $s a 17298 / W T$ larvae to a comparable level as the VPAtreated WT/WT group $(p=0.0023)$. 


\section{Effects of Neuroactive Drugs on the Behaviour of WT and Heterozygous sa17298 Zebrafish Larvae in the Light-Dark Test}

The hyperlocomotor activity of heterozygous larvae relative to WT in the light and dark states was reversed after the exposure of larvae to all the treatment options i.e. 24-h exposure in $5 \mu \mathrm{M}$ RISP and 2-h exposure in $5 \mu \mathrm{M}$ RISP, $50 \mu \mathrm{M}$ HALO and $100 \mu \mathrm{M}$ VPA at $p>0.05$. Larvae in either genotypes exposed to all the treatment groups but HALO were capable of eliciting a light-dark response by increasing their locomotion $(p<0.001)$ (See Fig. 8 and Suppl. Table 2). a

a Before onset of startle stimuli

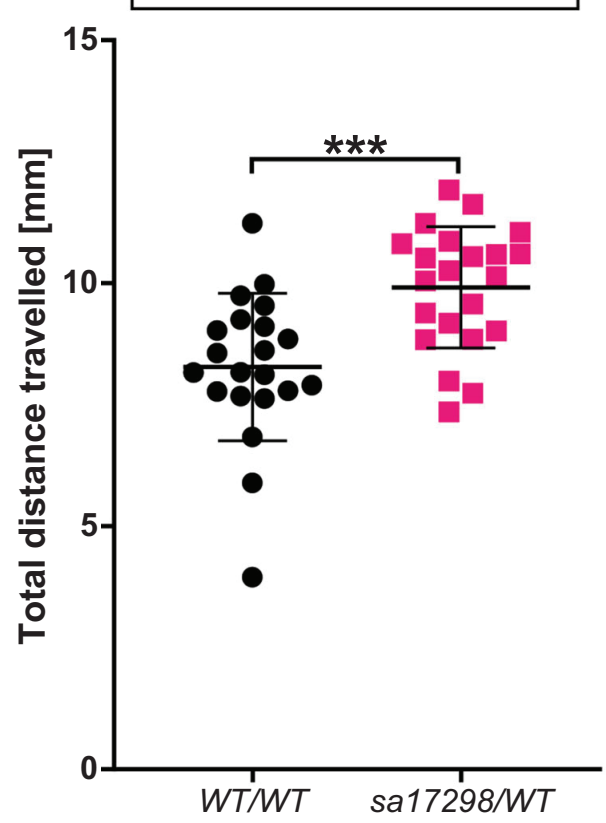

b

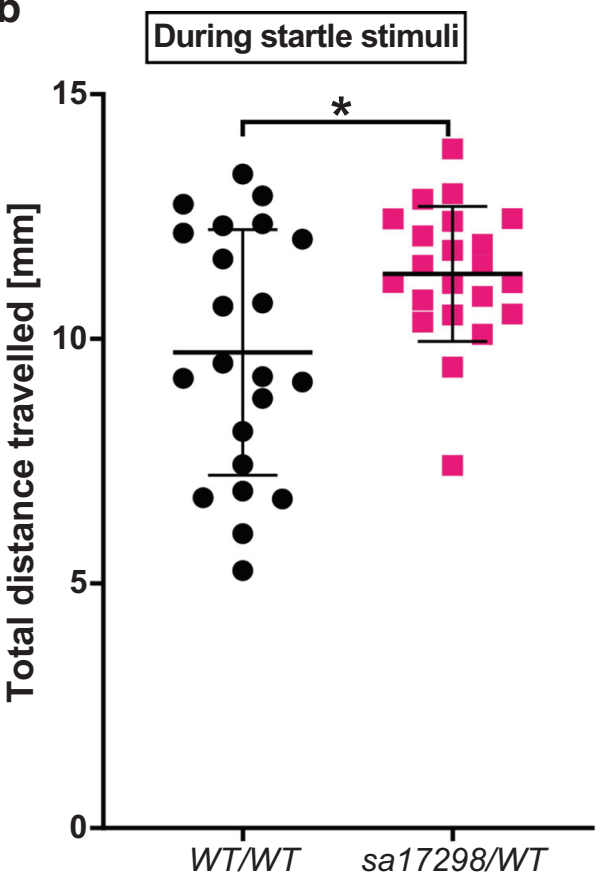

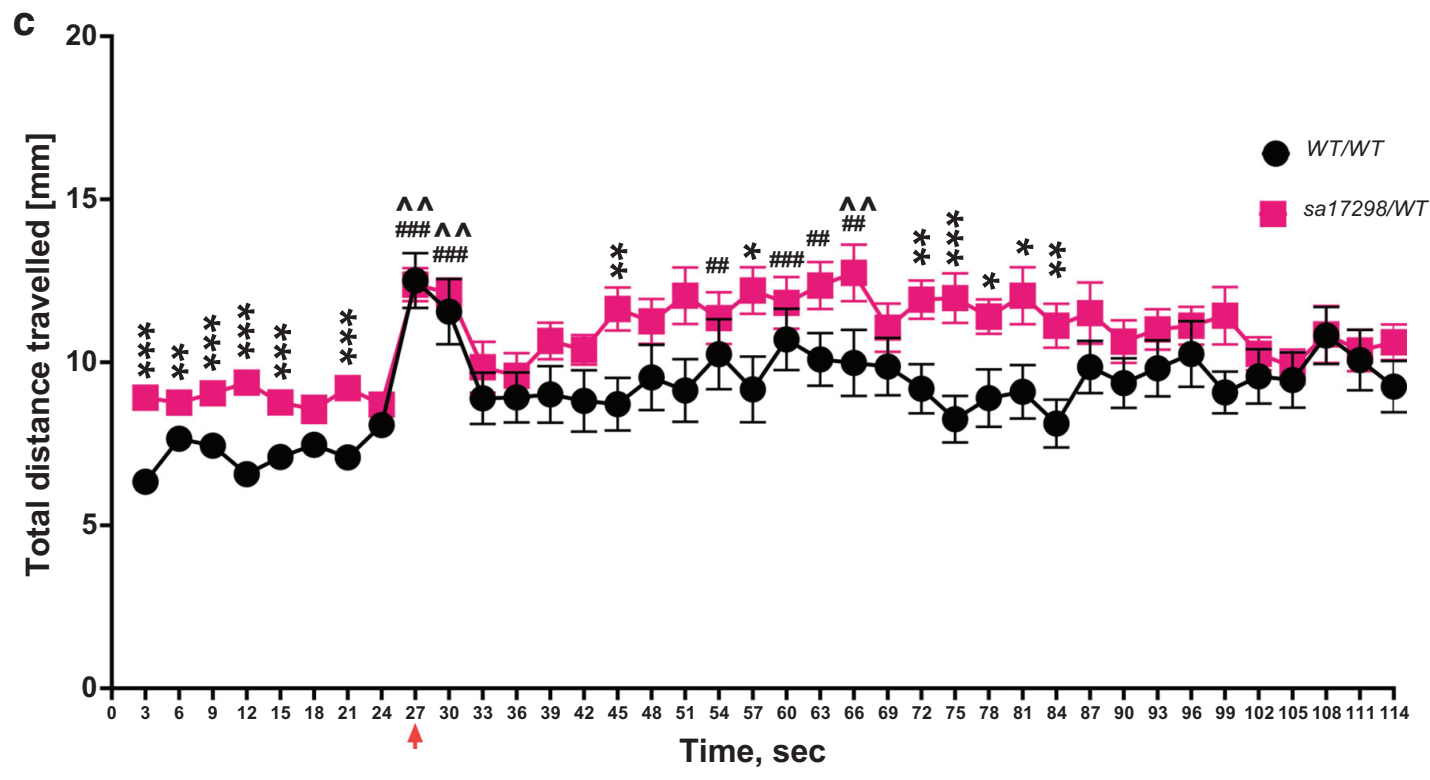

Fig. 6 Startle response to dark flashes. a Baseline locomotor activity $21 \mathrm{~s}$ prior to onset of startle stimuli, $* * * p<0.001$. Data represented as mean \pm SD. b Mean distance moved in response to startle stimuli. Unpaired Student's $t$ test showed cacnalda ${ }^{W T / W T}$ moved less than cacnalda ${ }^{\text {sa17298/WT }}$ during the 90 -s startle stimulation, $* p<0.05$. Data represented as mean \pm SD. $\mathbf{c}$ Distance moved in response to startle stimuli represented as mean \pm SEM. Paired Student's $t$ test analysis between cacnalda ${ }^{W T W T}$ and cacnalda $a^{\text {sa17298/WT }}$ with statistical significance represented as $* p<0.05,{ }^{\#} p<0.01,{ }^{\wedge} p<0.001$. Red arrow: onset of startle stimulus (dark flashes) 

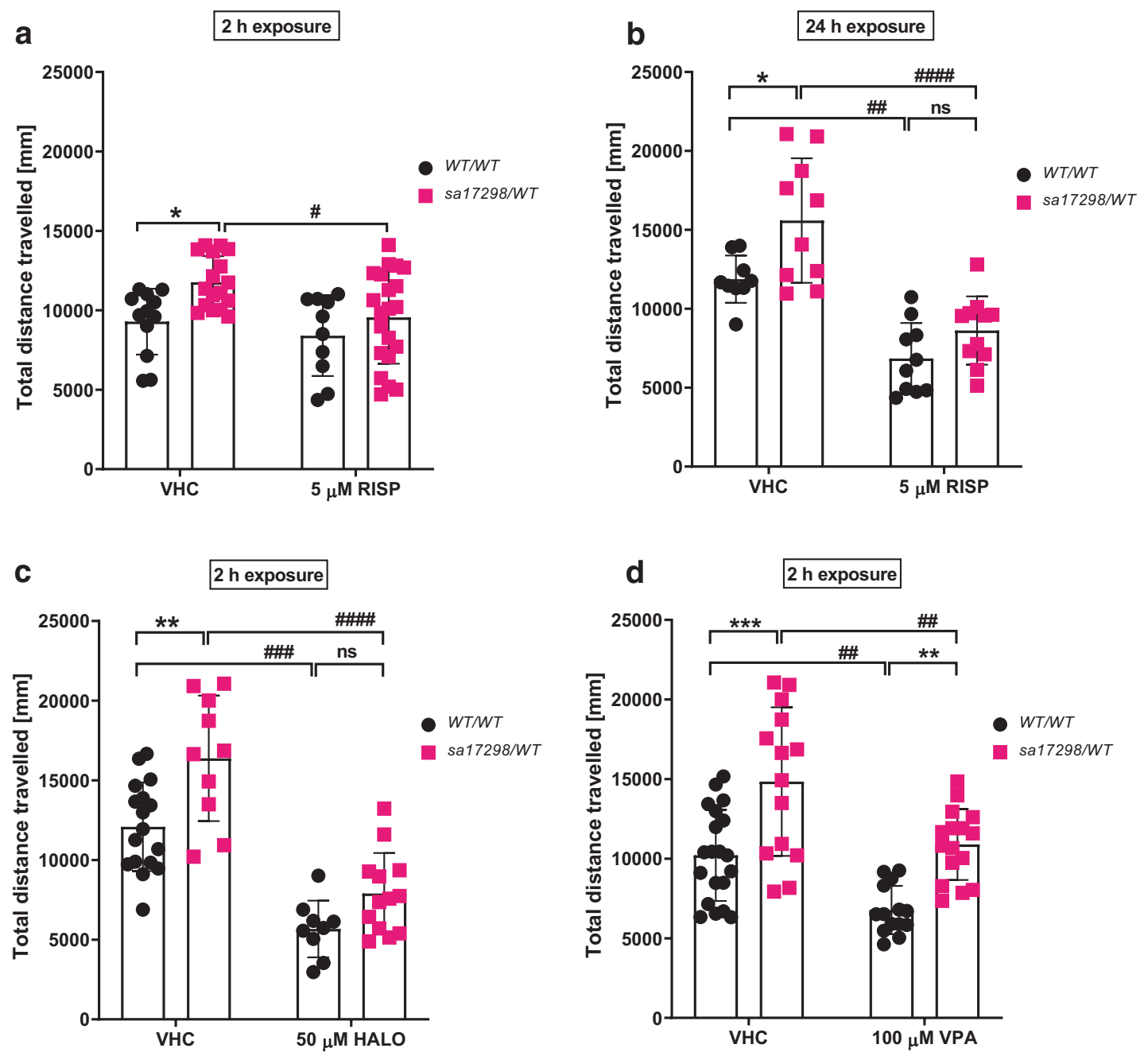

Fig. 7 Effects of neuroactive drugs on the locomotor activity of 6-dpf WT and heterozygous sa17298 larvae. Larvae were exposed to different neuroactive drugs. Each dot represents individual larval measurement. Data analysed using non-RM two-way ANOVA followed by Tukey's post hoc test. Data represented as mean \pm SD. a 2-h RISP, b

\section{Effects of Neuroactive Drugs on the Behaviour of WT and Heterozygous sa17298 Zebrafish Larvae in the Startle Response to Dark Flashes}

Larvae treated in vehicle control behaved similarly as their untreated (medium) counterparts previously described. In the 2-h exposure to RISP group, there was no difference in locomotor activity between WT and heterozygous mutant larvae prior to the onset of the dark flashes. After dark flashes onset, there was reduced startle response of heterozygous larvae in the first five dark stimuli with the next seven dark flashes resulting in comparable behaviour to the untreated group. In general, RISP at $2 \mathrm{~h}$ reduced heterozygous larval reactivity to the startle inducing dark flashes ( $p>0.05$, Fig. 9a). At 24-h exposure, RISP reversed the hyperlocomotor activity of heterozygous larvae prior to the onset of dark stimuli $(p>0.05$,

24-h RISP c 2-h HALO and d 2-h VPA. HALO, haloperidol; RISP, risperidone; VPA, valproic acid. $* p<0.05, * * p<0.01, * * * p<0.001$ $\left[\right.$ cacnalda ${ }^{W T / W T}$ vs cacnalda ${ }^{\text {sal7298/WT }}$ ] in respective groups. ${ }^{\#} p<0.05$, ${ }^{\# \#} p<0.01,{ }^{\# \#} p<0.001,{ }^{\# \# \#} p<0.0001$ [cacnalda ${ }^{W T / W T}$ vs cacnalda ${ }^{W T}$ WT $]$ and [cacnalda ${ }^{\text {sal } 17298 / W T}$ vs cacnalda $\left.{ }^{\text {sa17298/WT }}\right]$

Fig. 9b). However, upon onset of dark flashes, heterozygous larvae behaved similar to their untreated siblings i.e. hyperactivity ( $p<0.01$, Fig. 9b). On the other hand, HALO was able to reverse the hyperlocomotor activity of heterozygous larvae both prior to and at the presentation of the startle inducing dark flashes $(p>0.05$, Fig. 9c). Whereas, VPA treatment resulted in a delayed response of larvae to dark flashes and an overall reversal of hyperactivity of the heterozygous larvae ( $p>0.05$, Fig. 9d) (For modulatory activity of all drugs over time, see Suppl. Fig. 3 and Table 3).

\section{EEG Assessment}

Student's $t$ test analysis of tectal EEG recordings of 6-dpf larvae indicated that there were no statistically significant differences with regard to the number of epileptiform-like 
discharges between cacnalda ${ }^{W T / W T}(M=2.088, N=12)$ and cacnald ${ }^{\text {sal } 7298 / W T}(M=4.333, N=15) ; t(25)=0.8741, p=$ 0.3904 (for example, see Suppl. Fig. 4).

\section{Discussion}

In this study, we describe for the first time neurobehavioural changes in larval zebrafish with an essential splice variant mutation (sa17298) in the cacnalda gene. Since our mutant (sa17298) harbours a single point mutation $(\mathrm{G}>\mathrm{A})$ at the donor splice site of intron 20-21 of the cacnalda-202 transcript variant, we anticipated that this could lead to
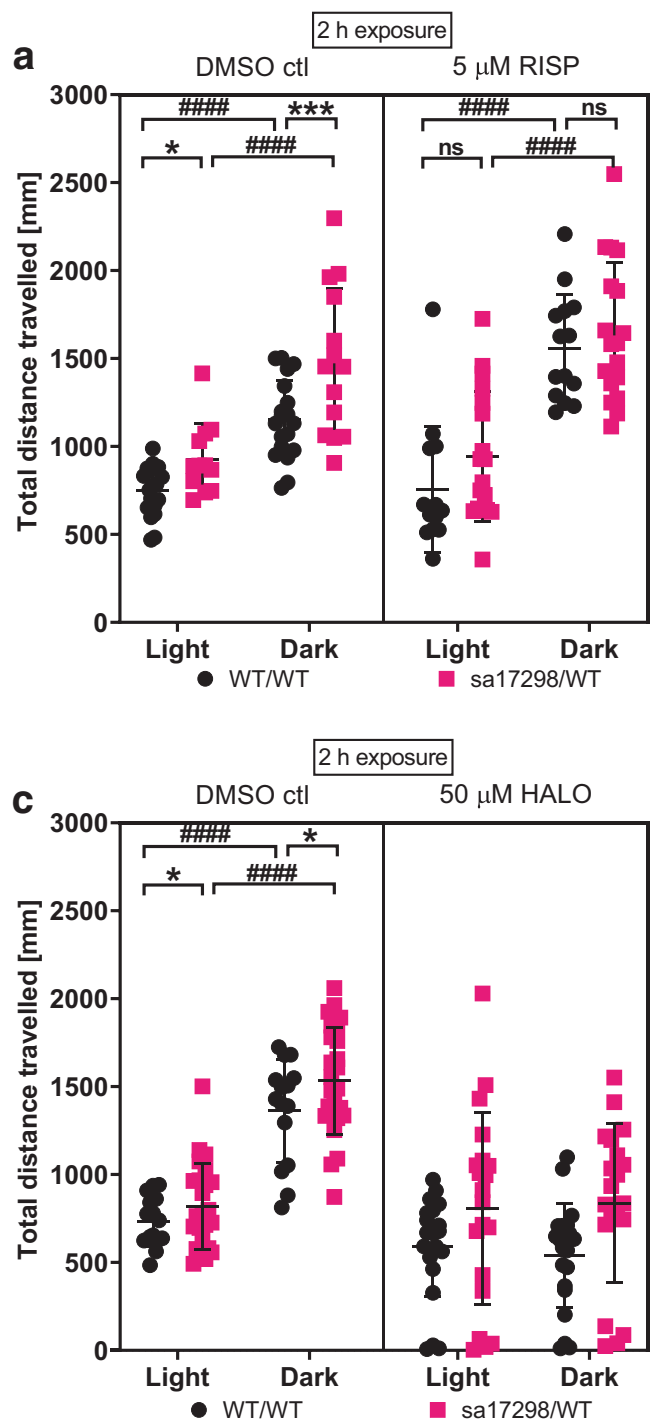

Fig. 8 Effects of neuroactive drugs on the behaviour of 6-dpf WT and heterozygous sa17298 larvae in the light-dark test. Larvae were exposed to different neuroactive drugs. Each dot represents individual larval measurement. Data analysed using two-way ANOVA followed by multiple comparison $t$ test. Data represented as mean \pm SD. a 2-h RISP, b 24-h dysregulation of cacnalda mRNA or protein expression, thus mimicking classical GOF or loss-of-function mutations. Agarose gel electrophoresis of rt-PCR products did not suggest exon skipping as a consequence of the mutation. The results showed significantly reduced "overall" cacnalda mRNA and 202 transcript levels in both heterozygous and homozygous mutants relative to WT. For the 201 transcript, while heterozygous mutants showed reduced levels, homozygous mutants on the contrary showed increased levels. We speculate that the $s a 17298$ splice variant mutation leads to a premature termination codon in variant 202 , as a result of intron retention, thereby resulting in nonsense-mediated decay, thus resulting in loss or significant reduction of this
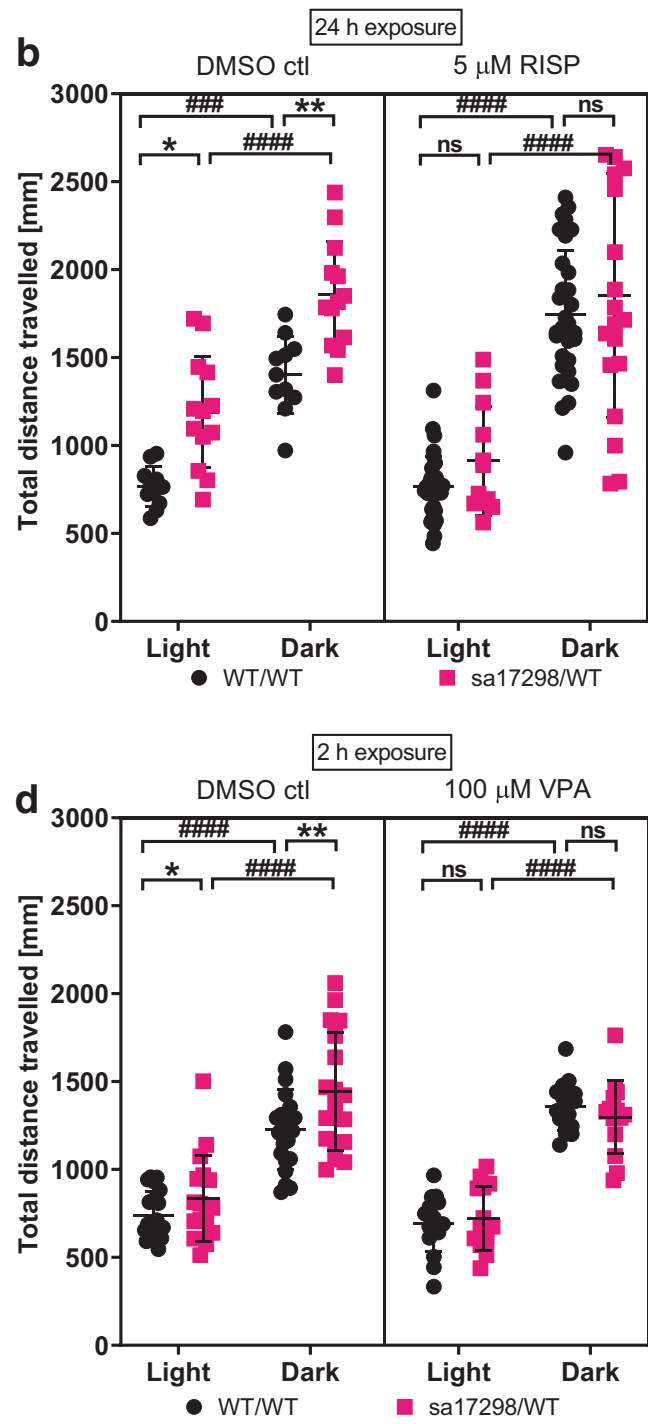

RISP, c 2 h HALO and d 2-h VPA. ctl, control; HALO, haloperidol; RISP, risperidone; VPA, valproic acid. $* p<0.05$, ** $p<0.01$, *** $p<0.001$ [cacnalda ${ }^{W T / W T}$ vs cacnalda ${ }^{\text {sal } 17298 / W T}$ ] in respective groups. ${ }^{\# \# \#} p<0.001,{ }^{\# \# \#} p<0.0001$ [cacnalda $a^{W T / W T}$ vs cacnalda ${ }^{W T}$ groups. ${ }_{W T} p<0.001,{ }^{2} p<0.0001[$ cacnalda
[cacnalda 
particular transcript and subsequent compensation through an increase in levels of transcript variant 201.

Our study revealed significant behavioural impairments in cacnalda ${ }^{\text {sa17298/WT }}$ mutants when assayed for locomotor activity (under light conditions), during light-dark transition and in the startle response to dark flashes. However, we did not observe significant PPI deficits, thigmotaxis-related abnormalities or epileptiform-like discharges in larval brains as measured by EEG recordings.

The behavioural response of cacnalda $a^{W T W T}$ in the lightdark stimulus task is in agreement with other studies [41,
56-59], which showed an abrupt increase in locomotor activity of larvae during rapid switching from light to dark. Kedra et al. [56] revealed that homozygous $t s c 2$-deficient larvae (model of tuberous sclerosis complex, in which $90 \%$ of patients display seizures) exhibited the same behavioural response as described here for cacnalda $a^{\text {sal7298/WT }}$ mutants i.e. a pronounced increase in locomotor activity of $t s c 2^{-/-}$larvae compared with their control counterparts in the dark phase of the light-dark stimulus task. $t s c 2^{-/}$larvae also displayed increased thigmotaxis as well as a preference to light phase in the light-dark preference task. Exacerbation of thigmotactic
Fig. 9 Time series graph of the effects of neuroactive drugs on the behaviour of 6-dpf WT and heterozygous sa17298 larvae in the startle response to dark flashes test. Larvae were exposed to different neuroactive drugs. Each dot represents individual larval measurement. Data represented as mean \pm SEM. a 2-h RISP, b 24-h RISP, c 2-h HALO, d 2-h VPA. HALO, haloperidol; RISP, risperidone; VPA, valproic acid
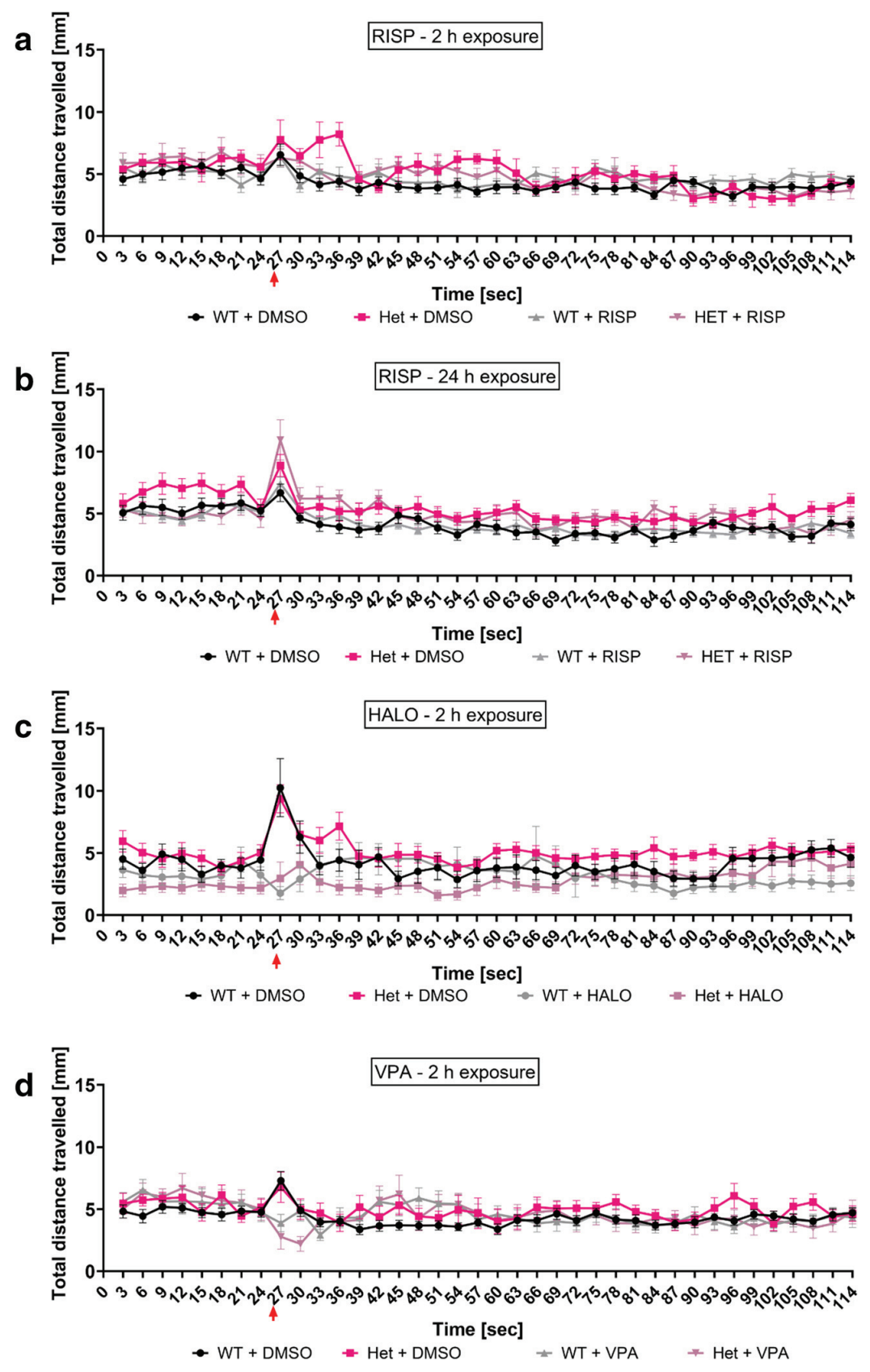
behaviour in zebrafish during dark phase is regarded as a marker of anxiety-like behaviour [42, 60], thus indicating an anxiety-like phenotype for $t s c 2^{-/-}$larvae. In contrast, we did not observe changes in thigmotaxis between cacnalda genotypes. Thus, it is unlikely that the increased locomotor activity of cacnalda ${ }^{\text {sal } 7298 / W T}$ larvae in the dark phase of the lightdark stimulus task was a result of cacna lda-mediated anxietylike behaviour.

Increased locomotor activity, as a readout of tonic-cloniclike seizures, was previously described in zebrafish genetic models of epilepsy [49, 61, 62], which correlated well with the occurrence of epileptiform-like discharges in larval brains as measured by EEG. In some human CACNA1D mutation carriers, different types of seizures have been observed (focal and/or generalised seizures) $[17,20,63]$. In our study, we observed epileptiform-like discharges in the EEG assay only in 3 out of 15 cacnalda $a^{\text {sal } 7298 / W T}$ larvae. Thus, it is unlikely that the hyperlocomotion displayed by cacnalda $a^{\text {sa17298/WT }}$ mutants is associated with seizures.

CACNA1D mutations have been implicated earlier in SCZ $[8,13,21]$. In behavioural pharmacology in rodents, the most common approach is to mimic psychotic-like positive symptoms by administering drugs that target N-methyl-D-aspartate (NMDA) (antagonism) or dopaminergic (agonism) receptors. These drugs induce psychosis in rodents with hyperactivity as a readout of this symptom (for review, see [64]). Interestingly, pharmacological studies revealed high correlation with regard to the ability of larval and adult zebrafish to mimic positive SCZ symptoms. For example, ketamine [65, 66] and MK-801 (also called dizocilpine) [54, 67] induce a robust increase in locomotion. Moreover, d-amphetamine or $\mathrm{D}_{2}$ receptor agonists elicit the same locomotor response in adult zebrafish $[68,69]$ as in rodents [70-74]. Previously, hyperlocomotion was observed in mouse genetic models of SCZ with mutations in Discl [75] and Nrg1 [76]. In larval zebrafish, this feature was also commonly observed in mutants harbouring mutations in different genes indicated by GWAS as SCZ susceptibility genes [77]. A study by Thyme et al. [77], which generated and analysed 132 different mutants of SCZ-risk genes, showed that hyperlocomotion was sometimes observed in a number of these fish.

To obtain further insight into the neurobehavioural changes in cacnalda ${ }^{\text {sa17298/WT }}$ larvae, we evaluated sensorimotor gating and habituation in larvae by performing two tests - i.e. ASR and PPI tests, as well as the startle response to dark flashes assay. The startle response to dark flashes assay, in which animals habituate to dark flashes when exposed to continuous light stimulus, allows for the assessment of nonassociative memory [43, 48, 78, 79], which is also impaired in most schizophrenic patients [80, 81]. The ASR and PPI are the most commonly used tasks in SCZ research with an altered PPI response observed in schizophrenia patients [82-85], rodent [86-88] and some zebrafish models of SCZ [77]. The difference between PPI and startle response to dark flashes is the type of stimulus-i.e. auditory versus visual. As CACNA1D mutations have been previously implicated in deafness in humans [89, 90], mice [24] and even zebrafish [34], we assessed the hearing sensitivities of larvae across genotypes using different stimulus intensities (sound) and found them to be comparable. Nevertheless, no difference in PPI between WT and heterozygous mutants was observed, albeit with tendency of homozygotes towards enhanced PPI. The very low survival rate of homozygotes proved prohibitive with regard to our ability to collect and assay a sufficiently large set of larvae to reach statistical significance in our experimental setup. To the best of our knowledge, there is a lack of data as to whether CACNA1D mutation carriers are blind or have other visual deficits. However, a study reported Cacnald knockout mice to have slight visual impairment but found no evidence that this affected their behaviour in the Morris water maze test (a "visual" spatial learning and memory task) [25]. In the zebrafish, in situ hybridisation analysis revealed expression of cav1.3a mRNA in the eye (although faint) and ear, while cav1.3b expression was evident in the otic vesicle of 5dpf larvae. qPCR analysis indicated expression of cav1.3a mRNA both in the eyes and ears of adult zebrafish [27]. Taking into account that interlarval behavioural patterns were consistent throughout all experiments, we postulate that mutant visual acuity is preserved and that the behavioural phenotypes observed are a result of changes in brain activity and not visual disturbances. Although it appears that the cacnalda $a^{\text {sa17298/WT }}$ mutant response in this assay (startle response to dark flashes) might be related to cognitive symptoms of SCZ, this aspect of the mutant phenotype needs further investigation. Additionally, since the CACNA1D gene is duplicated in the zebrafish [27], the severity of the mutation may be attenuated through partial compensation by cacnaldb. Thus, this may explain the absence of PPI deficits in the mutants.

We performed pharmacological profiling of WT and cacnalda $^{\text {sal7298/WT }}$ larvae in the locomotor (under constant illumination), light-dark and startle response to dark flashes tests after exposure to different neuroactive drugs: two drugs used in the management of SCZ (HALO and RISP) and one drug (VPA) indicated for the treatment of epilepsy, and as add-on therapy in BP, migraine, anxiety or SCZ. The behavioural response of our WT fish after incubation with all 3 drugs is in agreement with previous studies which showed that all of them decreased locomotor activity in both larval and adult animals [35, 36, 54, 58, 91-93]. It seems that the response of WT larvae (i.e. hypoactivity) is a common feature for antipsychotics, as other drugs from this group also decreased baseline activity in WT larvae (e.g. droperidol, phenothiazine or clozapine) [94, 95]. With regard to VPA exposure, our study indicated that this drug substantially decreased activity of cacnalda $a^{\text {sa17298/WT }}$ larvae in all the three 
behavioural tests, although heterozygous mutants were significantly more active than their WT siblings in the locomotor test. The mechanism of action of VPA includes increasing levels of $\gamma$-aminobutyric acid (GABA) in the brain, inhibition of histone deacetylase and blockage of voltage-gated ion channels (sodium, potassium and calcium), such as $\mathrm{CaV}_{1.3}$ [96-98]. We therefore theorise that the effectiveness of VPA in decreasing locomotor activity in cacnalda mutants may act through a different mechanism other than seizure suppression.

In humans, CACNA1D mRNA has been detected in limbic and mesolimbic structures (i.e. amygdala, hippocampus, thalamus, hypothalamus, basal ganglia) [101, 102], which are enriched with dopaminergic neurons. Similarly, in mice, the Cacnald gene is expressed in limbic and striatal areas [103-105]. Sidi et al. [27] observed that the expression of cav1.3a is detected as early as 30 -h post-fertilisation (hpf) in the zebrafish brain (telencephalon, thalamic and hypothalamic diencephalon, midbrain, ventral hindbrain). From 48 hpf onwards, expression of cav1.3a becomes even more pronounced in almost the entire brain [27].

In conclusion, different zebrafish genetic models of epilepsy and to a lesser extent, SCZ, have emerged within the last 5 years (for comprehensive reviews see [106, 107]). Although the zebrafish brain is less complex than the mammalian one, fish are very sensitive to neuroactive compounds and their behavioural responses are easily tracked in 48-well plates. This allows for very rapid and efficient screening of compounds compared with rodents, thus enabling the identification of bioactive "hit" compounds for further investigation. Thus far, only zebrafish genetic models of epilepsy have been used successfully for discovering new therapeutic options in high-throughput screening assays [49, 108]. In SCZ research, there remains a need for identification of new antipsychotic agents with less side effects (especially those related to extrapyramidal symptoms) and effectiveness in alleviating negative and cognitive symptoms besides positive symptoms. Furthermore, with the advancements in genetic engineering methods in zebrafish [109-111], there is potential for using a precision medicine-based approach, where patient-specific mutations are introduced into zebrafish embryos to generate "customised" models for drug screening. This may aid scientists in tackling the pharmaco-resistance problem prevalent in SCZ patients, of which genetics plays a key role [see reviews [112, 113]. In relation to cacnalda $a^{\text {sa17298/WT }}$ larvae, our data appears promising, but further in-depth phenotyping is warranted to fully validate it as a new model of Cacnald-mediated SCZ.

Authors' Contributions NSB, CVE and KG conceptualised the experiments. All authors contributed to preparation of the manuscript. NSB and CVE revised and edited the final version. NSB carried out all larval morphological assessment, behavioural phenotyping and corresponding data analysis. LDW assisted with behavioural experiments. KG performed EEG experiments and analysis. NSB and KG prepared the figures. All authors read and approved the manuscript.

Funding Open Access funding provided by University of Oslo (incl Oslo University Hospital). This work was funded by the Research Council of Norway (ISP, BIOTEK2021/ DigiBrain) (for NSB and CVE) and partially supported by start-up funds from the Centre for Molecular Medicine Norway (for CVE). KG was funded by the European Union's Horizon 2020 research and innovation programme under the Marie SkłodowskaCurie (grant agreement No. 798703-GEMZ-H2020-MSCA-IF-2017).

Compliance with Ethical Standards All experiments were approved by the Norwegian Food Safety Authority experimental animal administration's supervisory and application system (FOTS-18/106800-1; ID 15469 and 23935).

Conflict of Interest The authors declare that they have no conflict of interest.

Open Access This article is licensed under a Creative Commons Attribution 4.0 International License, which permits use, sharing, adaptation, distribution and reproduction in any medium or format, as long as you give appropriate credit to the original author(s) and the source, provide a link to the Creative Commons licence, and indicate if changes were made. The images or other third party material in this article are included in the article's Creative Commons licence, unless indicated otherwise in a credit line to the material. If material is not included in the article's Creative Commons licence and your intended use is not permitted by statutory regulation or exceeds the permitted use, you will need to obtain permission directly from the copyright holder. To view a copy of this licence, visit http://creativecommons.org/licenses/by/4.0/.

\section{References}

1. Ripke S, Neale BM, Corvin A et al (2014) Biological insights from 108 schizophrenia-associated genetic loci. Nature 511:421427. https://doi.org/10.1038/nature13595

2. Harrison PJ (2015) Recent genetic findings in schizophrenia and their therapeutic relevance. J Psychopharmacol 29:85-96. https:// doi.org/10.1177/0269881114553647

3. Hawi Z, Tong J, Dark C, Yates H, Johnson B, Bellgrove MA (2018) The role of cadherin genes in five major psychiatric disorders: a literature update. Am J Med Genet 177:168-180. https:// doi.org/10.1002/ajmg.b.32592

4. Maurano MT, Humbert R, Rynes E, Thurman RE, Haugen E, Wang H, Reynolds AP, Sandstrom R et al (2012) Systematic localization of common disease-associated variation in regulatory DNA. Science 337:1190-1195. https://doi.org/10.1126/science. 1222794

5. Gershon ES, Grennan K, Busnello J, Badner JA, Ovsiew F, Memon S, Alliey-Rodriguez N, Cooper J et al (2014) A rare mutation of CACNA1C in a patient with bipolar disorder, and decreased gene expression associated with a bipolar-associated common SNP of CACNA1C in brain. Mol Psychiatry 19:890 894. https://doi.org/10.1038/mp.2013.107

6. Bigos KL, Mattay VS, Callicott JH, Straub RE, Vakkalanka R, Kolachana B, Hyde TM, Lipska BK et al (2010) Genetic variation in CACNA1C affects brain circuitries related to mental illness. Arch Gen Psychiatry 67:939-945. https://doi.org/10.1001/ archgenpsychiatry.2010.96 
7. Yoshimizu T, Pan JQ, Mungenast AE, Madison JM, Su S, Ketterman J, Ongur D, McPhie D et al (2015) Functional implications of a psychiatric risk variant within CACNA1C in induced human neurons. Mol Psychiatry 20:162-169. https://doi.org/10. 1038/mp.2014.143

8. Andrade A, Brennecke A, Mallat S, Brown J, Gomez-Rivadeneira J, Czepiel N, Londrigan L (2019) Genetic associations between voltage-gated calcium channels and psychiatric disorders. Int J Mol Sci:20. https://doi.org/10.3390/ijms20143537

9. Chan CS, Guzman JN, Ilijic E, Mercer JN, Rick C, Tkatch T, Meredith GE, Surmeier DJ (2007) "Rejuvenation" protects neurons in mouse models of Parkinson's disease. Nature 447:10811086. https://doi.org/10.1038/nature05865

10. Puopolo M, Raviola E, Bean BP (2007) Roles of subthreshold calcium current and sodium current in spontaneous firing of mouse midbrain dopamine neurons. J Neurosci 27:645-656. https://doi.org/10.1523/JNEUROSCI.4341-06.2007

11. Zamponi GW, Striessnig J, Koschak A, Dolphin AC (2015) The physiology, pathology, and pharmacology of voltage-gated calcium channels and their future therapeutic potential. Pharmacol Rev 67:821-870. https://doi.org/10.1124/pr.114.009654

12. Oguro-Okano M, Griesmann GE, Wieben ED et al (1992) Molecular diversity of neuronal-type calcium channels identified in small cell lung carcinoma. Mayo Clin Proc 67:1150-1159. https://doi.org/10.1016/S0025-6196(12)61144-6

13. Cross-Disorder Group of the Psychiatric Genomics Consortium (2013) Identification of risk loci with shared effects on five major psychiatric disorders: a genome-wide analysis. Lancet 381:13711379. https://doi.org/10.1016/S0140-6736(12)62129-1

14. Kabir ZD, Martínez-Rivera A, Rajadhyaksha AM (2017) From gene to behavior: L-type calcium channel mechanisms underlying neuropsychiatric symptoms. Neurotherapeutics 14:588-613. https://doi.org/10.1007/s13311-017-0532-0

15. Pinggera A, Striessnig J (2016) Cav 1.3 (CACNA1D) L-type $\mathrm{Ca} 2+$ channel dysfunction in CNS disorders. J Physiol Lond 594:5839-5849. https://doi.org/10.1113/JP270672

16. Hofer NT, Tuluc P, Ortner NJ, Nikonishyna YV, FernándesQuintero ML, Liedl KR, Flucher BE, Cox H et al (2020) Biophysical classification of a CACNA1D de novo mutation as a high-risk mutation for a severe neurodevelopmental disorder. Mol Autism 11. https://doi.org/10.1186/s13229-019-0310-4

17. Pinggera A, Lieb A, Benedetti B, Lampert M, Monteleone S, Liedl KR, Tuluc P, Striessnig J (2015) CACNA1D De novo mutations in autism spectrum disorders activate Cav1.3 L-type calcium channels. Biol Psychiatry 77:816-822. https://doi.org/10. 1016/j.biopsych.2014.11.020

18. Pinggera A, Mackenroth L, Rump A, Schallner J, Beleggia F, Wollnik B, Striessnig J (2017) New gain-of-function mutation shows CACNA1D as recurrently mutated gene in autism spectrum disorders and epilepsy. Hum Mol Genet 26:2923-2932. https://doi.org/10.1093/hmg/ddx175

19. Pinggera A, Negro G, Tuluc P, Brown MJ, Lieb A, Striessnig J (2018) Gating defects of disease-causing de novo mutations in Cav1.3 Ca2+ channels. Channels (Austin) 12:388-402. https:// doi.org/10.1080/19336950.2018.1546518

20. Scholl UI, Goh G, Stölting G, de Oliveira RC, Choi M, Overton JD, Fonseca AL, Korah R et al (2013) Somatic and germline CACNA1D calcium channel mutations in aldosterone-producing adenomas and primary aldosteronism. Nat Genet 45:1050-1054. https://doi.org/10.1038/ng.2695

21. Devor A, Andreassen O, Wang Y et al (2017) Genetic evidence for role of integration of fast and slow neurotransmission in schizophrenia. Mol Psychiatry 22:792-801. https://doi.org/10. 1038/mp.2017.33

22. Ross J, Gedvilaite E, Badner JA, Erdman C, Baird L, Matsunami N, Leppert M, Xing J et al (2016) A rare variant in CACNA1D segregates with 7 bipolar I disorder cases in a large pedigree. Mo Neuropsychiatry 2:145-150. https://doi.org/10.1159/000448041

23. Guan F, Li L, Qiao C, Chen G, Yan T, Li T, Zhang T, Liu X (2015) Evaluation of genetic susceptibility of common variants in CACNA1D with schizophrenia in Han Chinese. Sci Rep 5:12935. https://doi.org/10.1038/srep12935

24. Platzer J, Engel J, Schrott-Fischer A, Stephan K, Bova S, Chen H, Zheng H, Striessnig J (2000) Congenital deafness and sinoatrial node dysfunction in mice lacking class D L-type Ca2+ channels. Cell 102:89-97. https://doi.org/10.1016/s0092-8674(00)00013-1

25. Busquet P, Khoi Nguyen N, Schmid E, Tanimoto N, Seeliger MW, Ben-Yosef T, Mizuno F, Akopian A et al (2010) CaV1.3 L-type $\mathrm{Ca} 2+$ channels modulate depression-like behaviour in mice independent of deaf phenotype. Int J Neuropsychopharmacol 13: 499-513. https://doi.org/10.1017/S1461145709990368

26. McKinney BC, Sze W, Lee B, Murphy GG (2009) Impaired longterm potentiation and enhanced neuronal excitability in the amygdala of CaV1.3 knockout mice. Neurobiol Learn Mem 92:519528. https://doi.org/10.1016/j.nlm.2009.06.012

27. Sidi S, Busch-Nentwich E, Friedrich R, Schoenberger U, Nicolson $\mathrm{T}$ (2004) Gemini encodes a Zebrafish L-type calcium channel that localizes at sensory hair cell ribbon synapses. J Neurosci 24:42134223. https://doi.org/10.1523/JNEUROSCI.0223-04.2004

28. http://www.ensemble.org Gene: cacna $1 \mathrm{da}$ (ENSDARG00000102773) - Orthologue alignment - Danio rerio - Ensembl genome browser 99. https:/www.ensembl.org/Danio rerio/Gene/Compara_Ortholog/Alignment? $\mathrm{db}=$ core; $\mathrm{g}=$ ENSDARG00000102773; ; 1=ENSG00000157388;hom_id= 449865216; $\mathrm{r}=11: 36793473-36963988$. Accessed 23 Apr $20 \overline{20}$

29. http://www.ensemble.org Gene: cacna $1 \mathrm{db}$ (ENSDARG00000075917) - Orthologue alignment - Danio rerio - Ensembl genome browser 99. https://www.ensembl.org/Danio rerio/Gene/Compara_Ortholog/Alignment? $\mathrm{db}=$ core; $\mathrm{g}=$ ENSDARG00000075917;g1=ENSG00000142609; hom_id= 560456301;r=8:53204017-53260670. Accessed 23 Apr 2020

30. Nicolson T (2015) Ribbon synapses in zebrafish hair cells. Hear Res 330:170-177. https://doi.org/10.1016/j.heares.2015.04.003

31. Sheets L, Trapani JG, Mo W, Obholzer N, Nicolson T (2011) Ribeye is required for presynaptic CaV1.3a channel localization and afferent innervation of sensory hair cells. Development 138: 1309-1319. https://doi.org/10.1242/dev.059451

32. Sheets L, Kindt KS, Nicolson T (2012) Presynaptic CaV1.3 channels regulate synaptic ribbon size and are required for synaptic maintenance in sensory hair cells. J Neurosci 32:17273-17286. https://doi.org/10.1523/JNEUROSCI.3005-12.2012

33. Granato M, van Eeden FJ, Schach U, Trowe T, Brand M, Furutani-Seiki M, Haffter P, Hammerschmidt M et al (1996) Genes controlling and mediating locomotion behavior of the zebrafish embryo and larva. Development 123:399-413

34. Nicolson T, Rüsch A, Friedrich RW, Granato M, Ruppersberg JP, Nüsslein-Volhard C (1998) Genetic analysis of vertebrate sensory hair cell mechanosensation: the zebrafish circler mutants. Neuron 20:271-283. https://doi.org/10.1016/S0896-6273(00)80455-9

35. Prieto MJ, Gutierrez HC, Arévalo RA, Chiaramoni NS, Alonso SV (2012) Effect of risperidone and fluoxetine on the movement and neurochemical changes of zebrafish. OJMC 02:129-138. https://doi.org/10.4236/ojmc.2012.24016

36. Irons TD, Kelly P, Hunter DL et al (2013) Acute administration of dopaminergic drugs has differential effects on locomotion in larval zebrafish. Pharmacol Biochem Behav 103:792-813. https://doi. org/10.1016/j.pbb.2012.12.010

37. Tremolizzo L, Doueiri M-S, Dong E, Grayson DR, Davis J, Pinna G, Tueting P, Rodriguez-Menendez V et al (2005) Valproate corrects the schizophrenia-like epigenetic behavioral modifications induced by methionine in mice. Biol Psychiatry 57:500-509. https://doi.org/10.1016/j.biopsych.2004.11.046 
38. White HS, Alex AB, Pollock A, Hen N, Shekh-Ahmad T, Wilcox KS, McDonough JH, Stables JP et al (2012) A new derivative of valproic acid amide possesses a broad-spectrum antiseizure profile and unique activity against status epilepticus and organophosphate neuronal damage. Epilepsia 53:134-146. https://doi.org/10.1111/ j.1528-1167.2011.03338.x

39. Sanger Institute Zebrafish Mutation Project (2013) ZFIN Feature: sa17298. https://zfin.org/ZDB-ALT-131217-13434. Accessed 23 Apr 2020

40. Aleström P, D’Angelo L, Midtlyng PJ et al (2019) Zebrafish: housing and husbandry recommendations: laboratory animals. https://doi.org/10.1177/0023677219869037

41. Gawel K, Turski WA, van der Ent W, Mathai BJ, KirsteinSmardzewska KJ, Simonsen A, Esguerra CV (2020) Phenotypic characterization of larval zebrafish (Danio rerio) with partial knockdown of the cacnala gene. Mol Neurobiol 57:1904-1916. https://doi.org/10.1007/s12035-019-01860-x

42. Schnörr S, Steenbergen P, Richardson M, Champagne D (2012) Measuring thigmotaxis in larval zebrafish - ScienceDirect. https:// www.sciencedirect.com/science/article/pii/S0166432811008758? via\%3Dihub. Accessed 5 Dec 2019

43. Norton WHJ (2012) Measuring larval zebrafish behavior: locomotion, thigmotaxis, and startle. In: Kalueff AV, Stewart AM (eds) Zebrafish protocols for neurobehavioral research. Humana Press, Totowa, NJ, pp. 3-20

44. Banono NS, Esguerra CV (2020) Pharmacological validation of the prepulse inhibition of startle response in larval zebrafish using a commercial automated system and software. JoVE:61423. https://doi.org/10.3791/61423

45. Afrikanova T, Serruys A-SK, Buenafe OEM, Clinckers R, Smolders I, de Witte PAM, Crawford AD, Esguerra CV (2013) Validation of the zebrafish pentylenetetrazol seizure model: locomotor versus electrographic responses to antiepileptic drugs. PLoS One 8:e54166. https://doi.org/10.1371/journal.pone. 0054166

46. Aranda PS, LaJoie DM, Jorcyk CL (2012) Bleach gel: a simple agarose gel for analyzing RNA quality. ELECTROPHORESIS 33:366-369. https://doi.org/10.1002/elps.201100335

47. Basnet RM, Zizioli D, Taweedet S, Finazzi D, Memo M (2019) Zebrafish larvae as a behavioral model in neuropharmacology. Biomedicines 7:23. https://doi.org/10.3390/biomedicines7010023

48. Best JD, Berghmans S, Hunt JJFG, Clarke SC, Fleming A, Goldsmith P, Roach AG (2008) Non-associative learning in larval zebrafish. Neuropsychopharmacology 33:1206-1215. https://doi. org/10.1038/sj.npp.1301489

49. Baraban SC, Dinday MT, Hortopan GA (2013) Drug screening in Scn1 a zebrafish mutant identifies clemizole as a potential Dravet syndrome treatment. Nat Commun 4:2410. https://doi.org/10. $1038 /$ ncomms 3410

50. Gawel K, Kukula-Koch W, Nieoczym D, Stepnik K, van der Ent W, Banono NS, Tarabasz D, Turski WA et al (2020) The influence of palmatine isolated from Berberis sibirica radix on pentylenetetrazole-induced seizures in zebrafish. Cells 9:1233. https://doi.org/10.3390/cells9051233

51. Kalueff AV, Gebhardt M, Stewart AM, Cachat JM, Brimmer M, Chawla JS, Craddock C, Kyzar EJ et al (2013) Towards a comprehensive catalog of zebrafish behavior 1.0 and beyond. Zebrafish 10:70-86. https://doi.org/10.1089/zeb.2012.0861

52. Tiraboschi E, Martina S, van der Ent W et al (2020) New insights into the early mechanisms of epileptogenesis in a zebrafish model of Dravet syndrome. Epilepsia 61:549-560. https://doi.org/10. 1111/epi.16456

53. Maximino C, de Brito TM, da Silva Batista AW, Herculano AM, Morato S, Gouveia A Jr (2010) Measuring anxiety in zebrafish: A critical review. Behav Brain Res 214:157-171. https://doi.org/10. 1016/j.bbr.2010.05.031
54. Seibt KJ, da Luz Oliveira R, Zimmermann FF et al (2010) Antipsychotic drugs prevent the motor hyperactivity induced by psychotomimetic MK-801 in zebrafish (Danio rerio). Behav Brain Res 214:417-422. https://doi.org/10.1016/j.bbr.2010.06.014

55. Seibt KJ, Piato AL, da Luz OR et al (2011) Antipsychotic drugs reverse MK-801-induced cognitive and social interaction deficits in zebrafish (Danio rerio). Behav Brain Res 224:135-139. https:// doi.org/10.1016/j.bbr.2011.05.034

56. Kedra M, Banasiak K, Kisielewska K, Wolinska-Niziol L, Jaworski J, Zmorzynska J (2020) TrkB hyperactivity contributes to brain dysconnectivity, epileptogenesis, and anxiety in zebrafish model of tuberous sclerosis complex. Proc Natl Acad Sci U S A 117:2170-2179. https://doi.org/10.1073/pnas.1910834117

57. Scheldeman C, Mills JD, Siekierska A, Serra I, Copmans D, Iyer AM, Whalley BJ, Maes J et al (2017) mTOR-related neuropathology in mutant tsc2 zebrafish: Phenotypic, transcriptomic and pharmacological analysis. Neurobiol Dis 108:225-237. https://doi.org/ 10.1016/j.nbd.2017.09.004

58. Li F, Lin J, Liu X et al (2018) Characterization of the locomotor activities of zebrafish larvae under the influence of various neuroactive drugs. Ann Transl Med 6:173. https://doi.org/10.21037/ atm.2018.04.25

59. Liu X, Zhang Y, Lin J, et al (2016) Frontiers | social preference deficits in juvenile zebrafish induced by early chronic exposure to sodium valproate | Behavioral Neuroscience. https://www. frontiersin.org/articles/10.3389/fnbeh.2016.00201/full. Accessed 5 Dec 2019

60. Lundegaard PR, Anastasaki C, Grant NJ, Sillito RR, Zich J, Zeng Z, Paranthaman K, Larsen AP et al (2015) MEK inhibitors reverse cAMP-mediated anxiety in zebrafish. Chem Biol 22:1335-1346. https://doi.org/10.1016/j.chembiol.2015.08.010

61. Zhang Y, Kecskés A, Copmans D, Langlois M, Crawford AD, Ceulemans B, Lagae L, de Witte PAM et al (2015) Pharmacological characterization of an antisense knockdown zebrafish model of Dravet syndrome: inhibition of epileptic seizures by the serotonin agonist fenfluramine. PLoS One 10: e0125898. https://doi.org/10.1371/journal.pone.0125898

62. Pena IA, Roussel Y, Daniel K et al (2017) Pyridoxine-dependent epilepsy in zebrafish caused by Aldh7a1 deficiency. Genetics genetics.300137.2017. https://doi.org/10.1534/genetics.117.300137

63. Klassen T, Davis C, Goldman A, Burgess D, Chen T, Wheeler D, McPherson J, Bourquin T et al (2011) Exome sequencing of ion channel genes reveals complex profiles confounding personal risk assessment in epilepsy. Cell 145:1036-1048. https://doi.org/10. 1016/j.cell.2011.05.025

64. Jones C, Watson D, Fone K (2011) Animal models of schizophrenia. Br J Pharmacol 164:1162-1194. https://doi.org/10.1111/j. 1476-5381.2011.01386.x

65. Michelotti P, Quadros VA, Pereira ME, Rosemberg DB (2018) Ketamine modulates aggressive behavior in adult zebrafish. Neurosci Lett 684:164-168. https://doi.org/10.1016/j.neulet. 2018.08.009

66. De Campos EG, Bruni AT, De Martinis BS (2015) Ketamine induces anxiolytic effects in adult zebrafish: a multivariate statistics approach. Behav Brain Res 292:537-546. https://doi.org/10. 1016/j.bbr.2015.07.017

67. Chen J, Patel R, Friedman TC, Jones KS (2010) The behavioral and pharmacological actions of NMDA receptor antagonism are conserved in zebrafish larvae. Int J Comp Psychol 23:82-90

68. Kyzar E, Stewart AM, Landsman S, Collins C, Gebhardt M, Robinson K, Kalueff AV (2013) Behavioral effects of bidirectional modulators of brain monoamines reserpine and d-amphetamine in zebrafish. Brain Res 1527:108-116. https://doi.org/10.1016/j. brainres.2013.06.033

69. Irons TD, MacPhail RC, Hunter DL, Padilla S (2010) Acute neuroactive drug exposures alter locomotor activity in larval 
zebrafish. Neurotoxicol Teratol 32:84-90. https://doi.org/10. 1016/j.ntt.2009.04.066

70. Lautar SL, Rojas C, Slusher BS, Wozniak KM, Wu Y, Thomas AG, Waldon D, Li W et al (2005) DPP IV inhibitor blocks mescaline-induced scratching and amphetamine-induced hyperactivity in mice. Brain Res 1048:177-184. https://doi.org/10.1016/j. brainres.2005.04.069

71. Alttoa A, Eller M, Herm L, Rinken A, Harro J (2007) Amphetamine-induced locomotion, behavioral sensitization to amphetamine, and striatal D2 receptor function in rats with high or low spontaneous exploratory activity: Differences in the role of locus coeruleus. Brain Res 1131:138-148. https://doi.org/10. 1016/j.brainres.2006.10.075

72. Gormley S, Rompré P-P (2011) Blockade of mGLUR5 receptors differentially alters amphetamine-induced enhancement of locomotor activity and of brain stimulation reward. J Psychopharmacol 25:393-401. https://doi.org/10.1177/ 0269881110367460

73. Saylor AJ, McGinty JF (2008) Amphetamine-induced locomotion and gene expression are altered in BDNF heterozygous mice. Genes Brain Behav 7:906-914. https://doi.org/10.1111/j.1601183X.2008.00430.x

74. Salahpour A, Ramsey AJ, Medvedev IO, Kile B, Sotnikova TD, Holmstrand E, Ghisi V, Nicholls PJ et al (2008) Increased amphetamine-induced hyperactivity and reward in mice overexpressing the dopamine transporter. Proc Natl Acad Sci 105:44054410. https://doi.org/10.1073/pnas.0707646105

75. Pletnikov MV, Ayhan Y, Nikolskaia O, Xu Y, Ovanesov MV, Huang H, Mori S, Moran TH et al (2008) Inducible expression of mutant human DISC1 in mice is associated with brain and behavioral abnormalities reminiscent of schizophrenia. Mol Psychiatry 13:173-186. https://doi.org/10.1038/sj.mp.4002079

76. Karl T, Duffy L, Scimone A, Harvey RP, Schofield PR (2007) Altered motor activity, exploration and anxiety in heterozygous neuregulin 1 mutant mice: Implications for understanding schizophrenia. Genes Brain Behav 6:677-687. https://doi.org/10.1111/j. 1601-183X.2006.00298.X

77. Thyme SB, Pieper LM, Li EH et al (2019) Phenotypic landscape of schizophrenia-associated genes defines candidates and their shared functions. Cell 177:478-491.e20. https://doi.org/10.1016/ j.cell.2019.01.048

78. Wolman MA, Jain RA, Liss L, Granato M (2011) Chemical modulation of memory formation in larval zebrafish. Proc Natl Acad Sci 108:15468-15473. https://doi.org/10.1073/pnas.1107156108

79. Randlett O, Haesemeyer M, Forkin G et al (2019) Distributed plasticity drives visual habituation learning in larval Zebrafish. Curr Biol 29:1337-1345.e4. https://doi.org/10.1016/j.cub.2019. 02.039

80. Braff DL (1992) Gating and habituation of the startle reflex in schizophrenic patients. Arch Gen Psychiatry 49:206-215. https:// doi.org/10.1001/archpsyc.1992.01820030038005

81. Avery SN, McHugo M, Armstrong K, Blackford JU, Woodward ND, Heckers S (2019) Disrupted habituation in the early stage of psychosis. Biological Psychiatry: Cognitive Neuroscience and Neuroimaging 4:1004-1012. https://doi.org/10.1016/j.bpsc.2019. 06.007

82. Aggernaes B, Glenthoj BY, Ebdrup BH, Rasmussen H, Lublin H, Oranje B (2010) Sensorimotor gating and habituation in antipsychotic-naive, first-episode schizophrenia patients before and after 6 months' treatment with quetiapine. Int J Neuropsychopharmacol 13:1383-1395. https://doi.org/10.1017/S1461145710000787

83. Quednow BB, Frommann I, Berning J, Kühn KU, Maier W, Wagner M (2008) Impaired sensorimotor gating of the acoustic startle response in the prodrome of schizophrenia. Biol Psychiatry 64:766-773. https://doi.org/10.1016/j.biopsych.2008.04.019
84. Quednow BB, Wagner M, Westheide J, Beckmann K, Bliesener N, Maier W, Kühn KU (2006) Sensorimotor gating and habituation of the startle response in schizophrenic patients randomly treated with amisulpride or olanzapine. Biol Psychiatry 59:536545. https://doi.org/10.1016/j.biopsych.2005.07.012

85. Cadenhead KS, Swerdlow NR, Shafer KM, Diaz M, Braff DL (2000) Modulation of the startle response and startle laterality in relatives of schizophrenic patients and in subjects with schizotypal personality disorder: evidence of inhibitory deficits. Am J Psychiatry 157:1660-1668. https://doi.org/10.1176/appi.ajp.157. 10.1660

86. Duncan GE, Moy SS, Lieberman JA, Koller BH (2006) Effects of haloperidol, clozapine, and quetiapine on sensorimotor gating in a genetic model of reduced NMDA receptor function. Psychopharmacology 184:190-200. https://doi.org/10.1007/ s00213-005-0214-1

87. Kanahara N, Shimizu E, Ohgake S, Fujita Y, Kohno M, Hashimoto T, Matsuzawa D, Shirayama Y et al (2008) Glycine and d-serine, but not d-cycloserine, attenuate prepulse inhibition deficits induced by NMDA receptor antagonist MK-801. Psychopharmacology 198:363-374. https://doi.org/10.1007/ s00213-008-1151-6

88. Papaleo F, Yang F, Garcia S, Chen J, Lu B, Crawley JN, Weinberger DR (2012) Dysbindin-1 modulates prefrontal cortical activity and schizophrenia-like behaviors via dopamine/D2 pathways. Mol Psychiatry 17:85-98. https://doi.org/10.1038/mp. 2010.106

89. Baig SM, Koschak A, Lieb A, Gebhart M, Dafinger C, Nürnberg G, Ali A, Ahmad I et al (2011) Loss of Cav1.3 (CACNA1D) function in a human channelopathy with bradycardia and congenital deafness. Nat Neurosci 14:77-84. https://doi.org/10.1038/nn. 2694

90. Liaqat K, Raza SI, Lee K et al (2019) Identification of CACNA1D variants associated with sinoatrial node dysfunction and deafness in additional Pakistani families reveals a clinical significance. J Hum Genet 64:153-160. https://doi.org/10.1038/s10038-0180542-8

91. Giacomini NJ, Rose B, Kobayashi K, Guo S (2006) Antipsychotics produce locomotor impairment in larval zebrafish. Neurotoxicol Teratol 28:245-250. https://doi.org/10. 1016/j.ntt.2006.01.013

92. Idalencio R, Kalichak F, Rosa JGS, Oliveira TA, Koakoski G, Gusso D, Abreu MS, Giacomini ACV et al (2015) Waterborne Risperidone decreases stress response in zebrafish. PLoS One 10: e0140800. https://doi.org/10.1371/journal.pone.0140800

93. Magno LDP, Fontes A, Gonçalves BMN, Gouveia A (2015) Pharmacological study of the light/dark preference test in zebrafish (Danio rerio): waterborne administration. Pharmacol Biochem Behav 135:169-176. https://doi.org/10.1016/j.pbb. 2015.05.014

94. Bruni G, Rennekamp AJ, Velenich A, McCarroll M, Gendelev L, Fertsch E, Taylor J, Lakhani P et al (2016) Zebrafish behavioral profiling identifies multitarget antipsychotic-like compounds. Nat Chem Biol 12:559-566. https://doi.org/10.1038/nchembio.2097

95. Boehmler W, Carr T, Thisse C, Thisse B, Canfield VA, Levenson $\mathrm{R}$ (2007) D4 dopamine receptor genes of zebrafish and effects of the antipsychotic clozapine on larval swimming behaviour. Genes Brain Behav 6:155-166. https://doi.org/10.1111/j.1601-183X. 2006.00243.x

96. Ghodke-Puranik Y, Thorn CF, Lamba JK, Leeder JS, Song W, Birnbaum AK, Altman RB, Klein TE (2013) Valproic acid pathway: pharmacokinetics and pharmacodynamics. Pharmacogenet Genomics 23:236-241. https://doi.org/10.1097/FPC. 0b013e32835ea0b2

97. Johannesen KM, Gardella E, Linnankivi T, Courage C, de Saint Martin A, Lehesjoki AE, Mignot C, Afenjar A et al (2018) 
Defining the phenotypic spectrum of SLC6A1 mutations. Epilepsia 59:389-402. https://doi.org/10.1111/epi.13986

98. Franceschetti S, Hamon B, Heinemann U (1986) The action of valproate on spontaneous epileptiform activity in the absence of synaptic transmission and on evoked changes in $[\mathrm{Ca} 2+] 0$ and $[\mathrm{K}+$ ]0 in the hippocampal slice. Brain Res 386:1-11. https://doi.org/ 10.1016/0006-8993(86)90135-6

99. Leysen JE, Janssen PMF, Schotte A, Luyten WHML, Megens AAHP (1993) Interaction of antipsychotic drugs with neurotransmitter receptor sites in vitro and in vivo in relation to pharmacological and clinical effects: role of 5HT2 receptors Psychopharmacology 112:S40-S54. https://doi.org/10.1007/ BF02245006

100. Kalichak F, Idalencio R, da Rosa JGS, Barcellos HHA, Fagundes M, Piato A, Barcellos LJG (2017) Psychotropic in the environment: risperidone residues affect the behavior of fish larvae. Sci Rep 7:14121. https://doi.org/10.1038/s41598-017-14575-7

101. Uhlen M, Fagerberg L, Hallstrom BM, Lindskog C, Oksvold P, Mardinoglu A, Sivertsson A, Kampf C et al (2015) Tissue-based map of the human proteome. Science 347:1260419-1260419. https://doi.org/10.1126/science.1260419

102. Tissue expression of CACNA1D - Summary - The HUMAN PROTEIN ATLAS. https://www.proteinatlas.org/ ENSG00000157388-CACNA1D/tissue. Accessed 25 Jun 2020

103. Hetzenauer A, Sinnegger-Brauns MJ, Striessnig J, Singewald N (2006) Brain activation pattern induced by stimulation of L-type $\mathrm{Ca} 2+-$ channels: contribution of $\mathrm{CaV} 1.3$ and $\mathrm{CaV} 1.2$ isoforms. Neuroscience 139:1005-1015. https://doi.org/10.1016/j. neuroscience.2006.01.059

104. Gene Detail: Allen brain atlas: mouse brain. http://mouse.brainmap.org/gene/show/12074. Accessed 25 Jun 2020

105. Lein ES, Hawrylycz MJ, Ao N, Ayres M, Bensinger A, Bernard A, Boe AF, Boguski MS et al (2007) Genome-wide atlas of gene expression in the adult mouse brain. Nature 445:168-176. https:// doi.org/10.1038/nature05453
106. Gawel K, Banono NS, Michalak A, Esguerra CV (2019) A critical review of zebrafish schizophrenia models: time for validation? Neurosci Biobehav Rev 107:6-22. https://doi.org/10.1016/j. neubiorev.2019.08.001

107. Gawel K, Langlois M, Martins T, van der Ent W, Tiraboschi E, Jacmin M, Crawford AD, Esguerra CV (2020) Seizing the moment: zebrafish epilepsy models. Neurosci Biobehav Rev 116:120. https://doi.org/10.1016/j.neubiorev.2020.06.010

108. Eimon PM, Ghannad-Rezaie M, De Rienzo G et al (2018) Brain activity patterns in high-throughput electrophysiology screen predict both drug efficacies and side effects. Nat Commun 9:219. https://doi.org/10.1038/s41467-017-02404-4

109. Suster ML, Abe G, Schouw A, Kawakami K (2011) Transposonmediated BAC transgenesis in zebrafish. Nat Protoc 6:1998-2021. https://doi.org/10.1038/nprot.2011.416

110. Kawakami K (2005) Transposon tools and methods in zebrafish. Dev Dyn 234:244-254. https://doi.org/10.1002/dvdy.20516

111. Hwang WY, Fu Y, Reyon D, Gonzales APW, Joung JK, Yeh JRJ (2015) Targeted mutagenesis in zebrafish using CRISPR RNAguided nucleases. In: Lundgren M, Charpentier E, Fineran PC (eds) CRISPR. Springer New York, New York, NY, pp. 317-334

112. Arranz MJ, Rivera M, Munro JC (2011) Pharmacogenetics of response to antipsychotics in patients with schizophrenia. CNS Drugs 25:933-969. https://doi.org/10.2165/11595380000000000-00000

113. Vita A, Minelli A, Barlati S, Deste G, Giacopuzzi E, Valsecchi P, Turrina C, Gennarelli M (2019) Treatment-resistant schizophrenia: genetic and neuroimaging correlates. Front Pharmacol 10: 402. https://doi.org/10.3389/fphar.2019.00402

Publisher's Note Springer Nature remains neutral with regard to jurisdictional claims in published maps and institutional affiliations. 
Supplementary Materials

Table 1: List of primers for rt- and q-PCR

\begin{tabular}{|l|l|l|l|}
\hline Target name & $\begin{array}{l}\text { Gene } \\
\text { symbol }\end{array}$ & Forward primer & Reverse primer \\
\hline $\begin{array}{l}\text { glyceraldehyde-3- } \\
\text { phosphate } \\
\text { dehydrogenase }\end{array}$ & gapdh & 5' GTGGAGTCTACTGGTGTCTTC 3' $^{\prime}$ & 5' GTGCAGGAGGCATTGCTTACA 3' $^{\prime}$ \\
\hline $\begin{array}{l}\text { ribosomal protein S18 } \\
\text { both 201 and 202 } \\
\text { transcript variants) }\end{array}$ & rps18 & 5' AGTTCTCCAGCCCTCTTATT 3' $^{\prime}$ & 5' TCAACACGAACATTGATGGA 3' $^{\prime}$ \\
\hline $\begin{array}{l}\text { cacnalda (transcript } \\
\text { variant 202) }\end{array}$ & cacnalda & 5' GGCTATGCAGATTATGTCTTCAC 3' & 5' TGGACGCAAAACTCTCAGAA 3' \\
\hline $\begin{array}{l}\text { cacnalda (transcript } \\
\text { variant 201) }\end{array}$ & cacnalda & 5'TACTTGGTTACTTTGACTATGCTTTCA3' & 5'CTTTAGTCCTTTAGCTCTGTTTATGG3' \\
\hline
\end{tabular}

Table 2: Effects of neuroactive drugs on larval behaviour in the light-dark assay: Twoway ANOVA test. HALO- haloperidol, RISP- risperidone, VPA- valproic acid.

\begin{tabular}{|l|l|l|l|l|}
\hline Treatment & Exposure time & ANOVA table & $\mathrm{F}(\mathrm{DFn}, \mathrm{DFd})$ & $\mathrm{P}$ value \\
\hline $5 \mu \mathrm{M}$ RISP & \multirow{2}{*}{$\mathrm{h} \mathrm{h}$} & Interaction & $\mathrm{F}(1,60)=0.2889$ & $\mathrm{P}=0.5929$ \\
\cline { 3 - 5 } & & Illumination & $\mathrm{F}(1,60)=68.10$ & $\mathrm{P}<0.0001$ \\
\cline { 3 - 5 } & & Genotype & $\mathrm{F}(1,60)=2.294$ & $\mathrm{P}=0.1352$ \\
\hline $5 \mu \mathrm{M}$ RISP & \multirow{2}{*}{$24 \mathrm{~h}$} & Interaction & $\mathrm{F}(1,94)=0.03859$ & $\mathrm{P}=0.8447$ \\
\cline { 3 - 5 } & & Illumination & $\mathrm{F}(1,94)=117.8$ & $\mathrm{P}<0.0001$ \\
\cline { 3 - 5 } & & Genotype & $\mathrm{F}(1,94)=2.125$ & $\mathrm{P}=0.1483$ \\
\hline $50 \mu \mathrm{M}$ HALO & $2 \mathrm{~h}$ & Interaction & $\mathrm{F}(1,78)=0.2112$ & $\mathrm{P}=0.6471$ \\
\cline { 3 - 5 } & & Illumination & $\mathrm{F}(1,78)=0.01388$ & $\mathrm{P}=0.9065$ \\
\hline & & Genotype & $\mathrm{F}(1,78)=8.121$ & $\mathrm{P}=0.0056$ \\
\hline $100 \mu \mathrm{M}$ VPA & $2 \mathrm{~h}$ & Interaction & $\mathrm{F}(1,58)=1.046$ & $\mathrm{P}=0.3107$ \\
\cline { 3 - 5 } & & Illumination & $\mathrm{F}(1,58)=201.8$ & $\mathrm{P}<0.0001$ \\
\cline { 3 - 5 } & & Genotype & $\mathrm{F}(1,58)=0.1158$ & $\mathrm{P}=0.7349$ \\
\hline
\end{tabular}


Table 3: Effects of neuroactive drugs on larval behaviour in the startle response to dark flashes assay: Unpaired $t$-test. HALO- haloperidol, RISP- risperidone, VPA- valproic acid.

\begin{tabular}{|l|l|l|l|}
\hline \multirow{2}{*}{ Unpaired $t$-test } & \multirow{2}{*}{ Treatment } & \multicolumn{2}{|c|}{ P-value } \\
\cline { 3 - 4 } & $0.1 \%$ DMSO $2 \mathrm{~h}$ & 0.0293 & 0.0444 \\
\hline WT vs sa17298/WT & $5 \mu \mathrm{M}$ RISP 2 h & 0.2059 & 0.7701 \\
\hline WT vs sa17298/WT & $0.1 \%$ DMSO 24 h & 0.0462 & 0.0047 \\
\hline WT vs sa17298/WT & $5 \mu$ M RISP 24 h & 0.8743 & 0.002 \\
\hline WT vs sa17298/WT & $0.5 \%$ DMSO 2 h & 0.0345 & 0.4206 \\
\hline WT vs sa17298/WT & $50 \mu$ M HALO 2 h & 0.1992 & 0.9147 \\
\hline WT vs sa17298/WT & $0.5 \%$ DMSO 2 h & 0.0357 & 0.0115 \\
\hline WT vs sa17298/WT & $100 \mu$ M VPA 2 h & 0.3924 & 0.6849 \\
\hline WT vs sa17298/WT & & & \\
\hline
\end{tabular}


Fig.1

Representative alignment of cacnalda transcript variants 201(v201) and 202 (v202) showing the primer sequences used for rt- and q-PCR.

V201 CCAGTTCGAG TCGCC Cacnalda (v201 and v202) Fwd

V202 CCAGTTCGAG TCGCCTGCCA CAAGCTGATA AACACCACA TCTTCACCAA CCTCATCCTT GTGTTCATTA TGCTAAGCTC AGCGTCATTG GCTGCTGAGG

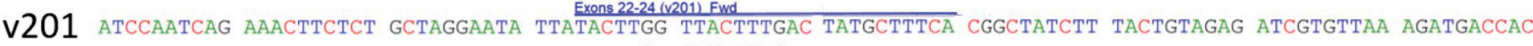

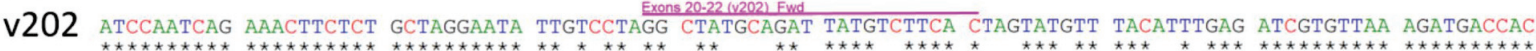

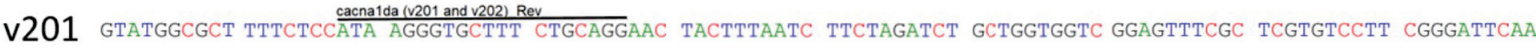

v202 GTATGGCGCT TTTCTCCATA AGGGTGCTTT CTGCAGGAAC TACTTTAATC TTCTAGATCT GCTGGTGGT GGAGTTTGC TCGTGTCTT CGGGATTCAA

V201 TCATCGGCCA TCTCAGTGGT GAAGATTCTG AGGGTTCTGA GAGTTTTGCG TCCACTCAGA Exons 22-24 (v201) Rev

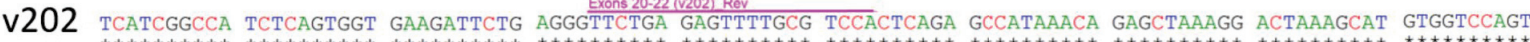

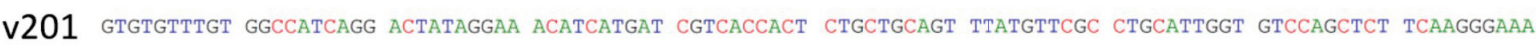

V202 GTGTGTTTGT GGCCATCAGG ACTATAGGAA ACATCATGAT CGTCACCACT CTGCTGCAGT TTATGTTCGC CTGCATTGGT GTCCAGCTCT TCAAGGGAAA 


\section{Fig. 2}

Observable dismorphologies in 5 dpf larvae when adult (male and female) heterozygous sa17298 (obtained directly from ZIRC) are in-crossed. Black arrow - craniofacial abnormalities, blue arrow - upward curved tail, yellow arrow - downward curved tail, purple arrow - pericardial edema, red arrow - small or fused eyes, hyperpigmentation and yolk sac necrosis. With some homozygotes looking indistinguishable from WT siblings. Scale bar (A-C): $1 \mathrm{~mm}$.

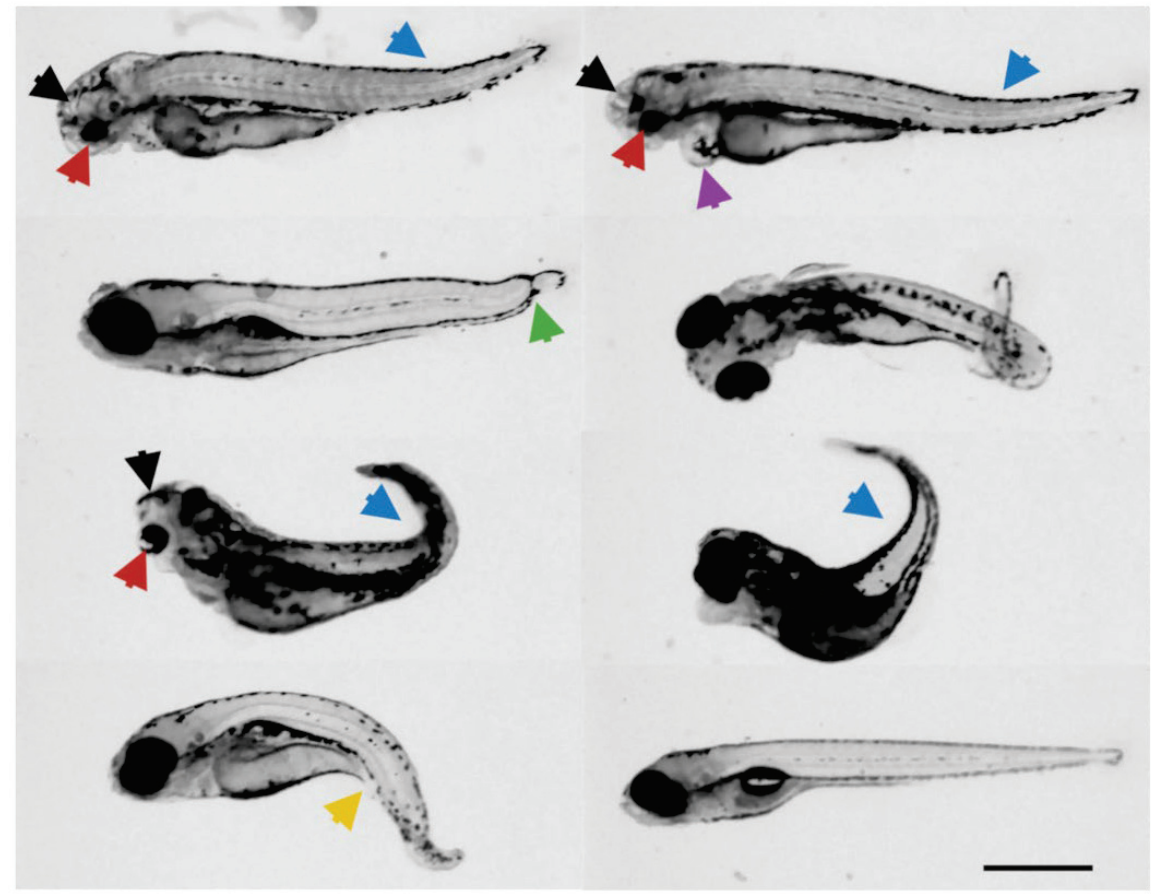


Fig. 3

Effects of neuroactive drugs on the behaviour of 6 dpf WT and heterozygous sa17298 larvae in the startle response to dark flashes test. Larvae were exposed to different neuroactive drugs. Each dot represents individual larval measurement. Data analyzed using one-way ANOVA followed by multiple $t$-test. Data represented as mean \pm SD. A) 2 h RISP, B) 24 h RISP, C) 2 h HALO, D) 2 h VPA. HALO- haloperidol, RISP- risperidone, VPA- valproic acid.

$* \mathrm{p}<0.05\left[\right.$ cacnalda ${ }^{W T / W T}$ vs cacnalda $a^{\text {sal7298/WT}]}$ in respective groups \# $\mathrm{p}<0.05$ [cacnalda ${ }^{W T / W T}$ vs cacnalda $\left.{ }^{W T / W T}\right]$ and [cacnalda ${ }^{\text {sal7298/WT }}$ vs cacnalda ${ }^{\text {sal7298/WT }}$ ]
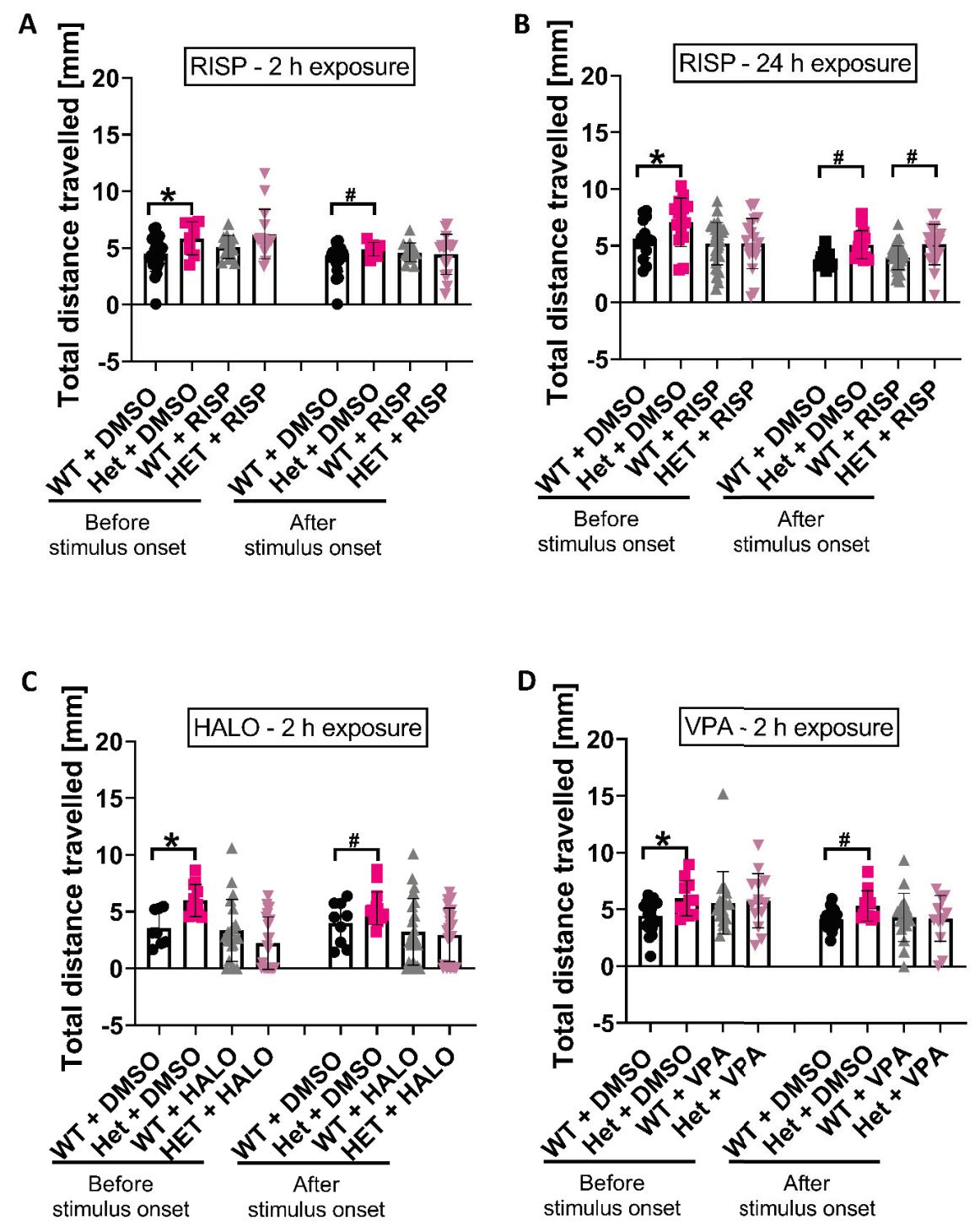
Fig. 4

Representative EEG recording showing the seizure-like discharges recorded from the optic tectum in zebrafish WT and sa17298/WT larvae at 6 dpf. A) Fragment of representative EEG recording from WT larvae. B) Fragment of recording from sa17298/WT larvae demonstrating the background and discharges.

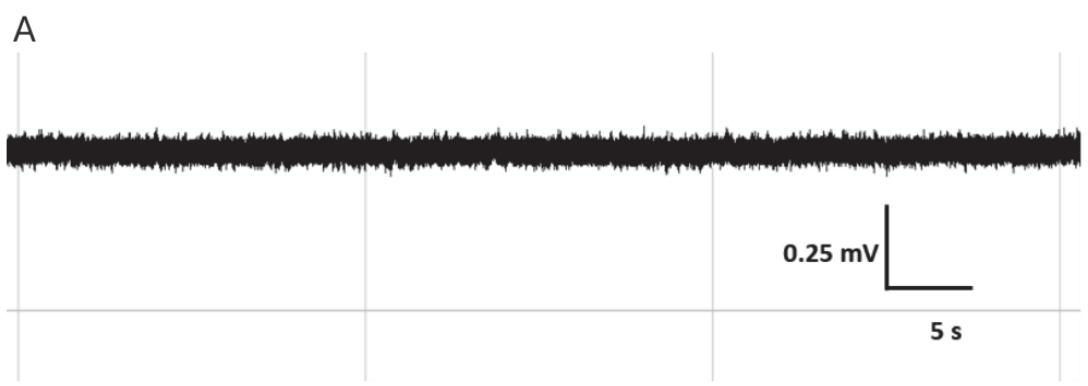

B

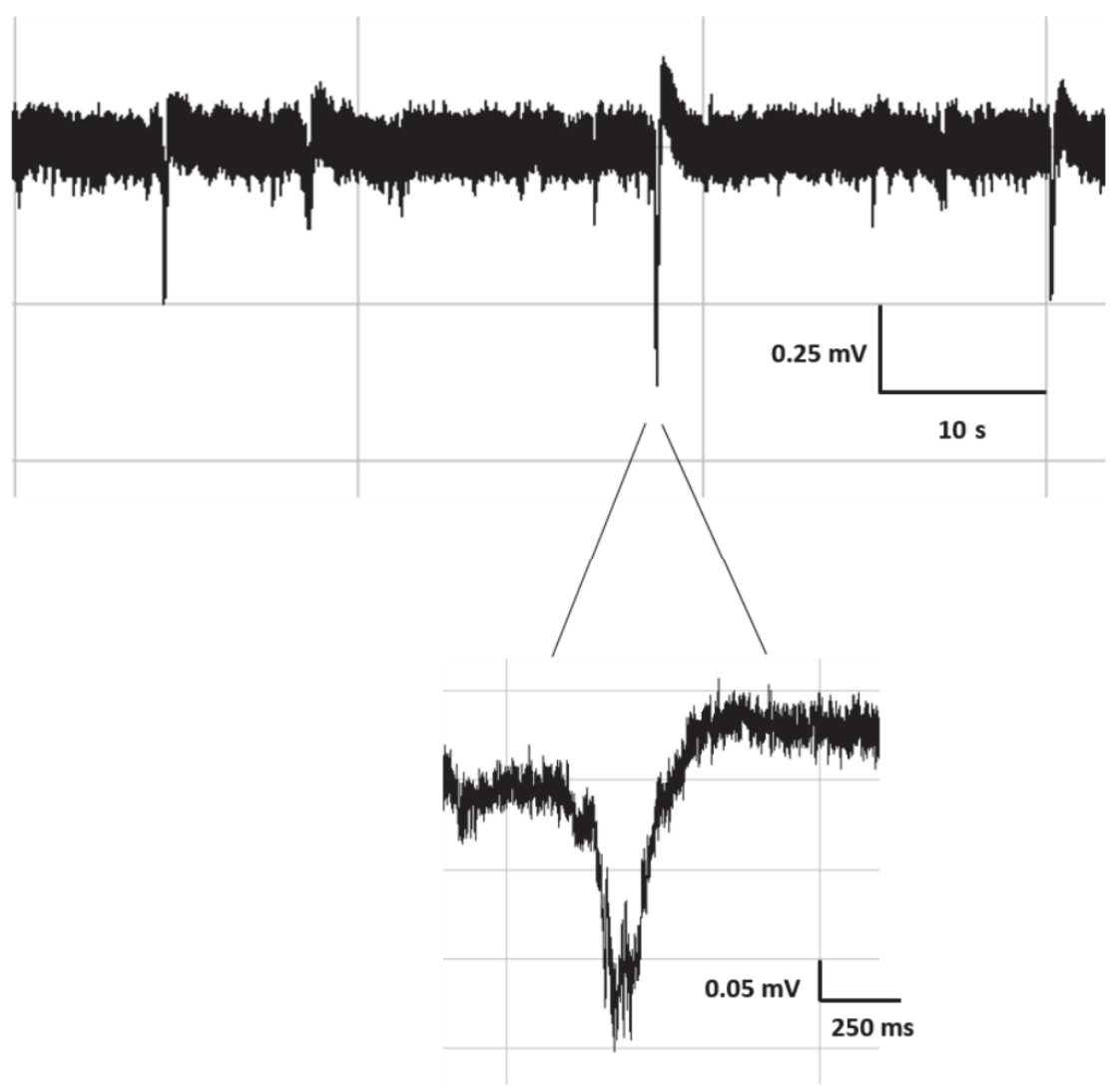

